\title{
A Meshless Approach to Solving Partial Differential Equations Using the Finite Cloud Method for the Purposes of Computer Aided Design
}

by

Daniel Rutherford Burke, B.Eng

\author{
A Thesis submitted to \\ the Faculty of Graduate and Post Doctoral Affairs \\ in partial fulfilment of \\ the requirements for the degree of \\ Doctor of Philosophy \\ Ottawa Carleton Institute for \\ Electrical and Computer Engineering
}

Department of Electronics

Carleton University

Ottawa, Ontario, Canada

January 2013 
Library and Archives

Canada

Published Heritage

Branch

395 Wellington Street

Ottawa ON K1A ON4

Canada
Bibliothèque et

Archives Canada

Direction du

Patrimoine de l'édition

395 , rue Wellington

Ottawa ON K1A ON4

Canada
Your file Votre référence

ISBN: 978-0-494-94524-7

Our file Notre référence

ISBN: $978-0-494-94524-7$
NOTICE:

The author has granted a nonexclusive license allowing Library and Archives Canada to reproduce, publish, archive, preserve, conserve, communicate to the public by telecommunication or on the Internet, loan, distrbute and sell theses worldwide, for commercial or noncommercial purposes, in microform, paper, electronic and/or any other formats.

The author retains copyright ownership and moral rights in this thesis. Neither the thesis nor substantial extracts from it may be printed or otherwise reproduced without the author's permission.
AVIS:

L'auteur a accordé une licence non exclusive permettant à la Bibliothèque et Archives Canada de reproduire, publier, archiver, sauvegarder, conserver, transmettre au public par télécommunication ou par l'Internet, prêter, distribuer et vendre des thèses partout dans le monde, à des fins commerciales ou autres, sur support microforme, papier, électronique et/ou autres formats.

L'auteur conserve la propriété du droit d'auteur et des droits moraux qui protege cette thèse. $\mathrm{Ni}$ la thèse ni des extraits substantiels de celle-ci ne doivent être imprimés ou autrement reproduits sans son autorisation.
In compliance with the Canadian Privacy Act some supporting forms may have been removed from this thesis.

While these forms may be included in the document page count, their removal does not represent any loss of content from the thesis.
Conformément à la loi canadienne sur la protection de la vie privée, quelques formulaires secondaires ont été enlevés de cette thèse.

Bien que ces formulaires aient inclus dans la pagination, il n'y aura aucun contenu manquant. 
Copyright (C)

2013 - Daniel Rutherford Burke, B.Eng 


\section{Abstract}

Modelling tools which are able to solve partial differential equations with increasing accuracy, complexity and ease of use are essential for engineers. Two main methods of solving these types of problems are the Finite Difference Method, and the Finite Element Method, both of which rely on a mesh to discretize the domain or solution space. These meshed methods are widely used and studied. However, they suffer from a variety of problems related to their construction and rigidity. A third type of solution, known as meshless or meshfree methods, are able to avoid meshing problems and are currently being heavily researched. In this thesis a promising type of meshless method, the Finite Cloud Method, is investigated and a ' $\mathrm{C}$ ' program implementing the method has been written. The method is applied to a range of problems including scalar and vectorial equations, coupled field and both time independent and time dependent solutions. In particular, the method is extended to include inhomogeneous domains (multiple materials) and spatially dependent material parameters. Physical situations addressed include: Heat Flow, Schrodinger's Equation, Maxwell's Equations and optical mode solving. Results for the various equation types have been very promising and in high agreement with both analytically and numerically solved solutions. As well, several improvements to the method have been developed and are detailed. The method is shown to be versatile, robust and highly accurate. 


\section{Acknowledgments}

I wish to thank, first and foremost, my supervisor prof.,Tom Smy, without whom this thesis would not have been possible. His patience and clarity when explaining concepts from the very simple to the overly complicated were invaluable to my research and progress throughout this work. He provided encouragement and insights when I was stuck on theoretical roadblocks, coding bugs or general thesis malaise, many times giving me the drive to continue on. His confidence in me gave me the confidence to pursue this degree and tackle every new challenge knowing that it could be surmounted.

I am indebted to the other professors and graduate students in the department with whom I spent far too many hours discussing our current stumbling blocks, solving the world's problems, or generally providing entertaining and often thought provoking distractions from our research. I thank the office staff including Blazenka, Anna and Sylvie for always being impossibly helpful with any questions and humouring me when I was unable to fill out forms on my own. I would also like to thank Nagui and Scott for sharing their technical expertise as well as providing more powerful servers when my simulations became too large and onerous for a laptop.

Lastly, it is with great pleasure that I thank my family, friends and roommates for all of their support throughout this process. Thank you for your understanding when I was too busy, stressed or frustrated to go out or see you. Thank you for your encouragement and confidence when I didn't think this could be done. Thank you 
for listening and pretending to care when I was venting about computer problems, debugging errors or describing my current impasses.

Thank you. 


\section{Table of Contents}

$\begin{array}{ll}\text { Abstract } & \text { iii }\end{array}$

Acknowledgments $\quad$ iv

Table of Contents vi

List of Tables $\quad$ xi

List of Figures xiv

List of Acronyms xxiv

List of Symbols $\quad$ xxv

1 Introduction 1

1.1 Partial differential equations . . . . . . . . . . . . . . 1

1.2 Solving PDEs with a mesh . . . . . . . . . . . . . . 4

1.2.1 Finite difference method . . . . . . . . . . . . 4

1.2 .2 Finite element method . . . . . . . . . . . . . 6

1.2 .3 Boundary element method . . . . . . . . . 8

1.2.4 Problems with meshes . . . . . . . . . . . . . 9

1.3 A meshless alternative . . . . . . . . . . . . . . . 10

1.4 Objectives and contributions . . . . . . . . . . . . . 12 
2 Meshless methods $\quad 15$

2.1 Meshless advantages . . . . . . . . . . . . . . . 16

2.2 Solution formulations . . . . . . . . . . . . . . 17

2.3 Weak and strong formulation procedures . . . . . . . . . . 18

2.4 Shape function approximation schemes . . . . . . . . . . . . . 19

2.4.1 Smoothed particle hydrodynamics . . . . . . . . . . . 21

2.4.2 Moving least squares and kernel methods . . . . . . . . . . 24

3 Finite cloud method $\quad 26$

3.1 Formulation . . . . . . . . . . . . . . . . 26

3.1.1 Derivatives of the shape function . . . . . . . . 31

3.1.2 Kernel function \& kernel fixing . . . . . . . . . . . . . . 32

3.2 Previous FCM work . . . . . . . . . . . . . . . . 33

3.3 Diffusion example . . . . . . . . . . . . . . . . . . . . . 34

4 Improvements and initial tests $\quad 38$

4.1 Improvements in implementation . . . . . . . . . . . . . . 38

4.1 Modified FCM $\ldots \ldots \ldots . \ldots . \ldots 38$

4.1 .2 Node Scaling . . . . . . . . . . . . . . . . . . . . 44

4.1 .3 Node scaling . . . . . . . . . . . . . . . . . . . . . . . 44

4.1 .4 Cloud fixing . . . . . . . . . . . . . . . 46

4.1.5 Solution interpolation . . . . . . . . . . . . . . . 48

4.1 .6 Multi-threading ... . . . . . . . . . . . 49

4.2 Implementation . . . . . . . . . . . . . . . 50

4.2 .1 Basic geometry element creation . . . . . . . . . . 51

4.2 .2 Merging shapes . . . . . . . . . . . . . . . 54

4.2.3 Using Atar models for point generation . . . . . . . . . . . . . 57

4.2.4 Atar build of large optical model . . . . . . . . . . . 60 
4.3 Initial tests ......................... 64

4.3.1 1-D Poisson equation ................ 64

4.3 .2 2-D Poisson equation . . . . . . . . . . . 67

5 Thermal diffusion models $\quad \mathbf{7 2}$

5.1 Materially inhomogeneous FCM . . . . . . . . . . . 73

5.2 Heat diffusion equation . . . . . . . . . . . . . . . . . . 78

5.3 Transient solutions . . . . . . . . . . . . . . 80

5.4 Initial examples . . . . . . . . . . . . . . . 82

5.4.1 Pseudo-1D steady state . . . . . . . . . . . . . 82

$5.4 .23 \mathrm{D}$ steady state heat flow .............. 84

5.4 .3 Transient heat flow . . . . . . . . . . . . 85

5.4 .4 Gallium nitride power amplifier .......... 87

5.5 Mesa structures . . . . . . . . . . . . . . . . . . . . . 89

5.5 .1 Basic mesa analysis . . . . . . . . . . . . . 89

5.5 .2 FCM mesa correction . . . . . . . . . . . . . . 99 90

5.5 .3 Mesa transient analysis . . . . . . . . . . . 93

5.6 Using FCM thermal models for simulation and TCAD . . . . . . . . . 94

5.6.1 Silica based Mach-Zehnder model . . . . . . . . . . . . . 95

5.6 .2 SOI based optical devices $\ldots \ldots \ldots 96$

5.6 .3 Model reduction compact modelling . . . . . . . . . . . 100

5.6 .4 Multiple models and linking . . . . . . . . . . . . . 101

5.6.5 Individual model simulation and reduction . . . . . . . . 103

5.6 .6 Linked model simulation . . . . . . . . . . . . . 106

5.6 .7 Integrated simulations $\ldots \ldots \ldots 110$

5.7 Summary . . . . . . . . . . . . . . . . . . . . . 114 
6 Wave equations 116

6.1 Scalar wave equation $\ldots \ldots \ldots \ldots \ldots \ldots \ldots$

6.1.1 Time stepping in wave equations $\ldots \ldots \ldots \ldots$

6.2 Schrodinger's equation $\ldots \ldots \ldots \ldots \ldots \ldots$

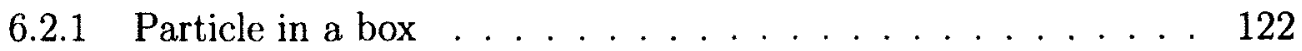

6.2 .2 Particle in a finite well . . . . . . . . . . . . . 124

6.2 .3 Particle in a parabolic well . . . . . . . . . . . 128

6.3 Maxwell's equations . . . . . . . . . . . . . . . 128

6.3 .1 Node placement . . . . . . . . . . . . . . . . . 131

6.3 .2 Boundary conditions . . . . . . . . . . . . . . 132

6.3 .3 Inhomogeneous solutions $\ldots \ldots \ldots \ldots$

6.3 .4 Basic eigenfrequencies . . . . . . . . . . . . . . 134

6.4 Summary . . . . . . . . . . . . . . . 136

$\begin{array}{lll}7 & \text { Mode solving } & 137\end{array}$

7.1 Mode solving . . . . . . . . . . . . . . . . . . 138

7.1 .1 Eigenmodes and eigenvalues . . . . . . . . . . . 139

7.1 .2 Step index method . . . . . . . . . . . . . . . . 141

7.1.3 Graded index method . . . . . . . . . . . . . . 143

7.1 .4 Remaining fields . . . . . . . . . . . . . . 145

7.1 .5 Symmetry . . . . . . . . . . . . . . . 146

7.2 Convergence and meshing $\ldots \ldots \ldots \ldots \ldots \ldots \ldots$

7.3 Guided mode tests . . . . . . . . . . . . . . . . 150

7.3 .1 Optical waveguides $\ldots \ldots \ldots \ldots \ldots \ldots \ldots$

7.3.2 Two microstructured waveguides $\ldots \ldots \ldots \ldots \ldots$

7.3.3 Metallic structures . . . . . . . . . . . . . . 159

7.4 Leaky boundary conditions . . . . . . . . . . . . . . 160 
7.4.1 Transparent boundary condition . . . . . . . . . . . 161

7.4 .2 Perfectly matched layer . . . . . . . . . . . . 164

7.5 Leaky mode results . . . . . . . . . . . . . . . . 167

7.5 .1 Air hole waveguide . . . . . . . . . . . . . 168

7.5.2 Annular air hole waveguide . . . . . . . . . . 171

7.6 Adaptive node refining . . . . . . . . . . . . . . . . . 175

7.6 .1 Examples and tests . . . . . . . . . . . 176

7.7 Summary . . . . . . . . . . . . . . . . . 182

8 Conclusion $\quad 184$

$\begin{array}{ll}\text { List of References } & 186\end{array}$ 


\section{List of Tables}

1 The error in the calculated first four eigenmodes for the simple eigenvalue test case. . . . . . . . . . . . . . . . . . 39

2 The error in the calculated first four eigenmodes for the simple eigenvalue test case. . . . . . . . . . . . . . . . . . . 43

3 Material thermal properties. . . . . . . . . . . . . . . 99

4 Model data: $S_{f}$ - full model size; $S_{r}$ - reduced model size; $T_{f}-\mathrm{cpu}$ time for full simulation; $T_{\text {red }}$ - cpu time to perform a model reduction; $T_{r s}-\mathrm{cpu}$ time to simulate a reduced model; $\mathrm{M}$ - Memory required for full simulation/model reduction; All times in seconds. Memory figures in gigabytes. Model size is number of unknowns. $\mathrm{A} \sim$ indicates the number was negligible. Solutions were obtained using MATLAB on a 64 bit Intel based PC with 12 cores running at $3.33 \mathrm{GHz}$ and 120 gigabytes of RAM. . . . . . . . . . . . . . . 106

5 Energies for the first four energy levels of the 1D particle in a box compared with known solutions and the corresponding percent error.

6 Energies for the first nine energy levels of the 2D particle in a box compared with known solutions and the corresponding percent error.

7 Energies for the first nine energy levels of the $3 \mathrm{D}$ particle in a box compared with known solutions and the corresponding percent error. 
8 Wave function and energy for the first 3 energy levels of the 1D particle in a finite, $3 \mathrm{eV}$, well with effective mass in the well $m_{W}=0.067$ and barrier effective masses of $m_{B}=0.067$ and $m_{B}=0.15$, compared with known solutions [1], showing the center 200 points of a total 450 points used in the simulation.

9 Comparison of effective index of refraction for the first six modes of the ridge waveguide. The s-FCM and g-FCM being the step-index FCM and the graded-index FCM, compared with results from Rsoft FemSIM

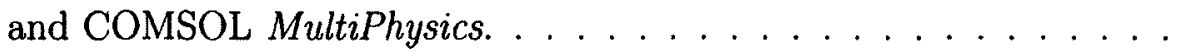

10 Comparison of effective index of refraction for the first six modes of the step index solid core waveguide. The s-FCM and g-FCM being the step-index FCM and the graded-index FCM, compared with results from Rsoft FemSIM and COMSOL MultiPhysics. . . . . . . . . . . 153

11 Comparison of effective index of refraction for the first six modes of the step fiber waveguide. The s-FCM and g-FCM being the step-index FCM and the graded-index FCM, compared with half and quarter symmetry using PEC and PMC boundaries. *Simulation time using a dual core iMac at $2.4 \mathrm{GHz} \ldots \ldots \ldots \ldots \ldots$

12 Comparison of effective index of refraction for the first six modes of the Bragg diffraction air core structure. The s-FCM and g-FCM being the step-index FCM and the graded-index FCM, compared with results from Rsoft FemSIM and COMSOL MultiPhysics. . . . . . . . . 156

13 Comparison of effective index of refraction for the first six modes of the air hole waveguide. The s-FCM and g-FCM being the step-index FCM and the graded-index FCM, compared with results from Rsoft FemSIM and COMSOL MultiPhysics. . . . . . . . . . . . 156 
14 Comparison of effective index of refraction for the first six modes of the air hole waveguide. The s-FCM and g-FCM being the air-hole FCM and the graded-index FCM, compared with half and quarter symmetry using PEC and PMC boundaries. . . . . . . . . . . . 158

15 Comparison of the real component of the effective index of refraction for the first two modes of the lossy ridge waveguide. The s-FCM and gFCM being the step-index FCM and the graded-index FCM, compared with results from Rsoft FemSIM and COMSOL MultiPhysics. Second, the loss component in $\mathrm{dB} / \mathrm{cm}$ of the waveguide compared with the same solvers. . . . . . . . . . . . . .

16 Comparison of effective index of refraction for the first six modes of the circular air holephotonic crystal fiber. The TBC and PML solutions are compared with results from a previously published FEM simulation.170

17 Comparison of effective index of refraction for the first six modes of the annular ring air hole fiber. The TBC and PML solutions compared with results from a FEM simulation. . . . . . . . . . . . . 175 


\section{List of Figures}

1 An example Finite Difference Mesh. . . . . . . . . . . . . 5

2 An example Finite Difference Mesh using slightly irregular grid meshing. Higher density points in the centre with a lower density near the

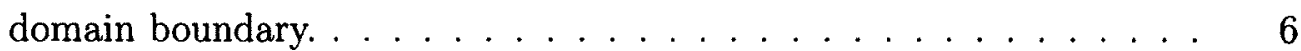

3 An example Finite Element Mesh, created using COMSOL Multiphysics [2]. . . . . . . . . . . . . . . . . . . 7

4 An example Boundary Element Mesh. . . . . . . . . . 8

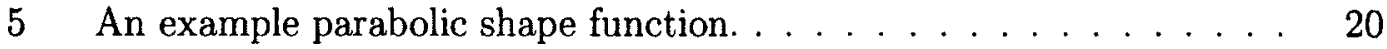

6 An example kernel function using an exponential weighting, both axes

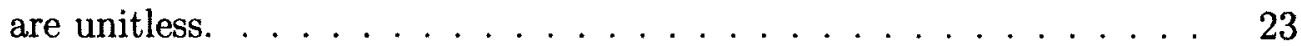

7 An example set of nodes in two dimensions and an irregular distribution. Also shown are the clouds for two nodes of interest. The clouds can be of different shapes and sizes depending on the local node dis-

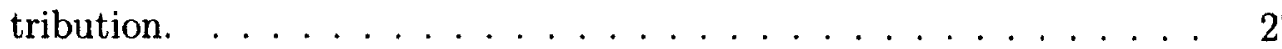

8 First four eigenmode solutions along with their mirrored counterparts for the Dirichlet eigenvalue test case. . . . . . . . . . . . . . . 40

9 Condition number for the moment matrix for all clouds in domain $0<x<1000 \ldots \ldots \ldots \ldots \ldots \ldots \ldots \ldots$ 
10 (a) Condition number for the moment matrix for all clouds in domain $0<x<1000$ with and without the translational fix. (b) First four eigenmode solutions along with their mirrored counterparts after the translational fix. . . . . . . . . . . . . . . . . . . . . .

11 Condition number for the moment matrix for clouds with a varying $d x$

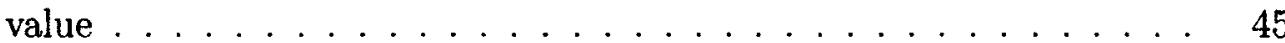

12 Point distribution for the cloud fixing example with an area of high point density directly touching an area of low density. Points are colour coded from blue to red for condition numbers 0 to 2.0 , points with a moment matrix condition number above 2.0 are shown in green. An initial unfixed cloud with a poor condition number is shown by the green box.

13 Example of the fundamental three dimensional shapes used in the FCM geometry creation engine. $\ldots \ldots \ldots \ldots \ldots \ldots$

14 Example of the fundamental two dimensional shapes used in the FCM geometry creation engine. . . . . . . . . . . . . 53

15 Example of an arc with different boundary node densities for the different sides, double row on the outer radius, single row on the inner radius and maximum phi angle and no extra points for the minimum phi angle boundary. . . . . . . . . . . . . . . .

16 Example of an arc with a linearly varying density, from high density at the inner radius to lower density at the outer radius. . . . . . . 54

17 Example of the alternate inner shape point distribution options for a disc. (a) cartesian (b) cartesian with random 'jiggle' added (c) purely random with a minimum allowed distance between points. . . . . . 54

18 Example of two inner shape point symmetry options for a disc, (a) fourfold symmetry (b) threefold symmetry. . . . . . . . . . . 55 
19 Example of (a) a polygon and (b) an arc with differing boundary conditions on each of its four edges. . . . . . . . . . . . .

20 The first shape in a geometry mapping file with the boundary normals shown. . . . . . . . . . . . . . . 56

21 The second step in mapping out nodes, removing any points within the area of the second shape listed. . . . . . . . . . . . . 5

22 Example of the final node mappings for a geometry file containing two discs, showing the points coloured according to their associated material and the boundary and interface points as well as their normals. 58

23 Example of an Atar model and quad-tree mesh. . . . . . . . . . . . . 59

24 Atar mesh transition for a homogeneous material. (a) Bad cloud due to double transition (asymmetrical point distribution). (b) Three typical clouds shown for good transition. Red/Blue/Green nodes and associated clouds. Transition nodes shown in black. . . . . . . . . . . . . 60

25 Atar mesh material transition. Red nodes are additional nodes added at the interface. The two clouds associated with one of the interface nodes are shown. .................. 6

26 FCM build of semi-circular waveguide with heater and thermal via. Atar build of substrate showing quad-tree mesh. (a) Top view. (b) Bottom view. (c) FCM arc geometry consisting of gold and silicon arcs. (d) FCM point distribution derived from Atar quad-tree mesh with nodes removed for addition of arc nodes. . . . . . . . . . 62

27 FCM point distribution for semi-circular waveguide. (a) All points. Surface and points. (b) Top view. (c) Bottom view. (d) Detail of point distribution for heater. . . . . . . . . . . . 63

28 Exact and computed solution for the one dimensional Poisson equation. 65 
29 Convergence of the FCM for the one dimensional Poisson equation, with $h$ the internode spacing. . . . . . . . . . . . . 66

30 Exact and computed solution for the one dimensional Poisson equation with a region of high solution gradient. . . . . . . . 67

31 Convergence of the FCM for the one dimensional Poisson equation with a region of high solution gradient, with $h$ the internode spacing. . . . 68

32 Exact, (a), and computed, (b), solution for the two dimensional Laplace equation. . . . . . . . . . . . . . . . . . . . . . 69

33 Convergence of the FCM for the two dimensional Laplace equation with $h$ the internode spacing. . . . . . . . . . . . 69

34 Exact, (a), and computed, (b), solution for the two dimensional Poisson equation with a region of high solution gradient. . . . . . . 71

35 Convergence of the FCM for the two dimensional Poisson equation with a region of high solution gradient with $h$ the internode spacing. .

36 Nodes separated into two materially different regions, having interface nodes and two clouds, each extending into only one region. . . . . . . 74

37 Diagram model for the simple homogeneous heat problem, with power added to darkened region in the middle. . . . . . . . . . . 83

38 Temperature profile along the $\mathrm{x}$-axis for a homogeneous single block of dimensions $(15 \mathrm{~m} \times 5 \mathrm{~m} \times 5 \mathrm{~m})$ with $25 \mathrm{~W}$ of heat added to the mid section and a conductivity of $\kappa=1 \mathrm{~W} / \mathrm{m}-\mathrm{K} \ldots \ldots$. . . . . . . 83

39 Diagram of the second heat problem for the inhomogeneous case of two materials. The two regions with different conductances are shown, with power added to the darkened region in the middle. . . . . . . . .

40 Temperature profile along the $\mathrm{x}$-axis for the inhomogeneous block of dimensions ( $21 \mathrm{~m} \times 5 \mathrm{~m} \times 5 \mathrm{~m}$ ) with two different material conductivities and a heat source of $25 \mathrm{~W}$ in the middle. . . . . . . . . . . . . 84 
41 3D heat flow in rectangular solid. (a)Atar model (Half view). Meshless model. (c)Atar heat contours (Half view). (d) Meshless heat contours. (e) Temperature through block in $\mathrm{z}$ direction. . . . . . . . .

42 (a) Node placement for the simple transient analysis with pink nodes the adiabatic boundary points, red being the Dirichlet nodes, grey the internal nodes, and yellow nodes are identified as the heat generating points. (b) Transient response for both the Atar solution and the FCM

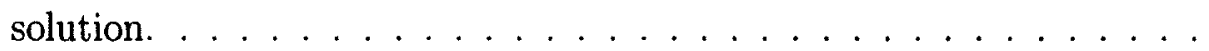

43 (a) Depiction of the 3D gallium nitride power amplifier used to compare the FCM results with Atar results. Red bars indicate locations of added power, with $2.4 \mathrm{~mW}$ added in each 'finger'. (b) Heat temperature plot showing FCM simulation results of amplifier. (c) Temperature profile comparison of the meshless and meshed methods for a $1 \mathrm{D}$ slice along the $\mathrm{x}$-axis through the centre of the amplifier. . . . . . . . 88

44 Mesa Structure. (a) Original course Atar mesh. (b) FCM point distribution. (c) Thermal distribution from FCM. . . . . . . . . . . . . 8

45 Mesa structure temperatures for both Atar and Atar-FCM. (a) Temperature along a vertical line through the centre of the mesa. (b) Temperature error, three different structures are plotted (see Fig. 46). 91

46 Atar mesh and Atar-FCM point distributions for a three sided mesa with three levels of refinement: (a) FCM-1. (b) FCM-2. (c) FCM-3. 91 
47 (a) Finite Cloud structure at Mesa Edge. The red node is the node of interest. Blue nodes are associated with the cloud for the mesa region. Green nodes are associated with the substrate cloud. Two nodes are shared at the interface. Yellow nodes are associated with neither cloud. (b) Temperature error versus mesa thermal resistance. The black symbols are the result of using an area weighting factor of 1.0 (the unadjusted case). The green symbols show the effect of using an area weighting factor of 0.85 giving an error below $0.6 \%$. . . . (a) Node placement for the simple transient mesa analysis with pink nodes the adiabatic boundary points, red being the Dirichlet nodes, grey the internal nodes, and yellow nodes are identified as the heat generating points. (b) Transient response for both the Atar solution and the FCM solution. . . . . . . . . . . . . . . .

49 Silica based Mach-Zehnder Device. (a) Cross-section of optical waveguide with heater element. (b) Layout of two semi-circular MZ with heaters placed on the outer arms. (c) Atar model of $1 / 2$ of a $\mathrm{MZ}$ element with additional thermal backside via $[3,4] \ldots \ldots \ldots$

50 Models built using FCM and Atar. (a) Optical ring based modulator with depletion modulation and thermal tuning. (b) Cross-section of ring based optical switch. (c) Cross-section of InP Disc laser. (d) Thermal contours for a standalone simulation of the disc laser model.

51 Thermal contours for circular waveguide. Active cooling of thermal via. (a) No power in heater. (b) Powered heater. (c) No cooling of thermal via but with power in the heater. (d) Thermal transient for heater power transitioning from off to on (11 points distributed along arc) . . . . . . . . . . . . . . . . . . . . 104 
52 Linked model simulation. (a) Complete linked model with all three devices. (b) Temperature profile for linked model. . . . . . . . . . . . 107

53 Temperatures in models. (a) Temperature rise around the circular waveguide in the Ring Switch and the Modulator $(R=0$ is on the left hand side of the two structures and increases counter-clockwise). (b) Temperature rise along the straight waveguide section. Full simulation results are shown as solid lines; reduced simulation results as symbols. Both models with and without metalization are shown. . . . . . 108

54 Linked model simulation with a more complete metalization. (a) Complete linked model with all three devices. (b) Temperature profile for linked model. . . . . . . . . . . . . . . . . . . . . . 109

55 Schematic of simulated system comprised of a laser, modulator, switch and two detectors and a distributed thermal model. . . . . . . . . . 112

56 System response. System is tuned to pass the data to the drop port. The drop port response is shown in black and the through port response in red. Top plot shows simulation using temperature from the nominal model. Bottom plot presents a simulation for temperatures from the model with metalization present. . . . . . . . . . . 113

57 First four eigenvectors from the 1D particle in an infinite box of $0.5 \mathrm{~nm}$ width, 100 points, node values shown as the points and theoretical known solution as the solid line. . . . . . . . . . . . . . . . 123

58 Solution to the 2D particle in a box simulation showing the eigenvector $\left.\psi_{(} 4,2\right)$ for an infinite box of dimensions $1 \mathrm{~nm} \times 0.5 \mathrm{~nm}$. . . . 125

59 Energies for the first 3 energy levels of the $1 \mathrm{D}$ particle in a finite $3 \mathrm{eV}$ well with effective mass in the well $m_{W}=0.067$ and barrier effective

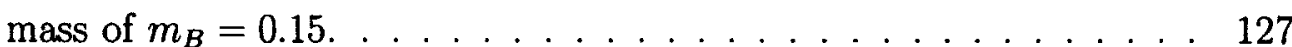


60 Wave function shown at 44 discrete time steps through both extremes of a one dimensional parabolic well, also shown. . . . . . . . . . . 129

61 A Yee cell showing the locations of the field vectors being represented in a staggered grid. . . . . . . . . . . . . . . . . 132

62 An illustration of a field vector from a node, and the nine points surrounding the plane normal to the vector which are used to calculate the permittivity node and direction. . . . . . . . . . . . . 133

63 Single sided amplitude spectrum for the one dimensional EM random excitation of a $10 \mathrm{~m}$ line in free space with Dirichlet boundary conditions and the expected oscillation frequencies in black for reference.

64 Single sided amplitude spectrum for the two dimensional EM random excitation of a $0.1 \mathrm{~m} \times 0.1 \mathrm{~m}$ square in free space with reflective boundary conditions and the expected oscillation frequencies in black for

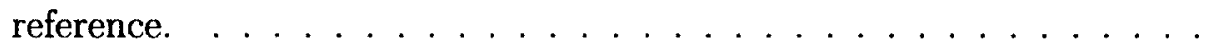

65 (a) An example cloud from the stitched method with nodes separated into two regions, showing interface nodes and two 'half' clouds, each extending into only one region. (b) An example cloud using the graded method with all nodes in one continuous region and a single cloud over a varying material property. . . . . . . . . . . . . . . . . . . . 144

66 Parameters and dimensions for a solid core step-index fiber used for convergence studies, showing the entire solution domain with an example point layout. . . . . . . . . . . . . . . . 147

67 First six modes of the step index fiber. . . . . . . . . . . . . . . 148

68 Convergence of the first mode of the step index fibre for both the gFCM and s-FCM methods plotted against total number of points using a constant density radial distribution. . . . . . . . . . . . . 148 
69 Alternate point distribution for the step index fiber with a point density ratio of 1:4 for the core center to interface (yellow points), and a ratio of $16: 1$ for the interface to outer cladding (blue points). . . . . . .

70 Convergence of the first mode of the step index fibre for both the gFCM and s-FCM methods plotted against total number of points using a point density ratio of $1: 4$ for the core center to interface and a ratio of $16: 1$ for the interface to outer cladding. . . . . . . . . . . 150

71 Parameters and dimensions for the ridge waveguide. . . . . . . . . 151

72 The first six modes of the ridge waveguide . . . . . . . . . . . . 152

73 Parameters and dimensions for (a) a Bragg diffraction air core fiber and (b) a photonic crystal fiber with six circular air holes. . . . . . 155

74 The first six modes of the Bragg diffraction air core structure. . . . . 157

75 The first six modes of the circular air hole photonic crystal fiber. . . . 157

76 Dispersion parameter comparison between COMSOL MultiPhysics and the FCM for modes 1 and 3 of the photonic crystal fiber. . . . . . . 159

77 Parameters and dimensions for the lossy ridge waveguide with gold on either side of the ridge. . . . . . . . . . . . . 160

78 Three different regions of the PML . . . . . . . . . . . . . 165

79 Imaginary component of the index of refraction for the fundamental mode of the air hole fiber with a TBC at a varying diameter. . . . . . 169

80 Imaginary component of the index of refraction for the fundamental mode of the air hole fiber with a PML at (a) varying depth, and (b) varying width. . . . . . . . . . . . . . . 169

81 The first six modes of the circular air holephotonic crystal fiber using a square PML. . . . . . . . . . . . . . . . 171

82 Parameters and dimensions for the annular ring air hole fiber. . . . . 172 
83 Imaginary component of the index of refraction for the fundamental mode of the annular air hole fiber with a TBC at a varying diameter. 173

84 Imaginary component of the index of refraction for the fundamental mode of the annular air hole fiber with a PML at (a) varying depth, and (b) varying width. . . . . . . . . . . . . . 174

85 The first six modes of the annular ring air hole fiber using a square PML.174

86 (a) A coarse node mapping of the step index fiber, with core nodes (yellow) and cladding nodes (blue). (b) field magnitude for the fundamental mode . . . . . . . . . . . . . . . . . 177

87 Histogram of the magnitude of the rate of change of the $\mathrm{H}$ field for the points in the coarse step index fiber fundamental mode solution, values normalized to the maximum gradient. . . . . . . . . . . 178

88 New node placement after one iteration of the adaptive refinement for the step-index fiber. New and cladding nodes (blue) original core nodes (yellow). . . . . . . . . . . . . . . . .

89 (a) A coarse node mapping of the air hole photonic crystal fiber, with photonic crystal nodes (red) and air holes nodes (blue). (b) field magnitude for the fundamental mode . . . . . . . . . . . 180

90 New node placement after one iteration of the adaptive refinement for the air hole photonic crystal fiber. New and photonic crystal nodes (red) air hole nodes (blue). 


\section{List of Acronyms}

\begin{tabular}{ll}
\hline Acronym & Definition \\
\hline \hline ABC & Absorbing Boundary Condition \\
BC & Boundary Condition \\
BCM & Boundary Cloud Method \\
BEM & Boundary Element Method \\
DE & Differential Equation \\
FCM & Finite Cloud Method \\
FDM & Finite Difference Method \\
FDTD & Finite Difference Time Domain \\
FEM & Finite Element Method \\
MEMS & Microelectromechanical Systems \\
MLS & Moving Least Squares \\
MR & Model Order Reduction \\
MZ & Mach-Zehnder \\
ODE & Ordinary Differential Equation \\
PDE & Partial Differential Equation \\
PEC & Perfect Electric Conductor \\
PMC & Perfect Magnetic Conductor \\
PML & Perfectly Matched Layer \\
SPH & Smoothed Particle Hydrodynamics \\
TBC & Transparent Boundary Condition \\
\hline &
\end{tabular}




\section{List of Symbols}

\begin{tabular}{ll}
\hline Symbols & Definition \\
\hline \hline$C$ & thermal capacitance, speed of wave in scalar wave equation \\
$\varepsilon_{r}$ & relative permittivity \\
$\varepsilon_{0}$ & vacuum permittivity \\
$\hbar$ & electric field \\
$H$ & Planck's reduced constant \\
$\kappa$ & magnetic field \\
$m_{B}$ & thermal conductivity \\
$m_{e}$ & effective mass of an electron in a barrier \\
$m_{W}$ & mass of an electron \\
$m_{e f f}$ & effective mass of an electron \\
$\Psi$ & wave function \\
$R$ & reflectance parameter for a PML \\
$R_{t h}$ & thermal resistance \\
$\rho$ & heat source \\
$\sigma$ & thermal capacitance, electrical conductance \\
$T_{r i s e}$ & rise in temperature \\
\hline
\end{tabular}




\section{Chapter 1}

\section{Introduction}

The field of Engineering has a need to accurately model physical phenomena and to understand how devices will operate and behave. As these devices become more complex and intricate, the models and modelling tools must also increase in accuracy, functionality and ease of use. Increasing speeds and processing power of computers to perform these simulations has enabled more intricate designs while reducing the computational solving time. The onus is thus increasingly being put on the creation of these tools, and the development of the models.

\subsection{Partial differential equations}

Many of the problems faced for such tools are partial differential equations (PDEs), which are found to accurately model physical phenomena such as heat flow, wave propagation, and many others. Differential equations (DEs), unlike straightforward algebraic equations, relate a function to its derivative, as an example, $u^{\prime}(x)=u(x)$. Two groups of differential equations are ordinary differential equations (ODEs) and partial differential equations (PDEs). An ODE involves the derivative of a single variable, $x$ in the previous example. A partial differential equation will include partial 
derivatives and several variables such as $u_{t}(x, t)=u_{x x}(x, t)$. The notation $u_{t}$ or $u_{x x}$ is used as shorthand for $\frac{\partial u}{\partial t}$ and $\frac{\partial^{2} u}{\partial x^{2}}$, respectively. The differential equation is also classified as being of a certain order, given from the highest derivative contained within. The order of the previous two examples are first and second, respectively [5].

A homogeneous differential equation includes only terms which depend on the unknown function, such as $u$ in the previous examples. An equation including terms which do not depend on the unknown function is termed non-homogeneous. An example of a non-homogeneous equation is $u_{t}(x, t)-u_{x x}(x, t)=f(x, y)$, with $f(x, y)$ being the added term sometimes called the forcing term.

A second source of complexity can be brought about by an inhomogeneous material domain or a spatially varying material parameter. The $1 \mathrm{D}$ heat flow equation,

$$
\frac{d}{d x}\left(\kappa(x) \frac{d T}{d x}\right)=f(x)
$$

where $\kappa(x)$ is the spatially varying thermal conductivity, is an example. This inhomogeneity can either be due to: 1) distinct regions of different materials and therefore constant but differing thermal conductivities, or 2) a smooth continuously varying thermal conductivity. In this thesis I will use the term inhomogeneous to refer to material parameter variation and non-homogeneous to refer to a differential equation which has an independent forcing or source term.

Boundary conditions are also an important aspect of DEs, and act to further constrain a solution. For example the solution of $u^{\prime}(x)=u(x)$ could be $u(x)=e^{x}$ or $u(x)=5 e^{x}$, whereas a boundary condition such as $u(0)=1$ would restrict the potential solutions. For the majority of solutions in this thesis two types of boundary conditions will be used, the Dirichlet and Neumann boundary conditions. Dirichlet boundary conditions, or first-type BCs, restrict the value of the function at certain 
points. The previous example, with $u(0)=1$, is a Dirichlet $\mathrm{BC}$. The other main $\mathrm{BC}$ used is the Neumann $\mathrm{BC}$, or second-type, which restricts the derivative of the function. An example of a Neumann $\mathrm{BC}$ could be $u_{x}(0)=0$. This particular condition is often implemented as it can be used to restrict the flow, or rate of change, at a boundary. As we shall see, more complex boundary conditions are sometimes needed, particularly when an infinite domain needs to be approximated for a wave-like equation and an absorptive boundary condition is needed.

Large physical models which can be characterized by PDEs quickly become very challenging to solve and require a sophisticated method and powerful computers to do so. To solve these models the solution domain is partitioned into smaller pieces, or elements, with the entirety of the elements referred to as a mesh. The mesh is designed in such a way as to numerically approximate the appropriate derivatives for the model using a "shape" function which describes the variation of a variable over an element of the mesh. This leads to the creation of a shape (or operator) matrix, $\mathbf{N}$ and a vector containing the system variables which are to be solved, $\mathbf{U}$.

In a discretized system the shape matrix, when multiplied by the unknown function, acts as an operator. A self replicating shape function would be an identity matrix which when multiplied by any function results in that same matrix, such as $\mathbf{N U}=\mathbf{I U}=\mathbf{U}$. With spatial knowledge of the discretized unknown function one can also create derivative operators for the shape function, eg. $\mathbf{N}_{\mathbf{x}} \mathbf{U} \Rightarrow \frac{d}{d x} \mathbf{U}$.

For a time independent solution the typical approach would involve the decomposition or inversion of the shape matrix, and appropriate matrix multiplications in order to determine the field variables. For example, given the equation $\frac{d^{2}}{d x^{2}} \mathbf{U}=\mathbf{B} \Rightarrow \mathbf{N}_{\mathbf{x} \mathbf{x}} \mathbf{U}=\mathbf{B}$, the solution would be $\mathbf{U}=\mathbf{N}_{\mathbf{x}} \mathbf{x}^{-1} \mathbf{B}$. With a time dependent solution one must repeatedly solve the system equation using a fixed or variable time interval using a time stepping method. Time stepping methods typically fall 
into two categories, that of implicit which requires a matrix inversion, and explicit which does not require inversion and uses simple matrix multiplications [6].

Both categories of time stepping methods have advantages and disadvantages, and several will be used in this thesis with full explanations and justifications for their use. Advantages for the different methods involve speed, accuracy, stability of solution, and memory required for the solution. As an example, a full vector Maxwell equation solution requires a matrix with dimensions six times larger than that of a scalar equation due to the electric and magnetic field vectors. Thus, matrix inversion can become quite costly in a vectorial three dimensional domain. For such large problems an explicit time stepping method is more practical by removing the need for a matrix inversion or decomposition.

\subsection{Solving PDEs with a mesh}

The three main methods described below all require a mesh created using strict geometric rules and a priori shape function knowledge, and are thus dubbed 'meshed methods'. Each meshed technique also has its own strengths and weaknesses. These methods are the Finite Difference Method (FDM), the Finite Element Method (FEM), and the Boundary Element Method (BEM). Each method attempts to solve for the unknown field values in a domain of interest. The domain is the entirety of the modelling environment which is to be discretized and solved.

\subsubsection{Finite difference method}

The Finite Difference Method (FDM) is considered the most straightforward method that will be described and can be implemented rather easily. This method discretizes the given system into a regular grid and uses these grid differences to obtain a solution 
directly. The entirety of the grid is the mesh and the mesh spans the entire computational domain. The difference between the field at two neighbouring nodes can be divided by their distance to give an approximate slope calculation. This manipulation can be placed into the shape matrix as a derivative operator. An example of such a difference scheme for first and second order derivatives, using central-difference approximation is [7]

$$
\begin{aligned}
u^{\prime}\left(x_{0}\right) & =\left.\left(\frac{d u}{d x}\right)\right|_{x_{0}} \approx \frac{1}{2 h}\left(u\left(x_{0}+h\right)-u\left(x_{0}-h\right)\right) \\
u^{\prime \prime}\left(x_{0}\right) & =\left.\left(\frac{d^{2} u}{d x^{2}}\right)\right|_{x_{0}} \approx \frac{1}{h^{2}}\left(u\left(x_{0}+h\right)-2 u\left(x_{0}\right)+u\left(x_{0}-h\right)\right)
\end{aligned}
$$

with $h$ being the grid spacing and the derivative taken at location $x_{0}$.

An example of an FD mesh is shown below in Fig. 1. This mesh is regular using constant grid spacing, however it is possible to manipulate the mesh to give regions of higher and lower density. Typically this irregular meshing will create more nodes than necessary along the coordinate axes, as seen in Figure 2. The standard grid is also restricted to a rectilinear layout, with approximation schemes and very high density required to mimic curved contours. These can add significantly to the number of nodes and computational effort needed to solve a problem.

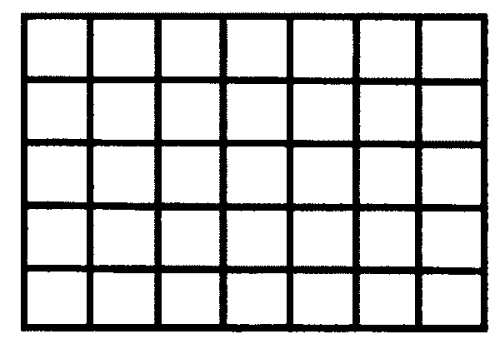

Figure 1: An example Finite Difference Mesh.

The use of irregular grids is possible, however the model does not lend itself well to such scenarios, and can quickly become arduous for the mesh creation. Research 


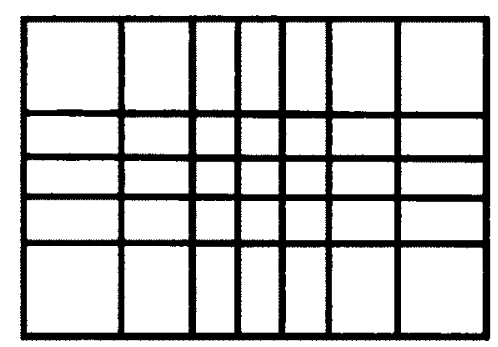

Figure 2: An example Finite Difference Mesh using slightly irregular grid meshing. Higher density points in the centre with a lower density near the domain boundary.

in the area of irregular FD grids is ongoing [8].

\subsubsection{Finite element method}

The finite element method began appearing in the 1940's to aid in the modelling of torsion problems for structural mechanics, with the term finite element originating in 1960 [9]. The domain to be solved by the FEM is divided into a highly irregular set of subregions termed finite elements, with the entire domain of finite elements being the mesh.

An example mesh is shown below, Fig. 3, demonstrating the typical usage of triangular shapes in two dimensions. For three dimensions tetrahedrons or hexahedrons are common. As the shapes of the elements are known and their geometric relations to neighbouring elements follow strict rules, it is possible to create equations approximating their areas and their relations to neighbouring shapes. These rules and shape approximations are used to approximate path integrals and differentials throughout the computational domain. A shape function or matrix can be constructed, and the governing equation adapted to use this numerical approximation to solve the problem [10].

Since FEM can use a highly irregular mesh it has many advantages over the FDM, including the ability to create areas of high and low density tailored to the needs of the 


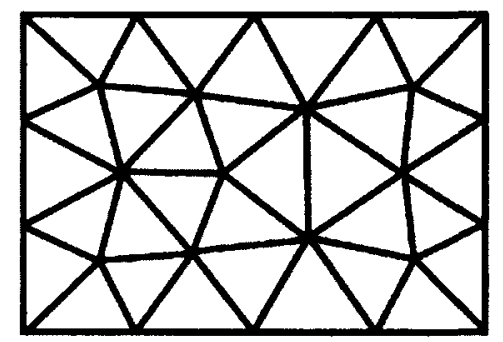

Figure 3: An example Finite Element Mesh, created using COMSOL Multiphysics [2].

model. Its limitations become more apparent with the creation of high quality meshes in intricate shapes and domains. Automatic mesh generation can be quite successful, however automated high quality meshes are still quite difficult to obtain in higher dimensions, and adaptive meshing of hexahedrals in three dimensions is not reliable. The creation of automatic mesh generation tools can also be quite challenging and requires considerable overhead and effort for even basic meshes with easily generated meshes being of naturally poor quality [11].

Further problems can result from the use of finite elements. In crack growth type problems the cracks are restricted to the edges of the elements, thus the results are inherently based upon the initially created mesh. Strain type problems are also affected as with large amounts of strain the elements can become deformed. The shape functions are based on specific relations and geometries of the elements and can become invalid with too large a deformation. These issues can be aided by remeshing after subsequent iterations or through the use of adaptive meshing.

Adaptive meshing, however, requires the mapping of field variables repeatedly during the solution process which can add considerable computational cost and lower accuracy. As well, for a large three dimensional problem this remeshing at each step can become very onerous and demanding on CPU time. 


\subsubsection{Boundary element method}

The boundary element method is the third method to be discussed and is also the most recently developed of the three main methods for solving PDEs. Practical tools using the method arose in the 1970's and are used to solve boundary integral equations as well as ODEs changed into equivalent ordinary integral equations [12].

In its simplest form the boundary of a domain is discretized into elements with the unknown function presumed constant on each element. The boundary integral equation can then be readily adapted to include piecewise constant elements and a shape matrix can be formed, from which the usual decomposition and solving can take place. More complicated meshes using elements with linearly or otherwise nonconstant unknown values have been created and are commonly used. An example BEM mesh is seen below in Fig. 4.

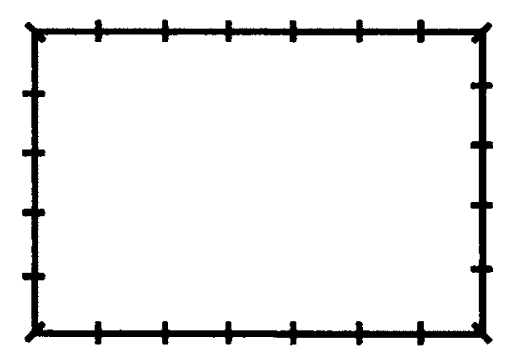

Figure 4: An example Boundary Element Mesh.

BEM has as a benefit over FEM that it allows for a reduction in the dimensionality of the solution domain, such as from three dimensions to two dimensions. The solutions found using BEM for many cases tend to zero as the domain grows to infinity, removing the need of a very large domain truncated with an absorbing boundary condition $(\mathrm{ABC})$. The mesh used in BEM does not require the continuity boundary conditions which restrict FEM meshes, making a remesh or adaptive mesh easier to implement by simply replacing a portion of the boundary mesh with a finer discretization [13]. One issue that arises with BEM is that the method produces large 
dense operator matrices, unlike FDM and FEM which produce larger but sparse matrices. Computational operations needed to solve dense matrices scale significantly faster than with sparse matrices [6].

Another disadvantage of the BEM is the difficulty in solving problems with intricate volumetric definitions in the interior. For example, a heat flow problem with a complex heat generation region would need internal meshing, removing any benefits of a simple outer boundary.

\subsubsection{Problems with meshes}

All three of these methods have been highly researched and used in both academic and industrial environments. A significant drawback to each of these methods is the effort needed to produce the mesh required to form a solution.

FDM has a highly regular grid, typically with the same grid density over the entire domain. This creates a very large number of points for even simple geometries and is undesirable. Densities can be changed by the merging of grids however creating these algorithms and working on varying models can make the process quite difficult. As well the geometries are restricted to rectilinear layouts requiring approximations and many additional points for curvilinear models.

FEM can handle large changes in element densities and is better able to suit curvilinear geometries than FDM. Extensive geometric knowledge of the domain must be known and algorithms for meshing the domains must be quite advanced. The meshing process of creating and fitting the predetermined shape elements can be quite difficult to code and automated meshing for complex geometries is generally not of the highest quality. PDEs solving for stress and strain break down as the elements become distorted and no longer accurately approximate their own shapes. Fracture and point of failure tests are restricted to the interfaces in the predetermined elements. 
Remeshing the domains in an iterative manner is possible however the solution quality degrades with the interpolations. The remeshing is also a computationally intensive procedure as one cannot simply add more triangles on top as the geometric relations and elements must be recalculated. Adaptive meshing is quite difficult with FEM and meshed based solutions, particularly in three dimensions.

BEM solutions typically form dense operator matrices, unlike the sparse matrices formed by FDM or FEM. Such matrices are computationally much more difficult to decompose than their sparse counterparts.

\subsection{A meshless alternative}

A more recent group of methods used to solve PDEs, which aim to address the meshing issues, is dubbed meshless or meshfree methods. These methods use a series of nodes and node generation instead of a mesh and require little geometry or a priori knowledge of the domain for their creation.

Nodes are simply zero dimensional points which are distributed throughout the computational domain. Each node represents the field to be solved or an approximation of the field, such as an integral of the field in the local area. These points replace the square elements of the FDM and the triangles or tetrahedrons in the FEM. Each point also has a domain of influence, or a finite cloud, surrounding it which encompasses neighbouring points.

Original meshless methods were published in the 1970's based on the method of smoothed particle hydrodynamics with applications primarily to astrophysics and then later adapted to materials and solid mechanics. Another type of meshless method being developed at the time was the finite difference method using arbitrary irregular grids and a generalized approach. These methods were initially successful although 
suffered from problems with stability and accuracy.

Meshless or point methods can have a variety of advantages over meshed methods. Initially, the creation of a computational domain can be as easy as distributing a number of points or nodes over an area. There is no need to fit triangles or other shapes together or to follow any geometric pattern or relations. This is attractive as less time needs to be spent in the mesh generation code.

Iterative or adaptive node placement techniques are better suited to meshless techniques as they allow for the easy placement of new nodes in any desired region without a need to remesh the entire domain. There is no restriction that a new node fit into the larger elements or shapes, and no need to calculate the geometric relations and requirements for the new node. A simple shape recalculation for the areas, or clouds, surrounding the newly added nodes is required.

Large deformations in stress or strain problems are not an issue as the shape functions are created at runtime and can be recalculated for specific regions with little extra effort and no remapping.

Due to the nature of the method one can obtain information on the accuracy of the node placement and shape functions and make improvements prior to obtaining a solution. As well, the interpolation functions for the behaviour of a solution between points are calculated during the solution process. These functions can then be used to both interpolate the solution as well as trivially find solution differentials throughout the computational domain.

A detailed description of meshless or meshfree methods is provided in Chapter 2, including their benefits, different formulations, and problems previously solved using these methods. This thesis proposes the use of a particular meshless method, the Finite Cloud Method (FCM) and involves advanced research into the method and its application to a variety of engineering problems. The FCM, fully explained in 
Chapter 3, uses a domain of nodes and a cloud of neighbouring nodes to create shape functions from which the solution to the problem can be obtained. The lack of mesh or intricate geometry allows for easy and highly irregular node placement, adaptability and low overhead. The implementation of the FCM along with improvements to the method are detailed.

The remaining thesis explores the research and problems that have been solved using the FCM, which to my knowledge has not previously been done. Chapter 5 will explore the use of the FCM in solving materially inhomogoneous problems beginning with the heat diffusion equation. Solutions for both time dependent and time independent examples are provided along with comparisons to theoretical and commercial solvers. As well, a method of model reduction and model linking is presented for solving complex opto-electronic circuit simulations. Several wave equations are briefly explored for use with the FCM in Chapter 6, including Schrodinger's and Maxwell's equations.

An in depth analysis of fully vectorial coupled field equations is explored for the solution of eigenvector and eigenvalue modes of optical waveguides in Chapter 7. Waveguide mode solutions are provided for both guided and lossy modes using a variety of boundary conditions. An adaptive meshing scheme is also presented showing the ease of use and potential with the FCM.

\subsection{Objectives and contributions}

The objective of this thesis project is to investigate an alternative method of solving partial differential equations, with applications in electrical engineering, which does not use a mesh in its solution. By applying the method to a variety of equations, the method can be fully explored to discover improvements in implementation, and its 
benefits and disadvantages with respect to other solution methods.

To obtain this objective a ' $C$ ' program has been created which implements the finite cloud method and is used to solve PDEs. Initially the method was used to solve materially homogeneous problems and to replicate past FCM solutions seen in $[14,15]$. Having not seen an application of the FCM in solving materially inhomogeneous problems, I implemented a heat diffusion solver using the finite cloud method and devised a method for solving such inhomogeneous problems which was published in $[16]$.

The heat diffusion solving tool also proved useful for studying methods of combining rectilinear models with curvilinear models. An advanced node mapping engine was created to replace the mesh created in Atar with points as to be used with the FCM. The conversion has allowed verifying the accuracy of the FCM with respect to Atar and allowed for the creation of very large and complex finite difference models with additional curvilinear shapes. This led to an advanced application of the FCM by implementing model order reduction in the solution of optoelectronic circuit simulations, published in the IEEE transactions Journal of Technology Computer Aided Design [17]. It is shown in this paper that the FCM can be used in conjunction with model order reduction, which can significantly reduce the size of the governing matrices. The process is also used to join several reduced large models which allowed for the numerical solution of an otherwise intractable problem.

During this process several implementation improvements to the method were introduced, these are covered in Chapter 4.1. As these had not yet been seen by the author, transient and steady state solutions to wave equations were next attempted, including materially inhomogeneous quantum problems. The FCM was shown to be fully capable of solving these hyperbolic equations, and an initial investigation into Maxwell's equations shows some success. 
As the optics industry is continuously looking for better and easier tools to solve for optical modes, I next adapted the FCM to create a mode solving engine. This solution engine and a set of complex examples was presented at Photonics West 2012 [18]. A thorough look at solving fully guided modes in optical microstructured waveguides using the FCM was published in Optics Express [19]. I have also performed a detailed analysis of the FCM using absorbing boundary conditions for the solution of leaky modes, presented at Photonics North 2012 and an expanded version has been published in the IEEE/OSA Journal of Lightwave Technology [20].

A final contribution to the FCM is the use of adaptive node mapping in the solution process. Adaptive node placement is a highly sought after trait for PDE solvers and Chapter 7.6 details initial attempts and success in adding adaptive mapping to the mode solving engine.

The novel work is found in Chapters $4,5,6,7$ and the background material in Chapters 2 and 3. 


\section{Chapter 2}

\section{Meshless methods}

The descriptions in this chapter are an overview of meshless methods and follow the references $[11,21]$. Meshless methods are a relatively recent development in the search for a more accurate and efficient solution method to PDEs. The originating method was smoothed particle hydrodynamics (SPH), used for modelling astrophysical phenomena in the 1970's. This method was developed as an alternative to a finite difference solution which for the models required too large a number of points [22]. This method enabled the distribution of a very large number of points in an adaptive manner and allowed a highly variable point distribution. Such techniques are required for the vast scales used in astrophysics and solutions would not be possible with FD and a regular grid. For the following two decades minimal research was done on this method, apart from estimates on the kernel accuracy [23].

During this time research using finite difference method and arbitrary irregular grids was also ongoing, in particular with material engineering and solid mechanics. These meshed solutions for mechanical PDEs involving crack propagation are particularly difficult to solve as the cracks are forced to propagate along the shape elements. This problem can be helped by remeshing in subsequent steps, requiring projecting 
solutions between elements which degrades the accuracy, as well as adding significant computation time. Similarly, stress and strain problems with large deformations break down as the elements in a meshed solution become overly distorted.

In the mid 1990's research into meshless methods has grown significantly to help with these problems, and the problems of automatic or adaptive complex meshes mentioned in the previous chapter.

\subsection{Meshless advantages}

Meshless PDE solvers have several potential advantages over their meshed counterparts. Unlike with FDM solvers which are restricted to rectilinear layouts, the node placement has no rigid structure allowing the nodes to follow the contours of any objects, giving curvilinear structures.

The node distributions do not need to have any predefined shape either, unlike with FEM which must use a set of predefined shapes and intricate shape meshing to cover the entire domain. This process makes adding new geometries difficult as meshing algorithms must be created for the shape and its interconnections at junctions. Any change in the domain geometry requires a remeshing of the entire domain unlike with meshless methods which can simply remap the local area affected.

As well, meshless methods allow for the easy connection of differing models, potentially created from entirely different programs. Nodes from disparate models are simply placed together with any connecting nodes enforcing heterojunction boundary conditions. There is no need to be concerned about consistency conditions, geometry rules, or how to mesh the two structures together.

The method also lends itself quite well to adaptive node placement, whereupon solutions are determined using a limited number of points and a coarse distribution. 
Next, the initial solution is used as a mapping for further node additions, with more nodes being added in areas of high solution gradient and fewer or no nodes being placed in areas of low solution gradient. New nodes require a remapping of their domain of influence but must follow no geometrical rules unlike the remeshing required by the FEM.

Meshless methods present potentially straighforward point placement techniques and implementations for PDE solving. Further advances with intelligent mapping and adaptive mapping could result in a highly effective and accurate PDE solving tool. This ability to readily join disparate models allows for integration with other tools including both mapping and equation solving engines. These potential advantages provide the reasoning and impetus for further research into meshless methods.

\subsection{Solution formulations}

Meshless methods have two main formulation approaches for solving PDEs, a strong approach and a weak approach. It is also possible to combine these approaches into a weak-strong method which is considered out of the scope for this background chapter.

These methods can also be categorized by the method of creation of their shape functions which is a central issue to any meshless solver. The shape function is an interpolation matrix used in a discrete numerical solution to approximate the desired function, and thus a 'good' shape function is of utmost importance. Historically, the shape function methodologies have been divided into several main variations however these categories are interrelated and can be said to reduce to each other under certain circumstances or are built upon one another. It is possible to further subdivide the methods or to include several other special cases. However, due to their similarity these distinctions will be kept to a minimum and the overarching techniques 
themselves will be discussed.

\subsection{Weak and strong formulation procedures}

The solution of PDEs can come in three principle forms, a strong form, a weak form or a combination weak-strong form.

Weak form solutions provide a weak consistency condition for the numerical approximation and can thus be computationally easy to solve. The solution points are distributed over the entire domain, which is also covered by a background grid of cells or shapes. The weak method works by introducing an integral of a shape function over the small regions or background cells to capture the mathematical or physical properties of the equation. This set of background cells means that the weak-form solutions are not truly meshless. As the points represent an integral of the fields and not the fields themselves the system of equations to solve must be modified to form new governing equations. These can be used on complex systems to provide accurate and very stable results, with the process being similar to the shape elements and formulation methods of the FEM [21].

The strong form solution discretizes the PDE for both the domain and boundary into the field nodes, and the unknown values or variables are solved directly. Such a method has a similar representation as the meshed FDM which discretizes the governing equation into a grid and can then be solved at each node. The strong methods are considered truly meshless as there is no underlying grid or cell mapping, and are described as 'collocation techniques'. Such methods are typically easier to implement, understand, and can be computationally efficient. Historically, however, this type of solution can have problems with accuracy, stability, robustness, and derivative boundary conditions [21]. 


\subsection{Shape function approximation schemes}

Meshless methods can also be classified by their shape function approximation scheme, which for discrete numerical systems creates a shape matrix. The shape function is an interpolation or approximation function which acts as an operator and its proper construction method is critical for accurate solutions.

The generic shape function, $N$, can be used to interpolate a function $u(x)$ between the known data points. As an example, one can obtain the value of $u\left(x_{0}\right)$ by a summation over all $N P$ data points as

$$
u^{a}\left(x_{0}\right)=\sum_{I}^{N P} N\left(x_{I}-x_{0}\right) u\left(x_{I}\right)
$$

with $u^{a}$ an approximation of the function $u$.

An example of a simple shape function for a node at $x_{0}$ with inter node spacing $h$ would be a parabola with value $N(0)=1$ at the node of interest, and $N(x)=0$ for $|x| \geq h$, Fig. 5. This function is able to exactly represent the values of nodal positions, as well as approximately interpolate for any position between known nodes. The shape function can then be made into an operator function as $\frac{d N}{d x}$ which when

multiplied by the unknown function and summed as above results in $\frac{d u}{d x}$. This can be shown as

$$
\frac{d u^{a}\left(x_{0}\right)}{d x}=\sum_{I}^{N P} \frac{d N\left(x_{I}-x_{0}\right)}{d x} u\left(x_{I}\right)
$$

with the derivative of the function $u$ at point $x_{0}$ being approximated. A PDE can 
then be expressed using the above in the following manner,

$$
\begin{aligned}
u^{a}\left(x_{0}\right) & =\frac{d^{2} u^{a}\left(x_{0}\right)}{d x^{2}} \\
& \Downarrow \\
u^{a}\left(x_{0}\right) & =\sum_{I}^{N P} \frac{d^{2} N\left(x_{0}\right)}{d x^{2}} u\left(x_{I}\right) .
\end{aligned}
$$

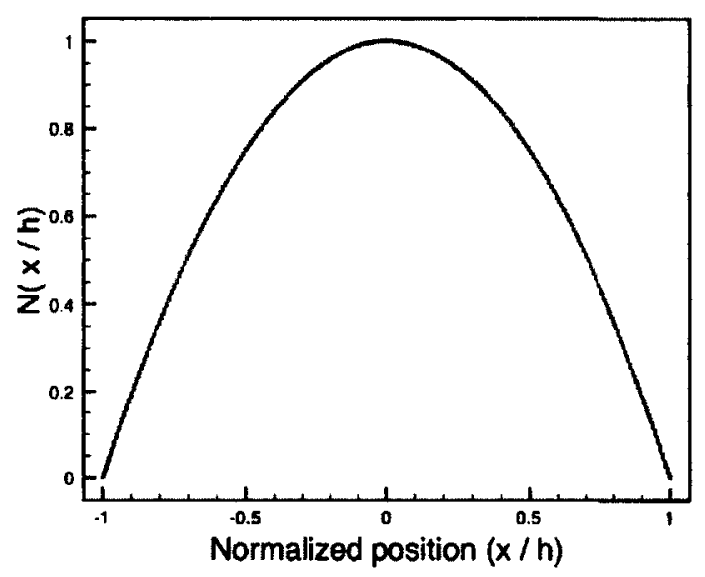

Figure 5: An example parabolic shape function.

To determine a full solution to the problem for all $x$ the function $u(x)$ becomes the vector $\mathrm{U}$ and the function $N(x)$ becomes the matrix $\mathbf{N}$ as in

$$
\begin{aligned}
u^{a} & =\frac{d^{2} u^{a}}{d x^{2}} \\
\Downarrow & \\
\mathbf{U} & =\mathbf{N}_{\mathbf{x} \mathbf{x}} \mathbf{U},
\end{aligned}
$$

with $\mathbf{N}_{\mathbf{x x}}$ being the matrix form of the second derivative operator of $\mathbf{N}$.

A problem can occur in this process if the points are not equally spaced. For example, using a self replicating function to determine the value at $u\left(x_{0}\right)$ one would want $N\left(x_{0}\right)=1$ and $N\left(x_{-1}\right)=N\left(x_{1}\right)=0$. If the point at $x_{1}$ is closer to $x_{0}$ than the 
typical node spacing then the value $N\left(x_{1}\right)$ will be nonzero and the summation will give an erroneous value for $u\left(x_{0}\right)$ from Eq. (3). This is considered an inconsistency as the shape function is not able to reproduce a known function exactly. Further additions to the creation of the shape function will involve enforcing consistency conditions, particularly the reproducing consistency conditions. These require that the shape function can accurately reproduce a given basis function, and their enforcement will be discussed later.

The two main shape function formulations that will be discussed for meshless methods are smoothed particle hydrodynamics (SPH) and moving least squares (MLS). SPH is considered the first meshless method to be developed and has a kernel or weighting function as its basis. MLS builds upon this by adding correction function coefficients and consistency conditions.

\subsubsection{Smoothed particle hydrodynamics}

Smoothed particle hydrodynamics, created to model astrophysical fluid flows, divides the computational domain into points which are described as particles. These points have a certain distance $h$ between them, a smoothing length, over which their properties are smoothed by a kernel weighting function similar to the basic shape function $N$ seen previously. Since this shape function does not involve a correction or consistency condition and simply weights the nodes it will be referred to as a kernel or weighting function.

The continuous form of the SPH,

$$
u^{a}(x)=\int_{\Omega} w((x-y) / h) u(y) d y
$$

is very similar to the discrete form shown in Eq. (3) with $w(x)$ the weighting function. 
The discrete form of SPH is:

$$
u^{a}(x)=\sum_{I}^{N P} w\left(x-x_{I}\right) u\left(x_{I}\right) \Delta V_{x_{I}}
$$

Both forms include a summation over the entire domain, $\Omega$, and all $N P$ nodes, however the weighting function quickly approaches zero as the distance from the node at $x$ increases.

For the method to produce accurate or stable results there are several conditions which the kernel weighting function, $w\left(x-x_{I}\right)$, are required to satisfy. A positivity condition ensures that for all values $x$ within the computational domain, $w(x) \geq 0$. The function must exclude points far from the node of interest, giving them zero weighting. The region of nonzero weighting is considered the domain of influence, or in our terms, the finite cloud. The weighting function must also conform to a normality property which helps ensure that there is no gain or loss in a system, similar to the consistency condition. This dictates that the integral of the function over the entire domain, or sum over all discrete points, must result in a value of unity.

As nearby points would be expected to have more of an influence than points further away, the weighting function must be monotonically decreasing with distance from the origin. Lastly, as the points become closer to each other, or $h \rightarrow 0$, the weighting function must act more like the delta distribution, $\delta(s)$. These conditions 
can be listed as:

1. $w(x-y, h)>0$ in the domain of influence $\Omega_{I}$, subdomain of $\Omega$,

2. $w(x-y, h)=0$ outside of the domain of influence $\Omega_{I}$,

3. a normality property: $\int_{\Omega} w(x-y, h) d \Omega=1$,

4. $w(s, h)$ is a monotonically decreasing function, where $s=\|x-y\|$,

5. $w(s, h) \rightarrow \delta(s)$ as $h \rightarrow 0$, where $\delta(s)$ is the Dirac delta distribution.

The nodes inside of the domain of influence for a particular node can also be termed its support domain or as mentioned, its cloud. The support domain is often a circle or sphere but can be square or rectangular and does not need to be symmetric.

There are several commonly used weighting functions which conform to these conditions such as an exponential, cubic spline or quartic spline. An example of a simple kernel weighting function is shown in Fig. 6.

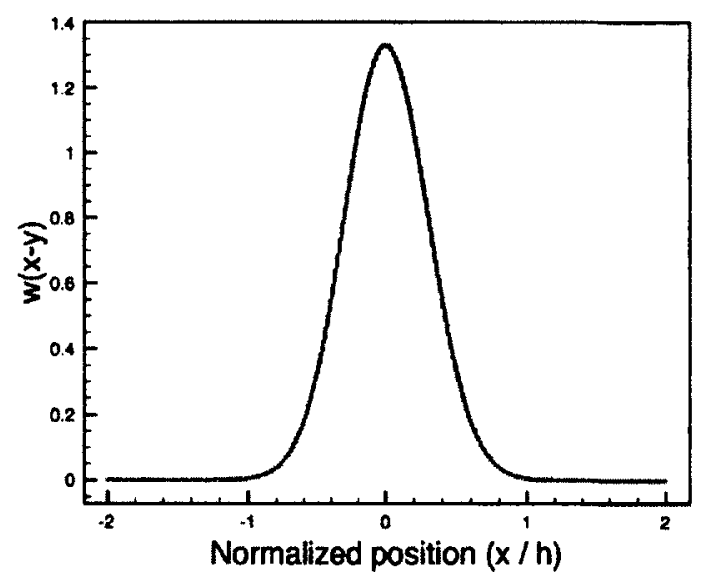

Figure 6: An example kernel function using an exponential weighting, both axes are unitless. 


\subsubsection{Moving least squares and kernel methods}

To improve on the accuracy of SPH, and to help solve the consistency problem, a correction function can be added. The coefficients for the correction function must be determined, using the consistency conditions, and can be found using a least squares approach. This type of solution is referred to as the moving least squares method. The discrete form of the method is shown below:

$$
u^{h}(\boldsymbol{x})=\sum_{i=1}^{m} p_{i}(\boldsymbol{x}) a_{i}(\boldsymbol{x}) \equiv \boldsymbol{p}^{T}(\boldsymbol{x}) \boldsymbol{a}(\boldsymbol{x})
$$

with $m$ being the number of terms in the basis function, $p_{i}(\boldsymbol{x})$, which is commonly a linear or quadratic basis and is further explained in Chapter 3 on the finite cloud method.

The MLS method provides a procedure for determining the correction coefficients of $a_{i}(x)$ by minimizing the function

$$
J=\sum_{I} w\left(\boldsymbol{x}-\boldsymbol{x}_{I}\right)\left[\sum_{i} p_{i}\left(\boldsymbol{x}_{I}\right) a_{i}(\boldsymbol{x})-u_{I}\right]^{2},
$$

the difference between the local approximation and the function, using a weighted least squares fit.

The minimum of $J$ is found by taking its derivative with respect to $a(x)$ and setting to zero, giving a matrix equation which can be solved to determine the coefficient values. The resulting MLS shape function,

$$
N(\boldsymbol{x})=\boldsymbol{p}^{T} \boldsymbol{a}(\boldsymbol{x}),
$$

is functionally equivalent to the base shaping function used in this thesis. A more 
thorough explanation and justification coming from a slightly different perspective is given in the following chapter, on the Finite Cloud Method (FCM).

As mentioned above, the unknown coefficients $a_{i}$ can also be determined by enforcing consistency conditions using a reproducing kernel or weighting function which will be further explored in Chapter 3 on the finite cloud method. These methods use a different procedure and justifications however the result is equivalent with the MLS method.

The remaining meshfree methods use very similar formulations with slight variations to obtain improvements in stability and consistency. Basis functions can be tailored to the desired type of solution, can involve a larger basis or enhancement functions such as $\sinh (n x)$, or $\cosh (n x)$ may be added. Lastly, kernel functions can be modified or be made to behave as more, or less, of a delta distribution, altering the weighting of nearby nodes in the shape creation. 


\section{Chapter 3}

\section{Finite cloud method}

The following chapter describes the Finite Cloud Method (FCM) and its implementation to solve various partial differential equations, and is based upon methods described in [14].

\subsection{Formulation}

The formulation of an FCM solution requires initially a domain of interest with enough points or nodes spread throughout to accurately represent the region and the desired function. For instance in one dimension and solving a wave type equation one would typically want a point density with at least 10 points per wavelength.

These nodes can be arranged in a regular or irregular distribution, and the placements can be easily tailored to suit the given problem. As well, each node has a support domain of the nodes surrounding it, a 'cloud'. This cloud of nodes is what will give rise to the shape function for the node of interest. To illustrate, Fig. 7 has an irregular distribution of nodes in two dimensions, along with example clouds for two of the nodes. The clouds can have varying shapes, as seen with a square or a circle, and it is not necessary for them to be of the same size, nor be perfectly circular 
or square. With this domain representation in mind, the following chapter covers the mathematical formulations and justifications for the FCM.

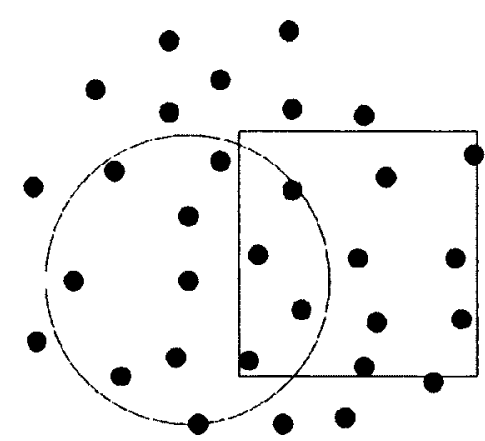

Figure 7: An example set of nodes in two dimensions and an irregular distribution. Also shown are the clouds for two nodes of interest. The clouds can be of different shapes and sizes depending on the local node distribution.

The FCM is a meshless technique applied to approximate the solution of actual functions using the fixed reproducing kernel technique [14]. A one dimensional kernel is used to illustrate the method however simple modifications allow for two or three dimensions. To begin, the function $u(x)$ is to be approximated and this approximation will be labelled as $u^{a}$. The form of the approximation is

$$
u^{a}(x)=\int_{\Omega} \zeta(x, s) \varphi\left(x_{K}-s\right) u(s) d s
$$

with, $\zeta(x, s)$ is the correction function and $\varphi\left(x_{K}-s\right)$ the kernel function centred at a point $x_{K}$, and again $u(s)$ being the function to be approximated. This form is similar to those shown previously in Chapter 2 .

The correction function,

$$
\zeta(x, s)=P^{T}(s) C(x)
$$


is comprised of two functions, a vector basis function $P(s)$ and the vector of correction function coefficients, $C(x)$. The vector basis function used is a quadratic basis, meaning that each nodal shape function is approximated with a quadratic function, $a+b x+c x^{2}$, and the coefficients must be found. Thus, any function $u$ which is second order or lower can be solved exactly. In one dimension a quadratic has three terms, thus the $P(x-s)$ vector is sized $3 \times 1$, but written $m \times 1, m=3$. The correction function $C(x)$ is also sized $m \times 1$. Since these are the coefficients for a quadratic basis and they must be solved by a system of equations, for a solution to be possible it is necessary to have a minimum $m$ points in a cloud to determine the $m$ coefficients. For two dimensions a quadratic basis is formed with $m=6$, and in three dimensions $m=10$. The bases are

$$
\begin{aligned}
P^{T}(s) & =\left[1, s, s^{2}\right], \quad m=3 \\
P^{T}(s, t) & =\left[1, s, t, s^{2}, s t, t^{2}\right], \quad m=6 \\
P^{T}(s, t, v) & =\left[1, s, t, v, s t, s v, t v, s^{2}, t^{2}, v^{2}\right], \quad m=10 .
\end{aligned}
$$

Expanding the original form from Eq. (13) we have

$$
u^{a}(x)=\int_{\Omega} P^{T}(s) C(x) \varphi\left(x_{K}-s\right) u(s) d s
$$

and coefficients for the correction function must now be determined. These coefficients are determined using a self reproducing consistency condition. Since the approximation has a quadratic basis, it must be able to exactly reproduce its own quadratic basis as

$$
p_{i}(x)=\int_{\Omega} P^{T}(s) C(x) \varphi\left(x_{K}-s\right) p_{i}(s) d s, \quad i=1, \ldots, m
$$


in the integral form. This can be modified to be discrete as

$$
p_{i}(x)=\sum_{I=1}^{N P} P^{T}\left(x_{I}\right) C(x) \varphi\left(x_{K}-x_{I}\right) p_{i}\left(x_{I}\right) \Delta V_{I}, \quad i=1, \ldots, m
$$

and the correction function coefficients can be solved. The remaining solution method involves rearranging the discrete form into matrices for each nodal cloud which can be solved with matrix decomposition. Since the above Eq. (18) must be solved for each $i=1 \ldots m$, this can be made into a system of equations in matrix form as

$$
\mathbf{M} C(x)=P(x)
$$

combining several equations into $\mathbf{M}$ which is called the moment matrix. Each cell, $M_{i, j}, 1 \leq i \leq m, 1 \leq j \leq m$, of the moment matrix is created as

$$
M_{i j}=\sum_{I=1}^{N P} p_{i}\left(x_{I}\right) \varphi\left(x_{K}-x_{I}\right) p_{j}\left(x_{I}\right) \Delta V_{I}
$$

keeping in mind that although the summation runs through all $N P$ points, any point outside of the cloud is weighted as zero thus reducing the necessary computations. The moment matrix has size $m \times m$ and is created as

$$
\mathbf{M}=\mathbf{F W} \mathbf{F}^{\mathbf{T}}
$$


with $\mathbf{F}$ being

$$
\mathbf{F}=\left[\begin{array}{cccc}
p_{1}\left(x_{1}\right) & p_{1}\left(x_{2}\right) & \ldots & p_{1}\left(x_{N P}\right) \\
p_{2}\left(x_{1}\right) & p_{2}\left(x_{2}\right) & \ldots & p_{2}\left(x_{N P}\right) \\
\vdots & \vdots & \ddots & \vdots \\
p_{m}\left(x_{1}\right) & p_{m}\left(x_{2}\right) & \ldots & p_{m}\left(x_{N P}\right)
\end{array}\right]
$$

and $\mathbf{W}$

$$
\mathbf{W}=\left[\begin{array}{cccc}
\varphi\left(x_{K}-x_{1}\right) \Delta V_{1} & 0 & \ldots & 0 \\
0 & \varphi\left(x_{K}-x_{2}\right) \Delta V_{2} & \ldots & 0 \\
\vdots & \vdots & \ddots & \vdots \\
0 & 0 & \ldots & \varphi\left(x_{K}-x_{N P}\right) \Delta V_{N P}
\end{array}\right]
$$

Once the moment matrix has been created for a specified node and cloud, the correction function coefficients are solved using

$$
C(x)=\mathbf{M}^{-1} P(x)
$$

With the correction function being determined, the original approximation equation, Eq. (18), can be found using

$$
u^{a}(x)=\sum_{I=1}^{N P} N_{I}(x) u_{I}
$$


With $N_{I}(x)$ being the final shaping or interpolation function operating on the unknown function $u_{I}$ at node $I$. The shaping function is defined as

$$
N_{I}(x)=P^{T}(x) \mathbf{M}^{-1} P\left(x_{I}\right) \varphi\left(x_{K}-x_{I}\right) \Delta V_{I}
$$

\subsubsection{Derivatives of the shape function}

For the shape function $\mathbf{N}$ to be of use it is necessary to find its derivatives such that it can behave as an operator. Looking back at the definition of the moment matrix, Eq. (20), it is possible to see that it is not a function of $x$, making an easier task of taking a derivative of the shape function. The sole dependency on $x$ in the shape function is the first quadratic basis function, $P^{T}(x)$, giving the derivatives of the entire matrix, $\mathbf{M}$

$$
\begin{aligned}
N_{I, x}(x) & =\left[\begin{array}{lll}
0 & 1 & 2 x
\end{array}\right] \mathbf{M}^{-1} P\left(x_{I}\right) \varphi\left(x_{K}-x_{I}\right) \Delta V_{I} \\
N_{I, x x}(x) & =\left[\begin{array}{lll}
0 & 0 & 2
\end{array}\right] \mathbf{M}^{-1} P\left(x_{I}\right) \varphi\left(x_{K}-x_{I}\right) \Delta V_{I}
\end{aligned}
$$

with $N_{I, x}(x)$ being the first derivative of the shape function for node $I$ with respect to $x$, and the $N_{I, x x}$ being the second derivative. Finally, using the entire shaping matrix, $N$, one can now approximate functions using appropriate derivatives and boundary values for entire domains by

$$
\begin{aligned}
u^{a}(x) & =N(x) u(x)=\mathbf{N U} \\
\frac{d u^{a}(x)}{d x} & =N_{x}(x) u(x)=\mathbf{N}_{\mathbf{x}} \mathbf{U} \\
\frac{d^{2} u^{a}(x)}{d x^{2}} & =N_{x x}(x) u(x)=\mathbf{N}_{\mathbf{x x}} \mathbf{U}
\end{aligned}
$$




\subsubsection{Kernel function \& kernel fixing}

The kernel function, represented as $\phi\left(x_{K}-s\right)$ is a weighting function for the points in the current cloud. As previously mentioned, there are restrictions on the kernel function that it must weight points close to the centre, $\left(x_{K}\right)$, with values approaching unity, and drop to zero outside of the cloud. The number of points per cloud must be at least $m$, as the solution requires solving for $m$ coefficients and thus requires at least that many lines in the system of equations. The kernel function used in this thesis is [15]

$$
\varphi\left(x_{K}-s\right)=1.2615 \times e^{-\frac{\left(x_{K}-s\right)^{2}}{0.15 \Delta x^{2}}} .
$$

with an average point distribution for the current cloud, $\Delta x$, and the kernel being centred at point $x_{K}$. Both the multiplication factor, 1.2615 and the divisor in the exponential, 0.15, were taken from the thesis on which the initial FCM work for this thesis was based. The multiplication factor is applied evenly to every point in a cloud and therefore has no effect on the final weighting and cloud representation. The divisor in the exponential affects the size of a finite cloud and the weighting of points at various distances. Since the clouds become scaled to a predetermined distance, as discussed in Chapter 4.1.2, the effect of the parameter on solution accuracy is reduced. The kernel function centred at $x_{K}$ can be used to calculate the shape functions for any of the nonzero points in its cloud. Since the cloud encompasses many points, one could create a shape function for node $x_{I}$ using a kernel function centred at any number of places, which would allow for multiple different shape functions for the given node. To prevent this multivalued problem the kernel is fixed at the location of the node of interest, thus each nodal shape function has a kernel fixed at its location. This has been shown in [14] to provide a single and consistent interpolation function for the entire domain. 


\subsection{Previous FCM work}

The first paper to describe the FCM was published by Aluru and Li in 2001. This paper described the method and presented several studies of the convergence and accuracy of the method for a variety of equations in one and two dimensions [14]. The paper initially tested Poisson and Laplace equations, but focused mostly on material equations such as heat conduction, elasticity, coupled thermoelasticity, stokes flow and piezoelectricity. Subsequent papers discussed how to improve the accuracy of the shape function, giving a list of positivity conditions for the matrix and methods to ensure these conditions [24].

The original authors of the FCM have since adapted the method to form a combined BEM and FCM approach in stress and strain problems [25]. This combined approach was then modified with a novel BEM and a bimaterial system discretized and solved using the one dimensional FCM [26]. Boundary conditions between the two materials in a one dimensional stretching problem are simplified as the continuity only requires that an interface node have the same amount of stretch approaching from either side, making the point single valued, which is a rather simple condition computationally. The situation is also made less complicated as the domain equations are treated as a materially homogeneous with only a change in variable value between the materials. Typically one would need the material derivatives or a materially inhomogeneous equation for this situation. Such approximations are tolerated due to the convenience of the interface conditions and the simplicity of the one dimensional case.

A combined approach has also been used to solve beam deflection and electrostatics in MEMS structures. The boundary cloud method (BCM) solved the electrostatics 
equations and was combined with the FCM which was used to solve for beam deflection [27].

More recently, in 2007, the FCM was adapted to solve Schrodinger's equation for potential wells in up to three dimensions, also in a materially homogeneous domain [15].

All past examples of the FCM seen by this author have been materially homogeneous solutions, or treated as such, and all have used simple Neumann or Dirichlet boundary conditions. As well, I have not seen the FCM applied to any three dimensional full vector type problems.

\subsection{Diffusion example}

An example of the process used to solve a PDE with the FCM is shown here using first a time independent solution. Several simplifications will be made for this example which are not necessary for the method but will be helpful in the explanation. The PDE to be solved is

$$
\frac{\partial^{2} u(x, t)}{\partial x^{2}}=\frac{\partial u(x, t)}{\partial t}+B
$$

which is a simplified diffusion equation, having a forcing function $B$ which can add sources or set boundaries when using Dirichlet or Neumann conditions.

The solution will be assumed to be at steady state, with $\frac{\partial u(x, t)}{\partial t}=0$, giving

$$
\frac{\partial^{2} u(x, t)}{\partial x^{2}}=-B
$$

as the governing equation or in matrix form

$$
\mathbf{N}_{\mathbf{x x}} \mathbf{U}=-\mathbf{B}
$$


This example will use one dimension and a total of 5 nodes labelled from $n_{1}$ to $n_{5}$ and will correspondingly have a shape matrix $\mathbf{N}$ of size $5 \times 5$. Dirichlet boundary conditions will be enforced on the exterior nodes with $U(1)=1.0$ and $U(5)=0$. This is accomplished using a forcing function $B(1)=1.0$ and $B(5)=0$, and a shape function with no x-derivative, $N(x)$. The remaining forcing function is set to zero with $\mathbf{B}$ as

$$
\mathbf{B}^{\mathbf{T}}=\left[\begin{array}{llllll}
1 & 0 & 0 & 0 & 0
\end{array}\right]
$$

Cloud size will be restricted to three nodes, thus each interior node will have two neighbours giving their relative positions to the central node as $[-1,0,1]$. Since the process is the same for all of the nodes, only the shape function for node $n_{2}$ will be shown.

We first begin by constructing the moment matrix for node $n_{2}$ as

$$
\mathbf{M}=\mathbf{F W F}^{\mathbf{T}}
$$

The one dimensional $\mathbf{F}$ matrix, having nodal positions in $x$ for nodes $\left[n_{1}, n_{2}, n_{3}\right]$ being $[1,2,3]$, using a quadratic basis will become

$$
\mathbf{F}=\left[\begin{array}{lll}
x_{1}^{0} & x_{2}^{0} & x_{3}^{0} \\
x_{1}^{1} & x_{2}^{1} & x_{3}^{1} \\
x_{1}^{2} & x_{2}^{2} & x_{3}^{2}
\end{array}\right]=\left[\begin{array}{lll}
1 & 1 & 1 \\
1 & 2 & 3 \\
1 & 4 & 9
\end{array}\right]
$$


and the corresponding $\mathbf{W}$ matrix with the cloud centred at $x_{K}=2$ is

$$
\mathbf{W}=\left[\begin{array}{ccc}
\varphi(-1) & 0 & 0 \\
0 & \varphi(0) & 0 \\
0 & 0 & \varphi(1)
\end{array}\right]=\left[\begin{array}{ccc}
0.001605 & 0 & 0 \\
0 & 1.2615 & 0 \\
0 & 0 & 0.001605
\end{array}\right]
$$

The moment matrix is then calculated as $\mathbf{M}=\mathbf{F W F}^{\mathbf{T}}$ resulting in

$$
\mathbf{M}=\left[\begin{array}{ccc}
1.2647 & 2.5294 & 5.0621 \\
2.5294 & 5.0621 & 10.1370 \\
5.0621 & 10.1370 & 20.3156
\end{array}\right]
$$

Since the diffusion equation is of second order, we are only concerned with the shape function on the second derivative, seen in the last line of Eq. (27). The resulting shape function for each of the three nodes the cloud becomes

$$
\begin{aligned}
& N_{I, x x}(1)=\left[\begin{array}{lll}
0 & 0 & 2
\end{array}\right] \mathbf{M}^{-1}\left[\begin{array}{lll}
1 & 1 & 1
\end{array}\right]^{T} \varphi(-1)=1.0 \\
& N_{I, x x}(2)=\left[\begin{array}{llll}
0 & 0 & 2
\end{array}\right] \mathbf{M}^{-1}\left[\begin{array}{lll}
1 & 2 & 4
\end{array}\right]^{T} \varphi(0)=-2.0 \\
& N_{I, x x}(3)=\left[\begin{array}{llll}
0 & 0 & 2
\end{array}\right] \mathbf{M}^{-1}\left[\begin{array}{lll}
1 & 3 & 9
\end{array}\right]^{T} \varphi(1)=1.0
\end{aligned}
$$

As we are using a regular distribution and have set all of the clouds for the nonboundary points to include the two nearest neighbours, the shape functions will be exactly the same for the three internal points and their calculations are not shown.

The shape function calculated for the edge nodes is the same process as above using $N_{I}$ instead of $N_{I, x x}$. This however does not need to be calculated as it is 
required to fulfill the reproducing conditions giving $N_{I}\left(x_{I}\right)=1$ with the remaining values of $N_{I}\left(x_{a}\right)=0, x_{a} \neq x_{I}$.

The entire shape matrix, identified by $\mathbf{N}$, can be seen below, showing only the nonzero entries

$$
\mathbf{N}=\left[\begin{array}{llllll}
1 & & & & \\
1 & -2 & 1 & & \\
& 1 & -2 & 1 & \\
& & & 1 & -2 & 1 \\
& & & & \\
& & & & 1
\end{array}\right]
$$

It should be noted that for this simple 1D case of uniformly distributed points the $N$ matrix is identical to that which would be obtained by the FDM. Solving the above diffusion equation, (30), with time independence gives

$$
\begin{aligned}
& \mathbf{N U}=-\mathbf{B} \\
& \mathbf{U}=-\mathbf{N}^{-1} \mathbf{B} \\
& \mathbf{U}=\left[\begin{array}{lllll}
1.00 & 0.75 & 0.50 & 0.25 & 0.00
\end{array}\right]^{T} .
\end{aligned}
$$

The given shape matrix, $\mathbf{N}$, could be used in the same manner in a time dependent example using an appropriate time stepping algorithm, such as backward Euler which will be explained in Chapter 5 , to give the solution at several different points in time as $\mathbf{U}$ approaches the steady state value. 


\section{Chapter 4}

\section{Improvements and initial tests}

This chapter details the improvements made during the creation and testing of the FCM PDE solving program. Following this, several simple test cases are presented to further demonstrate the method and ensure convergence of the solutions.

\subsection{Improvements in implementation}

As I have worked on the implementation and testing of the FCM several problems or irregularities in the solutions have been noticed. These problems have been studied and improvements to the method have been made and are outlined below. In addition an advantage of the method, solution interpolation, will be discussed.

\subsubsection{Modified FCM}

With the FCM fully implemented, as previously discussed, a variety of simple tests have been performed to assess the robustness of the method and to allow improvements in the implementation where possible. The first such test is a simple one 
Table 1: The error in the calculated first four eigenmodes for the simple eigenvalue test case.

\begin{tabular}{c|c} 
mode $(\mathrm{n})$ & Percent Error (\%) \\
\hline 1 & 0.004642175293523 \\
2 & 1.423806079850953 \\
3 & 1.715944978740423 \\
4 & 1.914637973405808 \\
\hline
\end{tabular}

dimensional eigenvalue case,

$$
\frac{d^{2} u}{d u^{2}}=\lambda_{n} u
$$

having eigenvalues $\lambda_{n}$ and with the boundary in the $\mathrm{x}$-direction $0<x<a$ having Dirichlet conditions: $u(0)=u(a)=0$. The solution of this simple case gives a set of eigenvectors of the form $\sin (n \pi x / a), n=1,2, \ldots$, and the eigenvalues being $\lambda_{n}=(n \pi x / a)^{2}$.

Knowing that the solutions are symmetric or anti-symmetric, a mirroring of the eigenmodes at the center of the model should result in the exact same eigenmodes, with a multiplication of -1 for the anti-symmetric modes. Symmetric modes about the origin have the relation $u(x)=u(-x)$, and anti-symmetric modes having $u(x)=$ $-u(-x)$

The test case has an upper boundary of $a=1000$ giving $0 \leq x \leq 1000$. The solution for the first four eigenmodes are shown in Figure 8(b) along with their reflections to verify symmetry of the solution. As can be seen there is a discrepancy in the solutions which breaks the symmetry. As well, knowing the eigenvalues, the errors on the first four eigenvalues can be calculated and are shown in Table 1.

To find the root of this problem it was necessary to look into the formulation of the shape function, in particular the moment matrix for each node in the model. The 


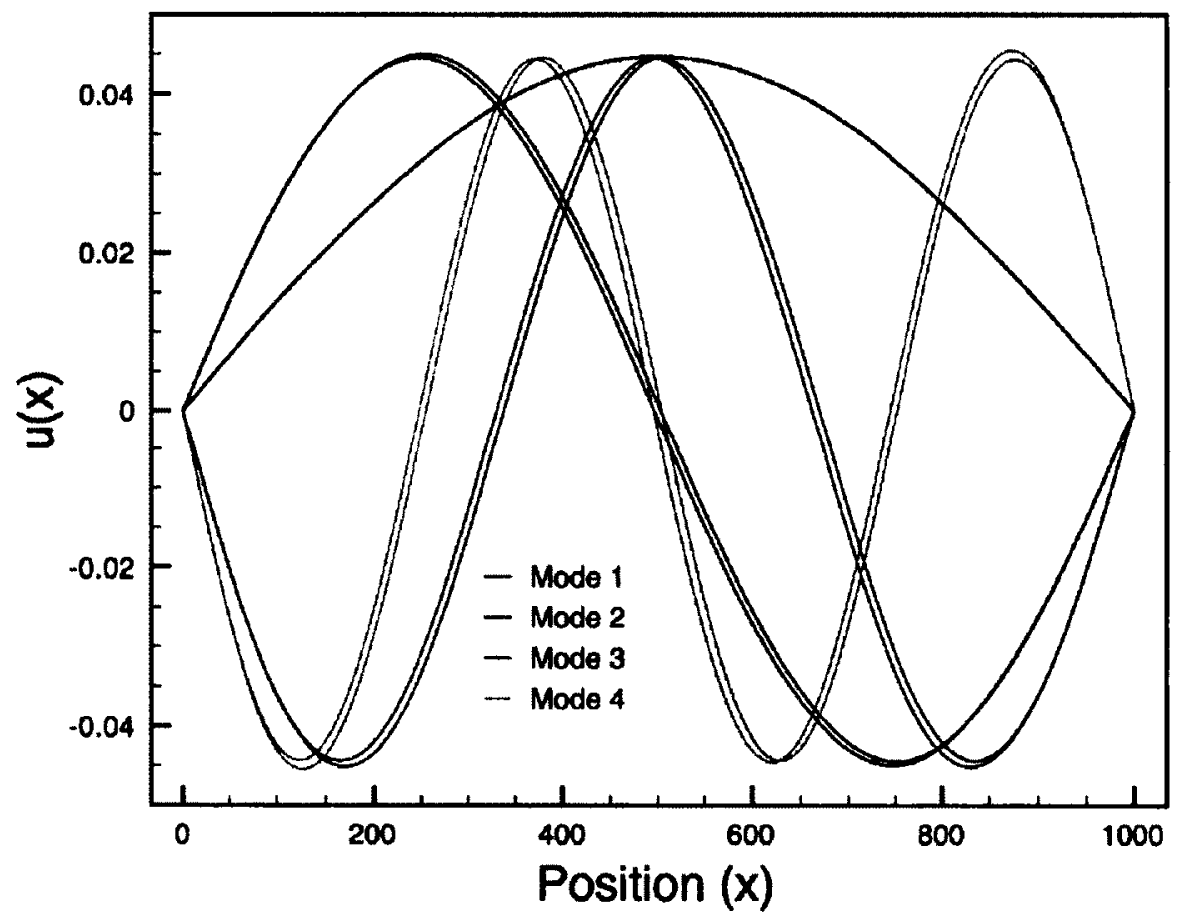

Figure 8: First four eigenmode solutions along with their mirrored counterparts for the Dirichlet eigenvalue test case. 
moment matrix is composed of two main parts: a matrix of quadratic basis functions $\mathbf{F}$, and a kernel weighting function matrix $\mathbf{W}$. It is then formed as $\mathbf{M}=\mathbf{F W F}^{\mathbf{T}}$, and an inversion or decomposition is used, as previously described, to determine a correction function. The ability to accurately perform a numerical decomposition and solution is related to the condition number of a matrix. The condition number gives an estimate on the inaccuracy of a numerical matrix solution or approximation. A large condition number is indicative of an ill-conditioned matrix, and the solution of such a system is much more likely to be in error [28]. A matrix with a large spread in the order of magnitude of its values will typically result in a poorly conditioned matrix.

The $\mathbf{F}$ matrix contains one column for each of the nodes in a given cloud. Each column $j$ of the matrix corresponds to one of $\left[1 x_{j} x_{j}^{2}\right]^{T}$. As the model starts at the origin, if the cloud extends to the node at $x=3$, the column for this node will be $\left[\begin{array}{ll}1 & 39\end{array}\right]^{T}$. A node at the origin will have a column in the $\mathbf{F}$ matrix $\left[\begin{array}{ll}1 & 0\end{array}\right]^{T}$. There is not a large difference in the order of values in $\mathbf{F}$ between the nodes at $x=0$ and $x=3$, with the values ranging from 0 to 9 .

A cloud located at the upper boundary of the model however, at $x=1000$ will have a quadratic basis of $[110001000000]^{T}$, giving a very large range in values for the $\mathbf{F}$ matrix. The kernel weighting values in $\mathbf{W}$ are based on the difference between the nodal positions and thus will not be affected by the cloud position.

One can calculate the condition number for the moment matrix for each cloud used along the entire domain. A plot of condition number as a function of cloud position is shown in Fig. 9, showing well conditioned values near the origin and quickly becoming poorly conditioned as the cloud moves to larger values in $x$.

With the error and the numerical reason for the error understood, it became possible to devise a solution. The quadratic basis functions can be created using a 


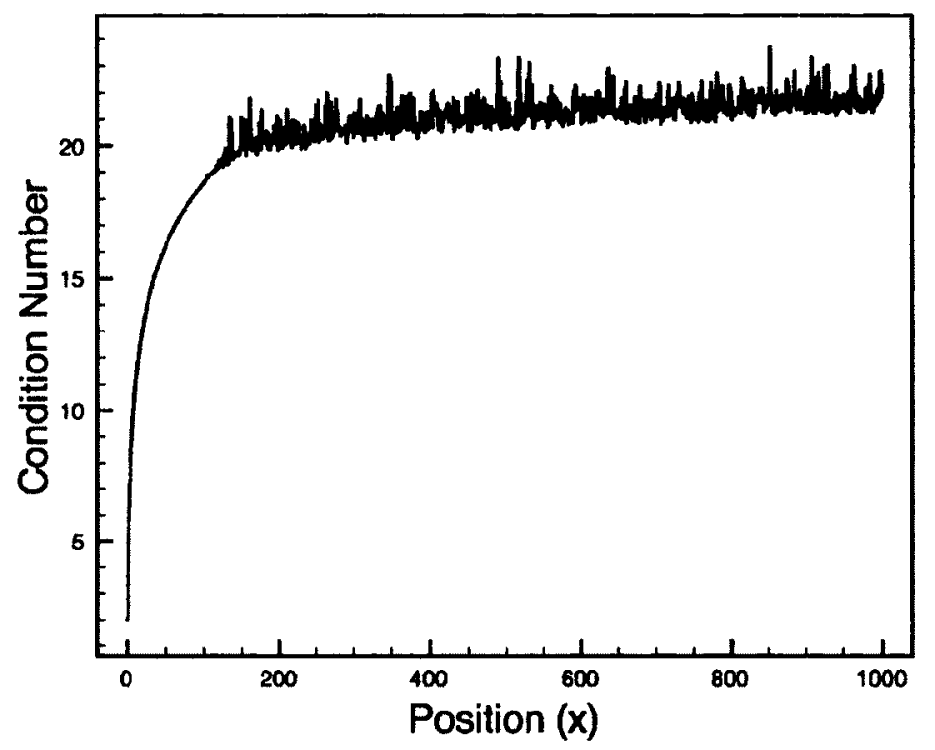

Figure 9: Condition number for the moment matrix for all clouds in domain $0<$ $x<1000$

relative difference between a node in question and the center of the cloud, instead of the absolute position of the nodes. This results in a translation of the nodes in question to the origin, keeping the position of the nodes relative to each other consistent.

This translation of points reduces the differences in order of magnitudes of the moment matrix, as well removes any numerical differences between regions far removed from each other. The result of this is an improvement, or lowering, of the condition number for the moment matrix and consistent numerical values regardless of the cloud location in the domain.

With the fix implemented and the above model tested, the improvements to the solution are quite clear. The condition number both before and after the fix are shown in Fig. 10(a), along with a plot of the solutions and their mirrored counterparts in Fig. 10(b). Lastly, Table 2 has the error in eigenvalues for the first four modes with and without the translational fix. 


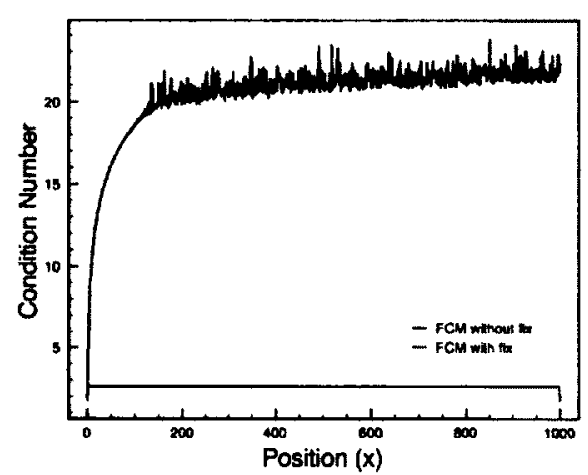

(a)

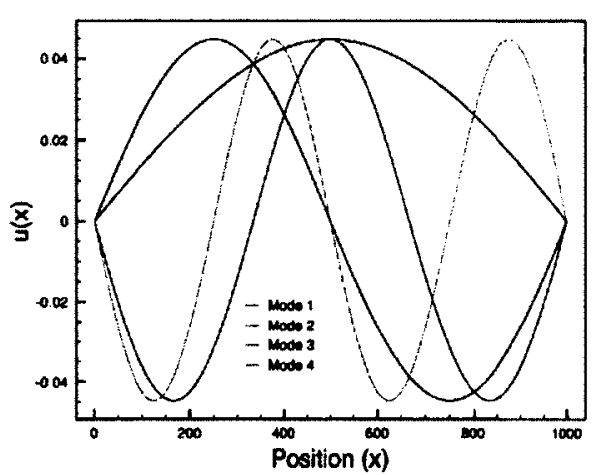

(b)

Figure 10: (a) Condition number for the moment matrix for all clouds in domain $0<x<1000$ with and without the translational fix. (b) First four eigenmode solutions along with their mirrored counterparts after the translational fix.

Table 2: The error in the calculated first four eigenmodes for the simple eigenvalue test case.

\begin{tabular}{c|c|c} 
mode (n) & Percent Error (\%) & Fixed-FCM Percent Error (\%) \\
\hline 1 & 0.00464 & -0.00008 \\
2 & 1.42381 & -0.00033 \\
3 & 1.71594 & -0.00074 \\
4 & 1.91464 & -0.00131 \\
\hline
\end{tabular}


This point translation of the system has a second beneficial result, which reduces the calculations required for the shape function and its derivatives. The examined node at $(x)$ will always be at the origin, the functions from (27) become

$$
\begin{aligned}
\left.N_{I}(x)\right|_{x=0} & =\left[\begin{array}{lll}
1 & 0 & 0
\end{array}\right] \mathrm{M}^{-1} P\left(x_{I}\right) \varphi\left(x_{K}-x_{I}\right) \Delta V_{I} \\
\left.N_{I, x}(x)\right|_{x=0} & =\left[\begin{array}{lll}
0 & 1 & 0
\end{array}\right] \mathbf{M}^{-1} P\left(x_{I}\right) \varphi\left(x_{K}-x_{I}\right) \Delta V_{I} \\
\left.N_{I, x x}(x)\right|_{x=0} & =\left[\begin{array}{lll}
0 & 0 & 2
\end{array}\right] \mathbf{M}^{-1} P\left(x_{I}\right) \varphi\left(x_{K}-x_{I}\right) \Delta V_{I} .
\end{aligned}
$$

\subsubsection{Node Scaling}

\subsubsection{Node scaling}

With the creation of more complicated domains and improved node mapping techniques, there will exist areas of high node density and areas of low node density. As has already been shown above, node placement and the distances between nodes can have an effect on the quality of the moment matrix as well as its condition number and thus the reliability of its decomposition.

We wish to minimize this condition number for every $\mathbf{M}$ matrix which will increase the numerical stability and accuracy of the solutions. A small routine has been created to mimic the creation of a cloud in two dimensions using nine points in a regular grid shape. The spacing, $d x$, between the grid points was then varied for a wide range of values. For each cloud a moment matrix was created and the condition number recorded.

The results from this test, Fig. 11, show that there is a minimum in the condition number around a spacing of $d x \approx 10$. From this test we can see that an ideal cloud will have an approximate spacing of 10 units between its constituent nodes and thus 
we wish to scale the clouds to obtain this relation.

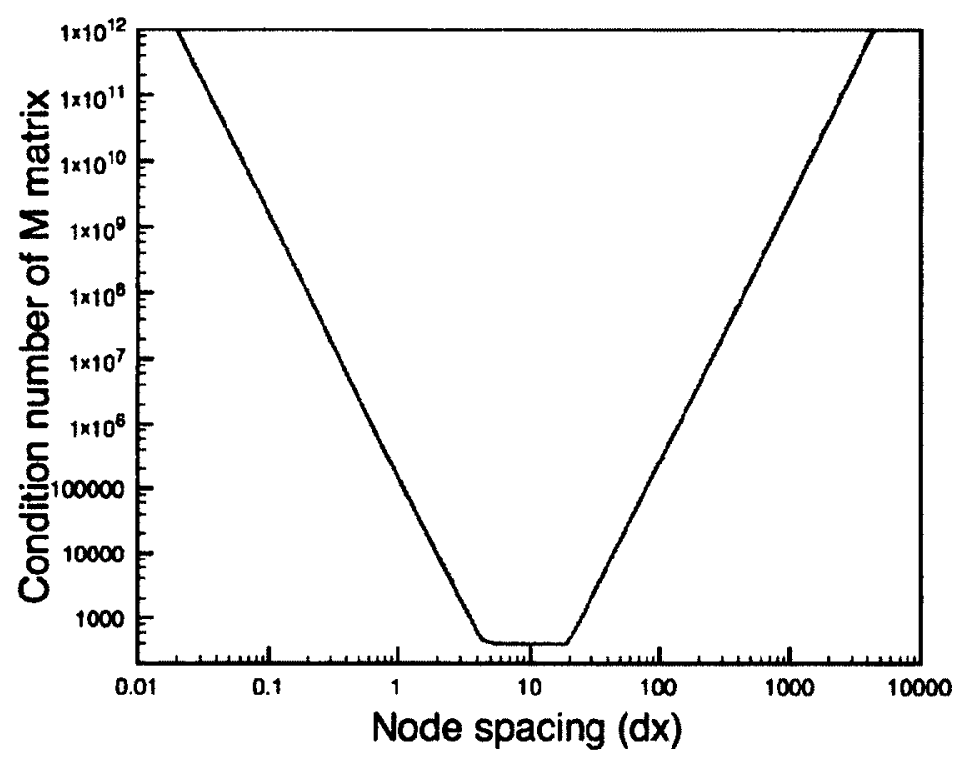

Figure 11: Condition number for the moment matrix for clouds with a varying $d x$ value

This scaling is accomplished in a several step process. First, the cloud for a node, $n_{I}$, is created and the minimum distance between $n_{I}$ and a node in the cloud is determined, $d x$. The nodal positions of the cloud are then multiplied by a scaling factor $d_{\text {scale }}$ to increase the minimum distance to be five units. This corresponds to the minimum $d x$ size at the lowest condition number while allowing for uneven node placement which may have larger spacing. The goal being to keep the scaled spacing values in the minimum of Fig. 11. The traditional shape function method can then be performed on the scaled cloud, while still using cloud translation from above.

Once the shape functions are created for the scaled cloud they must be descaled to return them to their proper units. The first derivatives, eg. $N_{I, x}$ and $N_{I, y}$, have units of (units $\left.\times d_{\text {scale }}\right)^{-1}$ and must be descaled using $N_{I, x} \times d_{\text {scale }}$ returning their units to $(\text { units })^{-1}$. Second derivatives, eg. $N_{l, x x}$, must be descaled by $\left(d_{s c a l e}\right)^{2}$ giving a final result of $(\text { units })^{-2}$. Further testing of the scaling using three dimensions or 
more points per cloud all gave the same minimum condition number as above at the same $d x$ distribution.

\subsubsection{Cloud fixing}

Following the node scaling, it was noticed that there were often errors in solutions at a transition from a high point density area to a low density area. The errors were noticed to be particularly harsh in three dimensional domains.

An examination of such a transition node showed that its cloud, or domain of influence, was highly lopsided. An example of a situation in two dimensions is shown in Fig. 12. One can see a point distribution with a low density area and an area with nine times the points per area. The colours of the points represents the condition number of the moment matrix for that particular node. With blue and purple being a very low, or good condition number, and moving towards red being a larger condition number approaching 2.0 in this case. Green nodes are points with a moment matrix condition number larger than 2.0. One of the clouds has a green box surrounding it showing its cloud. One can see that while searching for nearby nodes, a small increment outward in the $\mathrm{x}$-direction may add significantly more nodes on the positive ' $x$ ' side than on the negative ' $x$ ' side. This leads to an imbalanced cloud and a poor condition number. By identifying these nodes at runtime the cloud size can be altered or expanded in a certain direction to rebalance the cloud and improve the condition number of its moment matrix.

Thus as the shape matrix is being formed, in essence, the 'quality' of each nodes cloud can be evaluated and fixed to improve accuracy of the future solution. For this particular example an abrupt transition from high density to low density was chosen on purpose to demonstrate the problem. By setting a max condition number of 2 , the clouds can be fixed by increasing their size asymmetrically to balance their point 


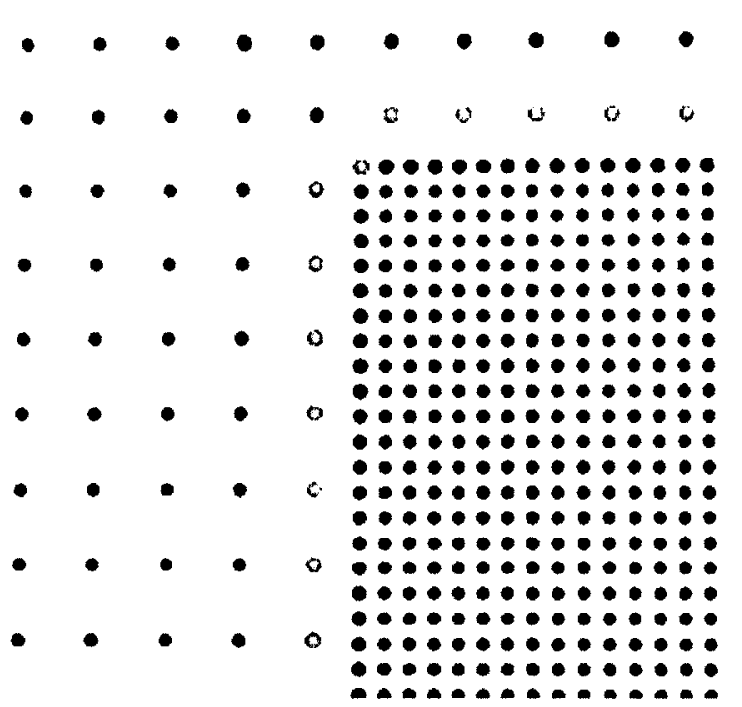

Figure 12: Point distribution for the cloud fixing example with an area of high point density directly touching an area of low density. Points are colour coded from blue to red for condition numbers 0 to 2.0 , points with a moment matrix condition number above 2.0 are shown in green. An initial unfixed cloud with a poor condition number is shown by the green box.

distribution. The example tested is a two dimensional poisson problem which will be further explored in Chapter 4.3.2. To measure the error, we use a global error measure [14],

$$
\epsilon=\frac{1}{\left|u^{(e)}\right|_{\max } \sqrt{\frac{1}{N P} \sum_{I=1}^{N P}\left[u_{I}^{(e)}-u_{I}^{(c)}\right]^{2}}}
$$

with $\epsilon$ as the error in the solution, and the exact and computed solution denoted by superscript (e) and (c) respectively. By adding the node fixing solution, the error was reduced from 5.76 to 0.0057 , a significant reduction.

As an example of condition number improvement, several moment matrices had initial numbers 2.02 , or 3.93 and they were reduced to 1.49 and 1.12 , respectively. Condition number related errors have been found to be even more troublesome in three dimensional problems making cloud fixing essential for accurate solutions. A 
typical maximum condition number for a cloud in a three dimensional problems is 6.0 .

\subsubsection{Solution interpolation}

A beneficial feature of the finite cloud method is that, as mentioned previously, during the creation of the shape matrix all of the derivatives at the nodes can be trivially calculated and stored. These derivatives are typically used in the solution process to find the values of the field variables at each node as in $\mathbf{N}_{\mathbf{x x}} \mathbf{U}=\mathbf{U}$.

This also allows the final solution points and the derivative matrix to be used along with a Taylor series expansion to interpolate for any given point in the domain. From this, once a solution has been determined it is possible to interpolate that solution to any point within the domain quickly and easily with the shape function data that has already been created during the solution process. The solution is found using a quadratic basis, hence a second order Taylor series expansion,

$$
f(x)=f(a)+\frac{f^{\prime}(a)}{1 !}(x-a)+\frac{f^{\prime \prime}(a)}{2 !}(x-a)^{2},
$$

which is used for the interpolation.

A point is first chosen for the location at which one wishes to determine the unknown field. This unknown field is found using the closest node to the point along with its field solution, and first and second order derivatives. Such a routine could be used after a solution to create a smooth and continuous function from a set of discrete points for the purpose of plotting. As well, if any extra points are to be added during a simulation, such as with adaptive mapping, the field variables can be quickly and easily calculated for the new points. In the following sections, any figure which shows continuous field values instead of a discrete set of values has used this interpolation 
method.

\subsubsection{Multi-threading}

The creation of the matrices required to solve a problem can be divided into three steps. The first step is the placement of points throughout the computational domain which is a very quick process as there are few rules or restrictions on their placement. The second part involves the creation of the shape functions for each point, this is the longest step in the matrix creation as it involves creating a local cloud, creating and inverting a moment matrix and the storing the shape information for every point. The last step is taking the shape information and properly forming the solution matrices for the particular differential equation. This last step is also very fast computationally as it only requires moving data or pointers, and occasionally some multiplication.

In order to make the entire process faster and more efficient focus is placed on the shape function creation. This step only depends on the location of the points, which have already been placed, and can thus be made to run in parallel using multiple CPU cores and multi threading. The ease of parallelization offers a unique advantage over the FEM which must follow geometrical rules to discretize the domain, being dependent on neighbouring shapes, and cannot be treated as fully independent, making parallelization much more difficult.

To add multi threading to the shape creation, first all of the points in the domain are evenly distributed amongst the desired number of threads. This number is typically the number of cores in the CPU multiplied by the number of threads each core is capable processing, and can be queried by a program at runtime.

Once the points have been evenly divided each thread simply creates the shape functions for the points that it has been assigned. After all threads have finished, the shape functions are combined into one master list. As the number of processors rises 
this multi threading can dramatically speed up the solution process.

To demonstrate, I have created a two dimensional model with 382,704 points. Using a single thread the shape matrix creation for this model on an Intel $2.6 \mathrm{GHz}$ processor takes 32 seconds. This particular processor is capable of handling 8 threads simultaneously (two for each of the four cores) and using all 8 threads the shape matrix creation takes 7 seconds, a speed improvement of more than a factor four. One may expect a factor 8 improvement when using 8 threads, however, there are still only four cores and the hyper threading does not give 8 truly independent parallel processes. As well, with more threads combined with a relatively short creation time, a larger percent of the time is spent splitting and then merging the finished data.

\subsection{Implementation}

The finite cloud method, as described above, along with a three dimensional geometry creation tool and various equation solving engines has been created in a ' $\mathrm{C}$ ' program. The heart of the code is the shape function creation using the finite cloud method, which can be easily tailored to the PDE of choice. The code is currently unavailable to the public, however, I plan to fully release the code under a GNU public license to encourage the further use and development of the program.

The approach to geometry creation is to treat every shape independently at first, and to add nodes throughout each shape. Different shapes are then pieced together, simply keeping the boundary nodes as junction nodes, and removing any nodes that are too close to one another. From this, complex geometries can be created using a basic set of shapes and no need to code complex mesh generating and matching algorithms.

This ability to join structures together is taken to its full advantage when paired 
with the Atar geometry creation tool. Atar is an advanced FD PDE solving tool created by Tom Smy of Carleton University [8]. This geometry creation tool uses advanced non uniform meshing and a quad tree mesh and can be easily used to create rectilinear geometries. Points are then placed at the center, and faces in some instances, of the FD elements and thus can be used with the FCM. Curvilinear shapes are created with the FCM geometry tool and similarly joined with the Atar created node distributions for a joint Atar-FCM point distribution.

\subsubsection{Basic geometry element creation}

A set of simple geometric shapes, and node mapping routines for the shapes, has been created in the FCM geometry creation tool. These shapes are then combined to define the objects within the computational domain. The geometric mapping routines have been created with a variety of customizable parameters which can be altered in order to obtain the desired node mapping and final shape.

To begin with, the standard three dimensional shapes created are quadrahedrals, arcs, cylinders and spheres. These can be seen in Fig. 13. In two dimensions we have squares, polygons, arcs and discs, as seen in Fig. 14.

There are also a number of meshing parameters with each shape to customize the boundaries and nodal distributions. Boundary distributions can have no extra points, a single line of extra points or a double line of extra points, seen in the arc in Fig. 15.

A nonuniform node density can also be created such as a linearly varying radial distribution, Fig. 16. Natural distributions such as radial for discs and circles, or cartesian for polygons are used. One can also use cartesian for circles, Fig. 17(a), cartesian with a slightly random distribution, Fig 17(b), or purely random distributions, Fig. 17(c). The symmetry of the shapes can also be altered. For a circle one 

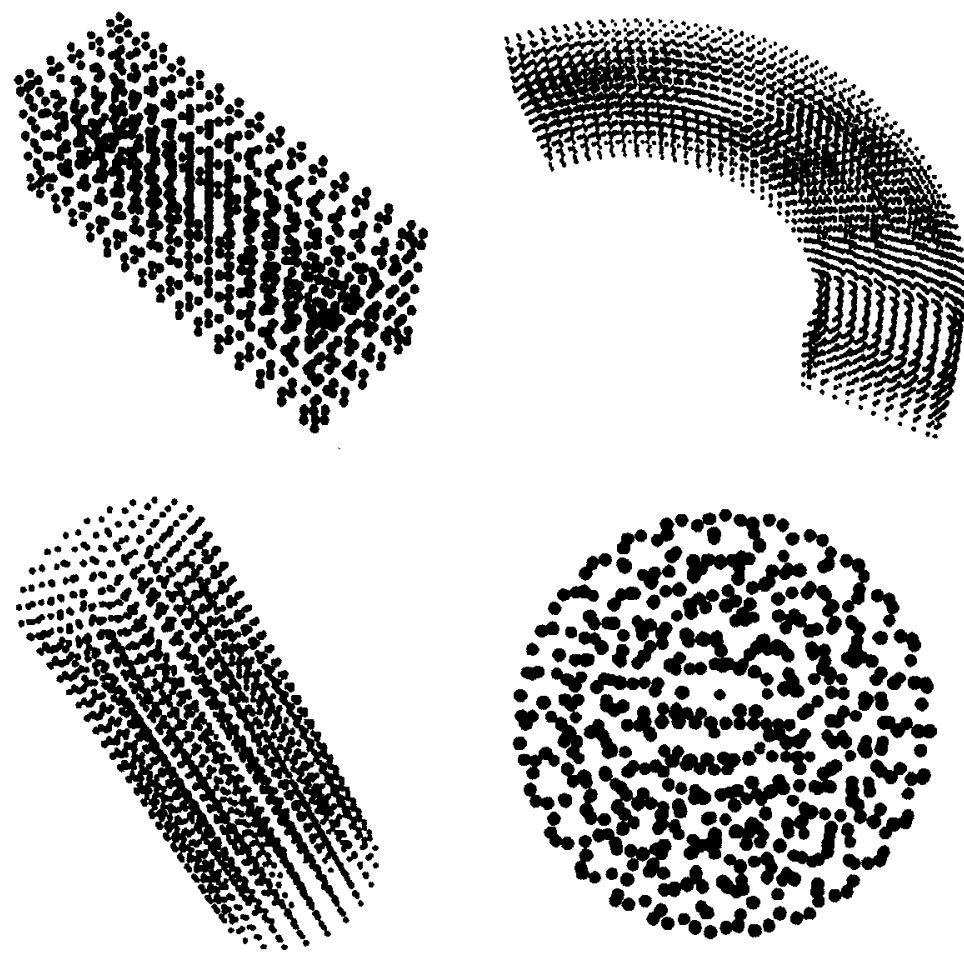

Figure 13: Example of the fundamental three dimensional shapes used in the FCM geometry creation engine. 

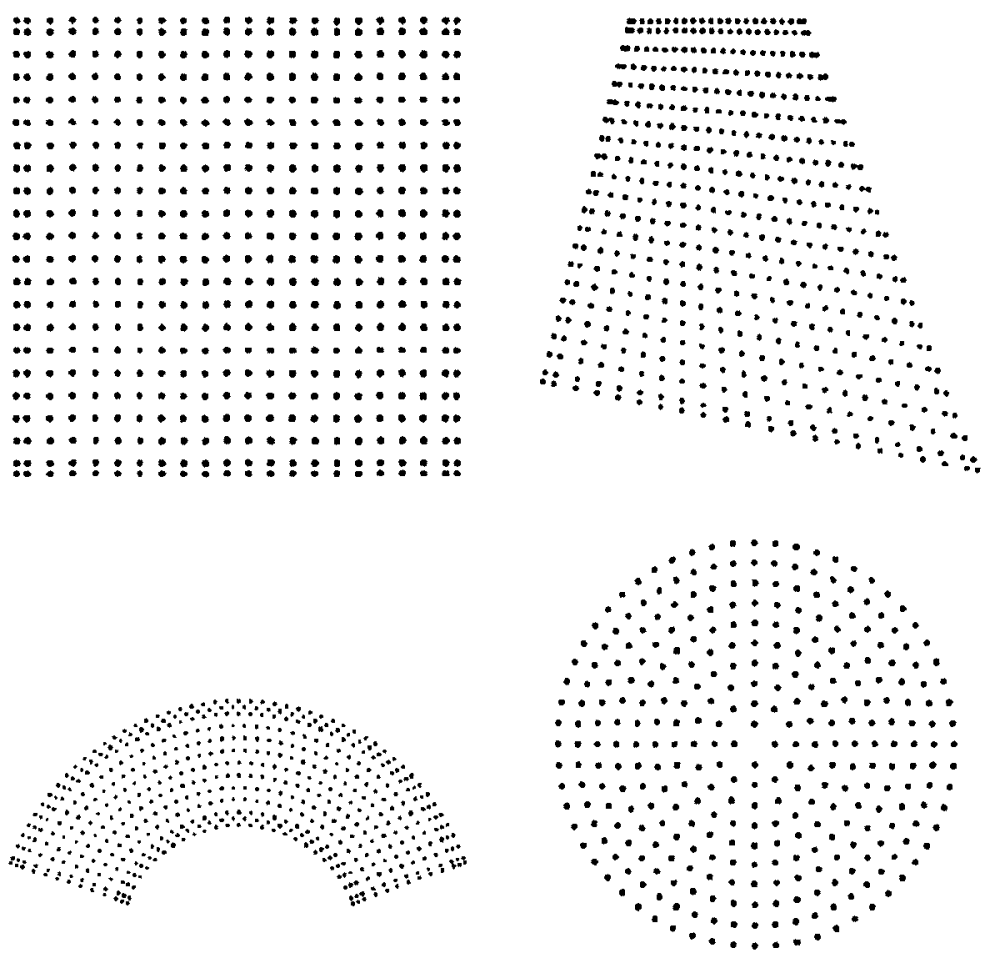

Figure 14: Example of the fundamental two dimensional shapes used in the FCM geometry creation engine.

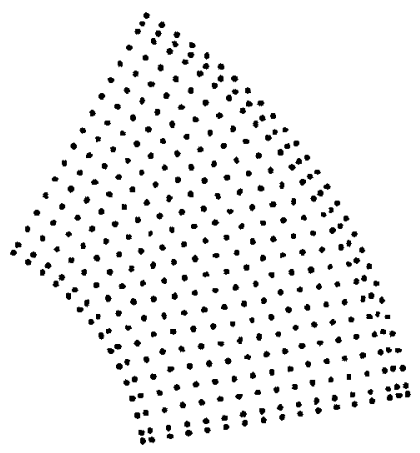

Figure 15: Example of an arc with different boundary node densities for the different sides, double row on the outer radius, single row on the inner radius and maximum phi angle and no extra points for the minimum phi angle boundary. 
can have, for example, fourfold symmetry about the center, or threefold symmetry, Fig. 18.

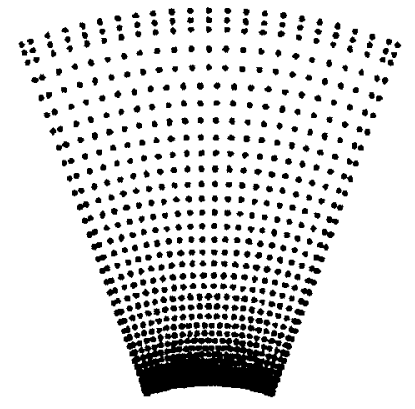

Figure 16: Example of an arc with a linearly varying density, from high density at the inner radius to lower density at the outer radius.

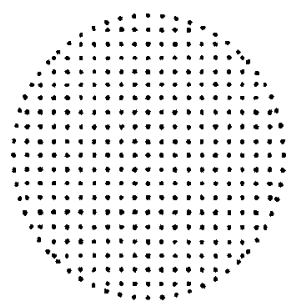

(a)

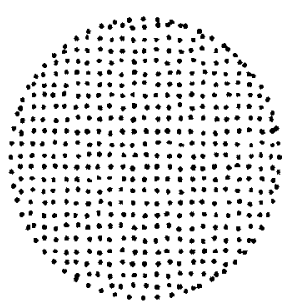

(b)

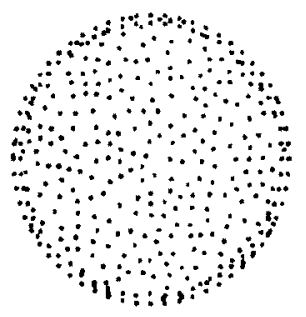

(c)

Figure 17: Example of the alternate inner shape point distribution options for a disc. (a) cartesian (b) cartesian with random 'jiggle' added (c) purely random with a minimum allowed distance between points.

Lastly, one can customize the boundary condition on any of the edges of a shape. This boundary condition can be seen in the varying colours of the boundary points in the polygon and arc, Fig. 19.

\subsubsection{Merging shapes}

The domain definitions are then created as a set of the above shapes which are merged together. Shapes are listed, along with their parameters in a node definition file, and 


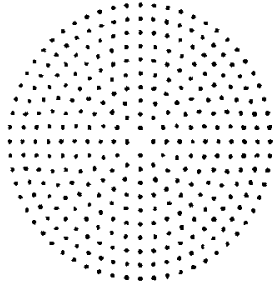

(a)

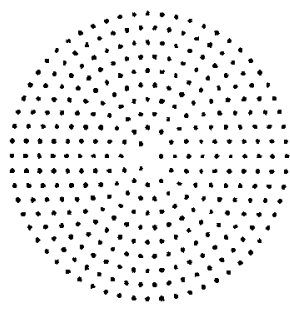

(b)

Figure 18: Example of two inner shape point symmetry options for a disc, (a) fourfold symmetry (b) threefold symmetry.

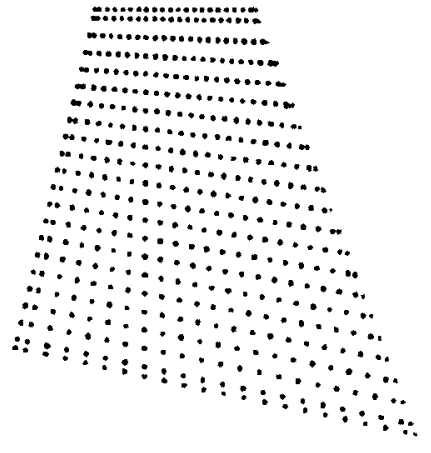

(a)

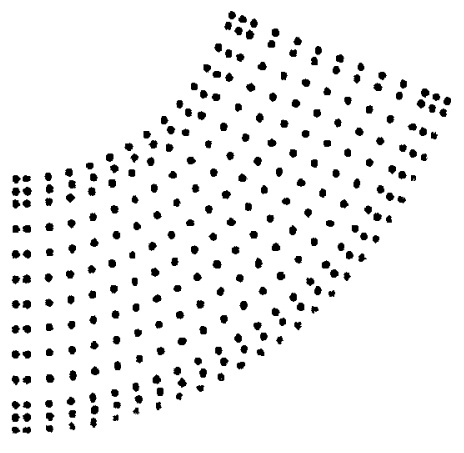

(b)

Figure 19: Example of (a) a polygon and (b) an arc with differing boundary conditions on each of its four edges. 
the shapes are created in the order in which they are listed. The process begins with the first shape listed in the file, and its nodes are mapped out as seen in Fig. 20. Also shown in the figure are the boundary normals which are stored for all boundary and interface points.

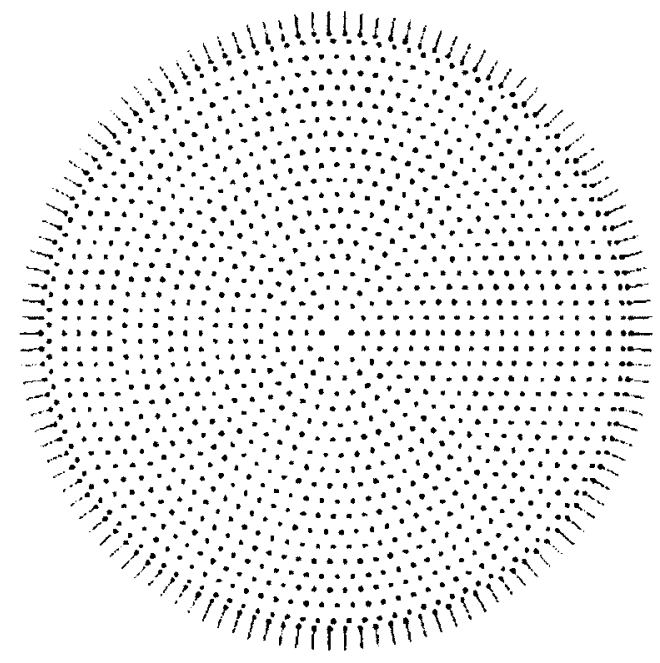

Figure 20: The first shape in a geometry mapping file with the boundary normals shown.

The next shape listed will typically begin by clearing out any previous nodes which lay within its boundaries along with any points that are within a specified distance of its boundaries, Fig. 21. Shapes can, however, be specified to not remove any points within their region and will then simply add to the points already created. Points that are kept, and are within the boundaries of the new shape, will be 'renamed' to belong to the current shape.

Next, points are added in the specified distribution to the interior of the new shape. Boundary nodes are added with the specified boundary density, as well as any outer 'padding' nodes which are also specified. Points which are on the interface between two shapes are defined as interface nodes, while points which are on an exterior are defined as boundary nodes, Fig. 22. This process is repeated for all remaining shapes, 


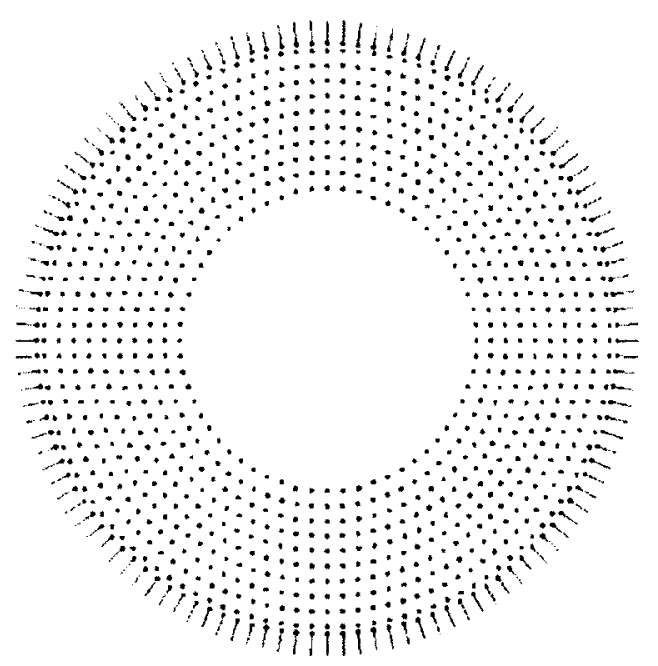

Figure 21: The second step in mapping out nodes, removing any points within the area of the second shape listed.

in their order of definition, and can be used to create rather intricate computational domains.

\subsubsection{Using Atar models for point generation}

As mentioned previously, the FCM has the ability to easily join point distributions which allows for the use of multiple point generating tools. The in house FDM tool Atar has an advanced geometry meshing engine which can generate shapes to be used as a basis for point distributions.

The meshing engine is a $3 \mathrm{D}$ tool used for planar rectilinear geometries which are defined by layer structure file. Meshing is done using an advanced quad-tree mesh for non-uniform block size refinement. Output from the program is a set of blocks with a defined geometry, position, and material which can then be read in using a conversion program, Atar-FCM.

As the blocks are read in, each material found is treated as its own region with its own subset of blocks and is treated independently until a later merging of regions. 


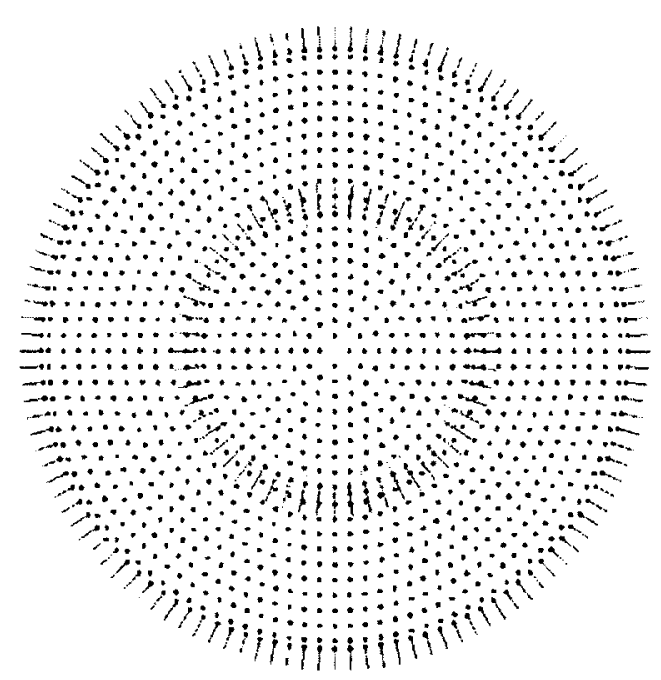

Figure 22: Example of the final node mappings for a geometry file containing two discs, showing the points coloured according to their associated material and the boundary and interface points as well as their normals.

An example of an Atar model with quad tree meshing is shown in Fig. 23.

To include the Atar block models in the FCM domain, a set of rules are needed to assign points to the blocks. The following list of rules for transferring blocks to points was attained after a number of iterations and refinements. These rules are divided between internal blocks, transitions in mesh densities, material interfaces, exterior boundaries and integrations with other shapes, regions and geometries.

\section{Atar rules}

\section{Internal Blocks}

1. Node at block centre.

\section{Mesh Transitions}

1. Node at average position on transition to smaller block of same material.

2. Mesh size transitions should have a depth of at least 2 Blocks. 


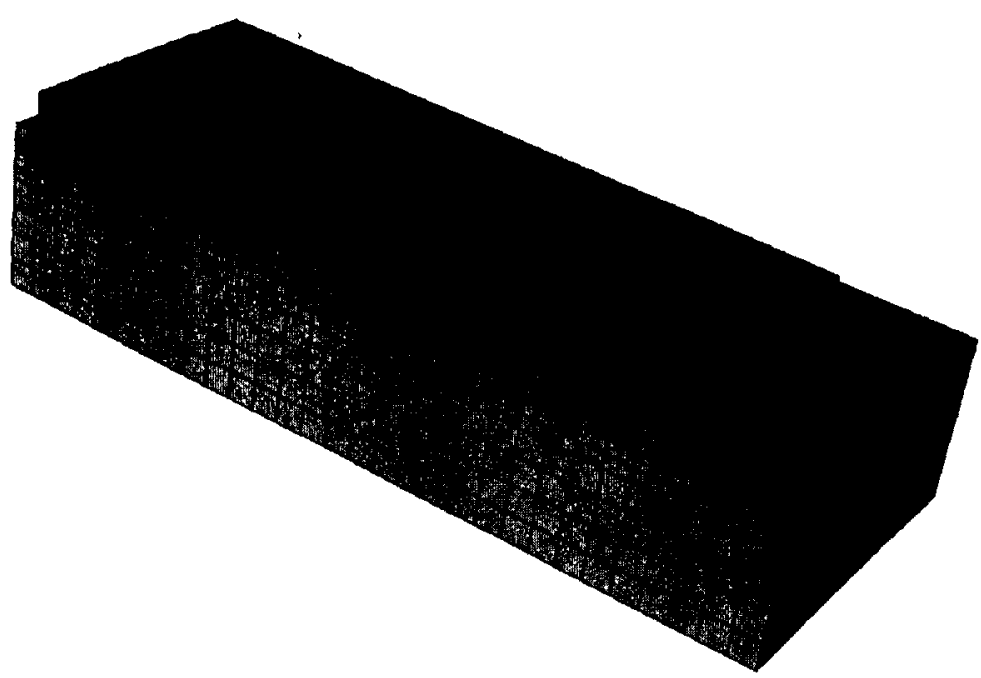

Figure 23: Example of an Atar model and quad-tree mesh.

\section{Material Interfaces}

1. Add nodes at center of block side.

2. Do not do a mesh transition across a material interface.

\section{Exposed Faces}

1. Add nodes at corners of exposed faces.

2. Mesas should have nodes at corners and as discussed in Chapter 5.5.2, should be weighted.

\section{Integration with other geometry elements}

1. Node densities at surface should be approximately equal.

2. No density transitions over the first few clouds. 


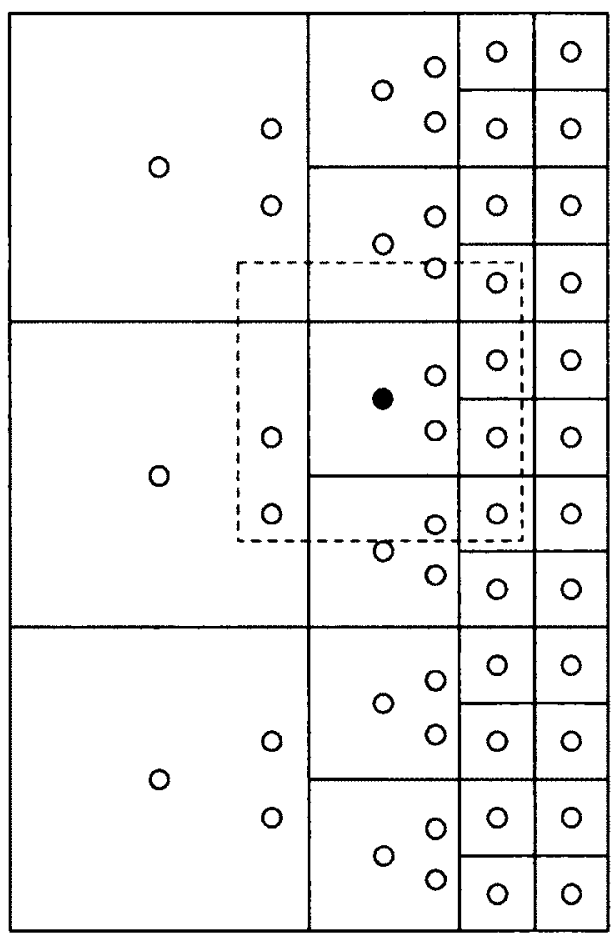

(a)

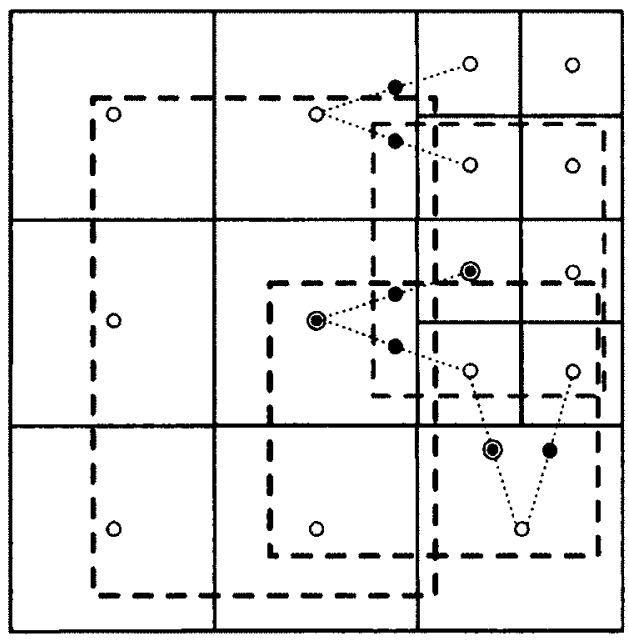

(b)

Figure 24: Atar mesh transition for a homogeneous material. (a) Bad cloud due to double transition (asymmetrical point distribution). (b) Three typical clouds shown for good transition. Red/Blue/Green nodes and associated clouds. Transition nodes shown in black.

\subsubsection{Atar build of large optical model}

An example of building a combined Atar-FCM model is shown in Figs. 26 and 27. This is a large optical model including both rectilinear elements and circular elements. The device consists of optical waveguides fabricated in Silica on Silicon technology with an integrated heater creating a semi-circular tuneable Mach-Zehnder interferometer. The device also incorporates a semi-circular backside thermal via. Further details on the model can be found in Chapter 5.6.2.

In order to save computational resources, bilateral symmetry is used thus requiring only half of the model. The build is initially defined using Atar to create the basic 


\begin{tabular}{|c|c|c|c|c|c|c|c|c|c|}
\hline \multirow{2}{*}{0} & \multirow{2}{*}{0} & 0 & or & 0 & \multicolumn{5}{|c|}{ dolololoাচাচপ } \\
\hline & & 0 & or & 0 & 08 & go & किо & $\frac{10}{0}$ & ifo \\
\hline 0 & 0 & 0 & 0 & & & & $\frac{19}{010}$ & 00 & 910 \\
\hline 0 & 0 & 10 & $6-$ & & & 이으 & 1010 & $\begin{array}{l}01 \\
010\end{array}$ & $\frac{90}{90}$ \\
\hline 0 & 0 & 10 & or & 0 & $d$ & 0 & 8 & 09 & 8 \\
\hline 0 & 0 & 0 & 웅 & 0 & d & 0 & 0 & 0 & \\
\hline 0 & 0 & o & 0 & 0 & 0 & 0 & 0 & 0 & 0 \\
\hline 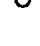 & 0 & 0 & 0 & 0 & 0 & 0 & 0 & 0 & o \\
\hline 0 & 0 & 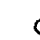 & & & & & 0 & 0 & \\
\hline
\end{tabular}

Figure 25: Atar mesh material transition. Red nodes are additional nodes added at the interface. The two clouds associated with one of the interface nodes are shown.

geometry. The Atar model defines the rectangular substrate consisting of a silicon substrate, a gold back contact and a top oxide layer. Mask definitions define the quadtree mesh used to refine the areas into which the semi-circular waveguide, heater and a backside thermal via are to be placed (Fig. 26(a)). This Atar model is then modified by the addition of curvilinear FCM elements creating a set of $3 \mathrm{D}$ volumes to define a thermal via with a gold centre, silicon sides and gold contacts (Fig. 26(b)). During the creation of the model any points within the volume of the defined arcs are cleared from the Atar model and the arcs are added creating a smooth curvilinear section on top of the rectilinear substrate. Finally, an FCM 3D arc is used to define a heater element on the top of the oxide (Fig. 27(d)). The entire model is shown in Fig. 27, and has on the order of one million points. 

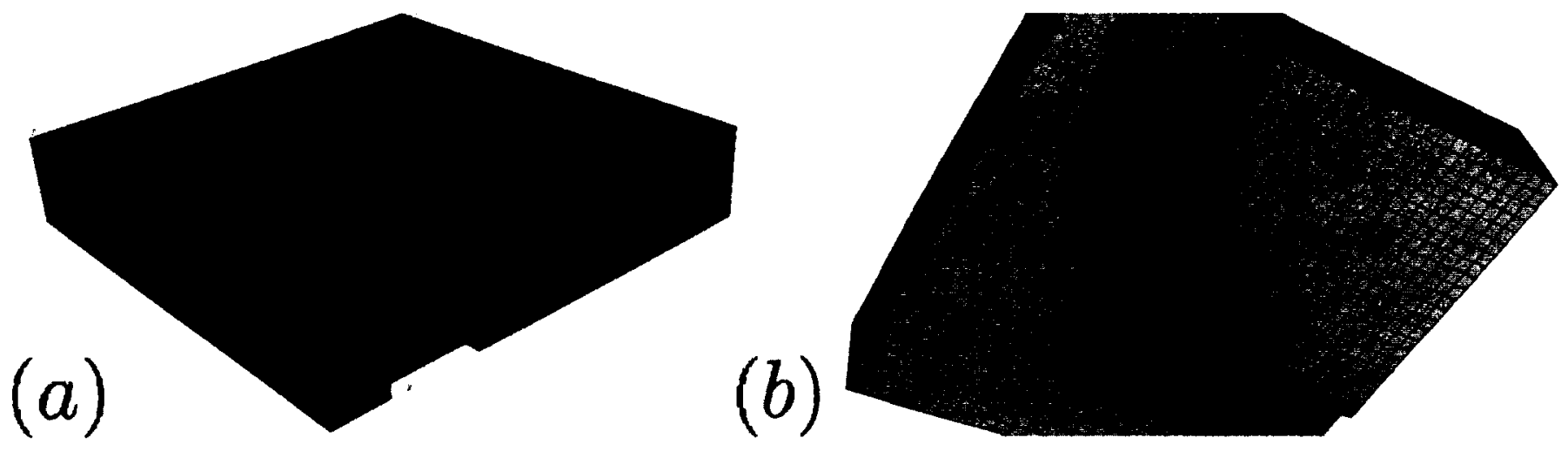

$(c)$
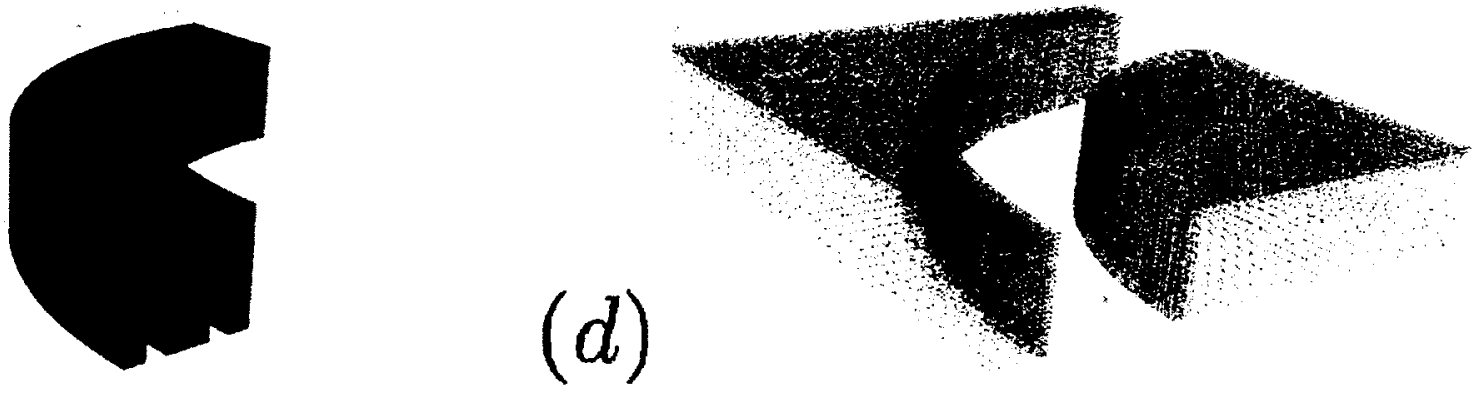

Figure 26: FCM build of semi-circular waveguide with heater and thermal via. Atar build of substrate showing quad-tree mesh. (a) Top view. (b) Bottom view. (c) FCM arc geometry consisting of gold and silicon arcs. (d) FCM point distribution derived from Atar quad-tree mesh with nodes removed for addition of arc nodes. 

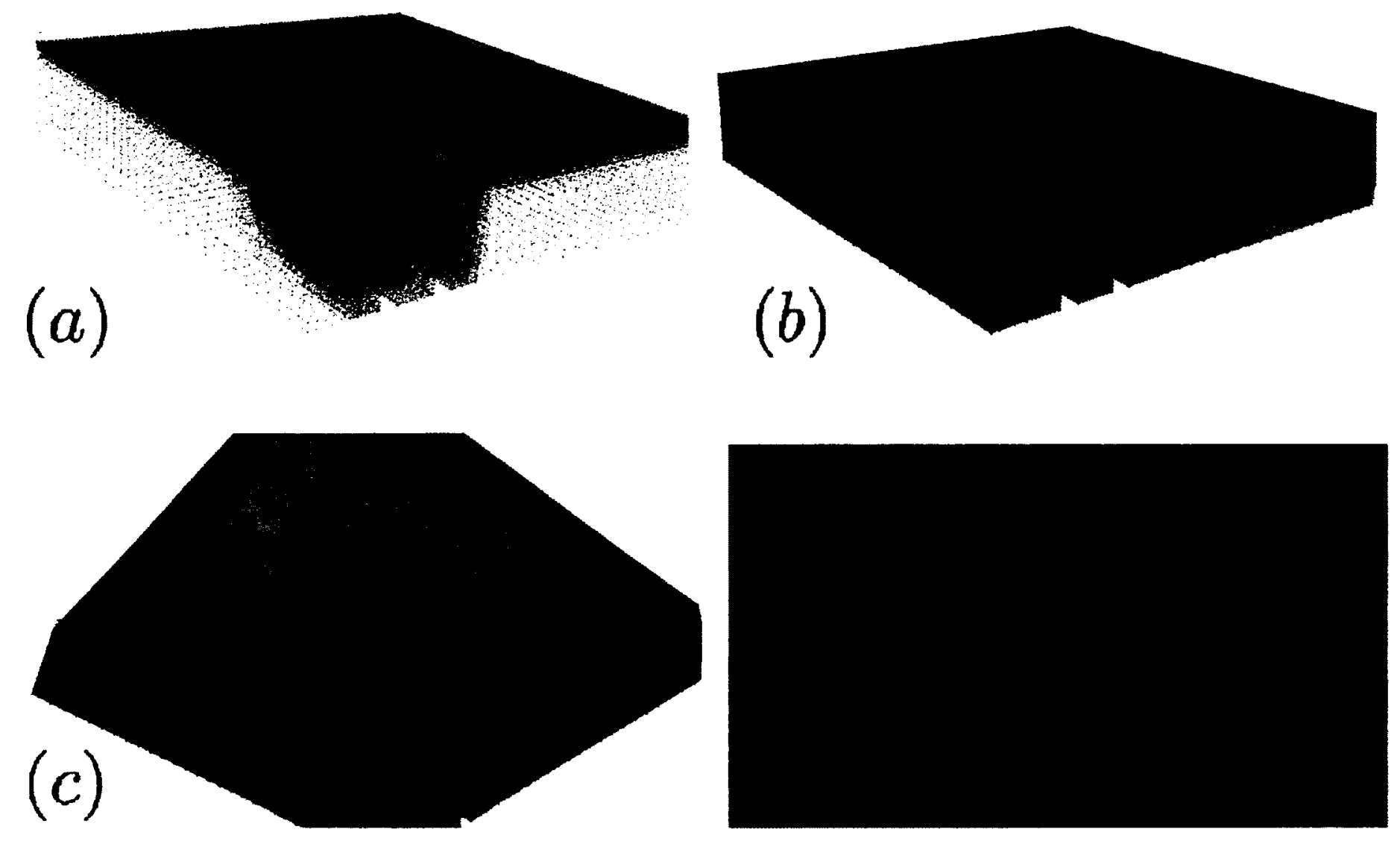

Figure 27: FCM point distribution for semi-circular waveguide. (a) All points. Surface and points. (b) Top view. (c) Bottom view. (d) Detail of point distribution for heater. 


\subsection{Initial tests}

This section presents the results for several cases of the Poisson equation in one- and two-dimensions. These results are used to ensure that the method behaves as expected, giving correct results and also to test the convergence of the method. These tests are also shown in the originating FCM paper [14], however I have redone them to test both the implementation of the FCM as well as the improvements and modifications to the method.

To measure the convergence we use a global error measure, previously described but for convenience shown here [14],

$$
\epsilon=\frac{1}{\left|u^{(e)}\right|_{\max } \sqrt{\frac{1}{N P} \sum_{I=1}^{N P}\left[u_{I}^{(e)}-u_{I}^{(c)}\right]^{2}}}
$$

with $\epsilon$ as the error in the solution, and the exact and computed solution denoted by superscript (e) and (c) respectively.

\subsubsection{1-D Poisson equation}

The first test case is a one-dimensional Poisson equation in $x$ with governing equation and boundary conditions

$$
\begin{array}{r}
\frac{\partial^{2} u}{\partial x^{2}}=\frac{105}{2} x^{2}-\frac{15}{2},-1<x<1 \\
u(-1)=1 \\
\frac{\partial u}{\partial x} \|_{x=1}=10
\end{array}
$$


and having an exact solution

$$
u=\frac{35}{8} x^{4}-\frac{15}{4} x^{2}+\frac{3}{8}
$$

The solution is approximated using a uniform series of points along the $x$ axis throughout the entire domain $-1 \leq x \leq 1$. The exact solution is plotted alongside the computed solution for $N P=202$ points, Fig. 28. Convergence of the solution and of the first derivative of the solution is shown in Fig. 29. The rate of convergence for $u$ is found to be 2.04 and the rate for $u_{x}$, the first derivative of the solution, is also found to be 2.04. The rate of conversion describes how the accuracy of a solution changes with increasing number of data points. A high rate of convergence is ideal as fewer points need to be added to achieve an improvement in solution accuracy. This rate of convergence is in agreement with those found in [14] which were all roughly equal to 2 .

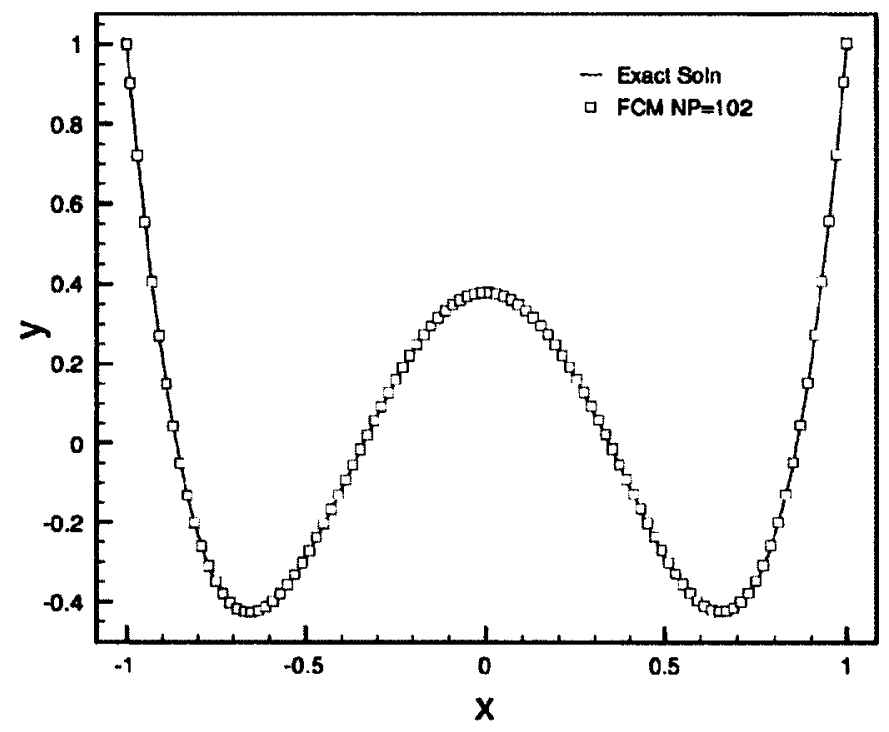

Figure 28: Exact and computed solution for the one dimensional Poisson equation.

A second test of the one-dimensional Poisson equation uses an example with a local 


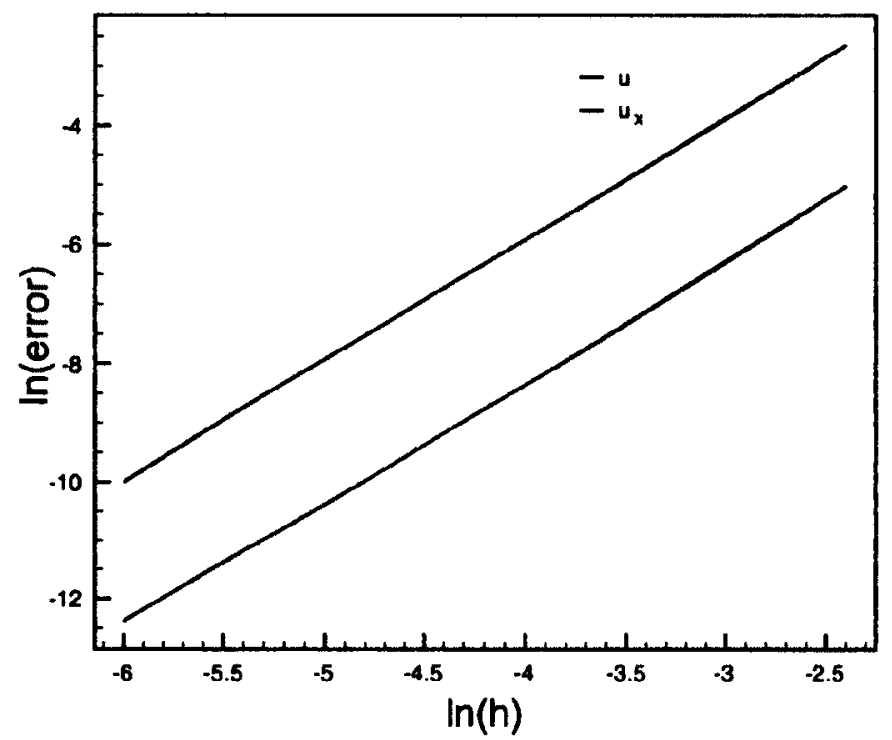

Figure 29: Convergence of the FCM for the one dimensional Poisson equation, with $h$ the internode spacing.

area of high solution gradient. The governing equation and boundary conditions are

$$
\begin{array}{r}
\frac{\partial^{2} u}{\partial x^{2}}=-6 x-\left[\frac{2}{\alpha^{2}}-4\left(\frac{x-\beta}{\alpha^{2}}\right)^{2}\right] \exp \left[-\left(\frac{x-\beta}{\alpha}\right)^{2}\right], 0 \leq x \leq 1 \\
u(0)=\exp \left(-\frac{\beta^{2}}{\alpha^{2}}\right) \\
\frac{\partial u}{\partial x} \|_{x=1}=-3-2\left(\frac{1-\beta}{\alpha^{2}}\right) \exp \left[-\left(\frac{1-\beta}{\alpha}\right)^{2}\right]
\end{array}
$$

and having an exact solution

$$
u=-x^{3}+\exp \left[-\left(\frac{x-\beta}{\alpha}\right)^{2}\right]
$$

For the following results we have used $\beta=0.5$ and $\alpha=0.05$. The computed solution using $N P=202$ points compared with the exact solution is shown in Fig. 30 and the convergence in Fig. 31. The convergence rate for the solution $u$ is found to be 2.15 
and for the first derivative 2.14 .

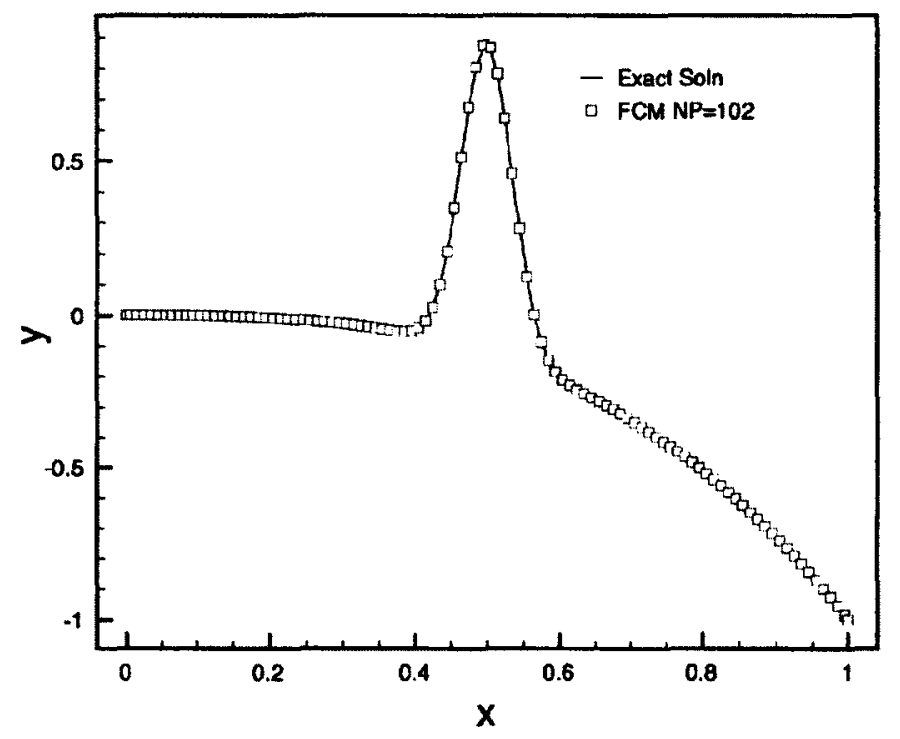

Figure 30: Exact and computed solution for the one dimensional Poisson equation with a region of high solution gradient.

\subsubsection{2-D Poisson equation}

Similar to the 1-D solutions, two tests of $2-\mathrm{D}$ equations are presented below. The first is a two dimensional Laplace equation

$$
\frac{\partial^{2} u}{\partial x^{2}}+\frac{\partial^{2} u}{\partial y^{2}}=0,0 \leq x \leq 1
$$

with Dirichlet boundary conditions

$$
\begin{array}{r}
u(0, y)=-y^{3} \\
u(1, y)=-1-y^{3}+3 y^{2}+3 y \\
u(x, 0)=-x^{3} \\
u(x, 1)=-1-x^{3}+3 x^{2}+3 x
\end{array}
$$




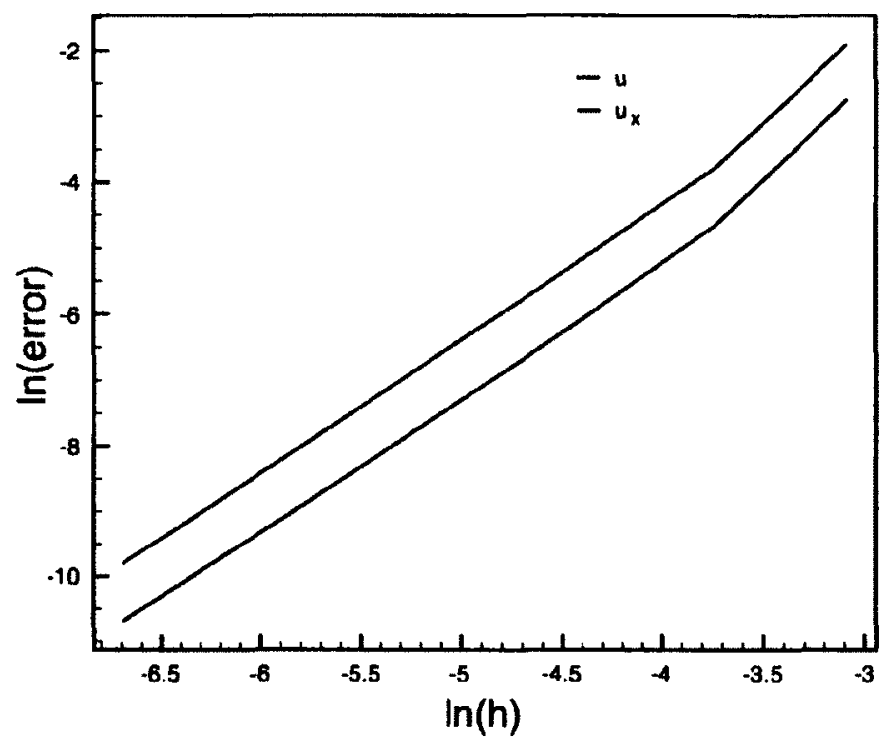

Figure 31: Convergence of the FCM for the one dimensional Poisson equation with a region of high solution gradient, with $h$ the internode spacing.

and the exact solution given by

$$
u(x, y)=-x^{3}-y^{3}+3 x y^{2}+3 x^{2} y
$$

A comparison between the exact and computed solutions is shown in Fig. 32 . The convergence of the solution is shown in Fig. 33 and the convergence rate for the solution is found to be 2.95 with the convergence of the $\mathrm{x}$ and $\mathrm{y}$ derivatives found to be 2.68 .

Finally, we test a two dimensional Poisson equation with a local area of high solution gradient, including both Neumann and Dirichlet boundary conditions. The 


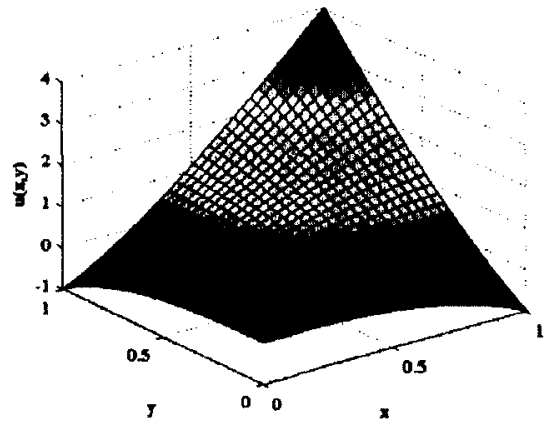

(a)

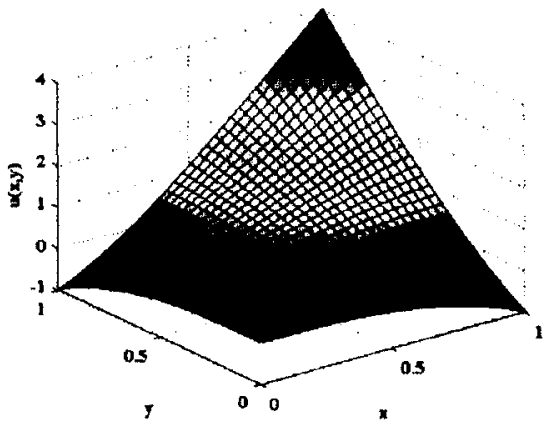

(b)

Figure 32: Exact, (a), and computed, (b), solution for the two dimensional Laplace equation.

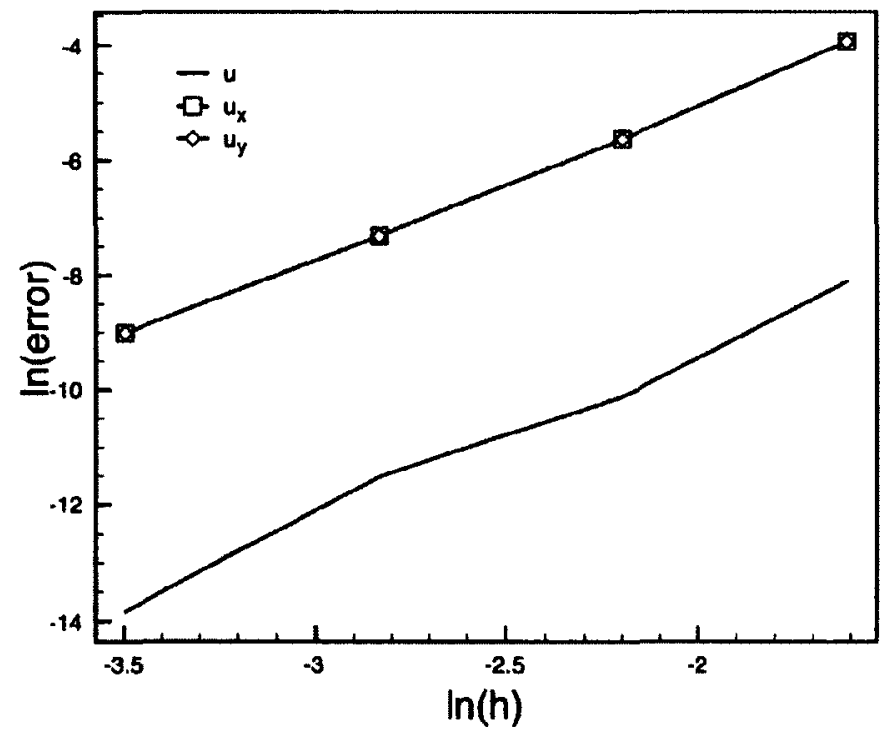

Figure 33: Convergence of the FCM for the two dimensional Laplace equation with $h$ the internode spacing. 
governing equations are

$$
\begin{array}{r}
\frac{\partial^{2} u}{\partial x^{2}}=-6 x-6 y-\left[\frac{4}{\alpha^{2}}-4\left(\frac{x-\beta}{\alpha^{2}}\right)^{2}-4\left(\frac{y-\beta}{\alpha^{2}}\right)^{2}\right] \\
\times \exp \left[-\left(\frac{x-\beta}{\alpha}\right)^{2}-\left(\frac{y-\beta}{\alpha}\right)^{2}\right], 0 \leq x \leq 1 \\
u(0, y)=-y^{3}+\exp \left[-\left(\frac{\beta}{\alpha}\right)^{2}-\left(\frac{y-\beta}{\alpha}\right)^{2}\right] \\
u(1, y)=-1-y^{3}+\exp \left[-\left(\frac{1-\beta}{\alpha}\right)^{2}-\left(\frac{y-\beta}{\alpha}\right)^{2}\right] \\
u_{y}(x, 0)=\frac{2 \beta}{\alpha^{2}} \exp \left[-\left(\frac{\beta}{\alpha}\right)^{2}-\left(\frac{x-\beta}{\alpha}\right)^{2}\right] \\
u_{y}(x, 1)=-3-2 \frac{1-\beta}{\alpha^{2}} \exp \left[-\left(\frac{x-\beta}{\alpha}\right)^{2}-\left(\frac{1-\beta}{\alpha}\right)^{2}\right]
\end{array}
$$

and the exact solution for this problem being

$$
u(x, y)=-x^{3}-y^{3}+\exp \left[-\left(\frac{x-\beta}{\alpha}\right)^{2}-\left(\frac{y-\beta}{\alpha}\right)^{2}\right]
$$

A comparison is shown between the exact solution, Fig. 34(a), and the computed solution, Fig. 34(b). The convergence of the solution and its first derivatives using a regular distribution of points is shown in Fig. 35. The convergence rate of the solution is found to be 2.07 and the rate for the derivatives of the solution is 2.25 . 


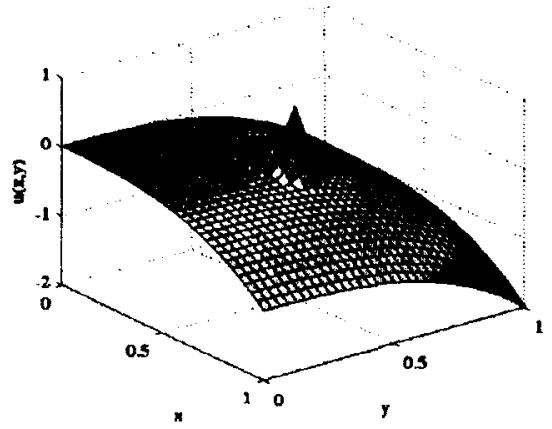

(a)

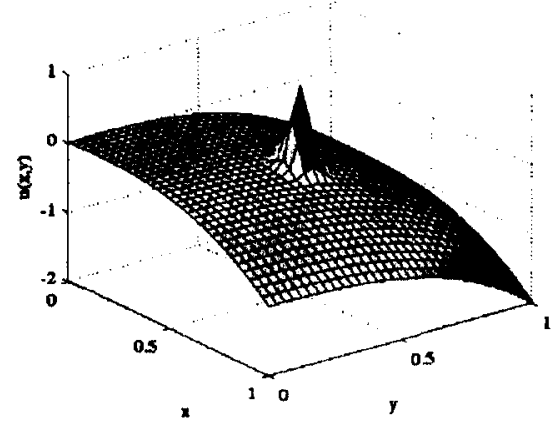

(b)

Figure 34: Exact, (a), and computed, (b), solution for the two dimensional Poisson equation with a region of high solution gradient.

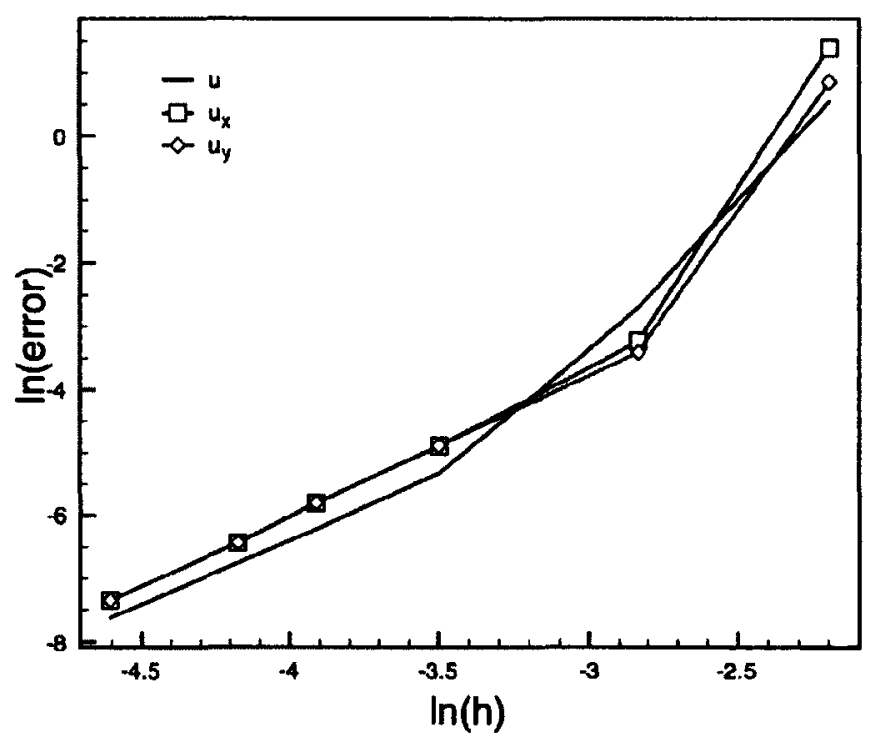

Figure 35: Convergence of the FCM for the two dimensional Poisson equation with a region of high solution gradient with $h$ the internode spacing. 


\section{Chapter 5}

\section{Thermal diffusion models}

This chapter explores the use of the finite cloud method on the thermal diffusion equation for materially inhomogeneous models. Previously published uses for the FCM have focused on solving materially homogeneous problems, in particular for the Poisson equation $[14,15,29]$. As thus far seen, apart from a one dimensional quasi-inhomogeneous BEM/FCM solution [26], discussed in section 3.2, the method has not been adapted for materially inhomogeneous problems, which is the basis of this chapter. We will first begin by discussing the method used to treat materially inhomogeneous problems, and then will apply this method to heat transfer problems in stationary and transient states.

During these tests a discrepancy was noted for certain models, in particular mesas, which resulted in erroneous thermal profiles. A thorough exploration of this problem is presented along with explanations and a correction factor. Finally, more complicated examples using large optical models are presented. These large models make very good use of the FCM as each piece of the model can be mapped out individually, such as the curvilinear sections and simply merged with the rectilinear pieces. As will be seen, no additional mapping or remeshing is required. As well, an advanced technique for model order reduction (MR) is demonstrated which is very helpful for 
solving the large models.

\subsection{Materially inhomogeneous FCM}

Many PDEs are valid for areas with only a single material property, such as thermal conductivity, described as being materially homogeneous. It is necessary, however, to be able to solve models or domains containing differing materials, materially inhomogeneous problems. Although the governing equations are typically for materially homogeneous regions, there also exist known physical relations for these situations which relate field parameters across material interfaces.

A method I propose for solving these multi-material or inhomogeneous problems involves creating distinct homogeneous regions for each material and then enforcing the interface conditions on the points connecting the materials. There are then three different types of nodes: internal nodes, external boundary or edge nodes and finally interface nodes which are used to 'stitch' the differing regions together. I will refer to this method of solving inhomogeneous problems as the stitched method, or s-FCM. During cloud creation, the clouds are restricted to nodes within their own material region and can include boundary nodes and interface nodes. Interface nodes are considered to be included in all regions which they touch. An example showing two separate regions and their corresponding clouds as done with the stitched method is shown in Fig. 36.

With the clouds known, they can be used to enforce the interface conditions on their given line of the shape matrix, similar to how interior nodes are used with the homogeneous conditions.

A simple example of an inhomogeneous model using the FCM and stitched interface conditions will now be provided. The same equation as Chapter 3.3, Eq. (31), 


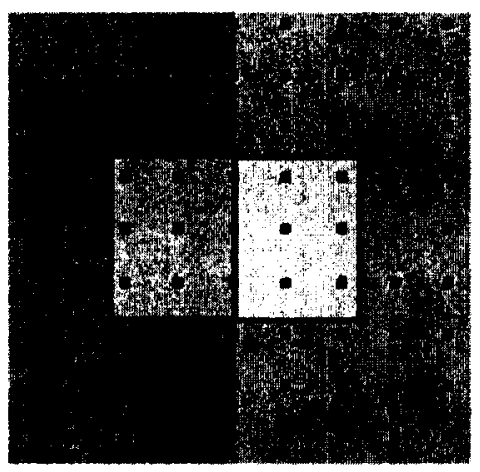

Figure 36: Nodes separated into two materially different regions, having interface nodes and two clouds, each extending into only one region.

is used however with a conductivity included as in

$$
\kappa \frac{\partial^{2} u(x, t)}{\partial x^{2}}=-B
$$

with $\kappa$ the conductivity and the matrix version being

$$
\kappa \mathbf{N}_{\mathbf{x x}} \mathbf{U}=-\mathbf{B}
$$

We will now include 8 points identified as $n_{1}$ to $n_{8}$ and Dirichlet boundary conditions using the forcing function $\mathbf{B}(1)=1.0$ and $\mathbf{B}(8)=0$. Points $n_{1}$ through $n_{4}$ are of material $a$ with $\kappa_{a}=1 \mathrm{~W} / \mathrm{m}-\mathrm{K}$, points $n_{5}$ through $n_{8}$ are material $b$ with $\kappa_{b}=2 \mathrm{~W} / \mathrm{m}$ K. An extra point in between nodes $n_{4}$ and $n_{5}$ will be added for the interface junction, $n_{j}$ at $x_{j}=4.5$, and is included in both materials.

For the interior nodes, $n_{2}, n_{3}, n_{6}$ and $n_{7}$, the shape function is calculated the same 
as in 3.3 giving

$$
\begin{aligned}
& N_{I, x x}(1)=\left[\begin{array}{lll}
0 & 0 & 2
\end{array}\right] \mathbf{M}^{-1}\left[\begin{array}{lll}
1 & 1 & 1
\end{array}\right]^{T} \varphi(-1)=1.0 \\
& N_{I, x x}(2)=\left[\begin{array}{lll}
0 & 0 & 2
\end{array}\right] \mathbf{M}^{-1}\left[\begin{array}{lll}
1 & 2 & 4
\end{array}\right]^{T} \varphi(0)=-2.0 \\
& N_{I, x x}(3)=\left[\begin{array}{lll}
0 & 0 & 2
\end{array}\right] \mathbf{M}^{-1}\left[\begin{array}{lll}
1 & 3 & 9
\end{array}\right]^{T} \varphi(1)=1.0
\end{aligned}
$$

which is the shape function for node $n_{2}$ in particular. The boundary nodes have Dirichlet conditions on them resulting in $N_{I}\left(x_{I}\right)=1$ with the remaining values of $N_{I}\left(x_{a}\right)=0, x_{a} \neq x_{I}$. The only nodes requiring new calculations are $n_{4}, n_{6}$ and $n_{j}$, the last of which will require two calculations as it is part of two materials and thus requires two clouds. The calculations for $n_{4}$ and $n_{6}$ will not be given as the process is the same as previously shown with a slightly different nodal spacing. The first calculation for $n_{j}$ begins with the $\mathbf{F}$ matrix using nodes $n_{3}, n_{4}$ and $n_{j}$

$$
\mathbf{F}=\left[\begin{array}{ccc}
1 & 1 & 1 \\
3 & 4 & 4.5 \\
9 & 16 & 20.25
\end{array}\right]
$$

The $\mathrm{W}$ matrix is found using a Kernel centred at $x_{K}=4.5$ giving

$$
\mathbf{W}=\left[\begin{array}{ccc}
3.859 \mathrm{E}-7 & 0 & 0 \\
0 & 0.2383 & 0 \\
0 & 0 & 1.2615
\end{array}\right]
$$


The moment matrix is then calculated as $\mathbf{M}=\mathbf{F W F}^{\mathbf{T}}$ resulting in

$$
\mathbf{M}=10^{2} \times\left[\begin{array}{ccc}
0.015 & 0.066 & 0.294 \\
0.066 & 0.294 & 1.302 \\
0.294 & 1.302 & 5.783
\end{array}\right]
$$

For interface nodes we are interested in the physical constraints for the junction, in this case heat flow must be conserved across the materials giving

$$
\begin{aligned}
\left.\kappa_{a} \nabla u\right|_{a} & =\left.\kappa_{b} \nabla u\right|_{b} \\
\left.\kappa_{a} \nabla u\right|_{a}-\left.\kappa_{b} \nabla u\right|_{b} & =0,
\end{aligned}
$$

for a material $a$ and material $b$. The second line of Eq. (73) is what will be enforced in the system of equations, and will require first order derivatives. We find the shape function for node $n_{j}$ to be

$$
\begin{aligned}
N_{a, I, x}(3) & =[019] \mathbf{M}^{-1}[139]^{T} \varphi(-1.5)=0.333 \\
N_{a, I, x}(4) & =[019] \mathbf{M}^{-1}[1416]^{T} \varphi(-0.5)=-3.0 \\
N_{a, I, x}(4.5) & =[019] \mathbf{M}^{-1}[14.520 .25]^{T} \varphi(0)=2.666
\end{aligned}
$$

The calculations for the shape function for nodes $n_{j}, n_{5}, n_{6}$ will not be shown however the results are

$$
\begin{aligned}
N_{b, I, x}(4.5) & =\left[\begin{array}{lll}
0 & 1 & 9
\end{array}\right] \mathbf{M}^{-1}[14.520 .25]^{T} \varphi(0)=-2.666 \\
N_{b, I, x}(5) & =\left[\begin{array}{lll}
0 & 1 & 9
\end{array} \mathbf{M}^{-1}[1525]^{T} \varphi(0.5)=3.0\right. \\
N_{b, I, x}(6) & =\left[\begin{array}{lll}
0 & 1 & 9
\end{array} \mathbf{M}^{-1}[1636]^{T} \varphi(1.5)=-0.333\right.
\end{aligned}
$$


The actual matrix equation for the line corresponding to node $n_{j}$ is

$$
\left[\kappa_{a} \mathbf{N}_{\mathbf{a}, \mathbf{x}}-\kappa_{b} \mathbf{N}_{\mathbf{b}, \mathbf{x}}\right] \mathbf{U}=0
$$

resulting in the shape matrix

$$
\begin{aligned}
& \mathbf{N}=\left[\begin{array}{llll}
\mathbf{I} & & \\
& & & \\
& \kappa_{a} \mathbf{N}_{\mathbf{x x}} & \\
\kappa_{a} \mathbf{N}_{\mathbf{a}, \mathbf{x}} & -\kappa_{b} \mathbf{N}_{\mathbf{b}, \mathbf{x}} & \\
& & \kappa_{b} \mathbf{N}_{\mathbf{x x}} & \\
& & & \mathbf{I}
\end{array}\right]
\end{aligned}
$$

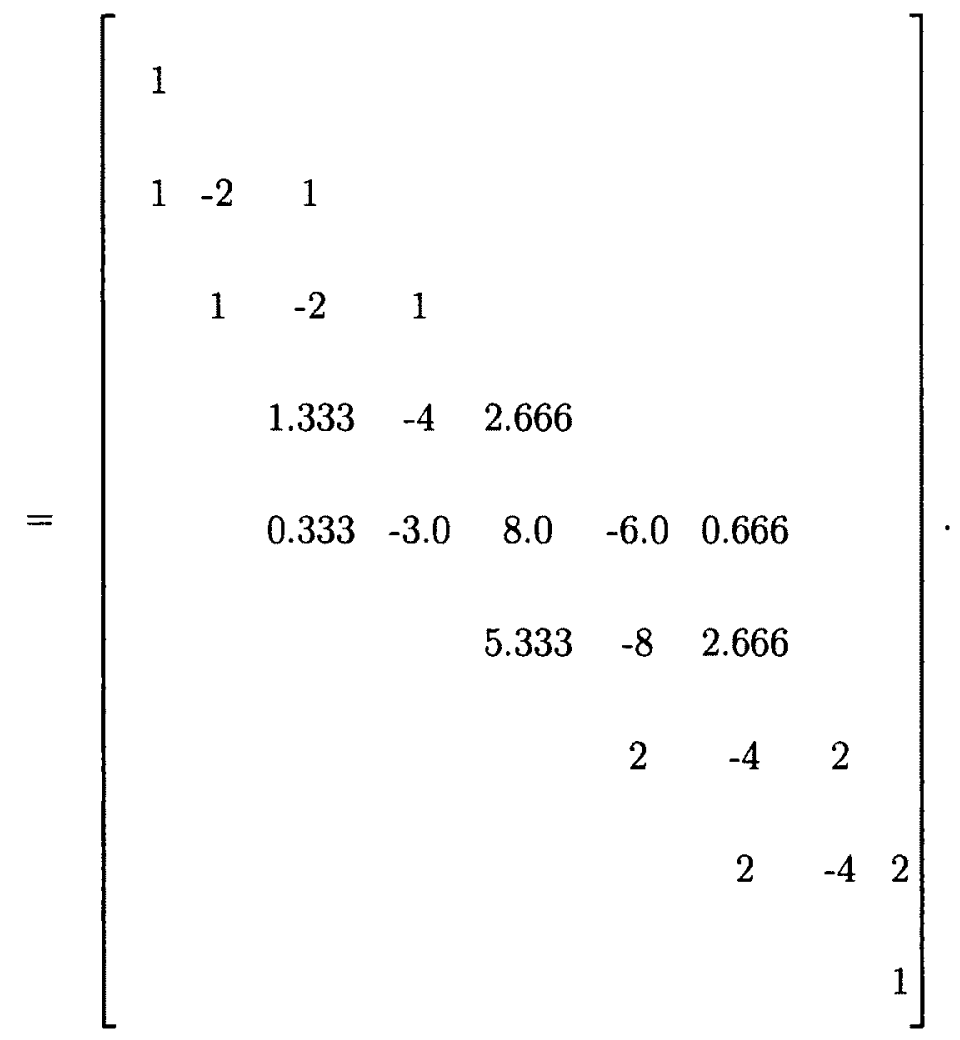


Finally, calculating the solution as before,

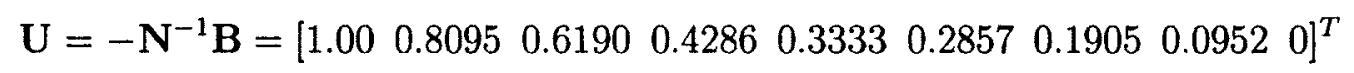

giving a linear slope in the interior regions and twice the slope in region $a$ than in region $b$, exactly as expected.

\subsection{Heat diffusion equation}

The simplest form of the heat flow equation describes the diffusion of heat in a region of constant thermal conductivity $(\kappa)$ and capacitance $(C)$ and is given by,

$$
C \frac{\partial u}{\partial t}=\kappa \nabla^{2} u+\rho(\mathbf{r}, \mathbf{t})
$$

with a heat source or forcing function described by $\rho$, previously $B$.

Within each region of constant material parameters Eq. (80) will hold, and a three dimensional discretized version of the equation for the temperatures on internal nodes $\left(T_{I}\right)$ is given by,

$$
C \frac{\partial \mathbf{T}_{\mathbf{I}}}{\partial t}=\kappa\left[\mathbf{N}_{\mathbf{x x}}+\mathbf{N}_{\mathbf{y y}}+\mathbf{N}_{\mathbf{z z}}\right] \mathbf{T}_{\mathbf{I}}+\mathbf{R}(\mathbf{t})
$$

where $\mathbf{N}_{\mathbf{x x}}, \mathbf{N}_{\mathbf{y y}}$ and $\mathbf{N}_{\mathbf{z z}}$ are the shape matrix operators for the second spatial derivatives and $\mathbf{R}$ the heat generation at every node in the region.

At the boundary nodes of each region three types of conditions can be applied. The simplest boundary condition is the previously mentioned Dirichlet condition where,

$$
\mathbf{T}_{\mathbf{D}}=\mathbf{T}_{\mathbf{0}}
$$


and $\mathbf{T}_{\mathbf{D}}$ are the temperatures of the boundary nodes subject to a Dirichlet condition and $\mathbf{T}_{\mathbf{0}}$ a vector of the boundary temperatures.

A subject that has yet to be described is the method for dealing with Neumann boundary conditions or stitched interface conditions in more than one dimension. For this added layer of complexity the boundary or interface normals must be known and applied appropriately to the derivative operator matrices. The condition for a Neumann boundary condition which establishes a fixed heat flow $\left(f_{B}\right)$ across a boundary is

$$
\kappa \nabla T \cdot \hat{\mathbf{n}}_{\mathbf{B}}=f_{B}
$$

which, using the FCM formulation, becomes for each boundary node

$$
\kappa\left[\left(\left.\mathbf{N}_{\mathbf{x}}\right|_{n} \hat{i}+\left.\mathbf{N}_{\mathbf{y}}\right|_{n} \hat{j}+\left.\mathbf{N}_{\mathbf{z}}\right|_{n} \hat{k}\right) \mathbf{T}\right] \cdot \hat{\mathbf{n}}_{\mathbf{B}}=\left.\mathbf{f}_{\mathbf{B}}\right|_{n}
$$

where, for example, $\left.\mathbf{N}_{\mathbf{x}}\right|_{n}$ is a vector associated with the n'th row of the first order shape matrix $\mathbf{N}_{\mathbf{x}}, \hat{\mathbf{n}}_{\mathbf{B}}$ the normal at the boundary and $\left.\mathbf{f}_{\mathbf{B}}\right|_{n}$ the flow normal to the surface for n'th node. Adiabatic no flow boundaries would set $f_{B}$ to zero.

The final boundary condition that can be present is at a material interface. Across these boundaries the heat flow normal to the boundaries must be equal:

$$
\left.\kappa_{a} \nabla T\right|_{a} \cdot \hat{\mathbf{n}}_{\mathbf{a}}-\left.\kappa_{b} \nabla T\right|_{b} \cdot \hat{\mathbf{n}}_{\mathbf{b}}=0
$$

where " $a$ " and " $b$ " denote the two material regions on either side of the interface.

To implement this condition at the interface between two regions, common nodes 
will be placed and for these nodes Eq. (85) becomes,

$$
\begin{aligned}
& \kappa_{a}\left[\left(\left.\mathbf{N}_{\mathbf{x}}^{\mathbf{a}}\right|_{n} \hat{i}+\left.\mathbf{N}_{\mathbf{y}}^{\mathbf{a}}\right|_{n} \hat{j}+\left.\mathbf{N}_{\mathbf{z}}^{\mathbf{a}}\right|_{n} \hat{k}\right) \mathbf{T}_{a}\right] \cdot \hat{\mathbf{n}}_{\mathbf{a}}- \\
& \kappa_{b}\left[\left(\left.\mathbf{N}_{\mathbf{x}}^{\mathbf{b}}\right|_{n} \hat{i}+\left.\mathbf{N}_{\mathbf{y}}^{\mathbf{b}}\right|_{n} \hat{j}+\left.\mathbf{N}_{\mathbf{z}}^{\mathbf{b}}\right|_{n} \hat{k}\right) \mathbf{T}_{b}\right] \cdot \hat{\mathbf{n}}_{\mathbf{b}}=0
\end{aligned}
$$

where each material ("a" or "b") has its associated shape matrices and temperature vector.

To form a heat flow model of a complex structure the Eqs. (81), (82), (84) and (86) can be incorporated into a global set of equations described by,

$$
\mathbf{C} \frac{\partial \mathbf{T}}{\partial t}=\mathbf{G T}+\mathbf{B}+\sum_{j=1}^{q} \mathbf{R}_{\mathbf{j}}(\mathbf{t})
$$

For a structure with multiple regions the $\mathbf{T}$ vector would have the form of $\mathbf{T}=$ $\left[\mathbf{T}_{\mathbf{a}} \mathbf{T}_{\mathbf{b}} \ldots\right]$ and we have $q$ independent heat sources each described by a vector $\mathbf{R}_{\mathbf{j}}$.

\subsection{Transient solutions}

With the time dependent equation, a time integration or time stepping method must be used to solve the model at subsequent steps, as the system evolves with time. A typical integration method used for transient analysis of the thermal diffusion equation is backward Euler [6]. Every integration technique has benefits and drawbacks to its use, such as the order of the error in each time step, or the size of its regions of stability. Regions of stability restrict the size of time step allowed, to prevent diverging or oscillating solutions.

An advantage of backward Euler is that its region of stability encompasses the entire left half plane, which allows for as large a time step as desired without adding any instabilities (accuracy, however, will be lowered with larger time steps). As will 
be shown the method does require a matrix decomposition for its solution and is thus dubbed an implicit method. The formulation is described as such [6]

$$
u^{n+1}=u^{n}+\Delta t u_{t}^{n+1}
$$

with $\Delta t$ the size of the time step between current time $n$ and the following step $n+1$. We rearrange to keep the time steps of $n$ on one side of the equation and $n+1$ on the other,

$$
\frac{1}{\Delta t} u^{n}=\frac{1}{\Delta t} u^{n+1}-u_{t}^{n+1}
$$

We substitute, as an example, a simple PDE of $u_{x x}=u_{t}$ into the above equation giving

$$
\frac{1}{\Delta t} u^{n}=\frac{1}{\Delta t} u^{n+1}-u_{x x}^{n+1}
$$

With more complicated PDEs the substition remains the same but may carry more terms or constants. Next, rearranging and putting the equations into a discrete form we have

$$
\frac{1}{\Delta t} \mathbf{U}^{n}=\frac{1}{\Delta t} \mathbf{U}^{n+1}-\mathbf{N}_{\mathbf{x x}} \mathbf{U}^{n+1}
$$

And again positioning the matrices into the final useful form for our purposes,

$$
\frac{1}{\Delta t} \mathbf{U}^{n}=\left[\frac{1}{\Delta t} \mathbf{I}-\mathbf{N}_{\mathbf{x x}}\right] \mathbf{U}^{n+1}
$$


and solution form,

$$
\frac{1}{\Delta t}\left[\frac{1}{\Delta t} \mathbf{I}-\mathbf{N}_{\mathbf{x x}}\right]^{-1} \mathbf{U}^{n}=\mathbf{U}^{n+1}
$$

This is implemented for Eq. (87) as

$$
\left[\frac{\mathbf{C}}{\Delta t}-\mathbf{G}\right]^{-1} \frac{\mathbf{C}}{\Delta t} \mathbf{T}^{\mathbf{n}}-\mathbf{B}-\sum_{j=1}^{q} \mathbf{R}_{\mathbf{j}}(\mathbf{t})=\mathbf{T}^{\mathbf{n}+\mathbf{1}}
$$

\subsection{Initial examples}

\subsubsection{Pseudo-1D steady state}

An initial test of the method is shown below and compared with theoretical results. A 3-D model was created of a rectangular bar with dimensions $(15 \mathrm{~m} \times 5 \mathrm{~m} \times 5 \mathrm{~m})$, conductivity $\kappa=1 \mathrm{~W} / \mathrm{m}-\mathrm{K}$ and a heat source in the middle giving $25 \mathrm{~W}$ evenly over the cross section. Each end of the bar was held at a constant temperature $(T=0 \mathrm{~K})$, with adiabatic Neumann boundary conditions on the side edges creating in essence a 1D heat flow. A diagram of the model can be seen in Fig. 37. The maximum rise in temperature is calculated as two thermal resistive elements of $(7.5 \mathrm{~m} \times 5 \mathrm{~m} \times 5 \mathrm{~m})$ in 'parallel'. The expected rise in temperature is calculated as

$$
\begin{aligned}
R_{t h} & =\frac{1}{\sigma} \frac{w}{l h}=\frac{1}{1}(\mathrm{~m}-\mathrm{K} / \mathrm{W}) \frac{7.5}{5 \times 5}\left(\mathrm{~m} / \mathrm{m}^{2}\right)=0.3 \mathrm{~K} / \mathrm{W} \\
T_{\text {rise }} & =R_{t h} \| R_{t h} \times P_{i n}=0.3 / 2(\mathrm{~K} / \mathrm{W}) \times 25(\mathrm{~W})=3.75 \mathrm{~K}
\end{aligned}
$$

giving the expected maximum temperature rise for this simulation to be $T_{\text {rise }}=$ $3.75 \mathrm{~K}$.

A heat profile the length of the block as calculated by the FCM is shown in Fig. 38. 


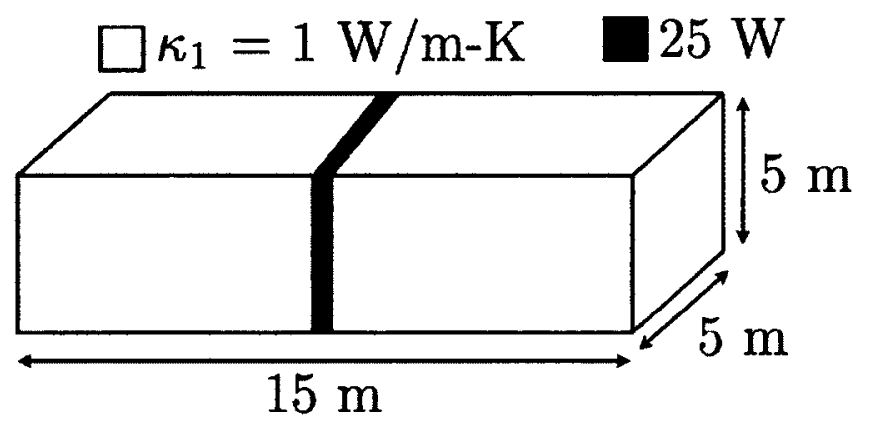

Figure 37: Diagram model for the simple homogeneous heat problem, with power added to darkened region in the middle.

The determined maximum temperature rise is shown to be equal to that calculated above.

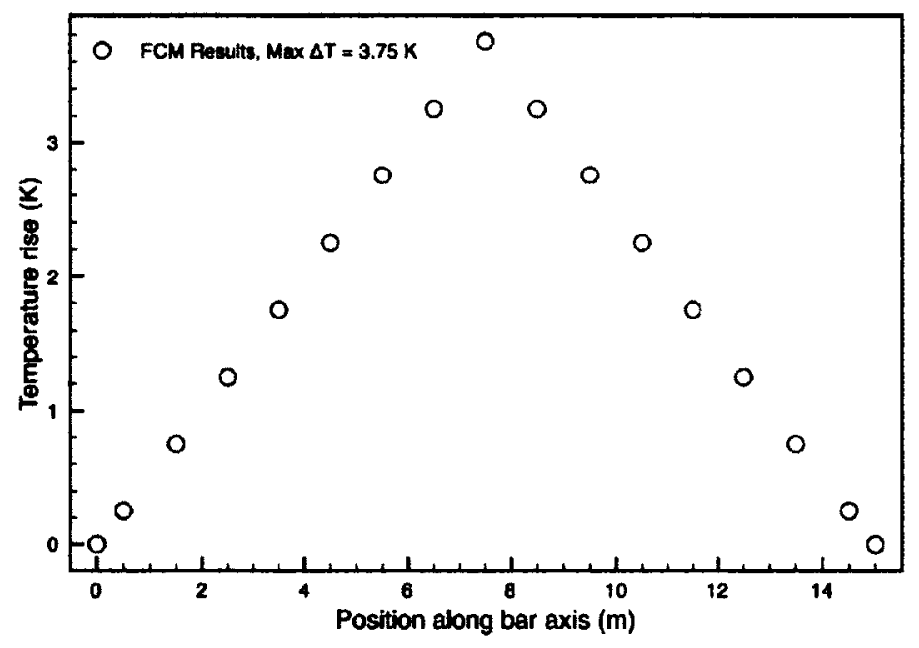

Figure 38: Temperature profile along the $\mathrm{x}$-axis for a homogeneous single block of dimensions $(15 \mathrm{~m} \times 5 \mathrm{~m} \times 5 \mathrm{~m})$ with $25 \mathrm{~W}$ of heat added to the mid section and a conductivity of $\kappa=1 \mathrm{~W} / \mathrm{m}-\mathrm{K}$.

A second test was performed to verify the functionality of the inhomogeneous model. The second model was created using two materials of $\kappa_{1}=1 \mathrm{~W} / \mathrm{m}-\mathrm{K}$ and $\kappa_{2}=2 \mathrm{~W} / \mathrm{m}-\mathrm{K}$ as shown in Fig. 39. Dimensions of the model are $(21 \mathrm{~m} \times 5 \mathrm{~m} \times 5 \mathrm{~m})$ with the seven metres in the middle being material 2, and the two outer sections being material 1 . The temperature rise is calculated similarly as above, and found to 
be $T_{\text {rise }}=4.375 \mathrm{~K}$, and this agrees exactly with the result found from the FCM. A temperature profile of the model running along the x-axis is shown in Fig. 40 .

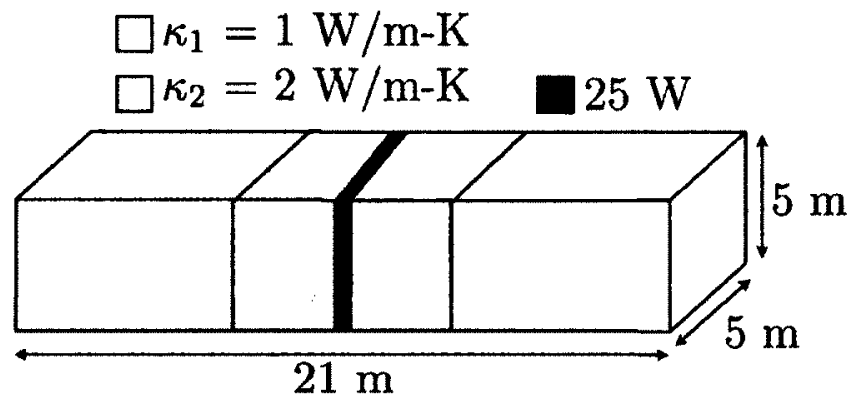

Figure 39: Diagram of the second heat problem for the inhomogeneous case of two materials. The two regions with different conductances are shown, with power added to the darkened region in the middle.

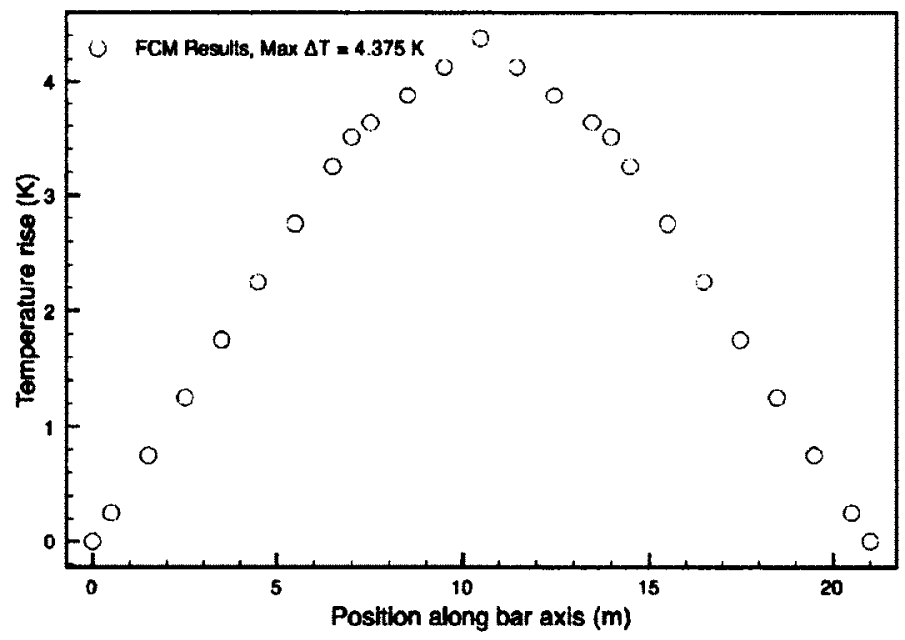

Figure 40: Temperature profile along the $\mathrm{x}$-axis for the inhomogeneous block of dimensions $(21 \mathrm{~m} \times 5 \mathrm{~m} \times 5 \mathrm{~m})$ with two different material conductivities and a heat source of $25 \mathrm{~W}$ in the middle.

\subsubsection{D steady state heat flow}

Although the above examples were modelled in full three dimensions, they are essentially $1 \mathrm{D}$ heat flow problems. A full $3 \mathrm{D}$ problem is next used to verify correct 
operation and results for situations with heat flowing at angles other than parallel and perpendicular to the node distribution and boundaries. It should be noted that the previous examples compared the computed solutions with analytic solutions giving an absolute error. The following examples are compared with other numerical techniques and are only able to show that the FCM solutions are in agreement with the other methods.

The following, Fig. 41(a)-(d), shows a model of size (25 $\mu \mathrm{m} \times 25 \mu \mathrm{m} \times 25 \mu \mathrm{m})$ with a ( $5 \mu \mathrm{m} \times 5 \mu \mathrm{m} \times 5 \mu \mathrm{m})$ source of $50 \mathrm{~mW}$. The bottom of the model, $z=0$, has a fixed Dirichlet condition of $T=0$ and adiabatic Neumann conditions on all other sides restricting heat flow exclusively to the bottom.

Results from this model are shown in a 1D plot with the rise in temperature along a line on the z-axis through the centre of the model. A comparison is made with Atar [8] using the same mapping for both models which allows for a more realistic comparison. Fig. 41(e) shows a very close agreement between the two tools.

\subsubsection{Transient heat flow}

A remaining initial test is needed to ensure that the transient solutions using backward Euler are accurate. A basic model, a rectangular prism, is created with five of the sides keeping adiabatic Neumann conditions. A final side at the maximum $x$ keeps those nodes at $T_{B}=0 \mathrm{~K}$ with Dirichlet conditions. Initial temperatures for the nodes are $T_{\text {inital }}=0 \mathrm{~K}$. Several of the internal nodes are identified and assigned a heat generation of $0.5 \mathrm{~W}$ at each node. The nodal layout is seen in Fig. 42(a), with pink nodes the adiabatic boundary points, red being the Dirichlet nodes, grey the internal nodes, and yellow nodes are identified as the heat generating points.

This model is created and solved using Atar, and the FCM, both using the same point spacings and the solutions running until reaching steady state. The temperature 
(a)

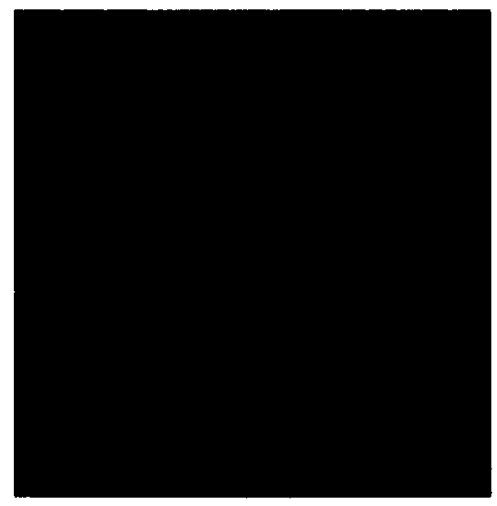

(b)

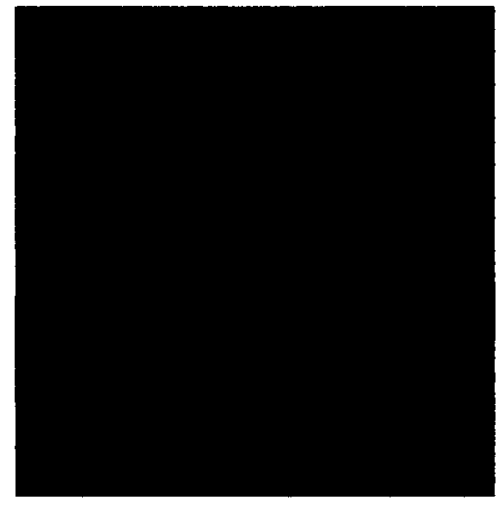

(c)
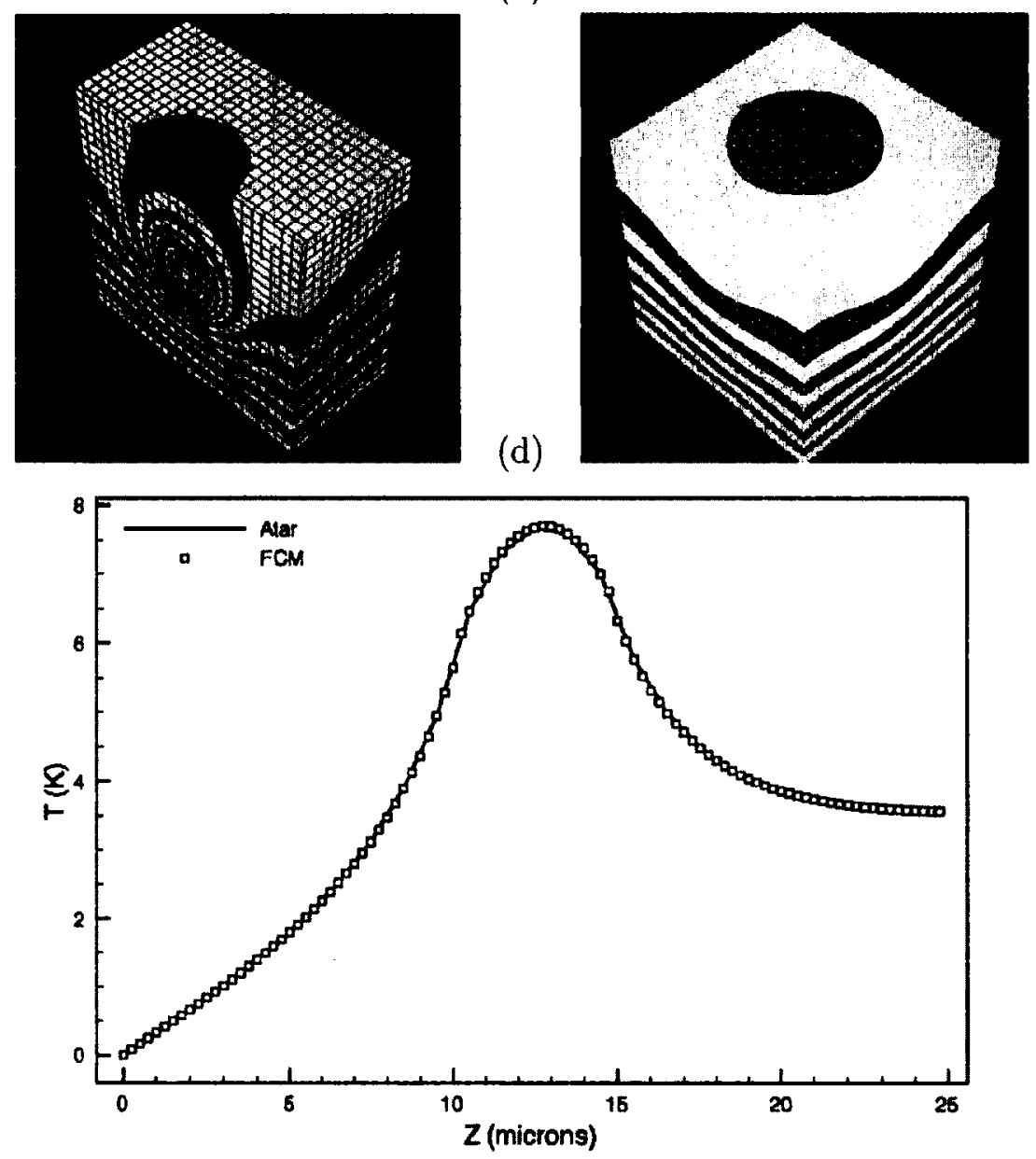

(e)

Figure 41: 3D heat flow in rectangular solid. (a)Atar model (Half view). (b) Meshless model. (c) Atar heat contours (Half view). (d) Meshless heat contours. (e) Temperature through block in $\mathrm{z}$ direction. 


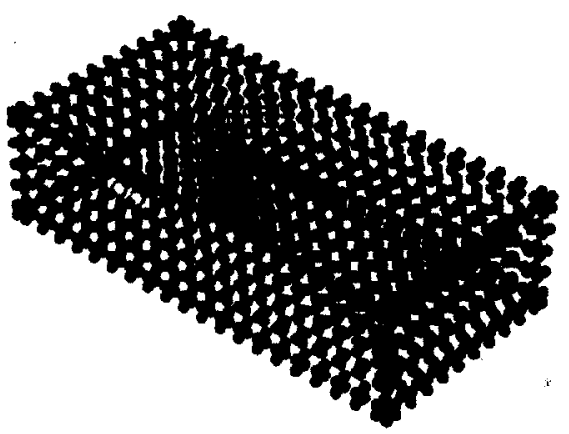

(a)

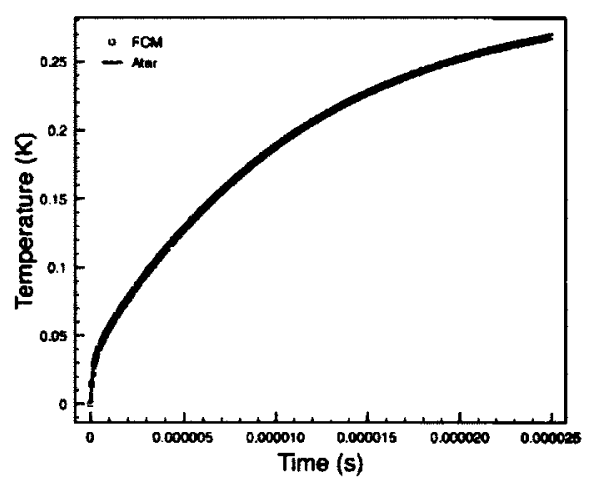

(b)

Figure 42: (a) Node placement for the simple transient analysis with pink nodes the adiabatic boundary points, red being the Dirichlet nodes, grey the internal nodes, and yellow nodes are identified as the heat generating points. (b) Transient response for both the Atar solution and the FCM solution.

of a node at $x=0$ and at the center of the face in the $z-y$ plane is compared between the two methods. A temperature vs. time plot is shown in Fig. 42(b), with the FCM agreeing nearly exactly with Atar.

\subsubsection{Gallium nitride power amplifier}

With the promising results already seen, a more thorough simulation was performed on a complex structure in three dimensions and directly compared with the results from Atar. The structure tested is an integrated gallium nitride power amplifier, shown in Fig. 43(a), with heat sources along six of the 'fingers'. Adiabatic Neumann boundary conditions are placed on all but one of the sides. Dirichlet boundary conditions are placed on the remaining side, the maximum $x$ side, forcing the temperature to $\mathrm{T}=0 \mathrm{~K}$.

A heat map with the FCM results is shown below in Fig. 43(b), the varying colours representing the rise in temperature across the top of the structure. A comparison of the two methods can be seen from a 1D cut along the $\mathrm{x}$-axis through the centre of the 

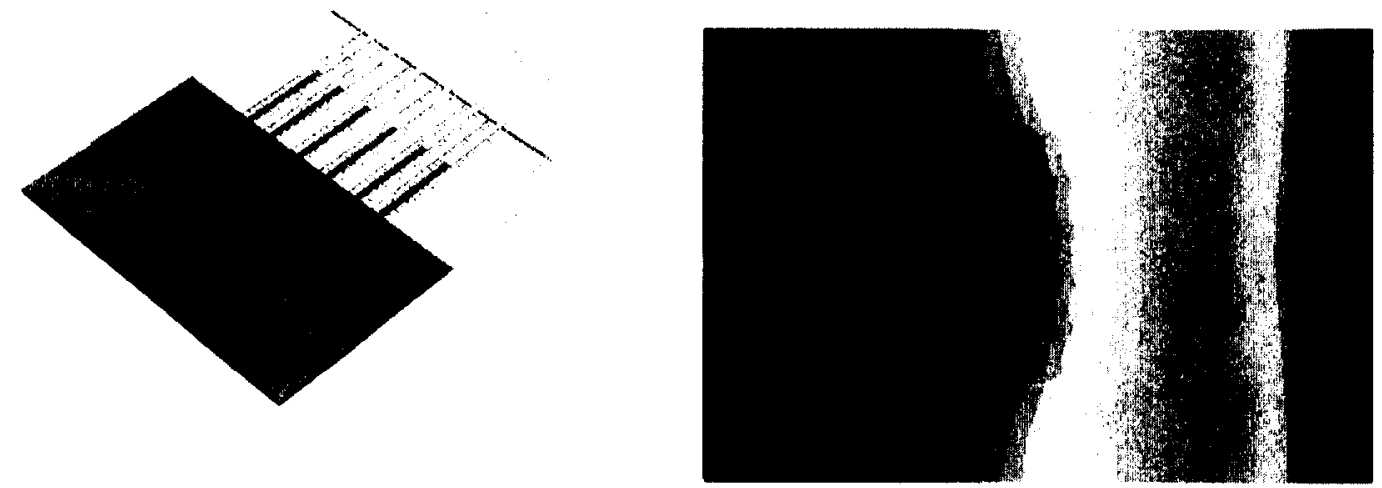

(a)

(b)

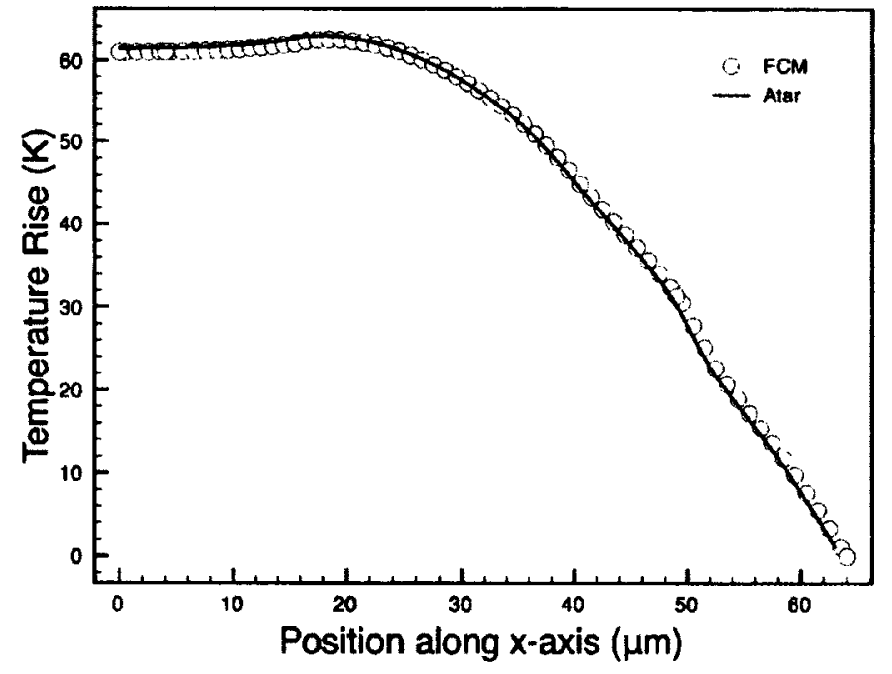

(c)

Figure 43: (a) Depiction of the 3D gallium nitride power amplifier used to compare the FCM results with Atar results. Red bars indicate locations of added power, with $2.4 \mathrm{~mW}$ added in each 'finger'. (b) Heat temperature plot showing FCM simulation results of amplifier. (c) Temperature profile comparison of the meshless and meshed methods for a $1 \mathrm{D}$ slice along the $\mathrm{x}$-axis through the centre of the amplifier. 


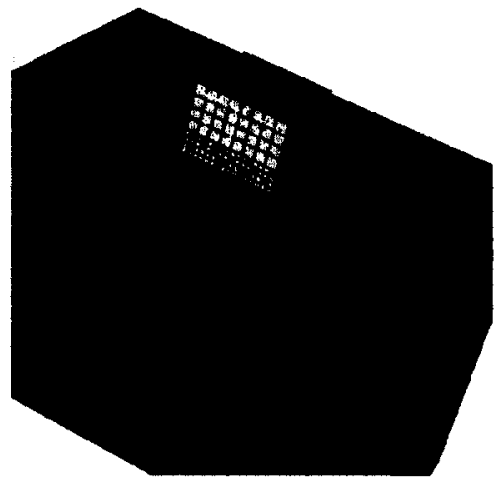

(a)

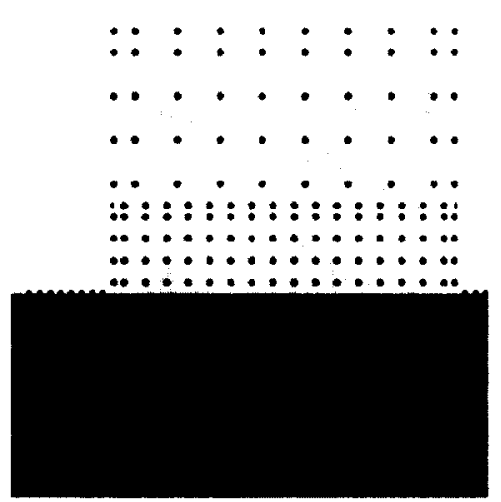

(b)

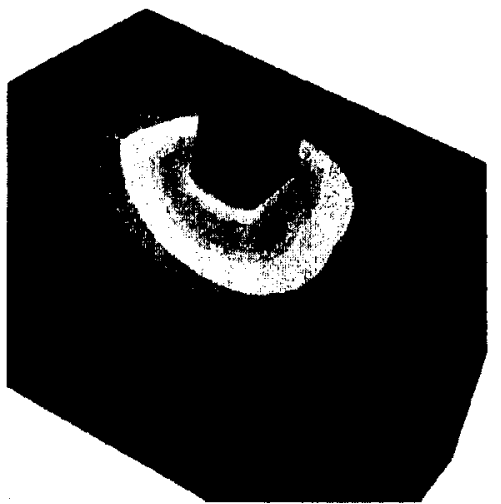

(c)

Figure 44: Mesa Structure. (a) Original course Atar mesh. (b) FCM point distribution. (c) Thermal distribution from FCM.

model as seen in Fig. 43(c). The temperature profile using the meshless method is in very close agreement with the Atar simulation using the same block/point layout, with the two results staying within $1 \%$ of each other.

\subsection{Mesa structures}

As mentioned in the introduction, certain errors were noticed when using a simple FCM formulation to solve a mesa type structure on top of a larger substrate. Depending on the situation, heat would be either generated or lost across the mesa junction. The following sections explore the situation, contributing factors to the error, and a solution mechanism.

\subsubsection{Basic mesa analysis}

The first stage in evaluating the use of the FCM for heat flow through mesa structures was to build a simple mesa structure as shown in Fig. 44. The thermal conductivities were $\kappa_{\text {mesa }}=100 \mathrm{~W} / \mathrm{m}-\mathrm{K}$ and $\kappa_{\text {sub }}=1 \mathrm{~W} / \mathrm{m}-\mathrm{K}$. The first figure shows the initial 
Atar mesh. For this model a heat source was placed at the very top of the mesa and the bottom surface was at a fixed temperature of zero. All other boundaries were adiabatic. A primary consideration of the heat flow in the structure is the heat spreading that occurs at the interface of the mesa and the substrate. Obtaining accurate temperature distributions requires refinement of the mesh or point distribution at this interface. Using the Atar block structure an FCM point distribution was created with a point at the centre of each block. Additional points were added on the model faces and at the transitions from larger blocks to smaller blocks. An Atar-FCM point distribution is shown in Fig. 44(b) with a contour plot presented in Fig. 44(c).

A comparison between Atar and Atar-FCM results for this structure (labeled Atar-2/FCM-2) is presented for a temperature profile along a line from the bottom of the structure through the centre of the mesa in Fig. 45(a). As can be seen there is approximately a $2 \%$ error in the maximum temperature (Fig. 45(b)). Further investigation using differently meshed structures (Fig. 46) shows that if the interface is finely meshed the difference between the Atar simulation and Atar-FCM becomes less than $0.2 \%$ and could be made arbitrarily small by further refinement. It should be noted that the Atar simulations also show a significant change as the mesh density is increased, this is due to the rapid spreading of the heat flow as it exits the mesa. This phenomena leads to "crowding" of the heat flow at the corners which is captured better by higher levels of discretization.

\subsubsection{FCM mesa correction}

Although the FCM mesa simulation can be brought into close agreement with the Atar results by finer meshing of the mesa interface, the root of the discrepancy can be seen in Fig. 47. The FCM represents the two materials as two separate clouds (one for each material region) with common interface nodes. At the interface there is 


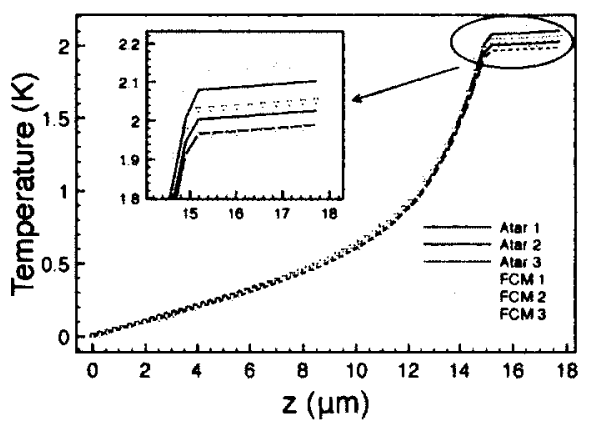

(a)

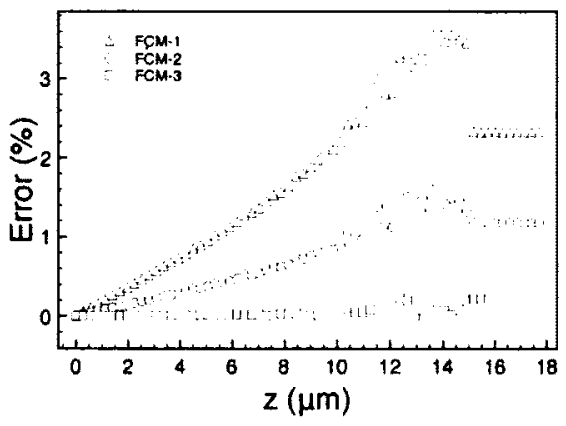

(b)

Figure 45: Mesa structure temperatures for both Atar and Atar-FCM. (a) Temperature along a vertical line through the centre of the mesa. (b) Temperature error, three different structures are plotted (see Fig. 46).

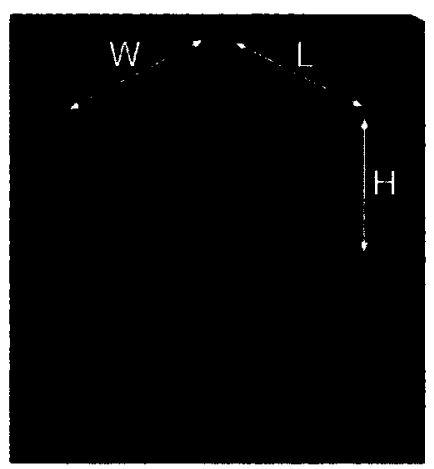

(a)

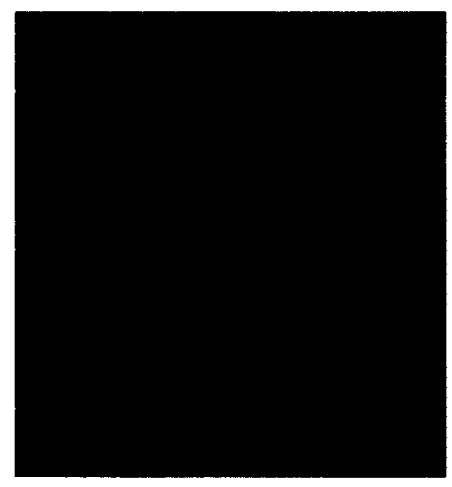

(b)

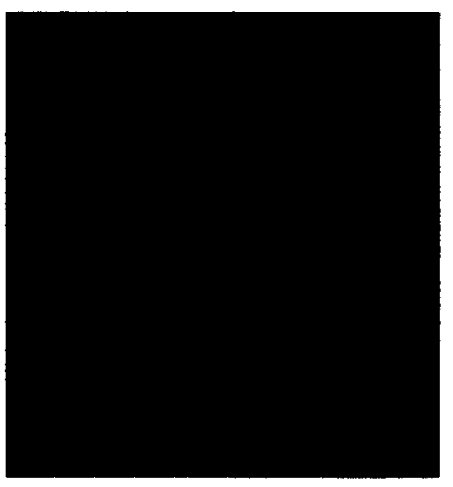

(c)

Figure 46: Atar mesh and Atar-FCM point distributions for a three sided mesa with three levels of refinement: (a) FCM-1. (b) FCM-2. (c) FCM-3.

an area $A$ associated with the heat flow normal to the interface for each cloud. The FCM, as described above, matches the gradient of the heat flows on either side of the interface, weighted by the appropriate thermal conductivities. For most of the interface nodes the two areas associated with the clouds are equal and the injected heat flows $\left(F=\kappa \nabla_{n} T \times A\right)$ are equal. However, for the corner node (shown in red), the structure of the two clouds (blue and green) result in different areas being present. The area associated with the substrate cloud (green) is larger than it should be and "extra" heat flow is injected into the substrate resulting in an erroneous rise in the 


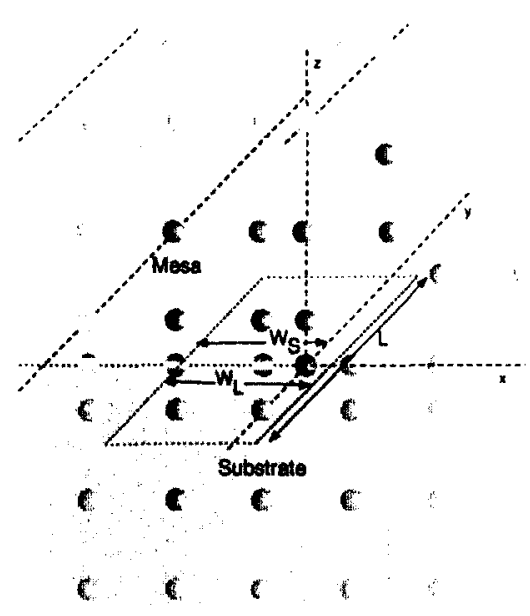

(a)

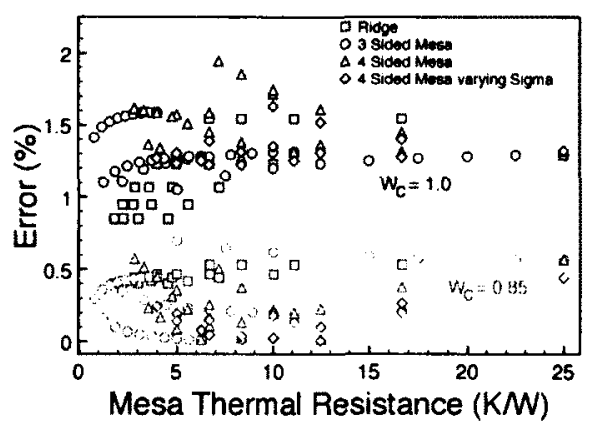

(b)

Figure 47: (a) Finite Cloud structure at Mesa Edge. The red node is the node of interest. Blue nodes are associated with the cloud for the mesa region. Green nodes are associated with the substrate cloud. Two nodes are shared at the interface. Yellow nodes are associated with neither cloud. (b) Temperature error versus mesa thermal resistance. The black symbols are the result of using an area weighting factor of 1.0 (the unadjusted case). The green symbols show the effect of using an area weighting factor of 0.85 giving an error below $0.6 \%$.

temperature distribution. This effect decreases as the point density at the interface is increased as the additional area is a smaller proportion of the total area of the bottom of the mesa.

It is straightforward when using the FCM to correct this error by scaling the area associated with the corner node by a weighting factor $W_{c}$. A simplistic analysis from Fig. 47(a) would suggest that the area should be scaled by 0.75 . However, the factor is complicated by the presence of a non-uniform heat flow across the interface at the corner. To determine an optimal value for the factor a large number of mesa structures were run with differing geometries ( 3 sided mesas, 4 sided mesas and ridges) and a widely varying mesa thermal conductivity $(1-1000 \mathrm{~W} / \mathrm{m}-\mathrm{K})$. It was found that the use of a factor of 0.85 was optimal and reduced the error for structures with mesh densities equivalent to the FCM-2 structure to $0.6 \%$ or less. In Fig. 47 (b) the error 
in the maximum temperature is plotted as a function of the mesa thermal resistance (see Fig. 46(a) and with $R_{t h}=H /(\kappa W L)$. This error could be further reduced by the use of finer meshing.

\subsubsection{Mesa transient analysis}

A very similar transient test to that of section 5.4 .3 has been repeated with the addition of a mesa structure on top, with the heat generation inside of the mesa structure. The structure nodes can be seen in Fig. 48(a). The transient response for a node at the center of the $x=0$ face of the main structure is shown in Fig. 48(b), comparing FCM results with Atar results. Again, we see a high degree of agreement between the two methods.

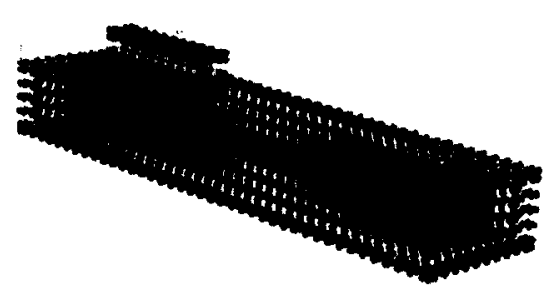

(a)

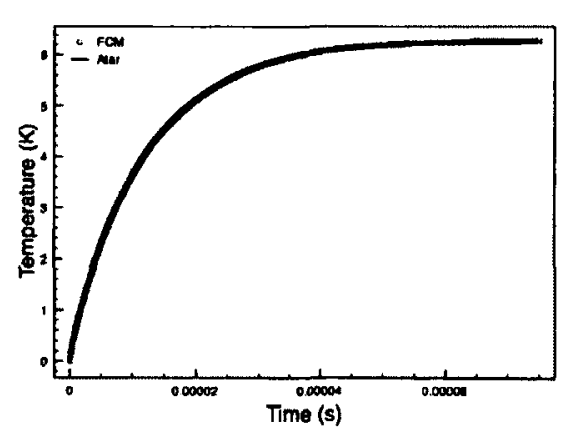

(b)

Figure 48: (a) Node placement for the simple transient mesa analysis with pink nodes the adiabatic boundary points, red being the Dirichlet nodes, grey the internal nodes, and yellow nodes are identified as the heat generating points. (b) Transient response for both the Atar solution and the FCM solution. 


\subsection{Using FCM thermal models for simulation and TCAD}

In the previous section it was shown that accurate FCM thermal models could be built. In this section the use of the FCM will be investigated for building complex optical models of integrated optical components. This will show the potential for combining the FCM with solution and model building methods, to address an important future TCAD issue; the creation of small efficient thermal compact models. This issue is important to the continued development of highly "integrated" mixed optical and electrical circuits which will be dependent on the establishment of a Computer Aided Design (CAD) environment with a degree of sophistication comparable to current electrical design tools [30]. The establishment of this infrastructure is challenging due to coupling between a number of physical domains and the disparate nature of the current tools used in each domain. In particular, detailed transient simulations of moderate sized circuits (10-1000 devices) such as is undertaken in Spice-like electrical simulators $[31]$ is not well developed. A primary requirement for the effective use of such simulators for electro-optic circuits is the development of small compact physically based device models for optical components with accurate thermal characteristics $[32,33]$.

The creation of such models is intrinsically challenging due to a number of factors: the three dimensional nature of the heat flow, the presence of distributed heat sources within large optical devices, thermal coupling, and non-linear device behaviour. For optical circuits this is further complicated by the thermo-optic effect (the variation of the optical index with temperature), which is strong enough in most planar optical devices to make them sensitive to small temperature changes and is particularly important in devices based on interference effects. In the following section I will show 
(a)

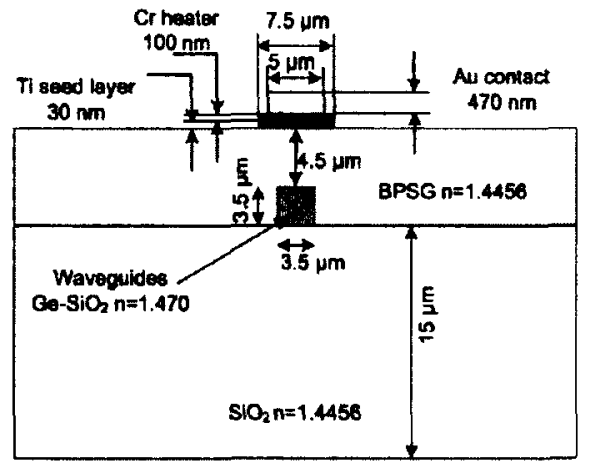

(b)

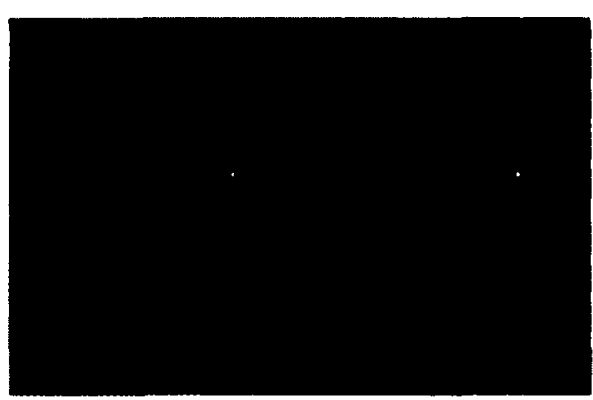

(c)

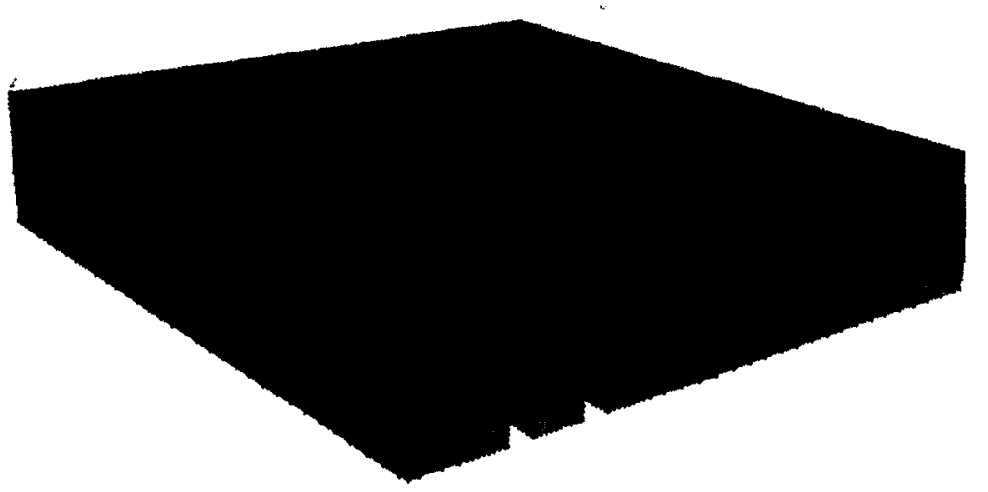

Figure 49: Silica based Mach-Zehnder Device. (a) Cross-section of optical waveguide with heater element. (b) Layout of two semi-circular $\mathrm{MZ}$ with heaters placed on the outer arms. (c) Atar model of $1 / 2$ of a MZ element with additional thermal backside via $[3,4]$.

for four devices, built in two different technologies, how these models can be built and then used in an optical circuit simulator. The first device will be a thermally configurable Mach-Zehnder (MZ) device built in a silica-based technology. The other three devices will be based on a Silicon-on-Insulator (SOI) technology.

\subsubsection{Silica based Mach-Zehnder model}

The first large optical model is that of a semi-circular MZ with an integrated heater. Fabricated in a silica based optical waveguide technology, the device has been used in a number of technologies including: pulse repetition circuits and microwave photonics 
applications $[3,4]$. The technology consists of an optical waveguide fabricated in silica-on-silicon. Semi-circular MZ interferometers are fabricated with a built in $\mathrm{Cr}$ heater in the outer arm (see Fig. 49(a)). A layout for a typical device is shown in Fig. 49(b). The heater is used to thermally tune the MZ by exploiting the thermal dependence of the index of refraction. The devices are large with arm lengths of $6.28 \mathrm{~mm}$ and $8 \mathrm{~mm}$. To model the devices optically a detailed thermal map of the outer waveguide is needed requiring a large complex $3 \mathrm{D}$ model of the heat flow in the device. Applications utilizing the device exploit the interference of the two optical signals passing through each arm to create optical impulse responses. A key factor to be modelled is the variation in the index along the waveguide as a function of temperature. To create a compact model for use in a circuit level simulator a model is needed that can provide a temperature distribution along the thermally activated arm.

In Fig. 49(c) an Atar-FCM model of an MZ element is shown. This model was used as an example of how the FCM could be exploited to build a large complex model in Sec. 4.2.4. The model consists of $1 / 2$ of the MZ element (as symmetry can be exploited) and an integrated backside thermal via for active cooling. The complete model is very large consisting of 865,683 nodes. The primary difficulty this model presents is the large number of nodes and the need to know the temperature along the length of the heated $\mathrm{MZ}$ arm in order to determine the optical operation of the device.

\subsubsection{SOI based optical devices}

The second technology considered is a Silicon on Insulator (SOI) platform with a $1 \mu \mathrm{m}$ buried oxide (BOX) layer. Three models will be built: 1) A micro-ring based modulator; 2) a thermally configurable micro-ring switch and 3) a micro-disc laser [34-37]. 
(a)

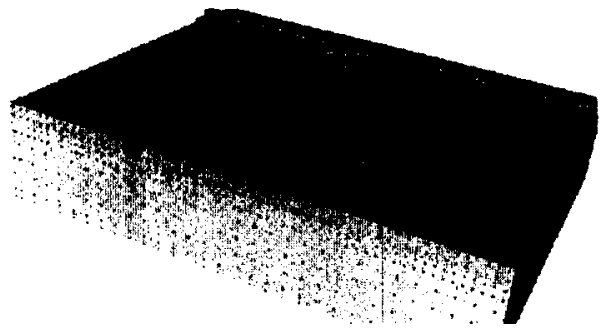

(c)

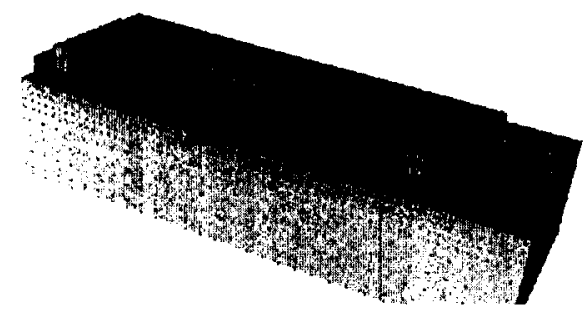

(b)

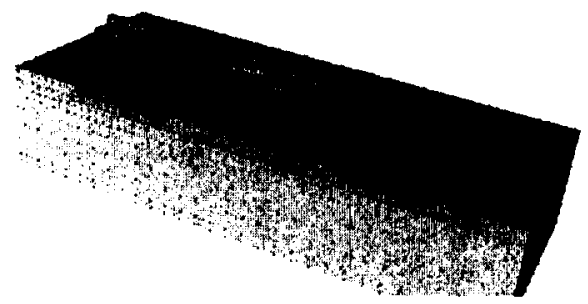

(d)

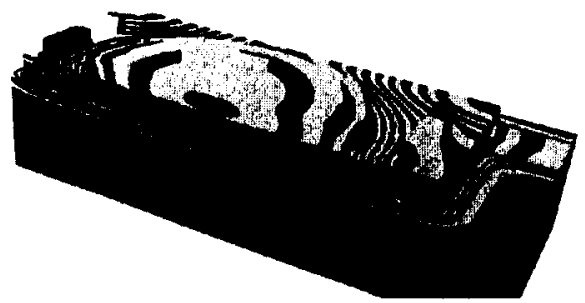

Figure 50: Models built using FCM and Atar. (a) Optical ring based modulator with depletion modulation and thermal tuning. (b) Cross-section of ring based optical switch. (c) Cross-section of InP Disc laser. (d) Thermal contours for a standalone simulation of the disc laser model.

Although many integrated optics platforms and devices have been explored, SOI based technologies are very attractive for silicon-based integration $[38,39]$. These devices were simply chosen as characteristic of proposed integration schemes. Waveguides within the devices consist of a first Si layer with a thickness of $250 \mathrm{~nm}$ and a ridge of $500 \mathrm{~nm}$. The waveguide ridge has width of $500 \mathrm{~nm}$. To build the models, Atar is used initially to build a block based model of the structure omitting any curvilinear pieces. This provides an easy method of building the substrate, waveguides and contact structures with an effective non-uniform mesh. Secondly, curvilinear elements are added into the model. This step is facilitated greatly by the FCM which allows for an unstructured mesh. When merging a new piece of the model, previous nodes in the volume are simply removed and the new nodes added to the model.

The first model is an optical modulator based on a micro-ring device (Fig. 50(a)). An $8 \mu \mathrm{m}$ radius ring is placed next to a straight waveguide producing a high $\mathrm{Q}$ resonant structure. Two sets of contacts are used to alter the resonant conditions of 
the ring. The thickness of the metal contacts used for the modulator are $0.5 \mu \mathrm{m}$. On the right side of the ring the one third of the ring closest to the guide is used as a "heater" to thermally tune the ring. An electrical current is passed through the ring from the interior contact to the exterior producing ohmic heating and causing the index to change due to the thermo-optic effect. This change results in a shift of the optical length of the ring and the resonant frequency. Due to the thermal capacitance of the device this is a relatively slow process and is used to tune or configure the ring. The second set of contacts is placed on the remaining two thirds of the ring and is used to electrically modulate the ring index. This is achieved by forming a pn diode in the ring and using carrier depletion to exploit an electro-optic effect [40]. This modulation of the ring can be done very quickly and used to impress a bit-stream on light travelling through the straight waveguide. In this structure the metal contacts were placed 0.25 microns from the waveguide ridge. Optical mode simulations showed that the quasi-TM and TE modes were tightly constrained to the waveguide and had losses of $14.4 \mathrm{~dB} / \mathrm{cm}$ and $130.9 \mathrm{~dB} / \mathrm{cm}$ respectively. These losses would need to be considered and optimized for a working device.

The thermal model of the modulator has two sources of heat. The primary source of heat is the ohmic heating of the guide due to the "heater". This heat source is modelled as a volume of uniform generation in the base of the ring between the two contacts. The second source is ohmic heating created in the electronic modulator due to the need to charge and discharge the pn junction [34]. This source is dependent on the transient state of the modulator, but is typically small compared to the other source.

The second device is a micro-ring based switch shown in cross-section in Fig. 50(b). Similar to the modulator but with only a simple heater present on the left side of the device it has a second waveguide present on the right side. During operation 
Table 3: Material thermal properties.

\begin{tabular}{c|ccccccc} 
& Gold & Copper & $\mathrm{InP}$ & Polymer & $\mathrm{SiO}_{2}$ & $\mathrm{Si}$ & $\mathrm{Cr}$ \\
\hline$\kappa(\mathrm{W} / \mathrm{m}-\mathrm{K})$ & 319 & 400 & 68 & 0.5 & 1.3 & 130 & 93 \\
$c\left(\mathrm{~J} / \mathrm{K}-\mathrm{cm}^{3}\right)$ & 2.49 & 3.42 & 1.50 & 0.22 & 2.26 & 1.63 & 5.01
\end{tabular}

the heater is used to switch the ring in and out of resonance using the thermo-optic effect. When the ring is off-resonance light incident from the left will be unaffected by the presence of the ring and will continue along the waveguide running left to right. However, at resonance the light incident from the left will be transferred to the second waveguide and blocked from travelling past the ring. Within this model there is a single heat source due to ohmic heating between the heater contacts. This heat source was modelled as a rectangular region in the base region of the Si guide.

The final model is a micro-disc laser based on whispering gallery modes $[36,37]$. The disc itself is fabricated in InP with a central metal contact and external contacts on an $\mathrm{InP}$ contact layer. The InP structure is integrated onto the SOI substrate using a polymer buffer layer and the light coupled vertically into a waveguide fabricated in the SOI platform. The thermal generation region of the laser was assumed to be defined by the disc geometry. The layer thicknesses of the polymer was $1.25 \mu \mathrm{m}$ and the InP disc $0.75 \mu \mathrm{m}$. The thickness of the metal contact used for the top contact was $0.25 \mu \mathrm{m}$.

All of these devices are somewhat speculative in nature (in particular the disc based laser), as is the integration technology; however, the intent here is to show the potential for thermally modelling geometrically complex structures, linking them together and incorporating these models into a circuit level simulator.

The material thermal properties used in all the models are given in Table 3. 


\subsubsection{Model reduction compact modelling}

The primary difficulty in the use of the complex 3D models described above is that the matrices used to define the heat flow equation, (87), will be large, sparse, and very cumbersome to link to a device or circuit level simulator. However, previous work has shown that model reduction (MR) methods based on Krylov subspace techniques can be used to convert these equations to a more useful compact form [41]. This compact form of the problem allows both for quick simulation of the models and the incorporation of the model into a circuit level simulator.

The first step of the algorithm is to compute the multidimensional moments of the system in (87). For that purpose, the block moments with respect to frequency are computed using the procedure described in [42] exploiting Krylov subspace techniques. Block moments of $\mathbf{T}$ with respect to all source vectors are computed using the technique elaborated in [43]. More details on this procedure can be found at [42-44].

Using the technique briefly outlined above we obtain a congruent matrix $\mathbf{Q}$ which can be used to transform (87) to give,

$$
\overline{\mathbf{C}} \frac{\partial \overline{\mathbf{T}}}{\partial t}=\overline{\mathbf{G}} \overline{\mathbf{T}}+\overline{\mathbf{B}}+\sum_{j=1}^{m} \overline{\mathbf{R}}(\mathbf{t})
$$

where,

$$
\mathbf{T}=\mathbf{Q} \overline{\mathbf{T}}
$$

and the reduced matrices and vectors are obtained using congruent transforms such 
as,

$$
\begin{aligned}
& \overline{\mathbf{G}}=\mathbf{Q}^{\mathrm{T}} \mathbf{G Q} \\
& \overline{\mathbf{B}}=\mathbf{Q}^{\mathrm{T}} \mathbf{B}
\end{aligned}
$$

where $\mathbf{Q}^{\mathbf{T}}$ is the transpose of $\mathbf{Q}$. The size of $\mathbf{Q}$ is determined by the number of moments taken during its creation.

The primary computational cost of creating the reduced system is a single $\mathrm{LU}$ decomposition of the $\mathbf{G}$ matrix in (87). The system of equations obtained this way is much smaller than the original one, albeit dense. Reductions of systems from sparse systems of order of $10^{5}$ unknowns to 30 unknowns are typical with only very small errors of less than $1 \%$ [41]. Typically six frequency moments are needed with additional moments needed for each source vector.

\subsubsection{Multiple models and linking}

The previous sections dealt with a single model composed of multiple regions. It is often desirable and sometimes necessary to build complex multi-component structures out of individual models. The coupling between models with common interfaces is achieved using thermal ports. These ports are incorporated into the model equations (87) by the incorporation of port heat flows giving,

$$
\mathbf{C} \frac{\partial \mathbf{T}}{\partial t}=\mathbf{G} \mathbf{T}+\mathbf{B}+\sum_{j=1}^{m} \mathbf{R}_{\mathbf{j}}(\mathbf{t})+\mathbf{L}_{\mathbf{p}} \mathbf{F}_{\mathbf{p}}
$$

where $\mathbf{L}_{\mathbf{p}}$ is a linking matrix that "applies" the heat flows $\left(\mathbf{F}_{\mathbf{p}}\right)$ from connecting models. The matrix $\mathbf{L}_{\mathbf{p}}$ has the dimensions of $n_{p} \times n_{m}$ where $n_{p}$ is the number of ports and $n_{m}$ the number of unknowns in the original model. The configuration of 
the ports can be defined in a variety of ways [45-47]. If every node present on the common interface is designated as a port then the number of ports is typically very large and the resultant system is essentially identical to a structure built as a single model. We designate this type of simulation as a "full" simulation. Although creating a "full" model from a set of connected models has some advantages when building the structure, simulation or reduction of the global matrices is identical in computational cost to building and solving a single model.

Alternatively, we can designate geometrically defined "ports" encompassing multiple boundary nodes but defined by a common port temperature and heat flow. This obviously introduces some error into the model, but reduces the number of ports dramatically. The error introduced can typically be well controlled by a judicious choice of port definitions.

The motivation to reduce the number of ports can be seen when we apply MR to (99) giving,

$$
\overline{\mathbf{C}} \frac{\partial \overline{\mathbf{T}}}{\partial t}=\overline{\mathbf{G}} \overline{\mathbf{T}}+\overline{\mathbf{B}}+\sum_{j=1}^{m} \overline{\mathbf{R}}(\mathbf{t})+\overline{\mathbf{L}}_{\mathbf{p}} \mathbf{F}_{\mathbf{p}},
$$

where $\overline{\mathbf{L}}_{\mathbf{p}}$ is a reduced linking matrix which has the dimensions of $n_{p} \times n_{r}$, where $n_{r}$ is the size of the reduced system. It can be seen in the formation of this equation that each port in the original system is, in essence, an independent source vector. Therefore in the formation of the reduced system moments need to be taken with respect to each column of $\mathbf{L}_{\mathbf{p}}$; this implies that the size of reduced system will be strongly determined by the number of ports.

It is crucial to keep the size of the reduced system small and the use of "common" ports is essential for the creation of useful reduced models. The use of model reduction and linked models is very powerful. The cost of creating the reduced systems is much smaller if done individually for each model - as the LU decomposition cost is 
geometric with the number of unknowns. Once created the reduced models can be linked together into a small global system, a solution obtained and all the original temperatures recovered by the use of $(97)$.

These small reduced models are ideal for optical thermal compact models for circuit level simulation. They are composed of relatively small linear models described by dense matrices of dimensions less than 100 and very accurately capture transient behaviour. Boundary condition dependancies can be linked together to form larger models and provide quick access to all the original temperatures in the detailed models.

\subsubsection{Individual model simulation and reduction}

This section will present results for the verification of the use of model reduction on the four Atar-FCM optical models described above. The CPU time and memory needed to perform a model reduction is roughly equivalent to the full simulation as the primary task for both is an LU decomposition. The time and memory needed to simulate the reduced model and obtain the full temperatures once the reduction is done is negligible as all that is involved is a solution of a small dense matrix (40-150 unknowns) and a matrix multiplication to extract the original temperatures. This adds significant computational savings over the need to repeat a full simulation for each different parameter. Solutions undertaken in this section were obtained using $M A T L A B[48]$ to provide a readily available computational baseline. All runs were done on a 64 bit Intel based PC with 12 cores running at $3.33 \mathrm{GHz}$ and 120 Gigabytes of RAM.

\section{Silica-based Mach-Zehnder model}

For this model the source of heat was the $\mathrm{Cr}$ heater along the top of the waveguide. 


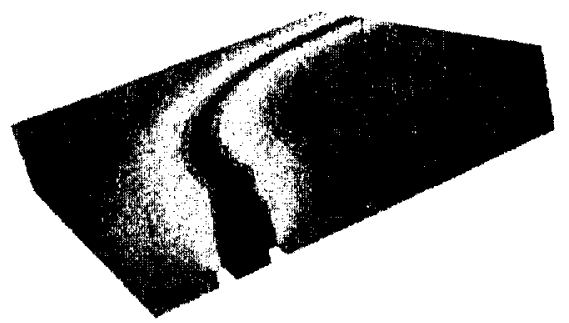

(a)

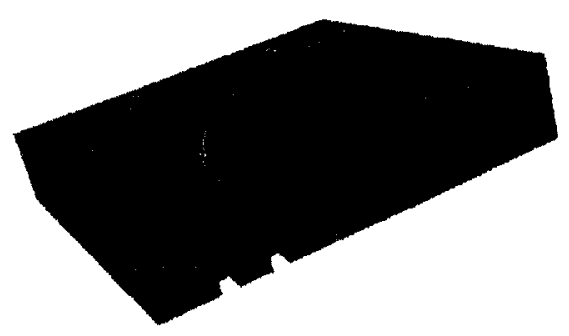

(c)

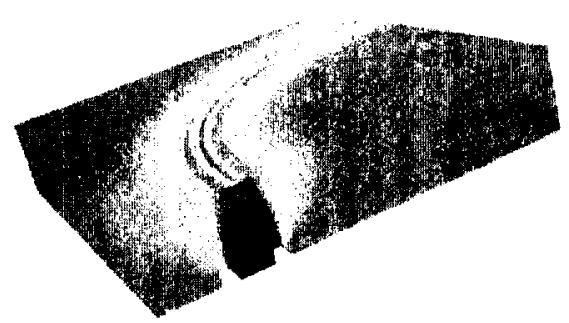

(b)

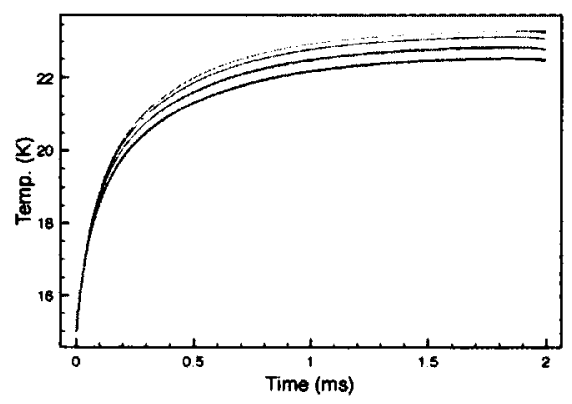

(d)

Figure 51: Thermal contours for circular waveguide. Active cooling of thermal via. (a) No power in heater. (b) Powered heater. (c) No cooling of thermal via but with power in the heater. (d) Thermal transient for heater power transitioning from off to on (11 points distributed along arc). 
Two fixed boundary conditions were placed on the bottom of the device. One for the bulk of the device and a second on the thermal via contact directly under the waveguide. The other sides were set to be adiabatic. The creation of the reduced model was found to take $2144 \mathrm{~s}$ and the size of the reduced model was 140 . Using this reduced model, simulations were undertaken for a variety of boundary conditions (see Fig. 51) and a comparison to a full model solution showed that the temperatures were accurate to within $0.1 \%$. A single steady-state simulation of the model takes $708 \mathrm{~s}$ for a full model and roughly $10^{-3} \mathrm{~s}$ for the reduced model. Therefore given an initial investment to create the reduced model, simulations can be undertaken for a variety of conditions quickly and efficiently. It is also possible to perform a transient simulation using the reduced model (Fig. 51(d)) which, of course, is also much quicker than a full model simulation.

\section{SOI based models}

The SOI based devices shown in Fig. 50(a-c) were simulated individually using the internal power sources present. Adiabatic boundary conditions were present on all sides except the bottom for which $\mathrm{T}$ is set to zero. A characteristic contour plot is presented for a cross-section of the laser disc model in Fig. 50(d). The combination of thermal isolation due to the BOX and polymer layers, the distributed heat generation in the disc and the relatively high thermal conductivity of the waveguides produces a complex heat flow. Due to the complexity of the geometry and heat flow a large number of nodes $(\sim 300,000)$ are needed to represent each model as presented in Tab. 4. Initially, full simulations of all three models were performed (without the curvilinear elements) and temperature profiles were compared to Atar simulations showing good agreement. The full models with curvilinear elements were also determined to conserve heat flow to within a few percent. Due to the size of the models 
Table 4: Model data: $S_{f}$ - full model size; $S_{r}$ - reduced model size; $T_{f}$ - cpu time for full simulation; $T_{r e d}-$ cpu time to perform a model reduction; $T_{r s}-$ cpu time to simulate a reduced model; $\mathrm{M}$ - Memory required for full simulation/model reduction; All times in seconds. Memory figures in gigabytes. Model size is number of unknowns. A $\sim$ indicates the number was negligible. Solutions were obtained using MATLAB on a 64 bit Intel based PC with 12 cores running at $3.33 \mathrm{GHz}$ and 120 gigabytes of RAM.

\begin{tabular}{l|cc|ccc|c} 
Model & $S_{f}$ & $S_{r}$ & $T_{f}$ & $T_{\text {red }}$ & $T_{r s}$ & $\mathrm{M}(\mathrm{GB})$ \\
\hline Modulator & $295 \mathrm{~K}$ & 82 & 164 & 406 & $\sim$ & 15 \\
Ring Switch & $270 \mathrm{~K}$ & 42 & 90 & 158 & $\sim$ & 12 \\
Disc Laser & $207 \mathrm{~K}$ & 42 & 104 & 170 & $\sim$ & 10 \\
\hline Linked & $672 \mathrm{~K}$ & 318 & 1154 & N/A & 0.04 & 50
\end{tabular}

simulation times were quite long (roughly 2 minutes) and memory usage was large (greater than $10 \mathrm{~GB}$ ). The temperature profiles obtained from the reduced models were found to be essentially identical to the original system with errors of the order of 1 part in 10,000. It should be noted that multiple simulations for differing power levels and boundary conditions can be done with a single reduced system.

\subsubsection{Linked model simulation}

To illustrate the use of model linking the three SOI based models described above were linked together as shown in Fig. 52(a). The models were linked in two ways: 1) a fully linked boundary at which each boundary node was matched to an adjacent one in the opposite model (a "full model"), or 2) ports were defined on the interface of the two models over which there is common port temperature and heat flow. For both interfaces, 12 ports $(6 \times 2)$ were used for the $\mathrm{Si}$ substrate, 6 ports for the $\mathrm{BOX}$ region $(6 \times 1)$ and 1 port for the waveguide for a total of 19 ports per interface. When forming a linked model using defined ports the reduced models of the devices were used. 
(a)

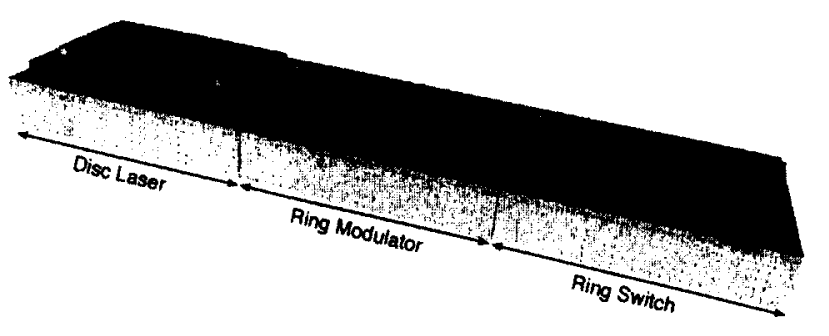

(b)

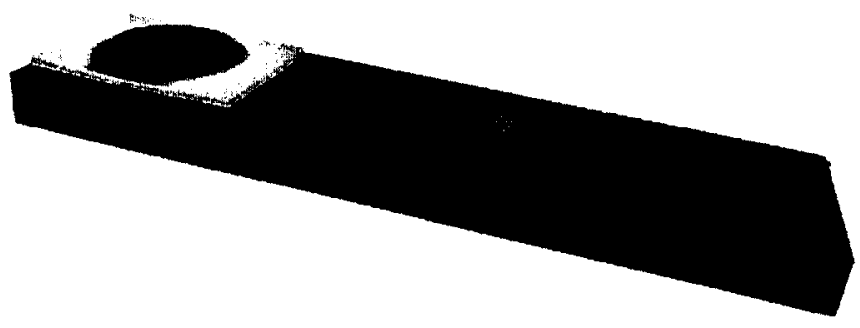

Figure 52: Linked model simulation. (a) Complete linked model with all three devices. (b) Temperature profile for linked model.

The simulation time for a full linked model was very large (1154 sec.) due its size of 672,000 nodes, and it required a very large amount of RAM - in excess of $50 \mathrm{~GB}$ (see Tab. 4). The linked reduced model was formed from the individual reduced models and the simulation time $\left(t_{r s}=0.04 \mathrm{sec}\right)$ and memory requirements were very small as the total reduced system size was 318 unknowns. The speedup from using the model reduction was 28,000 times. Although this is, of course, disregarding the one time cost of building the reduced model.

A contour plot of the temperature distribution in the linked structure is shown in Fig. 52(b). The maximum temperature in the laser was $45.2 \mathrm{~K}$ for a total laser power dissipation of $7.5 \mathrm{~mW}$. A boundary condition of $\mathrm{T}=0$ was placed on the bottom of the structure. Local heating of the modulator and ring switch can be seen and was due to biases placed on the device to tune them for optical operation. Details of this will follow in the next section.

Of significant interest for optical design is the temperature profile around the two rings. This is plotted in Fig. 53(a) and shows that the ring switch is thermally 
(a)
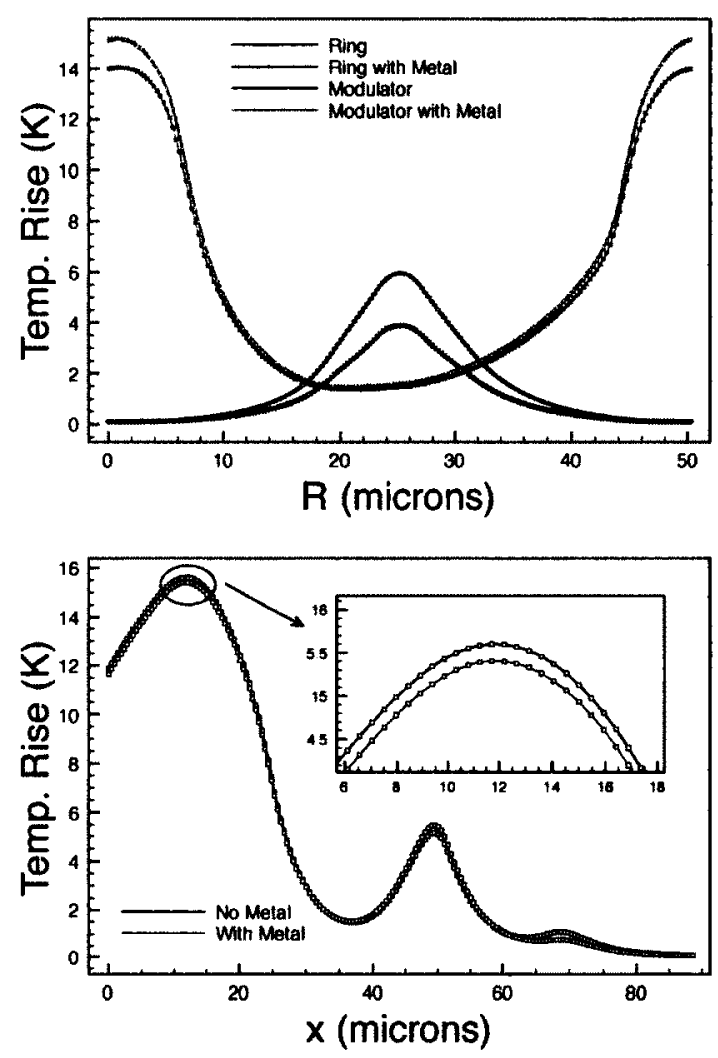

(b)

Figure 53: Temperatures in models. (a) Temperature rise around the circular waveguide in the Ring Switch and the Modulator ( $R=0$ is on the left hand side of the two structures and increases counter-clockwise). (b) Temperature rise along the straight waveguide section. Full simulation results are shown as solid lines; reduced simulation results as symbols. Both models with and without metalization are shown.

biased to maximum of $4.0 \mathrm{~K}$ and the modulator to $15.3 \mathrm{~K}$. A second plot shows the temperature profile along the straight waveguide running in the $\mathrm{x}$ direction. As expected there is a large temperature rise underneath the laser with peaks also present at the intersections with the two other devices. It is evident from this plot that the $\mathrm{Si}$ waveguide is a source of thermal coupling between the devices. In both of these plots the full simulation is shown as a solid line and the simulation based on reduced models as symbols. As can be seen there is no evidence of any appreciable error due to either the model reduction or the use of defined ports at the interfaces of the 
(a)

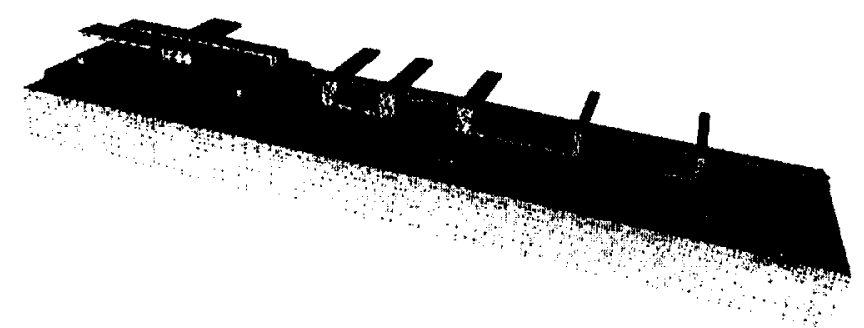

(b)

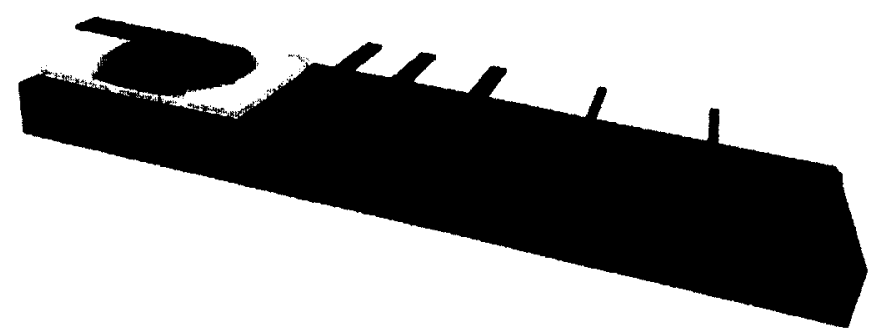

Figure 54: Linked model simulation with a more complete metalization. (a) Complete linked model with all three devices. (b) Temperature profile for linked model.

models. The error for linked models was found not to exceed $1 \%$.

It is well known that for SOI structures the metalization of the circuit can provide an important path for thermal coupling between devices. The SOI technology introduces a large degree of thermal isolation to the substrate as can be seen in the contours in Figs. 50(d) and 52(b). Because of this the thermal impacts of layout and routing of the metalization can have large impacts on circuit design. To illustrate this a second set of models were built with a more extensive metalization structure. These models are shown in Fig. 54(a). For this model vias were added and 1st level metal incorporated (which had a thickness of $0.5 \mu \mathrm{m}$ ). In particular a common ground strap was added between the heaters of the modulator and the ring switch (an extra port was defined to accommodate this feature). The contour plot for this structure is shown in the second figure and the ring and guide temperatures plotted in Fig. 53(a) and (b). As can be seen in these figures the effect of the additional metalization is significant with a slight cooling of the laser due to heat spreading but 
most importantly a large degree of coupling is now present between the modulator and the ring. The modulator has become significantly cooler and the ring warmer. The optical implications of this will be discussed in the next section.

\subsubsection{Integrated simulations}

The sections above have shown that the reduced FCM models are accurate, small, and capable of capturing the entire temperature distribution of the initial detailed models. For the purely thermal simulations performed above the use of model reduction is very useful. The one time cost of building the reduced model (which takes somewhat longer than a single simulation) can be invested to obtain the ability to do multiple simulations with different boundary conditions and power levels very quickly. The ability to build a library of models that can be linked and simulated is very attractive, particularly as process tool kits and device libraries become more common $[49,50]$. However, perhaps the most attractive use of the reduced models is as a compact model for circuit or system level simulation.

A circuit or system level simulator will have a global set of equations that need to be solved. A variety of approaches can be used. However, in all them the thermal power generation will be supplied to the thermal model and a set of the calculated temperatures used to update device equations. For the case of Spice-like simulators a set of global matrices (representing a set of 1st order algebraic-differential equations) are created by the circuit simulator into which devices/models are "stamped" [31]. These matrices (plus two vectors - one time dependent and the other representing non-linearities) are then used for time-domain simulation of the circuit. Within this framework the reduced thermal models are very naturally incorporated into the matrices. Each model consists of small dense matrices representing capacitance and thermal conductance (in the reduced variable space). These matrices are simply 
"stamped" into global matrices. Thermal power from the devices is introduced into the model through the global non-linear vector. The only complication is that the temperatures needed for the device equations must be obtained from the reduced variables. This is easily done by introducing new variables to represent these temperatures and "stamping" in the appropriate values (obtained from the $Q$ matrix) into the conductance matrices. This method of incorporating thermal models described by matrices can be done with either the original sparse matrices or the small reduced matrices. However, the use of the very large original matrices will likely overwhelm the circuit simulator and make the problem intractable.

To demonstrate the effectiveness of the reduced models, the opto-electronic circuit simulator OptiSPICE $[32,33]$ will be used. This simulator is based on a spice-like architecture; defining a complex optical signal, physically based compact optical models and a multi-channel multi-mode architecture. Details on the models used for the simulation and the methodology can be found in [32] and [33]. This simulator (marketed by Optiwave Inc [51]) was purely used for convenience, any similar simulator could be modified to incorporate the linear reduced models.

The schematic of the circuit to be simulated is shown in Fig. 55. To simulate this circuit in OptiSPICE (as shown in Fig. 55) a matrix-based thermal model (either full or reduced) is incorporated in to the circuit and the thermal power associated with each circuit element linked to a power source in the thermal model. Likewise, temperatures from the thermal model can be connected to optical and electrical devices. To model the ring elements with a varying temperature the circular waveguides were broken up into a number of waveguides each with a unique temperature obtained from the thermal model. For the simulations undertaken here it was found sufficient to use 8 waveguide elements for both devices.

For all of the integrated simulations the reduced thermal models were used. It 


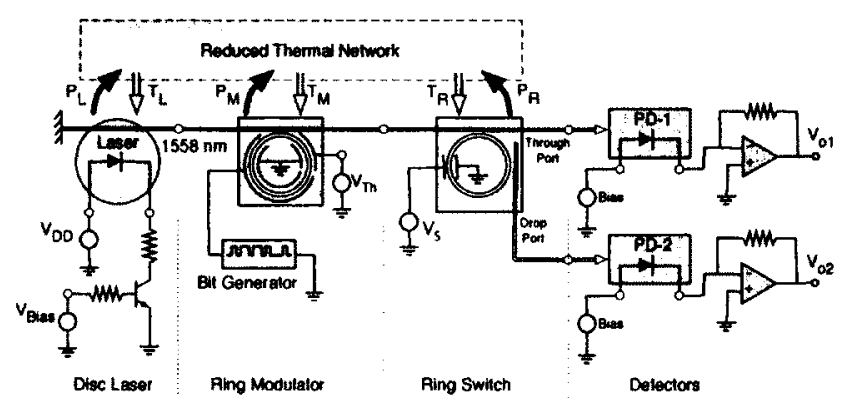

Figure 55: Schematic of simulated system comprised of a laser, modulator, switch and two detectors and a distributed thermal model.

was attempted to link full models for comparison, however, even on a 12 core Intel based machine with $120 \mathrm{~GB}$ of RAM this proved unworkable. In contrast, the use of the reduced thermal model enabled simulations to be completed in less than 1 minute on a 2 core $4 \mathrm{~GB}$ machine. Full model thermal transients were therefore run independently to provide for a comparison to the reduced simulation obtained from the integrated simulations. For these full simulations power levels were obtained from the OptiSPICE simulations and used as an input.

Initially, simulations were undertaken on individual elements of the circuit. The two elements looked at were the laser model heating during an initial power up transient and the ring switch transient response during a transition from resonance to off-resonance. The laser was assumed to be $5 \%$ efficient [36] generating $7.5 \mathrm{~mW}$ of thermal power in order to produce a constant wave single sided optical power of $0.375 \mathrm{~mW}$ leading to maximum temperature rise of $35 \mathrm{~K}$.

Optical mode simulations of the waveguides determined that the $n_{\text {eff }}$ of the silicon waveguides was 3.1 and the ring based switch was thermally tuned to resonate at $1558 \mathrm{~nm}$ using an application of $0.26 \mathrm{~mW}$ into the heater producing the thermal distribution shown in Fig. 53(a) and an average temperature of $1.85 \mathrm{~K}$. When the power to the ring is increased and the temperature raised by $30 \mathrm{~K}$ on average the ring 


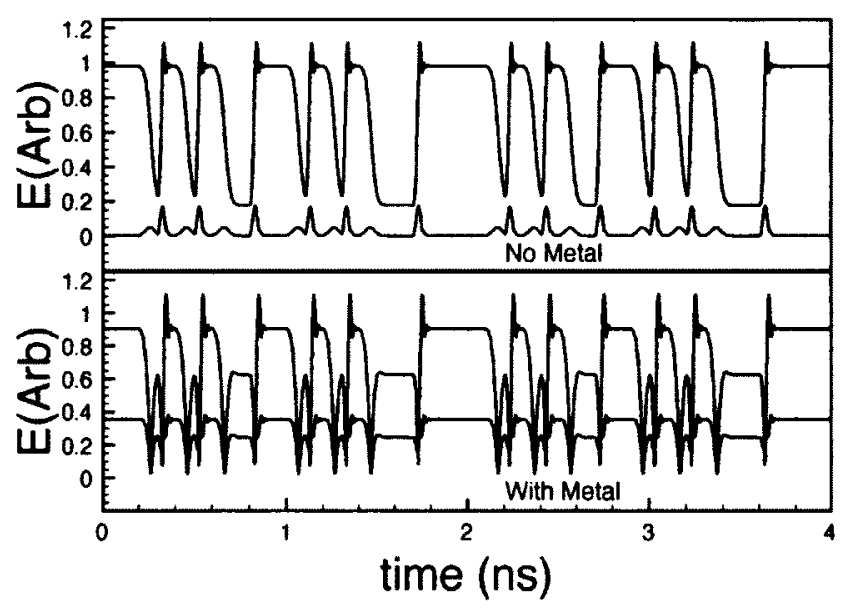

Figure 56: System response. System is tuned to pass the data to the drop port. The drop port response is shown in black and the through port response in red. Top plot shows simulation using temperature from the nominal model. Bottom plot presents a simulation for temperatures from the model with metalization present.

is switched to off resonance so that the drop port is now the inactive port. The shift of the ring resonant wavelength is $1.5 \mathrm{~nm}$ or equivalently $188.66 \mathrm{GHz}$.

The final stage of circuit level simulation was to perform a full transient simulation of the circuit with an applied bit stream modulating the laser output. A bit stream was applied to the modulator with a voltage swing of $2.5 \mathrm{~V}$. Individual bits had rise/fall times of $40 \mathrm{ps}$ and total length of $200 \mathrm{ps}$. The modulator was thermally tuned using the application of $0.75 \mathrm{~mW}$ and average temperature rise of $7 \mathrm{~K}$. This moved the ring off-resonance to allow transmission with a small loss of $0.35 \mathrm{~dB}$ at $1558 \mathrm{~nm}$. The voltage swing was sufficient to restore resonance in the ring and block transmission through the modulator by more than $15 \mathrm{~dB}$. The result of this simulation for the nominal structure is shown in Fig. 56 in the upper panel. This figure presents the output on both output ports of the ring switch. The ring is thermally biased so that the active port is the drop port and the bit stream appears on this port (shown in black). As can be seen the modulator has produced some ringing in the bits which 
is characteristic of ring based modulators. The output on the through port (shown in red) is essentially the high frequency content of the input from the modulator that fall outside the ring filter response and is suppressed by at least $15 \mathrm{~dB}$. The second panel in this figure shows the effect of the additional metalization present in the second linked structure (Fig. 54). The perturbation of temperatures of the ring and the modulator has produced a very inferior separation of the input signal. The lowering of the modulator temperature has produced an over-modulation of the laser source by the applied voltage. The raising of the ring temperature has de-tuned it from $1558 \mathrm{~nm}$ and results in a much larger portion of the optical power being present on the through port and only a $9 \mathrm{~dB}$ suppression of the bit stream.

\subsection{Summary}

In this chapter the process to solve materially inhomogeneous problems is detailed and a simple example given to show the process. The method is then tested with simple time independent heat diffusion models, all the way to complex three dimensional transient problems. Results from these tests show that the FCM gives accurate results when compared with either analytical solutions or results from Atar, a finite difference PDE solver.

This use of Atar highlights the ease with which the FCM is able to piece together various shapes without significant concern for point distributions or a need for remeshing. As Atar has an advanced model geometry generating tool, it is possible to create the rectilinear pieces using this tool, and simply add in the curvilinear pieces.

The method is then attempted with much larger more complex opto-electric problems, for which model order reduction is necessary, or at least very helpful, in finding a solution. It is shown that MR can be quite useful with the FCM to reduce large 
models and to reduce split sections of larger models which are then linked together. 


\section{Chapter 6}

\section{Wave equations}

The finite cloud method has thus far been successfully applied to simple Laplace and Poisson type problems and the thermal diffusion equation. The following chapter investigates several wave equations including Schrodinger's equation and Maxwell's equations. These equations have several nuances which have not yet been examined and are more fully explored in this chapter.

\subsection{Scalar wave equation}

The base equation studied is the scalar wave equation which is a hyperbolic partial differential equation,

$$
\nabla^{2} u=C \frac{\partial^{2} u}{\partial t^{2}}
$$

which describes the motion of a wave propagating within a medium, with $u$, the field variable to be solved, being the nodal displacement, and the speed of the wave in a given medium is denoted by $C$. As this is a materially homogeneous equation, one must ensure that at material junctions the correct properties are being enforced. The precise condition on the interface between regions of differing material properties is 
dependent on the wave equation being solved, and will ensure conservation of the flow of a physical quantity (such as energy) across the junction.

\subsubsection{Time stepping in wave equations}

Of particular interest is the requirement of a new time stepping method in order to solve a hyperbolic PDE. As previously mentioned, the time stepping or integration formulae have regions of stability which can restrict the size of time step allowed. Having a time step $\Delta t$ which falls outside of the region of stability can cause the solution to oscillate and diverge. The backward Euler method which proved highly effective with the heat diffusion equation, due to its region of stability encompassing the entire left half plane, also has a stability region which extends into the right half plane.

Due to the nature of hyperbolic PDEs, any integration or time stepping method with a region of stability which extends into the right half plane will lead to unwanted damping of the solution. Two integration methods which are appropriate for hyperbolic PDEs are the forward Euler method and the trapezoidal method. Both of these methods will be used in this chapter and are now explained [6].

The forward Euler method is given as

$$
u_{t}^{n}=\frac{u^{n+1}-u^{n}}{\Delta t}
$$

with time step $\Delta t$ and superscript indicating the current time, $n$ or the following time step $n+1$. This can be substituted into an example PDE $u_{x x}=u_{t}$ as

$$
u_{x x}^{n}=\frac{u^{n+1}-u^{n}}{\Delta t}
$$


which in matrix form using shape functions becomes

$$
\mathbf{N}_{\mathbf{x x}} \mathbf{U}^{n}=\frac{\mathbf{U}^{n+1}-\mathbf{U}^{n}}{\Delta t}
$$

and can then be rearranged as follows

$$
\left[\Delta t \mathbf{N}_{\mathbf{x x}}+\mathbf{I}\right] \mathbf{U}^{n}=\mathbf{U}^{n+1}
$$

which shows this to be a very straightfoward method for time stepping and can be seen to be an explicit method as it does not require matrix decompositions, only matrix multiplications, and thus is less computationally intensive. This method is implemented and used in a later section on Maxwell's equations as the larger number of variables, three vectors for each of two fields for every point, does not readily allow for matrix inversions.

A second method for time stepping which is more complex than the forward Euler method is the trapezoidal method,

$$
u^{n+1}=u^{n}+\frac{\Delta t}{2}\left(u_{t}^{n+1}+u_{t}^{n}\right)
$$

This method has its region of stability covering the entire left half plane, making it very stable for arbitrarily large time steps, and not at all infringing on the right half plane, allowing it to be used with hyperbolic PDEs. Rearranging this equation gives

$$
\frac{1}{\Delta t} u^{n}+\frac{1}{2} u_{t}^{n}=\frac{1}{\Delta t} u^{n+1}-\frac{1}{2} u_{t}^{n+1}
$$


substituting into matrix form and having $\mathbf{U}_{\mathbf{x x}}=\mathbf{U}_{\mathbf{t}}$ gives

$$
\frac{1}{\Delta t} \mathbf{U}^{n}+\frac{1}{2} \mathbf{U}_{\mathbf{x x}}{ }^{n}=\frac{1}{\Delta t} \mathbf{U}^{n+1}-\frac{1}{2} \mathbf{U}_{\mathbf{x x}}{ }^{n+1},
$$

and lastly rearranging to give a final matrix equation:

$$
\left[\frac{1}{\Delta t} \mathbf{I}+\frac{1}{2} \mathbf{N}_{\mathbf{x x}}\right] \mathbf{U}^{n}=\left[\frac{1}{\Delta t} \mathbf{I}-\frac{1}{2} \mathbf{N}_{\mathbf{x x}}\right] \mathbf{U}^{n+1}
$$

As can be seen, this formulation will require a matrix decomposition and is thus an implicit method.

Another complexity in solving these types of equations is that they are second order in time. However, as shown above, all of the time stepping methods described are for problems that are first order in time. A modification to the equations can be performed to solve this problem. This involves splitting the time derivative into two first order terms, which are solved simultaneously, as seen below

$$
\begin{aligned}
\nabla^{2} u & =C \frac{\partial w}{\partial t} \\
w & =\frac{\partial u}{\partial t}
\end{aligned}
$$

or in matrix form

$$
\begin{aligned}
\mathbf{N}_{\mathbf{x x}} \mathbf{U} & =C \frac{\partial \mathbf{W}}{\partial t} \\
\mathbf{W} & =\frac{\partial \mathbf{U}}{\partial t} .
\end{aligned}
$$

Grouping the entire left hand side of the previous equation into one large matrix, labelled $\tilde{\mathbf{N}}$, and creating a new vector $\tilde{\mathbf{U}}=\left[\mathbf{U}^{\mathbf{T}} \mathbf{W}^{\mathbf{T}}\right]^{T}$, with superscript $\mathrm{T}$ being the 
transpose, we can have

$$
\tilde{\mathbf{N}} \tilde{\mathbf{U}}=\left[\begin{array}{cc}
0 & \frac{1}{C} \mathbf{N}_{\mathbf{x x}} \\
\mathbf{I} & 0
\end{array}\right]\left[\begin{array}{l}
\mathbf{U} \\
\mathbf{W}
\end{array}\right]=\frac{\partial \tilde{\mathbf{U}}}{\partial t}
$$

which becomes a PDE with a first order time derivative. The trapezoid rule can then become

$$
\left[\frac{1}{\Delta t} \mathbf{I}+\frac{1}{2} \tilde{\mathbf{N}}\right][\tilde{\mathbf{U}}]^{n}=\left[\frac{1}{\Delta t} \mathbf{I}-\frac{1}{2} \tilde{\mathbf{N}}\right][\tilde{\mathbf{U}}]^{n+1}
$$

Using the above formulation the FCM can be used to solve the scaler wave equation with the appropriate use of the shape matrix to form the matrix operator representing the second order spatial derivative. The solution of the equation for multiple material regions requires the use of the shape function derived first order spatial derivatives to specify the interface equations and ensure continuity in the energy flow across the interface. This scalar wave equation has been successfully implemented for one and two dimensional materially inhomogeneous problems and the engine created has been used to solve the following equations.

\subsection{Schrodinger's equation}

A wave-like equation of considerable interest is Schrodinger's equation. Schrodinger's equation is a coupled complex wave equation which describes the quantum state of an object and how it evolves through time. The full time dependent equation [1],

$$
-\frac{\hbar^{2}}{2 m} \frac{\partial^{2}}{\partial x^{2}} \Psi(x, t)+V(x) \Psi(x, t)=i \hbar \frac{\partial}{\partial t} \Psi(x, t)
$$


describes the physical object in question having mass $m$, moving in a region with a varying potential field, $V(x)$. The wave function, $\Psi$, when squared, represents the probability of the particle in question being in any particular region. Thus, being a probability, there are normality conditions which require the integral over the square of the wave function to be unity.

The full time dependent Schrodinger equation in one dimension, seen in Eq. (114), can be represented in matrix form by

$$
\frac{\hbar^{2}}{2 m}\left[\begin{array}{cc}
\mathbf{N}_{\mathbf{x x}}+\mathbf{V} & 0 \\
0 & \mathbf{N}_{\mathbf{x x}}+\mathbf{V}
\end{array}\right]\left[\begin{array}{l}
\boldsymbol{\Psi}_{\mathbf{r e}} \\
\boldsymbol{\Psi}_{\mathbf{i m}}
\end{array}\right]=\left[\begin{array}{cc}
0 & -\hbar \\
\hbar & 0
\end{array}\right] \frac{\partial}{\partial t}\left[\begin{array}{l}
\boldsymbol{\Psi}_{\mathbf{r e}} \\
\boldsymbol{\Psi}_{\mathbf{i m}}
\end{array}\right]
$$

and is used in this thesis to solve for both the real and imaginary components.

The solutions to the time dependent Schrodinger equation has a form as follows,

$$
\Psi(x, t)=\psi(x) e^{i(p x+E t)}
$$

which has a wave packet, $\psi(x)$, traveling with energy, $E$, and momentum, $p$. As an initial condition, a wavefunction can be created and modulated with an appropriate phase between the real and imaginary components to give the wavefunction a desired momentum.

In a stationary state the energy of the Schrodinger equation is constant and can be solved as an eigenvalue equation, which results in both a probability space and energy level for each eigenvector -value pair. This time independent equation is [6]

$$
-\frac{\hbar^{2}}{2 m} \frac{d^{2} \psi}{d x^{2}}+V(x) \psi(x)=E \psi(x)
$$


and having a matrix form

$$
\left[-\frac{\hbar^{2}}{2 m} \mathbf{N}_{\mathbf{x x}}+\mathrm{V}\right] \psi_{\mathbf{n}}=E_{n} \psi_{n}
$$

with energy levels, $E_{n}$, and the corresponding eigenvectors, $\Psi_{n}$, with $n=1,2, \ldots$.

We have begun by solving the time independent Schrodinger equation for simple structures, a particle in a homogeneous box, in one, two and three dimensions. This problem has previously been studied in [15]. The problem will then be extended to particles in a box with an applied electric field, varying material properties, and time dependent solutions.

\subsubsection{Particle in a box}

Initial tests of solving the time independent Schrodinger's Equation are on the simplest structure, the particle in an infinite quantum well. The problem is described as

$$
-\frac{\hbar^{2}}{2 m} \nabla^{2} \psi=E \psi
$$

with no potential in the well and infinite potential outside the well. The well in our tests has width $5 \AA$ centred about the origin. The potential has $V(x)=0, a<$ $x<a$ and $V(a)=V(-a)=$ inf, $a=2.5 \AA$. These Dirichlet boundary conditions restrict $\psi(x)=0$ at the boundaries due to the infinite potential, and the solution domain is discretized into 100 points. This problem is easily solvable and gives rise to eigenfunctions with given eigenvalues which are physically treated as probability distributions with given quantized energy levels. The particle in a box of width $a$ has 
solutions [1]

$$
\psi_{n}(x)=\sqrt{\frac{2}{a}} \sin \frac{n \pi x}{a}, \quad E_{n}=\frac{\hbar^{2} \pi^{2} n^{2}}{2 m a^{2}}
$$

This simple test has been solved using the FCM and Fig. 57 shows the first 4 eigenvectors and Table 5 compares the determined eigenenergies with the known values. The results show a very good agreement between the known solution and the FCM.

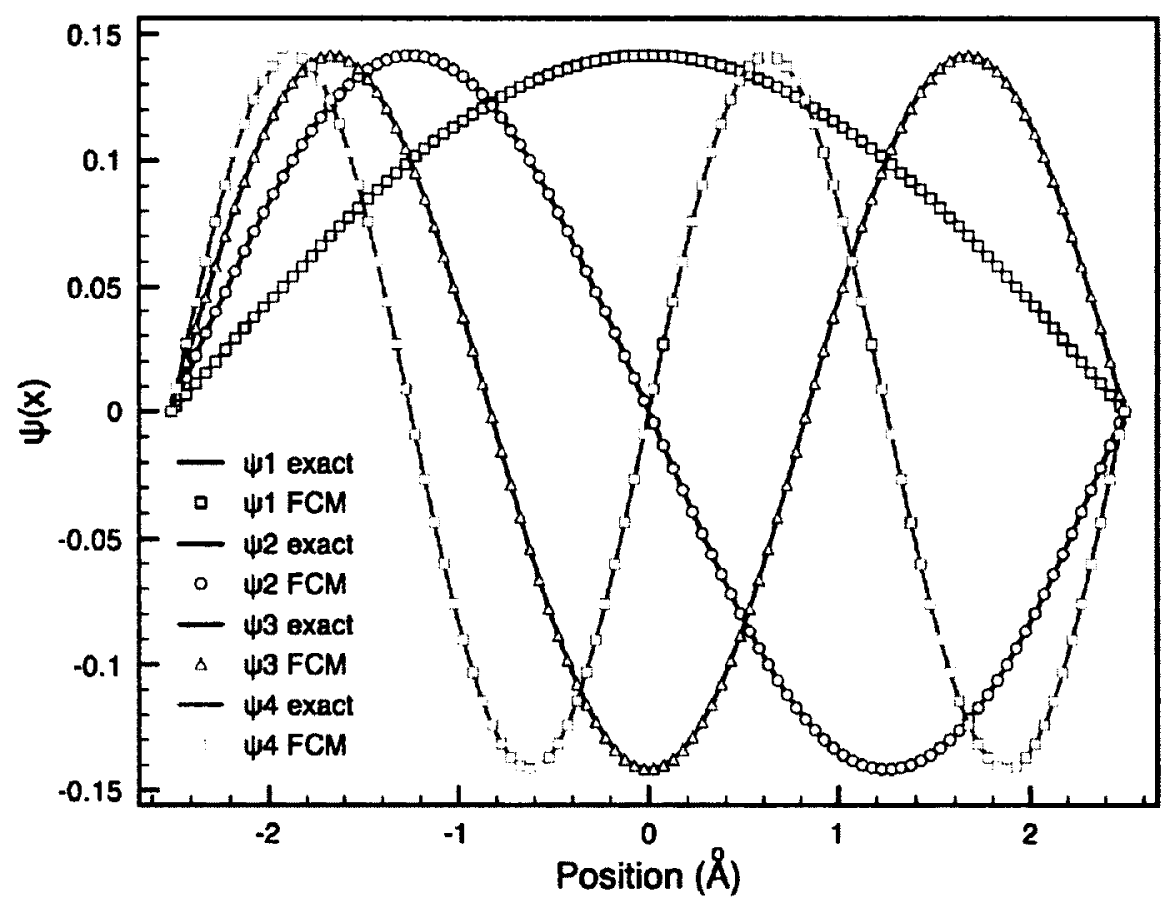

Figure 57: First four eigenvectors from the 1D particle in an infinite box of $0.5 \mathrm{~nm}$ width, 100 points, node values shown as the points and theoretical known solution as the solid line.

With two or three dimensions the process is similar, however the solutions require two or three subscripts to identify the mode in question, typically $n, m, l$. Similar 2D and $3 \mathrm{D}$ tests were performed with point distributions $(100 \times 50)$ and $(20 \times 30 \times 40)$ 
Table 5: Energies for the first four energy levels of the 1D particle in a box compared with known solutions and the corresponding percent error.

\begin{tabular}{c|c|c|c}
$\mathrm{n}$ & $\mathrm{E}_{n(F C M)}(\mathrm{eV})$ & $\mathrm{E}_{n(k n o w n)}(\mathrm{eV})$ & Percent Error $(\%)$ \\
\hline 1 & 1.504 & 1.504 & -0.008 \\
2 & 6.015 & 6.016 & -0.032 \\
3 & 13.527 & 13.537 & -0.077 \\
4 & 24.035 & 24.066 & -0.128 \\
\hline
\end{tabular}

Table 6: Energies for the first nine energy levels of the 2D particle in a box compared with known solutions and the corresponding percent error.

\begin{tabular}{c|c|c|c|c}
$\mathrm{n}$ & $\mathrm{m}$ & $\mathrm{E}_{n m(F C M)}(\mathrm{eV})$ & $\mathrm{E}_{n m(k n o w n)}(\mathrm{eV})$ & Percent Error (\%) \\
\hline 1 & 1 & 1.880 & 1.880 & -0.023 \\
2 & 1 & 3.007 & 3.008 & -0.030 \\
3 & 1 & 4.885 & 4.888 & -0.060 \\
1 & 2 & 6.385 & 6.393 & -0.116 \\
2 & 2 & 7.512 & 7.521 & -0.109 \\
4 & 1 & 7.513 & 7.521 & -0.106 \\
3 & 2 & 9.391 & 9.401 & -0.108 \\
5 & 1 & 10.886 & 10.905 & -0.178 \\
4 & 2 & 12.017 & 12.033 & -0.130 \\
\hline
\end{tabular}

respectively. Their eigen energies are shown in Tables 6 and 7, as well a solution for the eigenvector with $n=4, m=2$ in the $2 \mathrm{D}$ simulation is plotted in Fig. 58. A higher percent error is expected for the $3 \mathrm{D}$ case as a smaller number of points are used in each dimension.

\subsubsection{Particle in a finite well}

A more realistic problem involves a non-infinite well, in this case a simple change in potential $V(x)$, which can be physically created using a material heterostructure. 


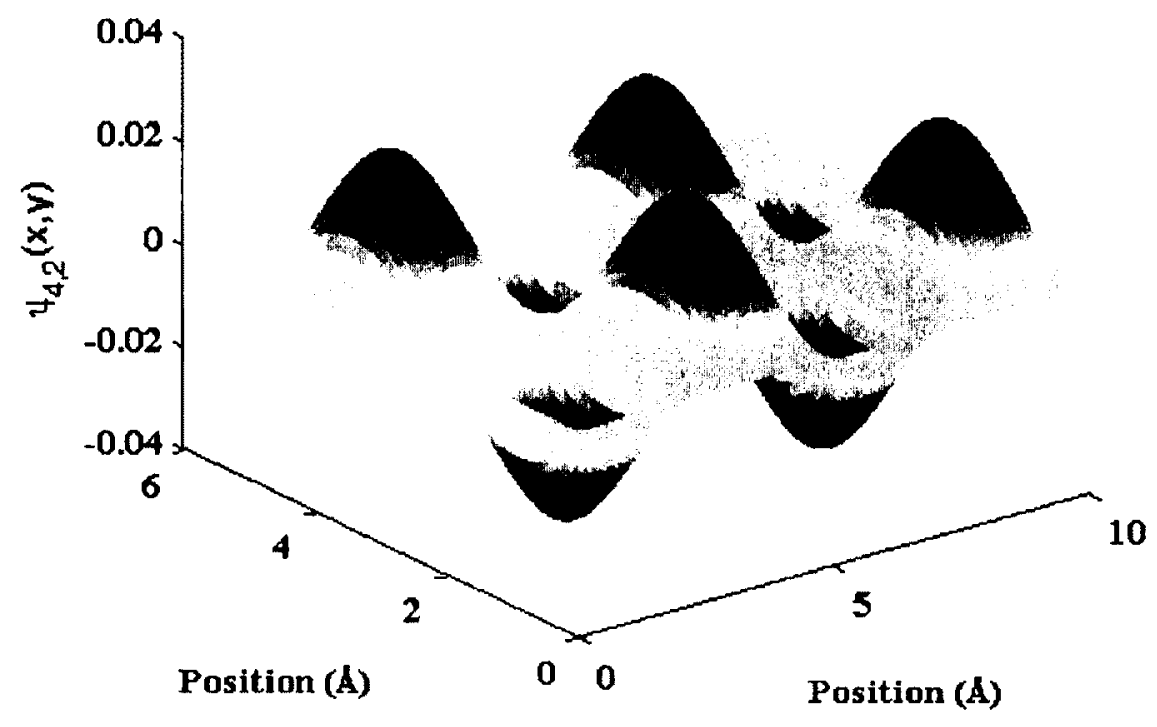

Figure 58: Solution to the 2D particle in a box simulation showing the eigenvector $\psi(4,2)$ for an infinite box of dimensions $1 \mathrm{~nm} \times 0.5 \mathrm{~nm}$.

Table 7: Energies for the first nine energy levels of the 3D particle in a box compared with known solutions and the corresponding percent error.

\begin{tabular}{c|c|c|c|c|c}
$\mathrm{n}$ & $\mathrm{m}$ & $\mathrm{l}$ & $\mathrm{E}_{n m l(F C M)}(\mathrm{eV})$ & $\mathrm{E}_{n m l(k n o w n)}(\mathrm{eV})$ & Percent Error (\%) \\
\hline 1 & 1 & 1 & 15.906 & 15.929 & -0.145 \\
1 & 1 & 2 & 22.937 & 22.980 & -0.185 \\
1 & 2 & 1 & 28.384 & 28.463 & -0.278 \\
1 & 1 & 3 & 34.611 & 34.731 & -0.345 \\
1 & 2 & 2 & 35.413 & 35.514 & -0.284 \\
2 & 1 & 1 & 43.856 & 44.131 & -0.625 \\
1 & 2 & 3 & 47.083 & 47.265 & -0.384 \\
1 & 3 & 1 & 49.044 & 49.354 & -0.627 \\
2 & 1 & 2 & 50.861 & 51.182 & -0.627 \\
\hline
\end{tabular}


This quantum well can be created by sandwiching a layer of one material (eg. GaAs) between layers of a second material (eg. AlGaAs). The change in conduction levels creates a potential well for electrons in the conduction band.

Electrons in these materials also have an effective mass, which describes the motion of the electron under the influence of a potential. The differing material properties give rise to varying effective masses on either side of the junction of the two materials. Under typical situations with a homogeneous material the derivative of the wave function must be constant to ensure conservation of current giving

$$
\left.\frac{d \psi}{d x}\right|_{x=a}=\left.\frac{d \psi}{d x}\right|_{x=b}
$$

with the derivatives being taken from region $a$ or region $b$. A modification to the interface condition must be made to include the effective mass for heterojunctions, which will ensure a conservation of current across the junction. The matching condition is as follows,

$$
\left.\frac{1}{m_{a}} \frac{d \psi}{d x}\right|_{x=a}=\left.\frac{1}{m_{b}} \frac{d \psi}{d x}\right|_{x=b}
$$

and can be enforced and solved in the usual manner, the s-FCM, with $m_{a}$ and $m_{b}$, the effective mass of the particle in material $a$ and $b[1]$.

An example is shown using a well of $5 \mathrm{~nm}$ width and $1 \mathrm{eV}$ deep with effective mass in the well of $m_{W}=0.067$. A solution is shown for two barrier effective masses and the entire domain uses 450 points. The first three energy levels are compared for barriers of effective mass $m_{B}=0.067$ and $m_{B}=0.15$. Results for this example are shown in Table 8 and their eigenfunctions are shown in Fig. 59 for the center 200 points using the case of the larger barrier mass. 
Table 8: Wave function and energy for the first 3 energy levels of the 1D particle in a finite, $3 \mathrm{eV}$, well with effective mass in the well $m_{W}=0.067$ and barrier effective masses of $m_{B}=0.067$ and $m_{B}=0.15$, compared with known solutions [1], showing the center 200 points of a total 450 points used in the simulation.

\begin{tabular}{c|c|c|c|c}
$\mathrm{n}$ & $\mathrm{E}_{n(F C M)}(\mathrm{eV})$ & $\mathrm{E}_{n(\text { known })}(\mathrm{eV})$ & $\mathrm{E}_{n(F C M)}(\mathrm{eV})$ & $\mathrm{E}_{n(\text { known })}(\mathrm{eV})$ \\
\hline & \multicolumn{2}{|c|}{$m_{B}=0.067$} & \multicolumn{2}{c}{$m_{B}=0.15$} \\
\hline 1 & 0.131 & 0.131 & 0.108 & 0.108 \\
2 & 0.503 & 0.504 & 0.445 & 0.446 \\
3 & 0.980 & 0.981 & 0.968 & 0.969 \\
\hline
\end{tabular}

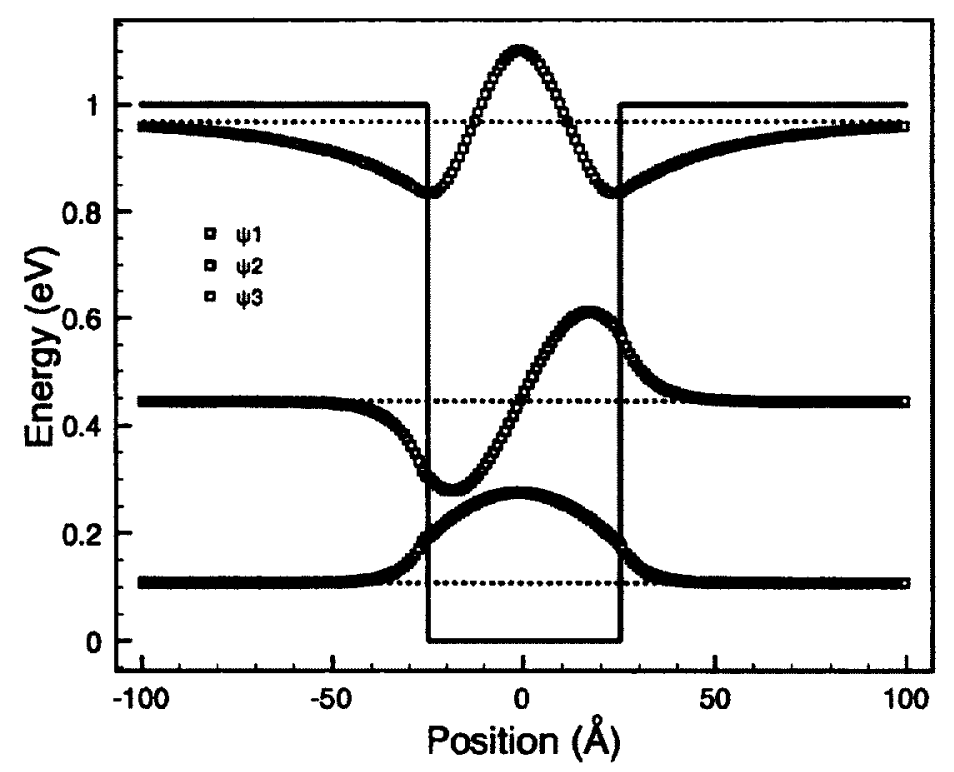

Figure 59: Energies for the first 3 energy levels of the 1D particle in a finite $3 \mathrm{eV}$ well with effective mass in the well $m_{W}=0.067$ and barrier effective mass of $m_{B}=0.15$. 


\subsubsection{Particle in a parabolic well}

The final example presented for this equation is the particle in a parabolic well, with a full time dependent simulation. An initial gaussian distribution with no momentum is assigned to the wave function and its position is $5 \mathrm{~nm}$ from the centre of a parabolic potential well. From analytical consideration it can be shown that the parabolic potential will compensate for the natural wave-function dispersion. The expected result is for the electron wave packet to oscillate between two positions. As expected, the particle's probability function oscillates across the well, broadening in the centre and returning to its original shape at each extremum.

A plot with the potential well and the first 44 time steps, which includes the extremes of the wave's oscillation, is shown in Fig. 60. The total area of the square of the wave function should remain a constant, as it represents the probability function for the particle being in the well. After more than 300 time steps the area of the squared function was still within $1.5 \%$ of the expected $100 \%$ area giving a very low total loss for the model.

\subsection{Maxwell's equations}

The last and most complicated partial differential equations looked at in this chapter are Maxwell's curl equations. These equations describe the evolution of electric and magnetic fields and their generation from electric charges, currents and time varying magnetic and electric field's respectively. The equations used are [52]

$$
\begin{aligned}
& \frac{\partial H}{\partial t}=\frac{1}{\mu_{0}} \nabla \times E \\
& \frac{\partial E}{\partial t}=\frac{1}{\varepsilon_{0}} \nabla \times H-\frac{\sigma}{\varepsilon_{0}} E,
\end{aligned}
$$




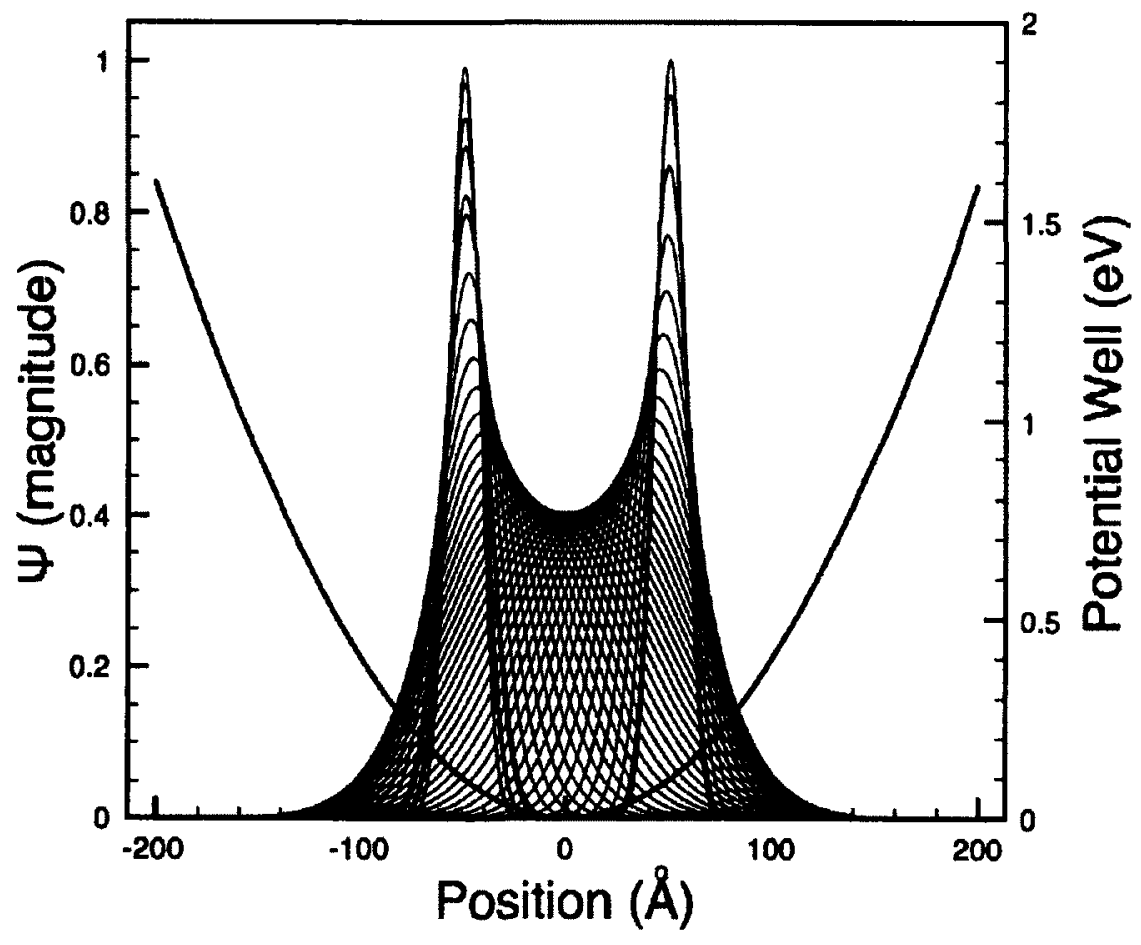

Figure 60: Wave function shown at 44 discrete time steps through both extremes of a one dimensional parabolic well, also shown. 
with $E$ the electric field, $H$ the magnetic field, $\sigma$ the conductance of the material, and $\epsilon_{0}$ the permittivity of free space.

Ignoring for simplicity the conductance of the given material, the initial divergence of both fields can be simulated. These equations are broken up into their corresponding field components and are made ready for the shape function as seen below:

$$
\begin{aligned}
& {\left[\begin{array}{l}
\frac{1}{-\mu_{0}}\left[\frac{\partial E_{z}}{\partial y}-\frac{\partial E_{y}}{\partial z}\right]=\frac{\partial H_{x}}{\partial t} \\
\frac{1}{-\mu_{0}}\left[\frac{\partial E_{x}}{\partial z}-\frac{\partial E_{z}}{\partial x}\right]=\frac{\partial H_{y}}{\partial t} \\
\frac{1}{-\mu_{0}}\left[\frac{\partial E_{y}}{\partial x}-\frac{\partial E_{x}}{\partial y}\right]=\frac{\partial H_{z}}{\partial t}
\end{array}\right],} \\
& {\left[\begin{array}{l}
\frac{1}{\varepsilon_{0}}\left[\frac{\partial H_{y}}{\partial x}-\frac{\partial H_{x}}{\partial y}\right]=\frac{\partial E_{z}}{\partial t} \\
\frac{1}{\epsilon_{0}}\left[\frac{\partial H_{z}}{\partial y}-\frac{\partial H_{y}}{\partial z}\right]=\frac{\partial E_{x}}{\partial t} \\
\frac{1}{\varepsilon_{0}}\left[\frac{\partial H_{x}}{\partial z}-\frac{\partial H_{z}}{\partial x}\right]=\frac{\partial E_{y}}{\partial t} .
\end{array}\right] .}
\end{aligned}
$$

To clarify, the shape matrix $\mathbf{N}$ has spatial derivatives $\mathbf{N}_{\mathbf{x}}$, however with fields such as the electric field, $E$, has components in the x-direction $E_{x}$.

An example of the matrix layout with the shaping functions is

$$
\left[\begin{array}{ccc}
0 & -\mathbf{N}_{\mathbf{z}} & \mathbf{N}_{\mathbf{y}} \\
\mathbf{N}_{\mathbf{z}} & 0 & -\mathbf{N}_{\mathbf{x}} \\
-\mathbf{N}_{\mathbf{y}} & \mathbf{N}_{\mathbf{x}} & 0
\end{array}\right]\left[\begin{array}{l}
\mathbf{E}_{\mathbf{x}} \\
\mathbf{E}_{\mathbf{y}} \\
\mathbf{E}_{\mathbf{z}}
\end{array}\right]=C\left[\begin{array}{l}
\mathbf{H}_{\mathbf{x}} \\
\mathbf{H}_{\mathbf{y}} \\
\mathbf{H}_{\mathbf{z}}
\end{array}\right]^{\prime}
$$

which is used for determining the solution of the time derivative of the H-field, denoted by the prime. The shape function is of a similar form for the solution of the 
time dependent E-field. As mentioned above, the time stepping method used to solve Maxwell's equations in this thesis is the forward Euler method due to the large matrices created in the process, typically having dimensions six times that of a simple scalar problem.

\subsubsection{Node placement}

The placement of nodes for the FCM is different for Maxwell's equations than previous methods. For this method there are two different types of nodes, one representing an electric field, and the other representing the magnetic field. With both fields being represented at the same point, they must be calculated simultaneously due to causality, which adds to the computational difficulty. An alternate approach is to have two types of points offset from each other for the $E$ and $H$ fields. This allows the points to be calculated separately in a leapfrog type manner reducing the computational cost. The scheme however requires careful attention to time step size to ensure stability, which will follow from an analysis of the forward Euler method.

In the FDTD approach a Yee lattice, [53], to offset the field points, Fig. 61, is typically used which is very rigid and can become more difficult in areas with high variability and odd structure shapes. The placement used in this work for the FCM requires all boundary points to be of the same field type, typically the electric field, and have appropriate boundary conditions placed upon them. Moving inward from the exterior boundary the next set of points must be of the opposite field type, magnetic field for this case. The exact distribution of these boundary elements is flexible with a higher density being used in areas with a larger expected gradient. Remaining interior points can be placed in either a regular or irregular distribution depending on the case or desired granularity, with the two field nodes typically offset with each other. 


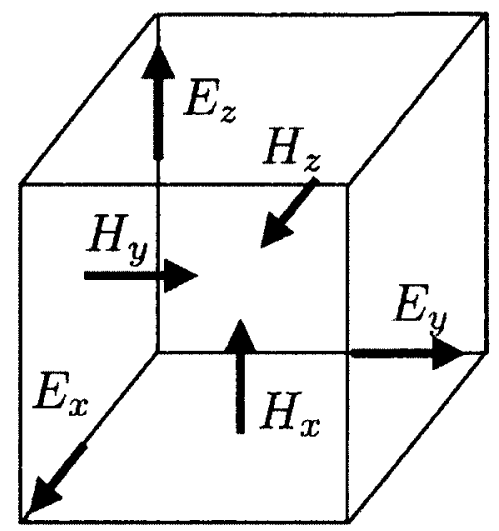

Figure 61: A Yee cell showing the locations of the field vectors being represented in a staggered grid.

\subsubsection{Boundary conditions}

As with all solutions to partial differential equations, proper boundary conditions must be enforced. Our current method uses exclusively $E$ field nodes along the boundaries, with several different boundary conditions having been used thus far in the modelling. The first and simplest boundary condition used is the ideal metallic boundary condition. This forces the E-field parallel to the boundary to equal zero, as there cannot be an electric field inside of a perfectly conducting metal. To enforce this condition one must simply know the normal to the boundary at each node, which can be stored upon geometry layout, and at each E-field iteration the appropriate parallel fields set to zero.

The second boundary condition used is a matching boundary condition, which can be used to simulate a field that continues with no changes in a particular direction. This, for example, can be used on the long narrow sides of a uniformly illuminated guide to 'extend' the dimensions without using extra nodes and processing time. To accomplish this boundary condition the boundary node is typically relocated a half cloud width into the domain for the calculation of that node's shape function. Since the relocation is only done for that particular node's shape function, which is only 
dependent on the $\mathrm{H}$-field nodes, the result is to force the derivative of that particular E-field to zero at the boundary.

\subsubsection{Inhomogeneous solutions}

Due to the different, explicit, nature of solving the EM equations, the previously used methods for heterogeneous materials cannot be used. Instead, a value of the permittivity for a particular node and field direction is calculated. For each node and field direction (eg. $E_{y}$ ) an average permittivity is calculated using nine points on the plane normal to the field direction (eg. $x-z$ plane). An example of this permittivity averaging for each field is shown in Fig. 62. This creates a somewhat 'softer' boundary than the previous methods, however, and is better suited to modelling materials of differing shapes. As well this method does not require a specially created point distribution, however it still allows for the nodes to be placed in a higher density around the material change, or in a pattern following the material boundary. Such a method can also be applied to the conductance and permeability of the materials.

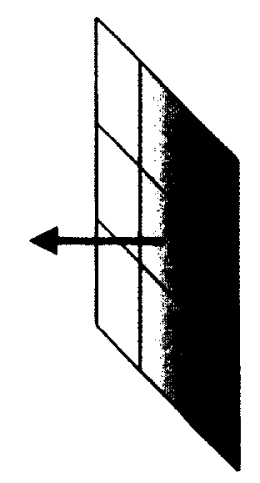

Figure 62: An illustration of a field vector from a node, and the nine points surrounding the plane normal to the vector which are used to calculate the permittivity node and direction. 


\subsubsection{Basic eigenfrequencies}

Due to both the complicated nature of the problems and the computational intensity necessary to solve, only a simple initial problem is presented here; a small test was performed to extract the eigenfrequencies of a simulation space. Nodes were arranged in a one dimensional line, given Dirichlet boundary conditions, and each E-field node excited with a pseudo random value. The field is allowed to oscillate and values at certain points on the line are recorded for several thousand time steps. From these values the Fourier transform can be taken to verify that the correct modes, or eigenfrequencies, are being activated. For a one dimensional EM wave these eigenfrequencies correspond to [54]

$$
f_{m}=\frac{c}{2} \frac{m}{L_{x}}, m=1,2, \ldots
$$

or for two dimensions,

$$
f_{m n}=\frac{c}{2}\left[\frac{m^{2}}{L_{x}}+\frac{n}{L_{y}}\right]^{1 / 2}, m, n=1,2, \ldots
$$

with $L_{x}, L_{y}$ the lengths of the domain in $x$ and $y$ respectively, $c$ the speed of light, and $f_{m n}$ the eigenfrequency for the $m n^{\text {th }}$ mode. A full EM simulation showing the eigenfrequencies in a one dimensional simulation with the expected frequencies is shown in Fig. 63. A second comparison using two dimensions is shown in Fig. 64, where the eigenfrequencies are found by (128). Both simulations show very good agreement with theory for the first several eigenfrequencies and provide a good initial test of the method. The difference in noise in the two examples is due to the averaging of the frequencies found from a larger number of spatial points in two dimensions than one dimension. 


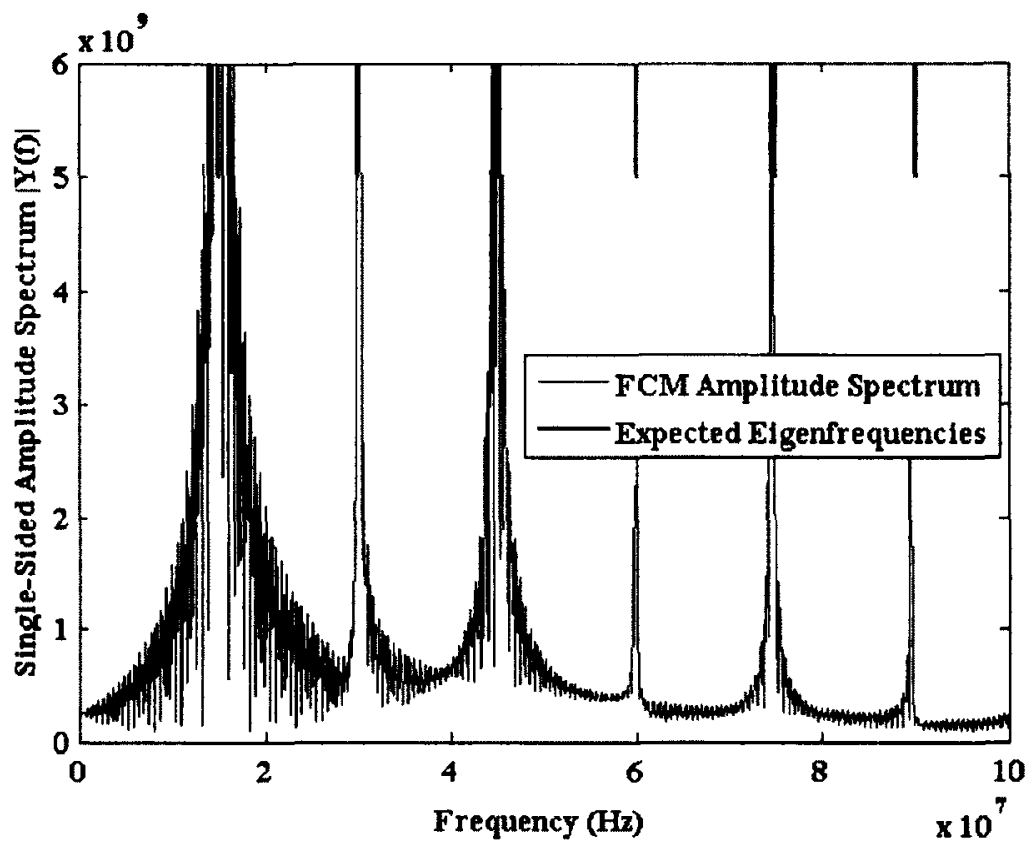

Figure 63: Single sided amplitude spectrum for the one dimensional EM random excitation of a $10 \mathrm{~m}$ line in free space with Dirichlet boundary conditions and the expected oscillation frequencies in black for reference.

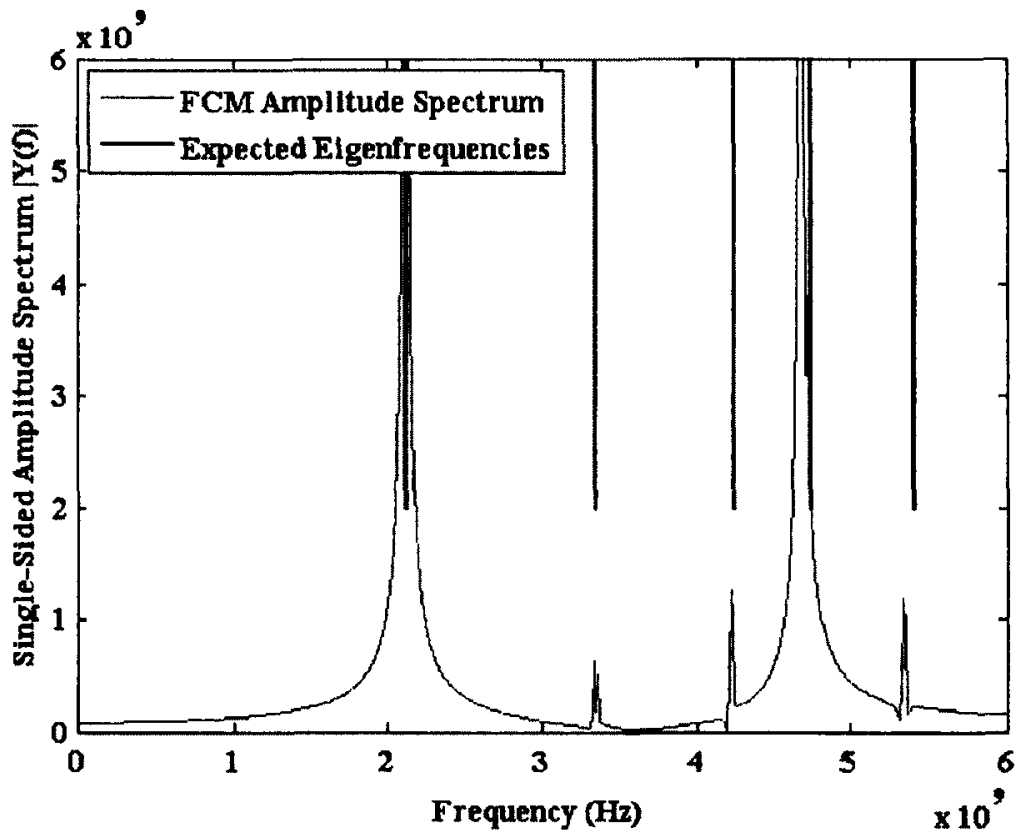

Figure 64: Single sided amplitude spectrum for the two dimensional EM random excitation of a $0.1 \mathrm{~m} \times 0.1 \mathrm{~m}$ square in free space with reflective boundary conditions and the expected oscillation frequencies in black for reference. 


\subsection{Summary}

In this chapter it has been shown that the FCM is able to solve wave type equations of differing forms. Schrodinger's coupled equations are solved for both eigenvalue and transient type problems. Full three dimensional simulations are performed as well as materially inhomogeneous problems which have varying electron effective masses between layers of semiconducting materials.

Maxwell's equations are also attempted for simple initial type problems. It is found that Maxwell's equations are not necessarily best suited to the FCM due to the nature of the fields and the necessity of interweaving of field points. That being said, Maxwell's equations are quite difficult to solve using any type of PDE solver and further research may prove fruitful. In the next section I will show the application of the FCM to a particular case of Maxwell's equation in which these issues are not present. 


\section{Chapter 7}

\section{Mode solving}

After development of the materially inhomogenous form of the FCM, a specific problem of interest was identified - optical waveguide mode solving. The industrial use of optical waveguides with complex geometries is a problem that has attracted both academic and industrial interest.

As optical waveguides become more complex and integrated into optoelectronic circuits there is a need to more accurately model their propagation constants and modes. The guided modes for an optical transmission line describe both the field intensities as well as the effective index of refraction $\left(n_{\text {eff }}\right)$ and group velocity of the signals.

Several commercial mode solvers for this type of problem are available which use the FEM. These solvers are quite advanced and can readily mesh and solve complex microstructured fibres with both regular Neumann/Dirichlet boundaries and absorbing boundary conditions (ABCs). Two of these solvers are FemSIM by RSoft [55], and COMSOL's MultiPhysics [2], which have been used to compare their results against the FCM results. Throughout their use it was found that on occasion the programs could be difficult to use in creating complex shapes which are not predefined, as well as occasionally delivering spurious or incorrect modes. These types of problems were 
also encountered with the use of the FCM mode solving tool. Alternately, other results are compared to known analytic solutions or published results using an FEM or FDM scheme.

\subsection{Mode solving}

This work utilizes coupled $\mathrm{H}$-field full vectorial solutions, and is applied to isotropic non-heterogeneous graded-index or step-index structures which are invariant in the z-direction. The transverse $H_{y}$ and $H_{x}$ fields are determined and the remaining $H$ and $E$ fields can be easily calculated as shown in [56].

Optical propagation along a waveguide is described by Maxwell's equations [57] and is a 2D eigenvalue problem in $(x, y)$ with propagation in the $z$ direction and having a free space propagation constant given by $k_{0}=2 \pi / \lambda_{0}$ with free space wavelength $\lambda_{0}$. The determined eigenvectors $H_{x}$ and $H_{y}$ correspond to the eigenvalue, $\beta$, which is the guided propagation constant which can be used to find the effective refractive index of the mode and material, $n_{\text {eff }}=\beta / k_{0}$.

Two non-coupled equations used for homogeneous isotropic regions are as follows [56]

$$
\begin{aligned}
\frac{\partial^{2} H_{x}}{\partial x^{2}}+\frac{\partial^{2} H_{x}}{\partial y^{2}}+\left(\epsilon_{r} k_{0}^{2}-\beta^{2}\right) H_{x} & =0 \\
\frac{\partial^{2} H_{y}}{\partial x^{2}}+\frac{\partial^{2} H_{y}}{\partial y^{2}}+\left(\epsilon_{r} k_{0}^{2}-\beta^{2}\right) H_{y} & =0
\end{aligned}
$$

with $\epsilon_{r}$ the relative permittivity of the homogeneous material.

When representing the waveguide as coupled regions of constant permittivity (step-index method), the boundary conditions for the $\mathrm{H}$-fields on a junction between two regions of differing refractive index, regions $a$ and $b$, must also be satisfied. These 
conditions also provide a coupling between the $H_{x}$ and $H_{y}$ fields. Ensuring the continuity of the $H_{z}$ field across a junction gives [56]

$$
\left.\frac{\partial H_{x}}{\partial_{x}}\right|_{a}+\left.\frac{\partial H_{y}}{\partial y}\right|_{a}=\left.\frac{\partial H_{x}}{\partial x}\right|_{b}+\left.\frac{\partial H_{y}}{\partial_{y}}\right|_{b},
$$

and as well, ensuring the continuity of the $E_{z}$ field yields [56]

$$
\left.\frac{1}{\varepsilon_{a}} \frac{\partial H_{x}}{\partial_{y}}\right|_{a}-\left.\frac{1}{\varepsilon_{a}} \frac{\partial H_{y}}{\partial_{x}}\right|_{a}=\left.\frac{1}{\varepsilon_{b}} \frac{\partial H_{x}}{\partial_{y}}\right|_{b}-\left.\frac{1}{\varepsilon_{b}} \frac{\partial H_{y}}{\partial_{x}}\right|_{b},
$$

with $\epsilon_{a}$ and $\epsilon_{b}$ being the relative permittivity of regions a and $b$ respectively. The enforcement of these interface conditions will be explained below.

\subsubsection{Eigenmodes and eigenvalues}

To solve for the eigenmodes and eigenvalues one must enforce the above conditions, ensuring that each matrix line includes the eigen-vector and -value on the right hand side of the equation. First the 2D homogeneous equation is rearranged to isolate for the eigenvalues and vectors,

$$
\begin{aligned}
\frac{\partial^{2} H_{x}}{\partial x^{2}}+\frac{\partial^{2} H_{x}}{\partial y^{2}}+\epsilon_{r} k_{0}^{2} H_{x} & =\beta^{2} H_{x} \\
\frac{\partial^{2} H_{y}}{\partial x^{2}}+\frac{\partial^{2} H_{y}}{\partial y^{2}}+\epsilon_{r} k_{0}^{2} H_{y} & =\beta^{2} H_{y}
\end{aligned}
$$

We can apply Eq. (132) and transform it into a single eigenvalue equation of the form,

$$
\mathbf{A X}=\lambda \mathbf{X}
$$

with $\mathbf{A}$ being created using shape functions formed for each node, the local permittivity and its spatial derivatives and the boundary conditions, $\mathbf{X}$ is a vector of $H_{x}$ 
and $H_{y}$ fields at every node and $\lambda=\beta^{2}$ the eigenvalue for a specific mode and can be used to determine the effective index of the mode. Next is shown an example of the implementation of this using the FCM in matrix form,

$$
\left[\begin{array}{cc}
\mathbf{N}_{\mathbf{x x}}+\mathbf{N}_{\mathbf{y y}}+\mathbf{I} \epsilon_{r} k_{0}^{2} & 0 \\
0 & \mathbf{N}_{\mathbf{x x}}+\mathbf{N}_{\mathbf{y y}}+\mathbf{I} \epsilon_{r} k_{0}^{2}
\end{array}\right]\left[\begin{array}{l}
\mathbf{H}_{\mathbf{x}} \\
\mathbf{H}_{\mathbf{y}}
\end{array}\right]=\beta^{2}\left[\begin{array}{l}
\mathbf{H}_{\mathbf{x}} \\
\mathbf{H}_{\mathbf{y}}
\end{array}\right]
$$

with a constant material permittivity, $\epsilon_{r}$, and $\mathbf{I}$ being the identity matrix. When multiple materials are present the appropriate $\epsilon_{r}$ for a particular node must be used in forming these equations.

Typically, mode finding for an equation such as (133) is performed using the appropriate function in a numerical package such as Arpack [58], often called from Matlab [48] or Octave [59]. The function is used to return $n$ eigenvectors whose eigenvalues are closest to the supplied eigenvalue guess. However, a number of issues can arise when using this approach, the returned eigenvectors are not necessarily in the same order with successive calls to the function, in addition if the mode of interest has an eigenvalue near another mode such as the degenerate or quasi-degenerate cases it is possible to have either one of the modes returned.

In the situation where a specific mode is desired and its shape is approximately known, a priori, a method of iteration can be used to find exactly the mode in question. One such method is Rayleigh Quotient Iteration, RQI, as documented in [60]. For this method an eigenvector guess must be supplied along with an approximate eigenvalue. In most cases a rough guess is known and can be interpolated to all of the nodal values in the domain. The RQI method for converging on an eigenvector solution 
from an approximate solution is seen as follows:

Pick a starting vector $\mathbf{X}^{(0)}$ with $\left\|\mathbf{X}^{(0)}\right\|=1$

Pick a starting eigenvalue $\lambda^{(0)}$

For $k=1,2, \ldots$

$$
\begin{aligned}
& \text { Solve }\left(\mathbf{A}-\lambda^{(k-1)} \mathbf{I}\right) w=\mathbf{X}^{(k-1)} \text { for } w \\
& \text { Let } \mathbf{X}^{(k)}=w /\|w\| \\
& \text { Let } \lambda^{(k)}=r\left(\mathbf{X}^{(k)}\right)=\left(\mathbf{X}^{(k)}\right)^{T} \mathbf{A}\left(\mathbf{X}^{(k)}\right)
\end{aligned}
$$

This iteration begins with a starting eigenvector guess, $\mathbf{X}^{(0)}$, shape matrix, $\mathbf{A}$, and eigenvalue guess of $\lambda^{(0)}$ as in Eq. (133). The method then iterates on these values until the solution has converged. The convergence criteria that we use is $\left\|\lambda \mathbf{X}^{(k)}-\mathbf{A} \mathbf{X}^{(k)}\right\|<10^{-9}$.

As will be shown below, the use of RQI will be very useful in improving the robustness and efficiency of the mode solving for cases with complex geometry and boundary conditions.

Interface conditions, which couple the $x$ and $y$ fields, must also be satisfied for the nodes which lie along a material heterojunction. The matrix lines for these nodes must also include the eigenvalues and vectors which are to be solved in this system of equations. Two approaches to interfaces have been implemented and are detailed in the following sections.

\subsubsection{Step index method}

We initially use the step-index method to solve the materially inhomogeneous eigenvalue equations. This stitched method calculates a separate homogeneous cloud for 
both sides of a material junction (clouds "a" and "b"). Each cloud approximates the region on either side of the heterojunction and each is given a weighting factor, which sum to unity, to ensure that the addition of both eigenvalue equations sum to a single eigenvalue. There are also two heterojunction conditions, (130) and (131), which must be enforced. The first condition, Eq. (130) is included in the $H_{x}$ line of the matrix and can be summed along with the weighted eigenvalue equations as,

$$
\begin{aligned}
& M_{a x}\left(\nabla_{a}^{2} H_{x}+k_{0}^{2} \epsilon_{a} H_{x}\right)+M_{b x}\left(\nabla_{b}^{2} H_{x}+k_{0}^{2} \epsilon_{b} H_{x}\right)+ \\
& \left(\left.\frac{\partial H_{x}}{\partial x}\right|_{a}+\left.\frac{\partial H_{y}}{\partial y}\right|_{a}\right)-\left(\left.\frac{\partial H_{x}}{\partial_{x}}\right|_{b}+\left.\frac{\partial H_{y}}{\partial_{y}}\right|_{b}\right)=\beta^{2} H_{x}
\end{aligned}
$$

and the second, Eq. (131) is included in the $H_{y}$ line of the matrix as

$$
\begin{aligned}
& M_{a y}\left(\nabla_{a}^{2} H_{y}+k_{0}^{2} \epsilon_{a} H_{y}\right)+M_{b y}\left(\nabla_{b}^{2} H_{y}+k_{0}^{2} \epsilon_{b} H_{y}\right)+ \\
& \left(\left.\frac{1}{\varepsilon_{a}} \frac{\partial H_{x}}{\partial_{y}}\right|_{a}-\left.\frac{1}{\varepsilon_{a}} \frac{\partial H_{y}}{\partial_{x}}\right|_{a}\right)-\left(\left.\frac{1}{\varepsilon_{b}} \frac{\partial H_{x}}{\partial_{y}}\right|_{b}-\left.\frac{1}{\varepsilon_{b}} \frac{\partial H_{y}}{\partial_{x}}\right|_{b}\right)=\beta^{2} H_{y} .
\end{aligned}
$$

The cloud weighting factor $M_{n m}$ is constructed so that the interface conditions (130) and (131) are satisfied and also that $M_{a x}+M_{b x}=1.0$ and $M_{a y}+M_{b y}=1.0$,

$$
\begin{aligned}
& M_{n x}=\left[\left(\frac{\epsilon_{n}}{\epsilon_{a}+\epsilon_{b}}\right) \cos ^{2} \phi+\left(\frac{1}{2}\right) \sin ^{2} \phi\right] \\
& M_{n y}=\left[\left(\frac{\epsilon_{n}}{\epsilon_{a}+\epsilon_{b}}\right) \sin ^{2} \phi+\left(\frac{1}{2}\right) \cos ^{2} \phi\right]
\end{aligned}
$$

with $\phi$ being the angle normal to the plane of the interface measured counter-clockwise from the positive $\mathrm{x}$-axis. The weighting factor, seen in (138), comes about due to the inverse weighting of the fields perpendicular to the interface normal seen in (131), and ensures that the values on the right hand side of the equation sum to unity giving 
exclusively $\beta^{2} H$ on the right hand side.

\subsubsection{Graded index method}

While performing a literature review on mode solving and computational mode solving routines, it was noted that there are occasions which have a spatially varying index of refraction, or more generally, spatially varying material properties. The method used for solving material inhomogeneities, the stitched method, is unable to include such models as it uses hard boundaries and material discontinuities. A second method for material inhomogeneous models was then necessary to be able to include such spatially varying properties.

For the second method, which we call the graded method or g-FCM, the entire domain is treated as one large homogeneous region with each finite cloud being assigned its own material properties and material spatial derivatives. It is also necessary to adapt any PDE to include the derivatives of the material properties. Such a method does not need to enforce any interface conditions, and can model slowly varying properties as well as sharp transitions given enough points to approximate the material change.

An example of what this may look like with a series of points, a slowly varying material property and the given cloud is shown in Fig. 65(b), included as well is the example stitched cloud, Fig. 65(a).

To account for this, the previous mode solving eigenvalue equation, (132), needs to be appropriately modified to include the spatially varying index of refraction or 


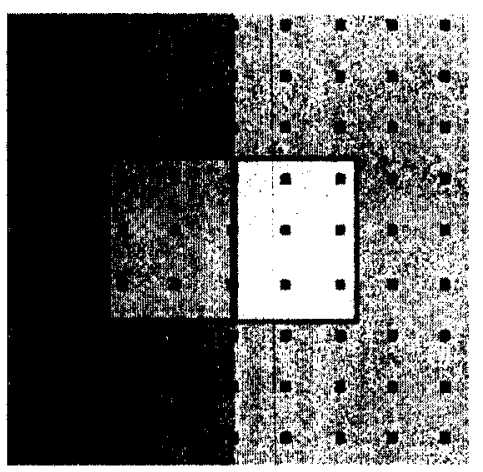

(a)

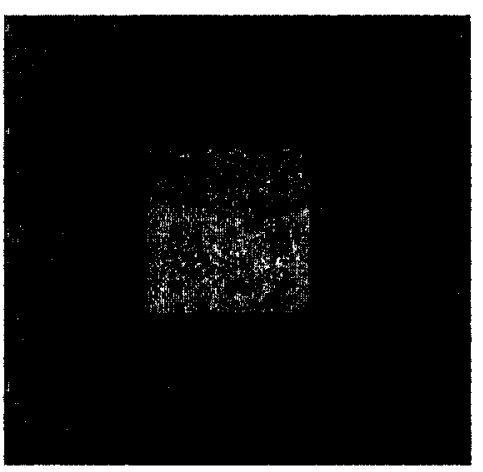

(b)

Figure 65: (a) An example cloud from the stitched method with nodes separated into two regions, showing interface nodes and two 'half' clouds, each extending into only one region. (b) An example cloud using the graded method with all nodes in one continuous region and a single cloud over a varying material property.

permittivity [61],

$$
\begin{aligned}
& \frac{\partial^{2} H_{x}}{\partial x^{2}}+\frac{\partial^{2} H_{x}}{\partial y^{2}}-\frac{1}{\epsilon} \frac{\partial \epsilon}{\partial y} \frac{\partial H_{x}}{\partial y}+\frac{1}{\epsilon} \frac{\partial \epsilon}{\partial y} \frac{\partial H_{y}}{\partial x}+\epsilon_{r} k_{0}^{2} H_{x}=\beta^{2} H_{x} \\
& \frac{\partial^{2} H_{y}}{\partial x^{2}}+\frac{\partial^{2} H_{y}}{\partial y^{2}}-\frac{1}{\epsilon} \frac{\partial \epsilon}{\partial x} \frac{\partial H_{y}}{\partial x}+\frac{1}{\epsilon} \frac{\partial \epsilon}{\partial x} \frac{\partial H_{x}}{\partial y}+\epsilon_{r} k_{0}^{2} H_{y}=\beta^{2} H_{y}
\end{aligned}
$$

which can be transformed into an eigenvalue equation similar to Eq. (134) by direct application of $\mathbf{N}$ and its derivatives as

$$
\begin{aligned}
& {\left[\begin{array}{cc}
\mathbf{N}_{\mathbf{x x}}+\mathbf{N}_{\mathbf{y y}}-\frac{1}{\epsilon} \frac{\partial \epsilon}{\partial y} \mathbf{N}_{\mathbf{y}}+\mathbf{I} \epsilon_{r} k_{0}^{2} & \frac{1}{\epsilon} \frac{\partial \epsilon}{\partial y} \mathbf{N}_{\mathbf{x}} \\
\frac{1}{\epsilon} \frac{\partial \epsilon}{\partial x} \mathbf{N}_{\mathbf{y}} & \mathbf{N}_{\mathbf{x x}}+\mathbf{N}_{\mathbf{y y}}-\frac{1}{\epsilon} \frac{\partial \epsilon}{\partial x} \mathbf{N}_{\mathbf{x}}+\mathbf{I} \epsilon_{r} k_{0}^{2}
\end{array}\right]\left[\begin{array}{l}
\mathbf{H}_{\mathbf{x}} \\
\mathbf{H}_{\mathbf{y}}
\end{array}\right]} \\
& =\beta^{2}\left[\begin{array}{l}
\mathbf{H}_{\mathbf{x}} \\
\mathbf{H}_{\mathbf{y}}
\end{array}\right] \text {. }
\end{aligned}
$$


This method can be used to include materials which have a slowly varying physical parameter, but can also be used to create sharp interfaces.

\subsubsection{Remaining fields}

The remaining field vectors which are not solved in the above manner can be determined as shown in [56]. With the planar $H$ fields determined, the longitudinal components of the field vectors $H_{z}$ and $E_{z}$ must be continuous at the interfaces. From this, and knowing $\nabla \cdot \vec{H}=0$, one can calculate $H_{z}$ as:

$$
H_{z}=\frac{1}{j \beta}\left(\frac{\partial H_{x}}{\partial x}+\frac{\partial H_{y}}{\partial y}\right)
$$

The component of electric field in the $z$ direction, $E_{z}$, can be calculated using the curl of the magnetic field $\nabla \times \vec{H}=j \omega \varepsilon_{0} \varepsilon \vec{E}$ as:

$$
E_{z}=\frac{1}{j \varepsilon k_{0}} \sqrt{\frac{\mu_{0}}{\varepsilon_{0}}}\left(\frac{\partial H_{y}}{\partial x}-\frac{\partial H_{x}}{\partial y}\right)
$$

Lastly the $E_{x}$ and $E_{y}$ fields can be found using:

$$
\begin{aligned}
& E_{x}=\frac{\beta}{\varepsilon k_{0}} \sqrt{\frac{\mu_{0}}{\varepsilon_{0}}} H_{y}-\frac{1}{\varepsilon k_{0} \beta} \sqrt{\frac{\mu_{0}}{\varepsilon_{0}}}\left(\frac{\partial^{2} H_{x}}{\partial x \partial y}+\frac{\partial^{2} H_{y}}{\partial y^{2}}\right) \\
& E_{y}=\frac{\beta}{\varepsilon k_{0}} \sqrt{\frac{\mu_{0}}{\varepsilon_{0}}} H_{x}+\frac{1}{\varepsilon k_{0} \beta} \sqrt{\frac{\mu_{0}}{\varepsilon_{0}}}\left(\frac{\partial^{2} H_{x}}{\partial x^{2}}+\frac{\partial^{2} H_{y}}{\partial x \partial y}\right) .
\end{aligned}
$$

These remaining fields can be easily calculated using the shape functions which would have been calculated to solve for the $H_{x}$ and $H_{y}$ fields, along with matrix multiplications, requiring no new derivative calculations or decompositions. 


\subsubsection{Symmetry}

In cases where there is a physical symmetry in the waveguide, the domain of the simulation can be reduced to save on computational resources with the use of symmetry boundary conditions. For instance, fibers which have either a symmetry in the $x$ or $y$ planes (or both) can be divided in half or a quarter, thus significantly reducing the computational cost. The plane of symmetry then needs to enforce a different set of boundary conditions. These conditions are the Perfect Electric Conductor (PEC),

$$
H_{\perp}=0
$$

and the Perfect Magnetic Conductor (PMC),

$$
H_{\|}=0
$$

Solution accuracy for a variety of fibers will be compared for a full solution, half symmetry and quarter symmetry. The time required to create the domain, matrices and solve the eigenvalue equation, as well as the domain size are also compared for the three symmetry situations. In this thesis the use of symmetry was investigated by the simple creation of a reduced geometric structure and then the imposition of appropriate boundary conditions, however, other more mathematical approaches would also be possible $[62,63]$.

\subsection{Convergence and meshing}

The first test using the FCM and mode solving is performed using a simple step-index fiber with a solid core of slightly higher refractive index than its outer cladding. This is a standard fiber type and the modes and the effective indices of refraction can 


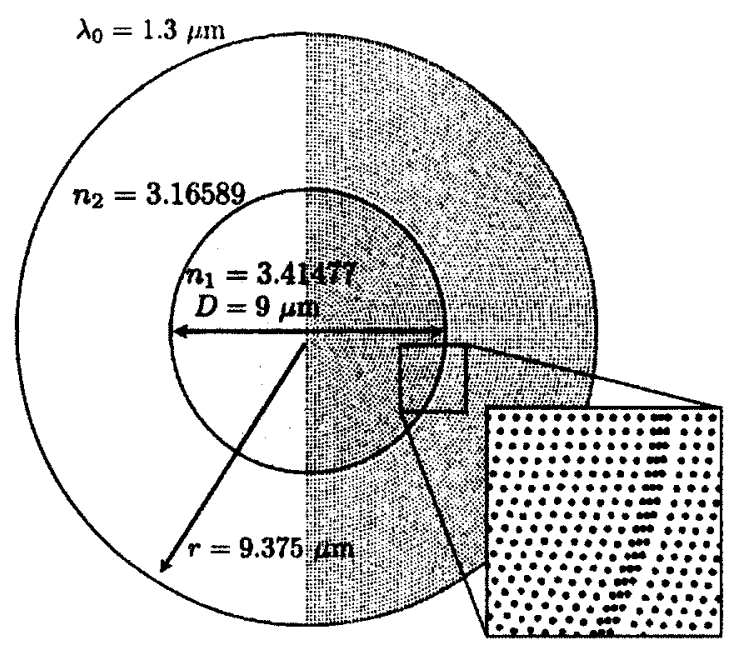

Figure 66: Parameters and dimensions for a solid core step-index fiber used for convergence studies, showing the entire solution domain with an example point layout.

be solved, using the characteristic equation, giving a good test of convergence. The example fiber used is shown in Fig. 66 giving dimensions and physical parameters.

The characteristic equation for this type of fiber is [64]

$$
\left(\frac{J_{m}^{\prime}(\kappa a)}{\kappa J_{m}(\kappa a)}+\frac{K_{m}^{\prime}(\gamma a)}{\gamma K_{m}(\gamma a)}\right)\left(\frac{J_{m}^{\prime}(\kappa a)}{\kappa J_{m}(\kappa a)}+\frac{n_{2}^{2}}{n_{1}^{2}} \frac{K_{m}^{\prime}(\gamma a)}{\gamma K_{m}(\gamma a)}\right)=\left(\frac{m \beta k_{0}\left(n_{1}^{2}-n_{2}^{2}\right)}{a \kappa^{2} \gamma^{2} n_{1}}\right)^{2}
$$

with $k_{0}=2 \pi / \lambda_{0}, J_{m}$ and $K_{m}$, the Bessel function and modified Bessel function of order $m$, with primes indicating differentiation with respect to the argument, $\kappa=$ $n_{1}^{2} k_{0}^{2}-\beta^{2}, a$ the radius of the inner core, $\gamma=\left(\beta^{2}-n_{2}^{2} k_{0}^{2}\right)^{1 / 2}$, and $n_{1}$ and $n_{2}$ the refractive index of the core and cladding respectively. The equation may have several solutions for each integer value of $m$ giving the solutions a notation $\beta_{m n}$ with $m$ and $n$ integers. These mode solutions are designated $H E_{m n}$ and $E H_{m n}$. Modes having $m=0$ correspond to the transverse-electric (TE) and transverse-magnetic (TM) modes of planar waveguides. The equation will not be further discussed and is only included as this full form of the equation can be difficult to locate and having both the equation and reference may be of use to readers. 

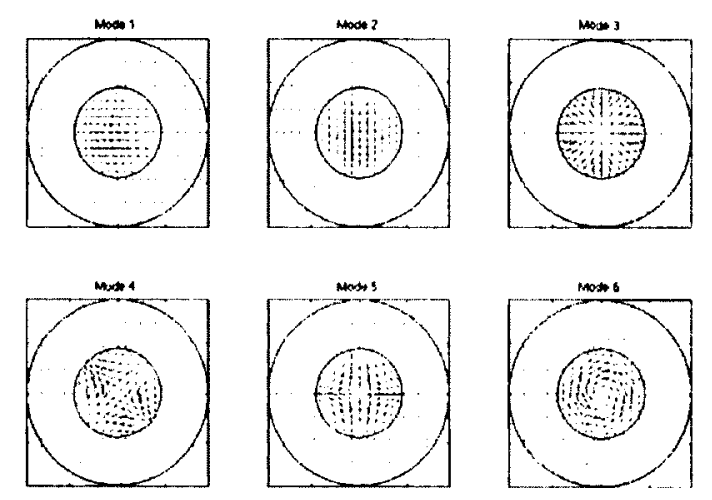

Figure 67: First six modes of the step index fiber.

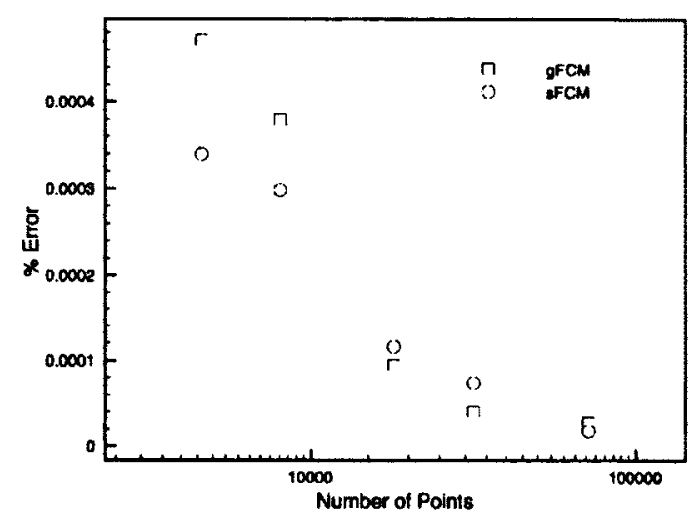

Figure 68: Convergence of the first mode of the step index fibre for both the $\mathrm{g}$-FCM and s-FCM methods plotted against total number of points using a constant density radial distribution.

Having found analytic solutions for the first six modes of the step index fiber, we now compare the results to a variety of point densities using a radial distribution as seen in Fig. 66. The method is shown to converge for all six modes, however plotted only for the fundamental mode, Fig. 68, with the s-FCM giving a smaller error than the g-FCM. The first six modes of this fiber are also shown in Fig. 67.

One of the advantages of a meshless method is the simplicity with which one can vary the point distribution. As mentioned above, the tests have used a constant point density with a radial distribution for this radially symmetric fiber. Knowing 


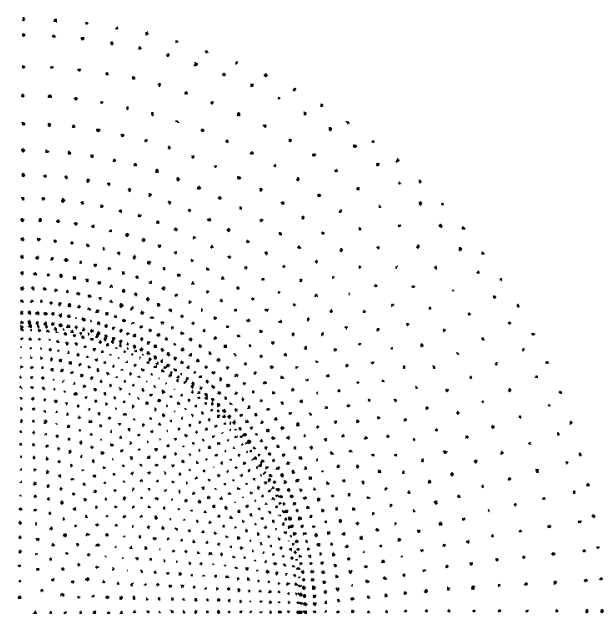

Figure 69: Alternate point distribution for the step index fiber with a point density ratio of 1:4 for the core center to interface (yellow points), and a ratio of 16:1 for the interface to outer cladding (blue points).

that the solution has the highest gradient for the majority of the modes near the corecladding boundary, we can add more nodes in this location while removing nodes from the outer cladding or inner core. An example of this is demonstrated in Fig. 69. Thus for the same total number of nodes we can easily add density in locations of high field gradient and remove nodes with low field gradients. We use a point density ratio of $4: 1$ for the interface to core and a ratio of $16: 1$ for the interface to outer cladding. The error is reduced by approximately half for the same number of points, as shown in Fig. 70.

We have thus shown that the solution is converging to the correct values, and that using simple node distribution changes, the error can be improved. This technique can be easily applied to other structures and will be used later in a more automatic way as adaptive mapping. For the majority of the following tests, node distributions are used which are appropriate for the structures, eg. radial for circular cross sections. Their densities, however, have not been tailored as above. 


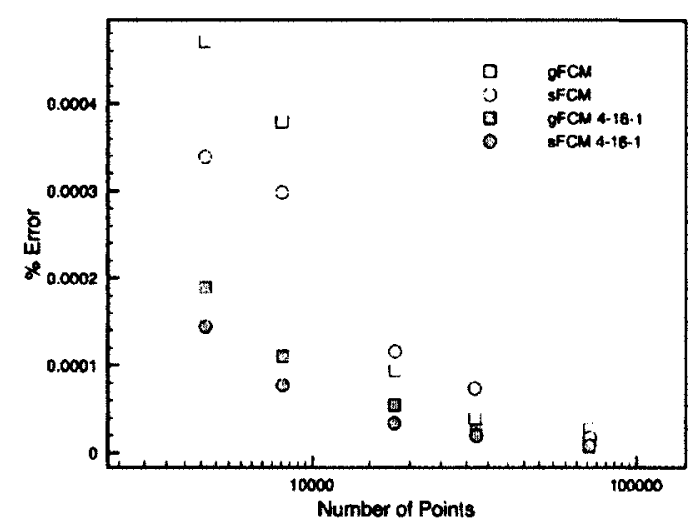

Figure 70: Convergence of the first mode of the step index fibre for both the $g$ FCM and s-FCM methods plotted against total number of points using a point density ratio of $1: 4$ for the core center to interface and a ratio of $16: 1$ for the interface to outer cladding.

\subsection{Guided mode tests}

The following section details initial results of using the FCM to solve for the guided modes of several microstructured optical waveguides. In all cases the figures show the node placements, physical parameters and the entire computational domain of the structure. Dirichlet boundary conditions are used, holding the fields to be zero at the domain edges.

\subsubsection{Optical waveguides}

For an initial investigation of the applicability of the FCM to optical mode solving, two simple waveguides were modeled: 1) a ridge waveguide typical of a Silica on Silicon integrated optical platform and 2) a step-index optical fiber, as shown above in the convergence tests. 


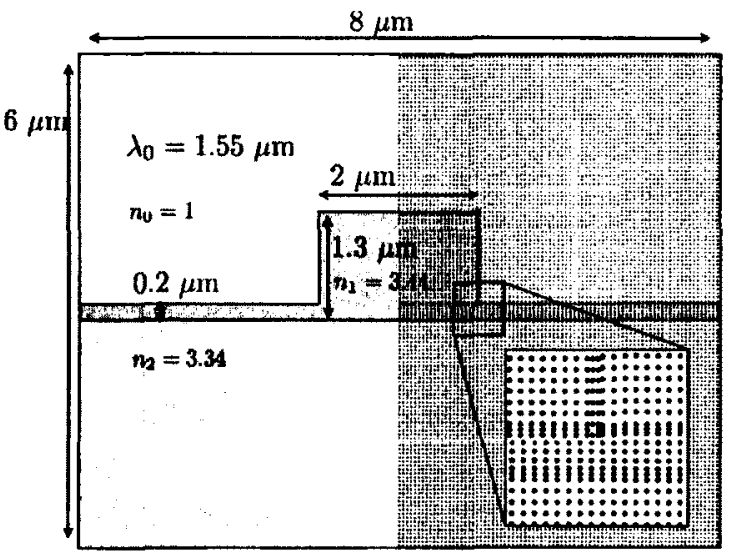

Figure 71: Parameters and dimensions for the ridge waveguide.

\section{Ridge waveguide}

As an initial test, a simple ridge waveguide, with parameters as shown in Fig. 71, was analyzed using the FCM and compared with the solutions from commercial modelling software COMSOL MultiPhysics [2] and Rsoft FemSIM [55]. A similar grid size and computation window were used for all of the solutions, with $d x \approx 0.05 \mu \mathrm{m}$. For the FCM, four material regions were defined. Three layers consisting of a bottom silicon layer $(n=3.34)$, a thin $0.2 \mu$ m layer of silica $(n=3.44)$ and a top layer of air $(n=1)$. Within this structure was placed a ridge of dimensions $1.1 \mu \mathrm{m} \times 2 \mu \mathrm{m}(\mathrm{n}=3.44)$. The nodes were distributed uniformly except at the material interfaces where two extra rows of nodes were added on either side of the interface (see Fig. 71(a)). For the graded-index method the permittivity was smoothed over this region of nodes.

The effective index of refraction for the first six modes of the ridge are shown in Table 9, with the eigenvectors from the FCM shown in Fig. 72. Comparison of the different results show a high degree of agreement for all of the methods. Numerical investigation determined that the results of the FCM for $n_{e f f}$ were quite robust with regards to node density and distribution and the specification of the gradient used for the graded-index method. 
Table 9: Comparison of effective index of refraction for the first six modes of the ridge waveguide. The s-FCM and g-FCM being the step-index FCM and the graded-index FCM, compared with results from Rsoft FemSIM and COMSOL MultiPhysics.

\begin{tabular}{c|c|c|c|c} 
Mode & s-FCM & g-FCM & Rsoft & COMSOL \\
\hline 1 & 3.388803 & 3.387818 & 3.388718 & 3.388699 \\
2 & 3.387870 & 3.387500 & 3.387854 & 3.387872 \\
3 & 3.333842 & 3.335003 & 3.333806 & 3.336465 \\
4 & 3.330638 & 3.330539 & 3.330954 & 3.334248 \\
5 & 3.330209 & 3.329923 & 3.330079 & 3.332082 \\
6 & 3.329599 & 3.327569 & 3.329560 & 3.331420
\end{tabular}
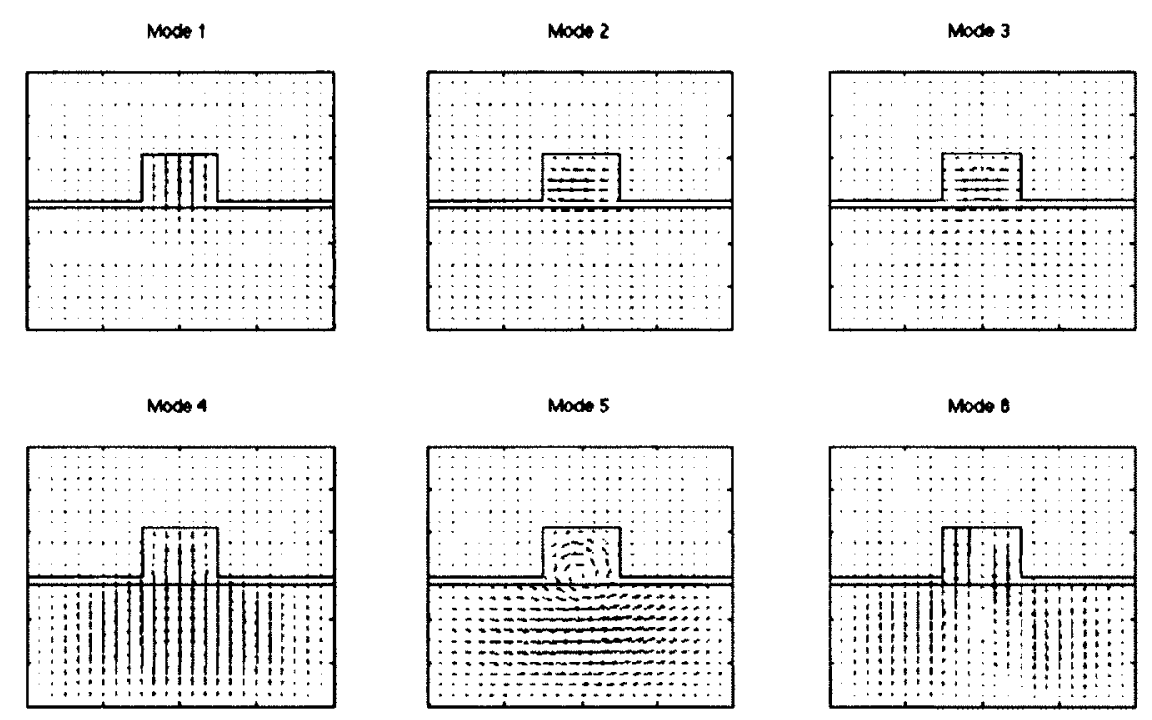

Figure 72: The first six modes of the ridge waveguide.

\section{Solid core step-index waveguide}

A second test with a solid core and a step-index, as described above in the convergence section, was performed and also compared with the two commercial solvers. The parameters and computational window of the solid core waveguide are shown in Fig. 66. This problem involves a waveguide with circular symmetry, as opposed to the 
Manhattan layout of the ridge waveguide. Therefore with this structure a radial distribution of nodes was used with a constant density throughout the region. At the interface between the core and the cladding additional nodes were inserted as for the ridge example.

Results for the first six modes of the solid core fiber are shown in Table 10, which show a high agreement with the commercial solvers on the same structure. The corresponding eigenvectors are seen in Fig. 67.

Table 10: Comparison of effective index of refraction for the first six modes of the step index solid core waveguide. The s-FCM and g-FCM being the step-index FCM and the graded-index FCM, compared with results from Rsoft FemSIM and COMSOL MultiPhysics.

\begin{tabular}{c|c|c|c|c} 
Mode & s-FCM & g-FCM & Rsoft & COMSOL \\
\hline 1 & 3.413099 & 3.413097 & 3.413093 & 3.413093 \\
2 & 3.413097 & 3.413097 & 3.413093 & 3.413093 \\
3 & 3.410550 & 3.410549 & 3.410527 & 3.410531 \\
4 & 3.410525 & 3.410524 & 3.410509 & 3.410512 \\
5 & 3.410525 & 3.410520 & 3.410509 & 3.410511 \\
6 & 3.410504 & 3.410499 & 3.410494 & 3.410495
\end{tabular}

The eigenvalue solutions for the step-index fiber have also been compared for various levels of symmetry, the entire domain, half symmetry and quarter symmetry. Results for all three situations and both graded and step methods are compared in Table 11. Values for the number of solved nodes and time for complete solution, including domain creation, matrix creation, and sparse eigenvector solving are also shown in the table. As can be seen, all configurations compare well with the commercial simulations and it was found that as with the ridge, the results were robust with respect to changes in the node distribution. For example, an orthogonal node distribution with a radially defined interface produced very similar numbers. 
Table 11: Comparison of effective index of refraction for the first six modes of the step fiber waveguide. The s-FCM and g-FCM being the step-index FCM and the graded-index FCM, compared with half and quarter symmetry using PEC and PMC boundaries. "Simulation time using a dual core iMac at $2.4 \mathrm{GHz}$.

\begin{tabular}{c|c|c|c|c|c|c} 
Mode & s-FCM & s-FCM $\frac{1}{2}$ & s-FCM $\frac{1}{4}$ & g-FCM & g-FCM $\frac{1}{2}$ & g-FCM $\frac{1}{4}$ \\
\hline 1 & 3.413099 & 3.413108 & 3.413107 & 3.413097 & 3.413098 & 3.413097 \\
2 & 3.413097 & 3.413097 & 3.413096 & 3.413097 & 3.413097 & 3.413097 \\
3 & 3.410550 & 3.410580 & 3.410576 & 3.410549 & 3.410550 & 3.410549 \\
4 & 3.410525 & 3.410540 & 3.410539 & 3.410524 & 3.410525 & 3.410523 \\
5 & 3.410525 & 3.410525 & 3.410525 & 3.410520 & 3.410525 & 3.410521 \\
6 & 3.410504 & 3.410501 & 3.410500 & 3.410499 & 3.410503 & 3.410499 \\
\hline \# of Nodes & 18445 & 9495 & 4840 & 18445 & 9495 & 4840 \\
\hline Time $^{*}(\mathrm{~s})$ & 38 & 15 & 7 & 29 & 13 & 7
\end{tabular}

\subsubsection{Two microstructured waveguides}

The previous two examples were relatively standard optical waveguides which are based on total internal reflection due to an optical difference between core and cladding. Presented in this section is the analysis of two microstructured optical fibers. The first is an idealized air core fiber suitable for applications where nonlinearities are to be kept to a minimum and is based on Bragg diffraction. The second is a structured fiber based on Photonic Crystal principles.

\section{Bragg diffraction air core}

The Bragg diffraction based waveguide, Fig. 73(a), used in this section is a simplifcation of the structure examined in [65] and is an idealized air core waveguide. The guide consists of a silica outer region at a radius of $19 \mu \mathrm{m}$ and an inner air core of $10 \mu \mathrm{m}$. The air core is surrounded by three annular shaped air regions having thickness $t_{\text {annular }}=2.3 \mu \mathrm{m}$ each separated by a thin silica ring of thickness $t_{\text {ring }}=0.2 \mu \mathrm{m}$. 

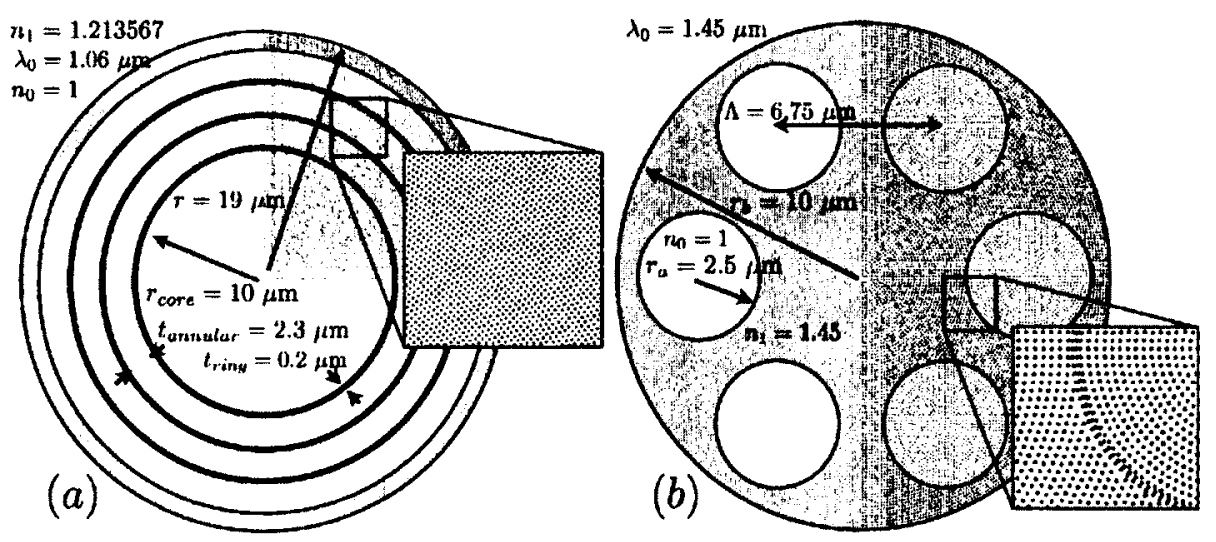

Figure 73: Parameters and dimensions for (a) a Bragg diffraction air core fiber and (b) a photonic crystal fiber with six circular air holes.

The index for the silica is calculated to be $n_{1}=1.213567$ and the index of air is $n_{0}=1$, with free space wavelength $\lambda_{0}=1.06 \mu \mathrm{m}$.

Radial meshing, with single row at the interface, is used and the s-FCM results were obtained using a full model and approximate spacing of $d_{\text {rad }} \approx 0.1 \mu \mathrm{m}$. The graded method results required a slightly finer mesh at the interfaces as it was found that a single node in the ring thickness of $0.2 \mu \mathrm{m}$ was not sufficient to capture the sharp index change from air-silica-air. Thus, a spacing of $d_{\text {rad }} \approx 0.066 \mu \mathrm{m}$ was used for the g-FCM results and quarter symmetry was also needed to reduce the computational window size resulting from the added nodes. The first six modes, Fig. 74, are compared in Table 12 for the two methods and two commercial solvers, all in very good agreement.

\section{Photonic crystal fibers}

Finally, a complex microstructured air hole waveguide is also examined using the FCM and compared with the commercial solvers. The structure is a photonic crystal fiber with six circular air holes with parameters and dimensions seen in Fig. 73(b), as published in [65]. Radial meshing was used for all seven discs; one primary disc 
Table 12: Comparison of effective index of refraction for the first six modes of the Bragg diffraction air core structure. The s-FCM and g-FCM being the step-index FCM and the graded-index FCM, compared with results from Rsoft FemSIM and COMSOL MultiPhysics.

\begin{tabular}{c|c|c|c|c} 
Mode & s-FCM & g-FCM & Rsoft & COMSOL \\
\hline 1 & 0.999111 & 0.998704 & 0.999107 & 0.999109 \\
2 & 0.999111 & 0.999370 & 0.999107 & 0.999109 \\
3 & 0.997792 & 0.997788 & 0.997787 & 0.997788 \\
4 & 0.997750 & 0.997495 & 0.997742 & 0.997744 \\
5 & 0.997750 & 0.997498 & 0.997742 & 0.997744 \\
6 & 0.997712 & 0.997268 & 0.997694 & 0.997704
\end{tabular}

and the 6 inset air holes, with triple row interface used for boundaries.

The fundamental quasi-degenerate modes of the structure are compared for the two methods and results shown in Table 13, and the modes shown in Fig. 75. Again, the two FCM solutions give highly accurate results in agreement with the compared methods.

Table 13: Comparison of effective index of refraction for the first six modes of the air hole waveguide. The s-FCM and g-FCM being the step-index FCM and the graded-index FCM, compared with results from Rsoft FemSIM and COMSOL MultiPhysics.

\begin{tabular}{c|c|c|c|c} 
Mode & s-FCM & g-FCM & Rsoft & COMSOL \\
\hline 1 & 1.445402 & 1.445411 & 1.445394 & 1.445395 \\
2 & 1.445400 & 1.445409 & 1.445394 & 1.445395 \\
3 & 1.438605 & 1.438635 & 1.438581 & 1.438582 \\
4 & 1.438384 & 1.438409 & 1.438355 & 1.438358 \\
5 & 1.438466 & 1.438494 & 1.438442 & 1.438440 \\
6 & 1.438466 & 1.438490 & 1.438440 & 1.438440
\end{tabular}

As with the step-fiber example, the air hole fiber has a physical symmetry which can be used to reduce the domain size and computational load. The three domain 

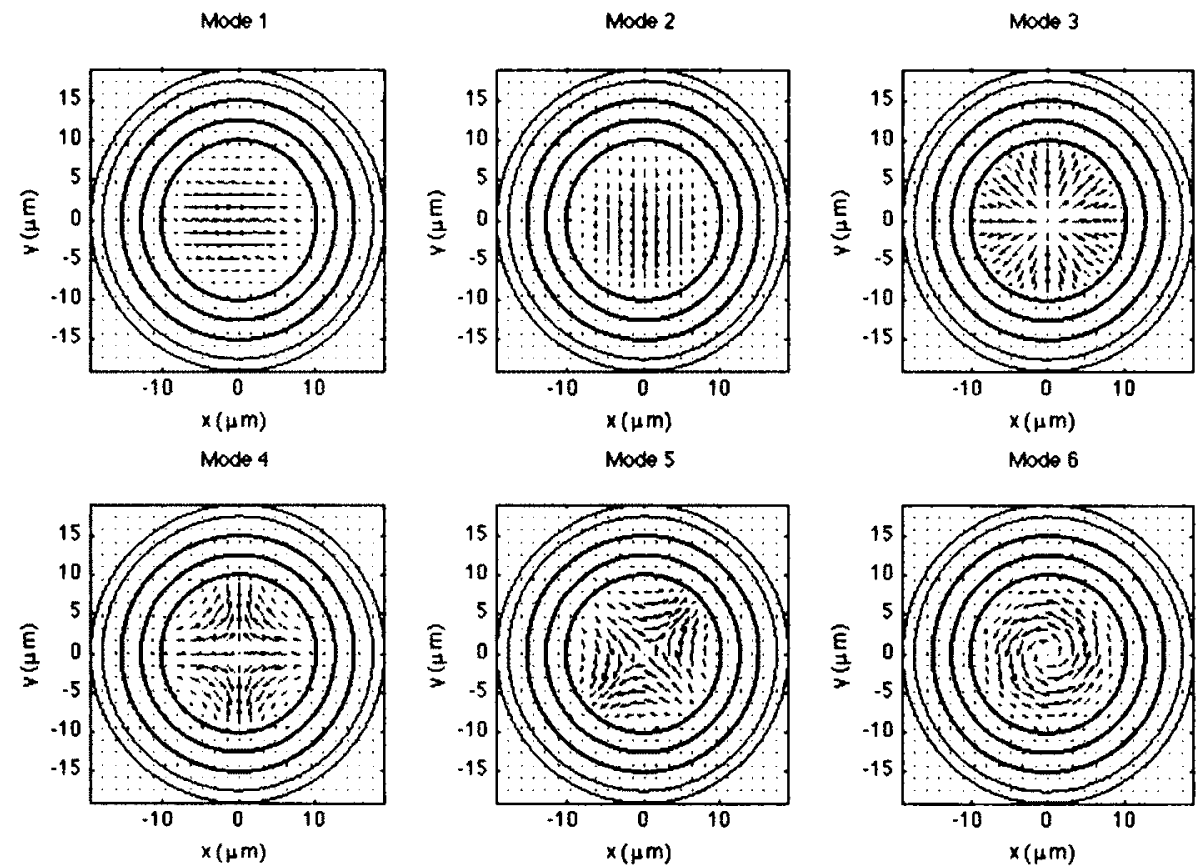

Figure 74: The first six modes of the Bragg diffraction air core structure.
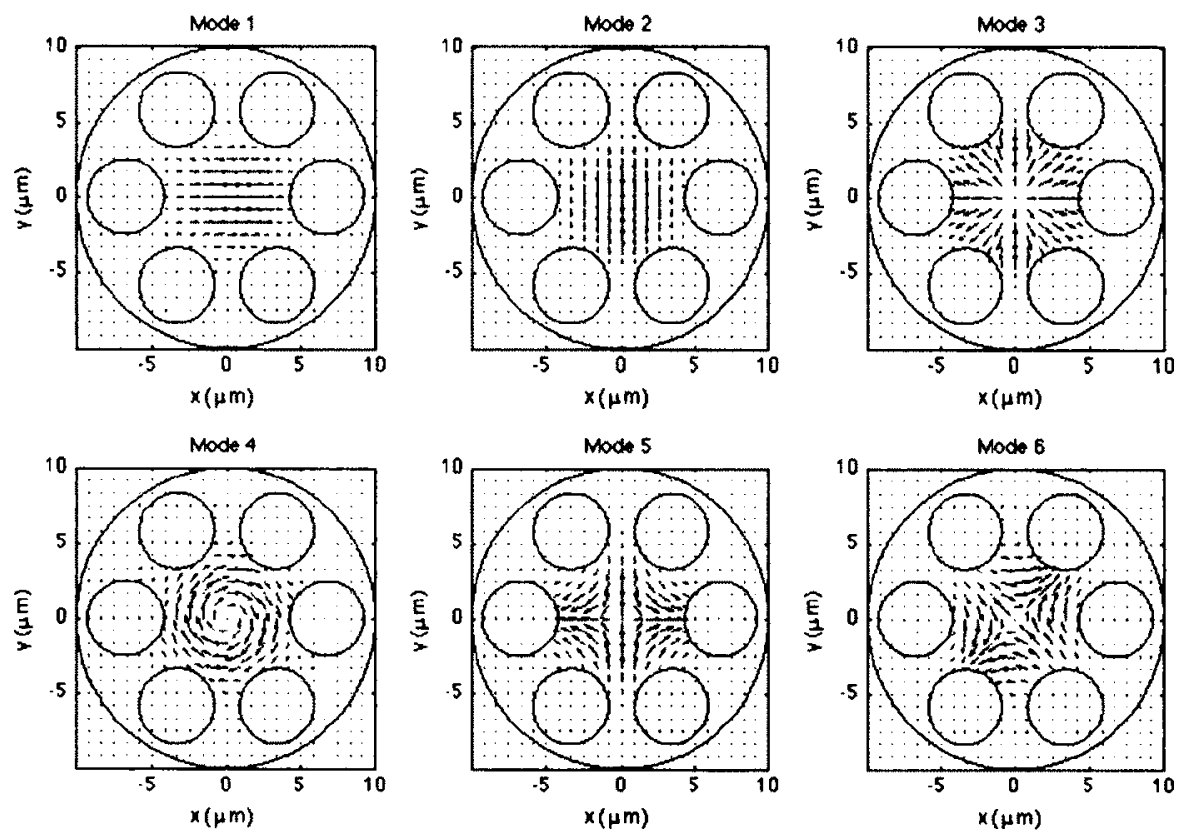

Figure 75: The first six modes of the circular air hole photonic crystal fiber. 
sizes, full, half, and quarter, are compared in Table 14 along with the full solution time and number of nodes in the domain.

Table 14: Comparison of effective index of refraction for the first six modes of the air hole waveguide. The s-FCM and g-FCM being the air-hole FCM and the graded-index FCM, compared with half and quarter symmetry using PEC and PMC boundaries.

\begin{tabular}{c|c|c|c|c|c|c} 
Mode & s-FCM & s-FCM $\frac{1}{2}$ & s-FCM $\frac{1}{4}$ & g-FCM & g-FCM $\frac{1}{2}$ & g-FCM $\frac{1}{4}$ \\
\hline 1 & 1.445402 & 1.445404 & 1.445404 & 1.445411 & 1.445402 & 1.445412 \\
2 & 1.445400 & 1.445378 & 1.445378 & 1.445409 & 1.445391 & 1.445411 \\
3 & 1.438605 & 1.438569 & 1.438569 & 1.438635 & 1.438610 & 1.438633 \\
4 & 1.438384 & 1.438366 & 1.438365 & 1.438409 & 1.438363 & 1.438417 \\
5 & 1.438466 & 1.438442 & 1.438441 & 1.438494 & 1.438471 & 1.438498 \\
6 & 1.438466 & 1.438441 & 1.438440 & 1.438490 & 1.438445 & 1.438491 \\
\hline \# of Nodes & 21181 & 10625 & 5454 & 21181 & 10625 & 5454 \\
\hline Time (s) & 40 & 16 & 8 & 36 & 16 & 8
\end{tabular}

A further test of the photonic crystal fiber is to investigate the dispersion properties of the fiber in the region of operation around its operating wavelength. The dispersion parameter is based on the second derivative of the effective index with respect to the wavelength and is calculated as [65]

$$
\text { Dispersion }=-\frac{\lambda}{c} \frac{\partial^{2}}{\partial \lambda^{2}}\left[\Re\left(n_{e f f}\right)\right]
$$

For simplicity, in this comparison the index of refraction for the materials is kept constant with a varying wavelength. A more rigorous fiber characterization would include the variation of the index of refraction as a function of the wavelength. The dispersion of the fundamental mode and the third mode from Fig. 75 for the photonic crystal fiber is compared using the FCM against the results from COMSOL MultiPhysics and seen in Fig. 76. The results show that the FCM is in very good 


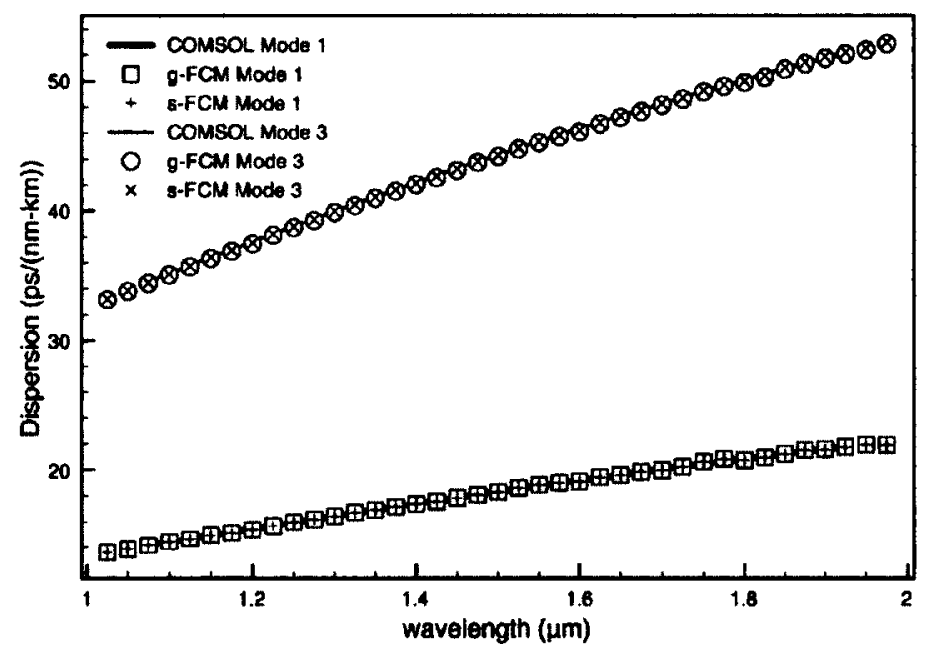

Figure 76: Dispersion parameter comparison between COMSOL MultiPhysics and the FCM for modes 1 and 3 of the photonic crystal fiber.

agreement with the dispersion found using a commercial solver.

\subsubsection{Metallic structures}

Optoelectronic devices, as described in Chapter 5.6.2, often have metal routing near to the waveguide. The metal acts as a source of loss if it is placed close enough to the waveguide that the tail of the optical field extends into the metal and is absorbed. The metal has a non zero imaginary component to its refractive index which provides the loss mechanism for the mode solver.

A transverse cross section of the ridge waveguide discussed in the optoelectronic section along with its properties and dimensions is shown in Fig. 77. The real effective index and loss of the first two fundamental modes of the waveguide, found using the s-FCM and g-FCM, are calculated and compared with commercial solvers in Table 15.

The real component of the effective index of refraction differed between the graded method and COMSOL by less than $0.1 \%$ and $0.5 \%$ for the first and second modes 


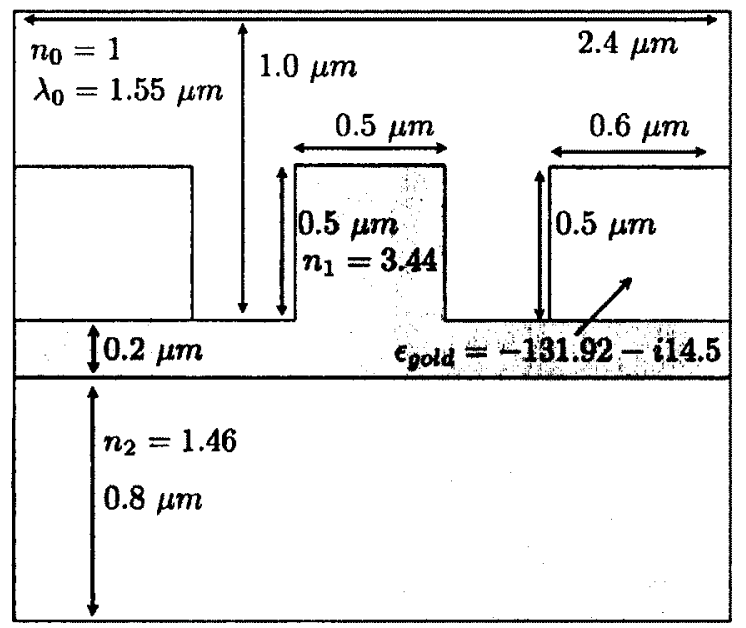

Figure 77: Parameters and dimensions for the lossy ridge waveguide with gold on either side of the ridge.

respectively. The stitched method has smaller error with $0.001 \%$ and $-0.02 \%$, which is less than the error in the modes returned by Rsoft compared to COMSOL, which are $-0.09 \%$ and $-0.1 \%$ respectively. The imaginary, lossy, component of the effective index found using the g-FCM is significantly smaller than that found using the commercial solvers. The error in the imaginary component of the s-FCM effective indices with respect to those found by COMSOL is, however, on the order of the differences between Rsoft and COMSOL. Further study of lossy waveguides and the FCM is necessary.

\subsection{Leaky boundary conditions}

The previous examples above have attempted problems for which Dirichlet boundary conditions were used, producing pure guided modes. However, of significant interest is the solution of "leaky" waveguides with lossy modes characterized by a complex effective index. The simulation of leaky modes is problematic for finite region numerical simulators due to the difficulty in defining an appropriate boundary condition. 
Table 15: Comparison of the real component of the effective index of refraction for the first two modes of the lossy ridge waveguide. The s-FCM and g-FCM being the step-index FCM and the graded-index FCM, compared with results from Rsoft FemSIM and COMSOL MultiPhysics. Second, the loss component in $\mathrm{dB} / \mathrm{cm}$ of the waveguide compared with the same solvers.

\begin{tabular}{c|c|c|c|c}
$\begin{array}{c}\text { Mode } \\
\operatorname{Re}\left(n_{\text {eff }}\right)\end{array}$ & s-FCM & g-FCM & Rsoft & COMSOL \\
\hline 1 & 3.055450 & 3.064916 & 3.052639 & 3.055567 \\
2 & 3.024470 & 3.039217 & 3.021897 & 3.025161 \\
\hline \hline Mode & \multicolumn{3}{|c}{} \\
$\operatorname{Im}\left(n_{\text {eff }}\right)$ & s-FCM & g-FCM & Rsoft & COMSOL \\
\hline 1 & $7.4 \mathrm{E}-5$ & $6.8 \mathrm{E}-6$ & $6.2 \mathrm{E}-5$ & $4.1 \mathrm{E}-5$ \\
2 & $1.5 \mathrm{E}-4$ & $2.5 \mathrm{E}-5$ & $5.5 \mathrm{E}-4$ & $3.7 \mathrm{E}-4$
\end{tabular}

Two approaches have been used in the past and applied to FD and FEM approaches.

These are: 1) transparent boundaries which approximates the absorbing boundary with a non-linear Neumann boundary condition [65], and 2) perfectly matched layers which adds an absorbing layer (using a complex optical index) to the boundary [66]. The details of how these methods can be incorporated into the FCM are presented in this chapter.

\subsubsection{Transparent boundary condition}

The transparent boundary conditions (TBC) which are employed are $1^{\text {st }}$-order Bayliss-Gunzburger-Turkel-like (BGT-like) TBC [65] using a nonlinear Neumann BC 
which places the following boundary restrictions:

$$
\begin{aligned}
\frac{\partial H_{x}}{\partial x} & =-\hat{r} \bullet \hat{x}\left(j \kappa_{r, x}+\frac{1}{2 r}\right) H_{x} \\
\frac{\partial H_{x}}{\partial y} & =-\hat{r} \bullet \hat{y}\left(j \kappa_{r, x}+\frac{1}{2 r}\right) H_{x} \\
\frac{\partial H_{y}}{\partial x} & =-\hat{r} \bullet \hat{x}\left(j \kappa_{r, y}+\frac{1}{2 r}\right) H_{y} \\
\frac{\partial H_{y}}{\partial y} & =-\hat{r} \bullet \hat{y}\left(j \kappa_{r, y}+\frac{1}{2 r}\right) H_{y}
\end{aligned}
$$

with $r$ the radius at the boundary, $\hat{r}$ the normal at the boundary, and $\kappa$ being:

$$
\begin{aligned}
\kappa_{r, x} & =k_{0} \sqrt{n_{x x}^{2}-n_{e f f}^{2}} \\
\kappa_{r, y} & =k_{0} \sqrt{n_{y y}^{2}-n_{e f f}^{2}} .
\end{aligned}
$$

As the boundary condition includes the eigenvalue that is to be found, the eigenvalue equation, Eq. (133) becomes,

$$
\mathbf{A X}+\mathbf{A}_{\lambda} \mathbf{X}+\mathbf{B}(\lambda) \mathbf{I X}=\lambda \mathbf{X}
$$

where $\mathbf{A}_{\lambda}$ and $\mathbf{B}(\lambda)$ are specified by Eq. (148) and (149).

Due to the nonlinear nature of this equation it is necessary to iterate until the solution converges. During this iteration the matrix being decomposed, $\mathbf{A}$, changes at each step. Searching for modes which are either degenerate or are approximately equal in eigenvalue to other modes can cause the eigenvalue solver to return either the wrong mode or on occasion a mixture of two modes, which can cause convergence issues.

To avoid this problem a second level of iteration is added to the procedure. The first step is to implement Dirichlet boundary conditions on the problem and to solve 
for as many modes as needed. These are the initial or 'seed' modes, as the leaky modes will have similar eigenvectors and values. With the TBC implemented, any mode of interest can then be further refined using RQI inside the iteration on $n_{E f f}$ for the TBC. In effect, the approximate mode is chosen and given as the seed values for RQI, with a TBC. This is iterated until a new eigenvector-value pair is found, which is then used as the new guess for the TBC. RQI is then performed again to find the next eigen pairs. This procedure ensures that the mode of interest is always kept, without any mode hopping or mixing which can be produced using solely an eigenvalue solver.

\section{Convergence issues}

The boundary conditions shown in Eq. (148) for $\mathbf{H}_{\mathbf{x}}$ are implemented in a discrete form as:

$$
\begin{aligned}
& \left.\mathbf{N}_{\mathbf{x}}\right|_{i} \cdot \mathbf{H}_{\mathbf{x}}+\cos \phi\left(j \kappa_{r, x}+\frac{1}{2 r}\right)\left[\mathbf{H}_{\mathbf{x}}\right]_{i}=0 \\
& \left.\mathbf{N}_{\mathbf{y}}\right|_{i} \cdot \mathbf{H}_{\mathbf{x}}+\sin \phi\left(j \kappa_{r, x}+\frac{1}{2 r}\right)\left[\mathbf{H}_{\mathbf{x}}\right]_{i}=0
\end{aligned}
$$

where $\left.\mathbf{N}_{\mathbf{x}}\right|_{i}$ and $\left.\mathbf{N}_{\mathbf{y}}\right|_{i}$ are i'th rows of the matrices $\mathbf{N}_{\mathbf{x}}$ and $\mathbf{N}_{\mathbf{y}}$. These vector operators determine the spatial derivative of the solution at the $\mathbf{i}$ 'th boundary node. $\left[\mathbf{H}_{\mathbf{x}}\right]_{i}$ is the field at the boundary node $i, \phi$ the normal to the boundary interface as measured counter-clockwise from the positive $\mathrm{x}$-axis, and $r$ the radius at the boundary. These two equations must be enforced for every boundary node of the $\mathrm{TBC}$, and this is accomplished by summing them.

A problem occurs, however, with the changing sign of cos, sin and the derivatives in each of the four quadrants in a circular domain. In quadrant one, $0^{\circ} \leq \phi \leq 90^{\circ}$, the values are all positive and the equations are summed. In quadrants two and four 
the sin or cos values as well as the corresponding derivatives experience a change in sign. The result is that the two equations are no longer summed but are subtracted from each other. This subtraction creates a numerical problem as, for example, at $45^{\circ}$ off the axis a subtraction exactly cancels out both equations giving $0=0$.

Tests using this formulation of the boundary condition showed a large amount of instability in the imaginary component of the effective index $\left(\Im\left(n_{e f f}\right)\right)$. Fluctuations in $\Im\left(n_{\text {eff }}\right)$ over orders of magnitude were obtained for identical structures having varying node densities or varying $\mathrm{BC}$ radii. To account for this problem a different formulation of the boundary condition was used; the first line of Eq. (151) is multiplied by -1 in quadrants two and three, $90^{\circ} \leq \phi \leq 270^{\circ}$. The second line of $(151)$ is multiplied by -1 in quadrants three and four, $180^{\circ} \leq \phi \leq 360^{\circ}$. This modification ensures that the equations are always summed and remain symmetric in all four quadrants of the unit circle. With this formulation in place it was found that the effective index stabilized and became relatively independent of any changes in the node densities or the outer radius. As the conditions at the boundary in all four quadrants are now exactly the same, the solutions enforcing this fix are dubbed 'symmetric'. This enforced boundary symmetry refers to the implementation of the boundary condition only and implies nothing about the presence of geometric symmetry in the waveguide structure. Results for the two formulations are presented for the examined fibers in Section 7.5.

\subsubsection{Perfectly matched layer}

The perfectly matched layer (PML) is the second boundary condition used to solve for leaky modes. The method truncates the unbounded electromagnetic problem by adding an absorbing layer to the edge of the simulation domain. This layer performs a change in variable from the real domain into the complex, based on the depth into the 


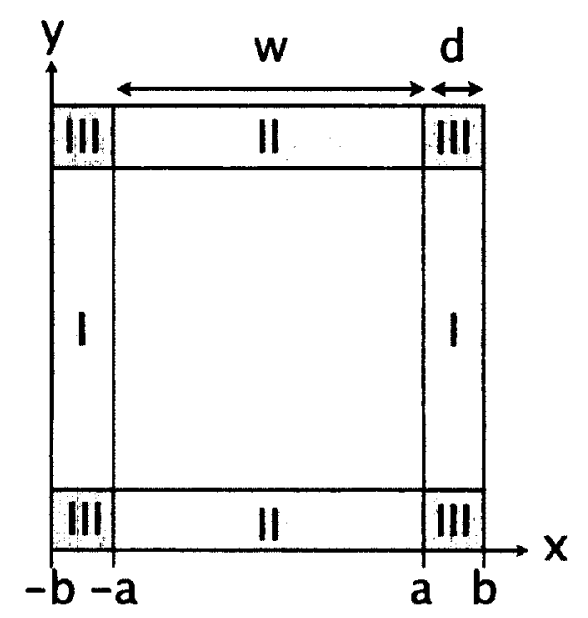

Figure 78: Three different regions of the PML.

PML. There are three differing regions for this method, corresponding to the variable or combination of variables being changed, and they are shown in Fig. 78. The PML will be described for area I, however, it is equally applicable to areas II and III using the appropriate coordinates, or superposition of coordinates [67].

The depth into area I corresponds to the $x$ coordinate, which will undergo the following modification to its derivative:

$$
\frac{\partial}{\partial x}^{\prime}=\frac{1}{s} \frac{\partial}{\partial x}
$$

with the stretching variable $s=1$ for $-a<x<a$ and when $|x|>a$ we have

$$
s=1-i \frac{(m+1) \lambda}{4 \pi n d} \ln \left(\frac{1}{R}\right)\left(\frac{\rho}{d}\right)^{m},
$$

with $\lambda$ the free space wavelength, $n$ the refractive index of the medium, $d$ being the depth of the PML, $\rho$ the distance into the PML, $m=2$ for a parabolic conductivity, and $R$ the theoretical reflection coefficient [67]. Thus if $x$ is in the interior of the domain, $s=1$ and Eq. (129) remains unchanged as expected. For nodes inside 
region I, Eq. (129) becomes:

$$
\begin{aligned}
& \frac{1}{s} \frac{\partial}{\partial x}\left(\frac{1}{s} \frac{\partial H_{x}}{\partial x}\right)+\frac{\partial^{2} H_{x}}{\partial y^{2}}+\left(\epsilon_{r} k_{0}^{2}-\beta^{2}\right) H_{x}=0 \\
& \frac{1}{s} \frac{\partial}{\partial x}\left(\frac{1}{s} \frac{\partial H_{y}}{\partial x}\right)+\frac{\partial^{2} H_{y}}{\partial y^{2}}+\left(\epsilon_{r} k_{0}^{2}-\beta^{2}\right) H_{y}=0
\end{aligned}
$$

Finally the boundary of the PML is terminated with a perfect magnetic conductor (PMC) for which,

$$
H_{\|}=0
$$

where $H_{\|}$is the magnetic field component parallel to the boundary.

Unlike the TBC, the use of the PML does not change the nature of the eigenvector equation and a simple solution for the eigenvectors and values is possible. However, it does introduce the possibility of cladding modes into the solutions, which are undesired modes confined to the cladding. These modes can be due to the larger cladding areas required by the PML.

A simple solution to the cladding modes is to search for a larger number of modes than is desired and ignore the modes which are of no interest. A more robust solution to the problem is to first obtain a rough set of solutions using Dirichlet boundary conditions, then select the mode of interest and use RQI with the PML boundary to iterate to the desired mode. This removes the need to find a large number of extra modes and ensures that the mode of interest is the one that is found.

\section{Free parameters in the PML}

A complication of the PML is that there are a number of free parameters that can be adjusted and which may affect the solution. The PML is said to have to be 'tuned' to the correct values to obtain a valid solution. These parameters are $w$, the width of 
the inner region before the PML begins, $d$, the depth of the PML and $R$ the reflection coefficient.

In principle, if a solution is said to be converged and stable, the results would be independent of perturbations in any of the free parameters. It can be found that by varying the reflection coefficient one can converge on a solution which is independent of $w$ and $d$, and stable for small variations in $R$.

Several articles address the value of $R$ and come to a variety of conclusions. A suggestion for an approximate value is found in [66] which gives $R \approx(\Delta x / \lambda)^{2}$. This approximation brings the value of the reflection $R$ to the same order as the errors introduced in the discretization process of an FD mesh. Another group, also using FD and analyzing similar waveguides to those in this paper, have found in [68] that using values of $R \approx 10^{-100}$ were appropriate and gave the most stable solution.

We have used a procedure to vary the three parameters until a result for $R$ is found which stabilizes the solution with $w$ and $d$. For each PML solution the values of the three free parameters will be given.

\subsection{Leaky mode results}

For the leaky mode examples, the eigenvalues and vectors are typically found using RQI in either Matlab [48] or Octave [59] and are based upon initial approximate solutions using the 'eigs' function which calls an appropriate function from Arpack. For Dirichlet or PML boundary conditions this is the simple RQI procedure as the equation is linear. However, for the TBC case, $\mathbf{A}$ is a function of the solution through $\lambda$ and a double iteration scheme is used as described above.

The primary challenge present in the implementation of leaky mode solving is the 
accurate and robust determination of the imaginary part of the effective index of propagation. The magnitude of this quantity is generally $4-8$ orders of magnitude smaller than the real part of the index. As any finite boundary condition is an approximation which involves a number of parameters, it is desirable that the solution be insensitive to any free parameters. For the TBC methodology, the only free parameter is the size (radius) of the domain. The PML, however, has three free parameters $(R, d$ and $w)$.

In the following sections two complex waveguides will be used to first determine the robustness of two methods to parameter variation and also to present a direct comparison of the value of complex index to commercial mode solvers. In all situations the full fiber is analyzed, without using any geometric symmetries to reduce the domain size.

\subsubsection{Air hole waveguide}

The first structure studied using absorbing boundary conditions is the photonic crystal fiber as seen above in Fig. 73(b). The point distribution shown is typical, but will vary for specific cases due to variation in the geometry and PML parameters. For the structure shown, the average distance between points is $0.125 \mu \mathrm{m}$ in the structure with an outwardly varying distance, expanding to $0.5 \mu \mathrm{m}$ at the boundary edge.

The first leaky case studied was for a TBC where the outer domain diameter was varied to observe its effect on the small imaginary component of the effective index for the fundamental mode. This is shown in Fig. 79 for both with and without the symmetry enforcement described previously. Prior to the enforcement of symmetry the solution is highly variable with respect to the diameter, however, the use of symmetry produces a stable response.

To initially characterize the PML, formulation tests were undertaken which iterated over the three variable parameters width, $w$, depth, $d$ and reflectance, $R$. The 


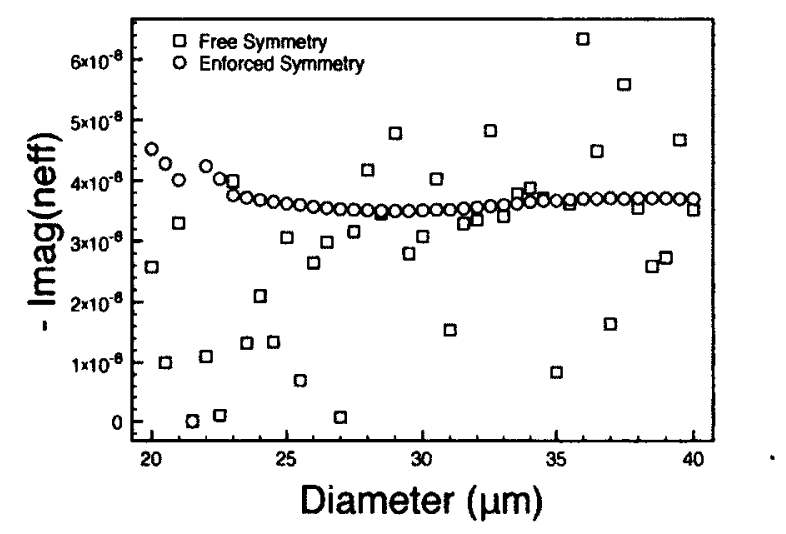

Figure 79: Imaginary component of the index of refraction for the fundamental mode of the air hole fiber with a $\mathrm{TBC}$ at a varying diameter.

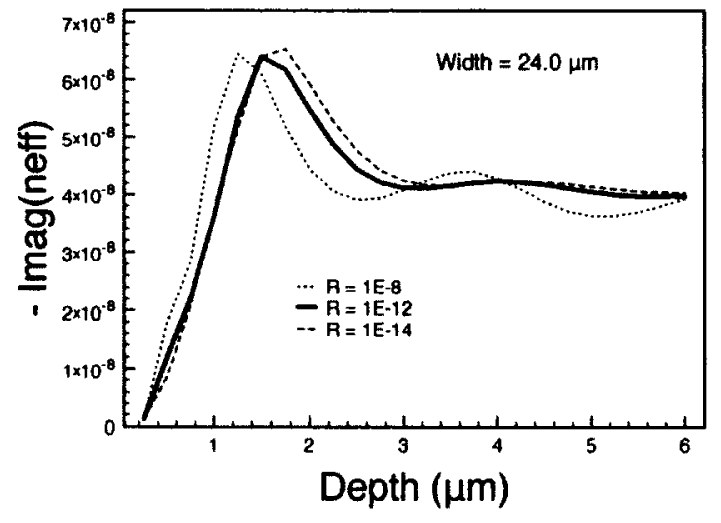

(a)

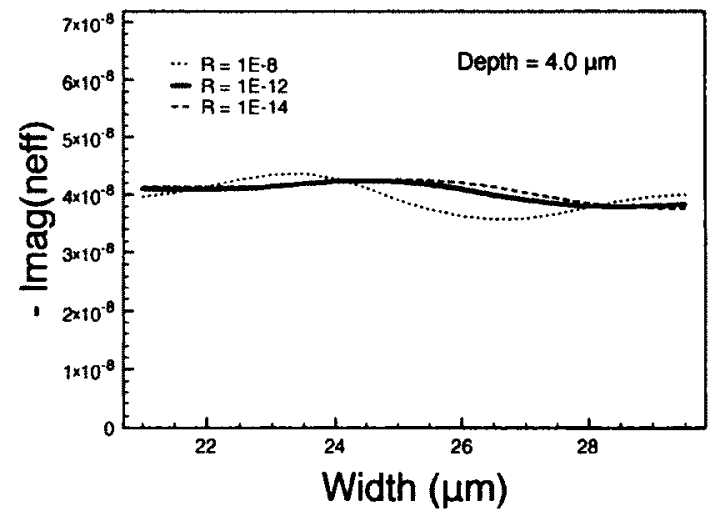

(b)

Figure 80: Imaginary component of the index of refraction for the fundamental mode of the air hole fiber with a PML at (a) varying depth, and (b) varying width. 
variation in the imaginary component of the fundamental mode with respect to these three parameters is shown in Fig. 80. For this case of a low loss fiber, the behaviour of imaginary $n_{e f f}$ is smooth and predictable. The solution shows minimal variation for reflectance values of $R \approx 10^{-12}$ with $d \geq 4 \mu \mathrm{m}$ and $w$ large enough to encompass the structure.

With appropriate parameters for both of the boundary condition methods, the effective indices of the first six modes (presented in Fig. 81) are compared with a previously published FEM solution [65] in Table 16. All of the methods are very close with respect to the real part of $n_{\text {eff }}$ and the imaginary part is also in good agreement, considering the very small magnitude.

Table 16: Comparison of effective index of refraction for the first six modes of the circular air holephotonic crystal fiber. The TBC and PML solutions are compared with results from a previously published FEM simulation.

\begin{tabular}{c|c|c|c|c|c|c} 
Mode & \multicolumn{2}{|c|}{ FCM-TBC } & \multicolumn{2}{c|}{ FCM-PML } & \multicolumn{2}{c}{ FEM } \\
& $\operatorname{Re}\left(n_{\text {eff }}\right)$ & $-\operatorname{Im}\left(n_{\text {eff }}\right)$ & $\operatorname{Re}\left(n_{\text {eff }}\right)$ & $-\operatorname{Im}\left(n_{\text {eff }}\right)$ & $\operatorname{Re}\left(n_{\text {eff }}\right)$ & $-\operatorname{Im}\left(n_{\text {eff }}\right)$ \\
\hline 1 & 1.445409 & $3.28 \mathrm{E}-8$ & 1.445453 & $3.96 \mathrm{E}-8$ & 1.445393 & $4.11 \mathrm{E}-8$ \\
2 & 1.445411 & $3.21 \mathrm{E}-8$ & 1.445474 & $4.00 \mathrm{E}-8$ & 1.445394 & $4.12 \mathrm{E}-8$ \\
3 & 1.438635 & $5.70 \mathrm{E}-7$ & 1.438772 & $8.62 \mathrm{E}-7$ & 1.438576 & $3.97 \mathrm{E}-7$ \\
4 & 1.438490 & $1.07 \mathrm{E}-6$ & 1.438691 & $1.42 \mathrm{E}-6$ & 1.438442 & $7.13 \mathrm{E}-7$ \\
5 & 1.438494 & $1.01 \mathrm{E}-6$ & 1.438676 & $1.13 \mathrm{E}-6$ & 1.438438 & $7.11 \mathrm{E}-7$ \\
6 & 1.438409 & $1.50 \mathrm{E}-6$ & 1.438544 & $1.50 \mathrm{E}-6$ & 1.438362 & $1.03 \mathrm{E}-6$ \\
\hline
\end{tabular}

The TBC case involves solving a nonlinear eigenvalue problem, however it was found that one entire eigenvalue-eigenvector solution required approximately $25 \%$ less computational time as a similarly sized linear PML solution. This is due to the extra iterations that the PML solution required to before converging on a solution. Additionally, the full PML solution requires many complete iterations to determine the appropriate parameters. Obtaining the PML solutions requires significantly more 

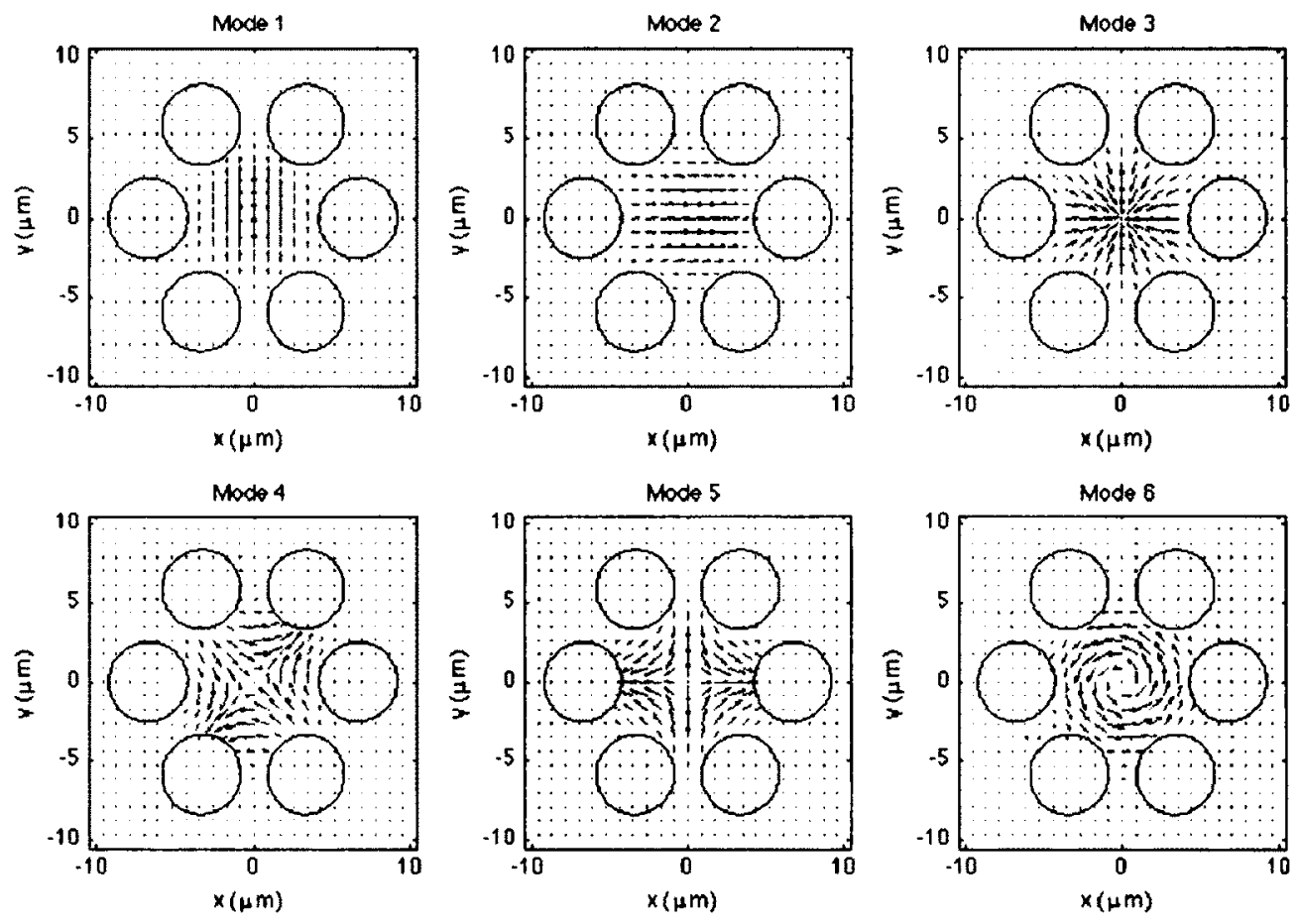

Figure 81: The first six modes of the circular air holephotonic crystal fiber using a square PML.

time and effort than the use of a TBC.

\subsubsection{Annular air hole waveguide}

A more complex air hole waveguide with non-circular microstructured components has also been examined and compared with an FEM solution [65]. The structure is a pure silica core with three annular shaped air holes (see Fig. 82) and is a much more lossy fiber than the previous example.

As with the previous fiber the TBC solution utilized, the symmetric boundary conditions and the PML parameters have all been tuned to a stable solution. The comparison of the fundamental modes imaginary index for a varying diameter using 


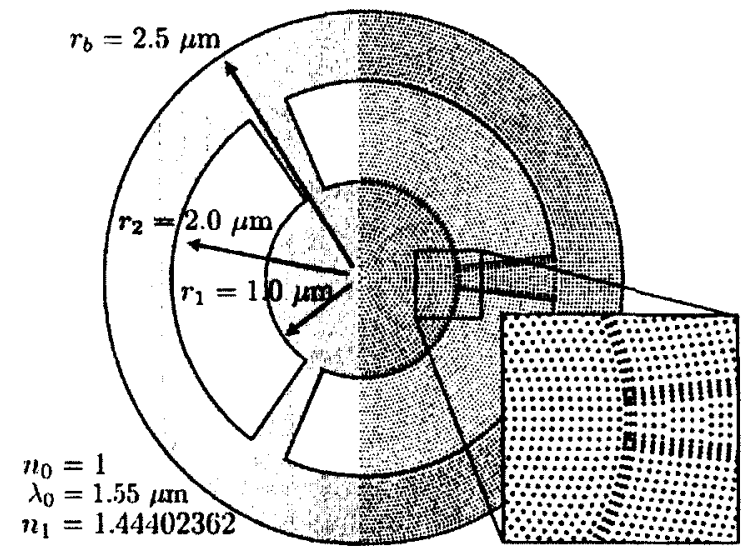

Figure 82: Parameters and dimensions for the annular ring air hole fiber.

TBC boundaries is shown in Fig. 83. The improvement obtained by enforcing numerical symmetry can be clearly seen, with a stable response seen for any boundary diameter over $6 \mu \mathrm{m}$. It should be noted that the magnitude of the imaginary part of $n_{\text {eff }}$ for this fiber is 1000 times larger than the previous case.

The tuning of the PML for this fiber can be seen in Fig. 84 showing the effect of varying the width, depth and reflectance parameters. Although the plots show a single width for varying depth, and a single depth for varying width, a full parameter search was performed and the values closest to convergence are shown. Similarly for the reflectance parameter, a large search range involving many orders of magnitude was performed, showing only those near convergence. For this relatively lossy structure it can be seen that for the appropriate choice of $R=1 \mathrm{E}-3$ the solution is stable for depths over $0.8 \mu \mathrm{m}$. It is, however, less well behaved for variation in width, although its variation is bounded. This variation may be due to the large amount of loss combined with a threefold symmetry in the fiber which is bounded by a rectangular PML. The large variation with respect to the width was also noticed when obtaining similar solutions using commercial mode solvers. The solution parameters for the PML were chosen to minimize the solution sensitivity and were $R \approx 1 \mathrm{E}-3$ and $d \geq$ 


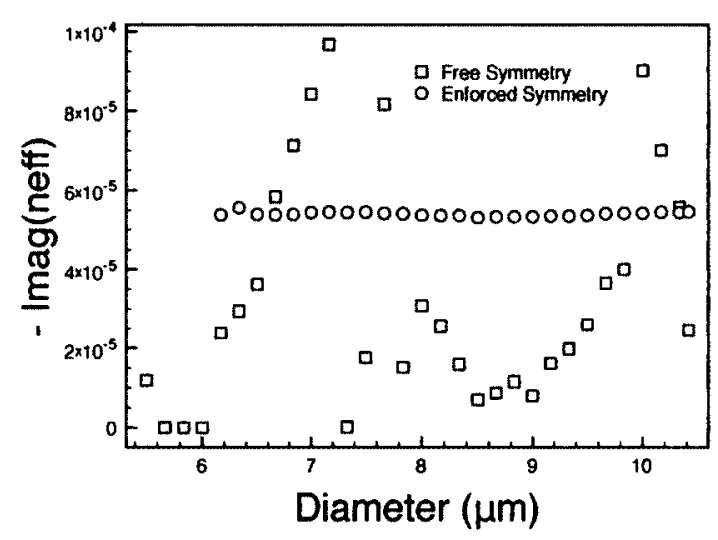

Figure 83: Imaginary component of the index of refraction for the fundamental mode of the annular air hole fiber with a TBC at a varying diameter.

$0.8 \mu \mathrm{m}$ and an appropriate $w$ to fully encompass the air hole structure.

The first six modes for the fiber are shown in Figure 85. The modes for this fiber are quite leaky, with the published results showing the fundamental mode having a loss of $17.44 \mathrm{~dB} / \mathrm{cm}$. The previous air-hole structure has a loss of $0.0155 \mathrm{~dB} / \mathrm{cm}$ considerably less than this structure. Results comparing the effective refractive indices for various modes are shown in Table 17 and show good agreement with the other two methods. The FEM mode solutions show near-degeneracy amongst several pairs of eigenmodes (1:2 and 4:5), the FCM TBC/PML solutions also show these modes to be near degenerate, however, the difference is somewhat larger. The real part of the index for the TBC and PML methods differ by $0.51 \%$ and $0.31 \%$ respectively. It should be noted that the FEM solutions show a much closer level of degeneracy. It is believed that the splitting of the modes when using the FCM is due to the use of a graded material transition and is the subject of ongoing work. 


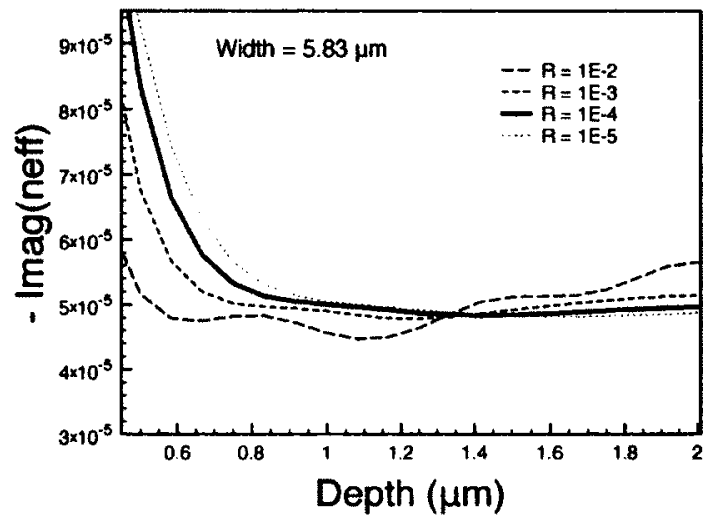

(a)

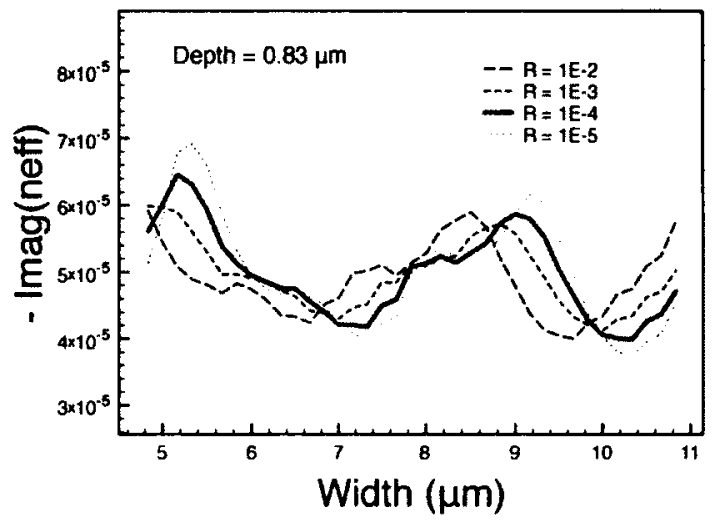

(b)

Figure 84: Imaginary component of the index of refraction for the fundamental mode of the annular air hole fiber with a PML at (a) varying depth, and (b) varying width.
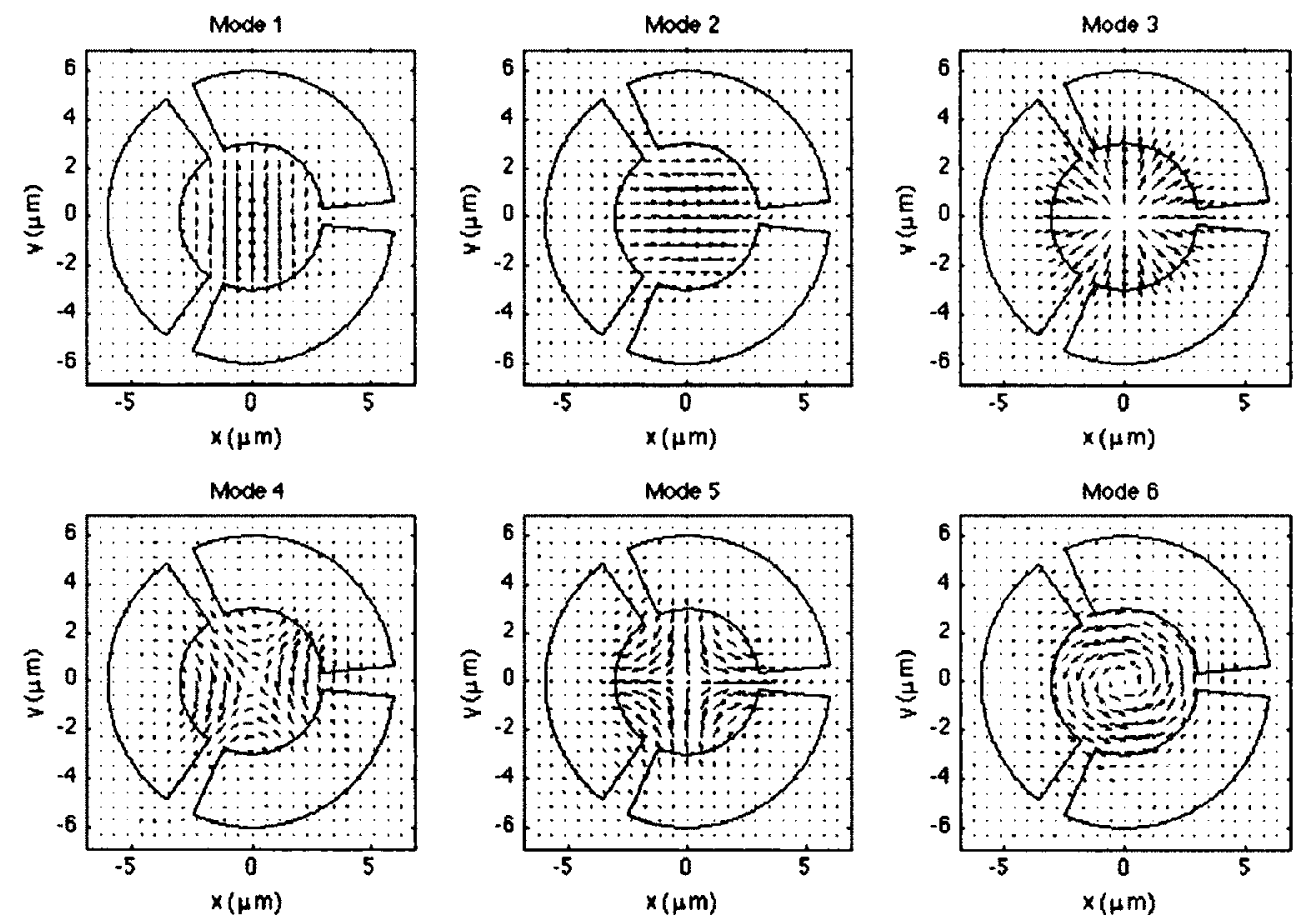

Figure 85: The first six modes of the annular ring air hole fiber using a square PML. 
Table 17: Comparison of effective index of refraction for the first six modes of the annular ring air hole fiber. The TBC and PML solutions compared with results from a FEM simulation.

\begin{tabular}{c|c|c|c|c|c|c}
\multirow{2}{*}{ Mode } & \multicolumn{2}{|c|}{ FCM-TBC } & \multicolumn{2}{c|}{ FCM-PML } & \multicolumn{2}{c}{ FEM } \\
& $\operatorname{Re}\left(n_{\text {eff }}\right)$ & $-\operatorname{Im}\left(n_{\text {eff }}\right)$ & $\operatorname{Re}\left(n_{\text {eff }}\right)$ & $-\operatorname{Im}\left(n_{\text {eff }}\right)$ & $\operatorname{Re}\left(n_{\text {eff }}\right)$ & $-\operatorname{Im}\left(n_{\text {eff }}\right)$ \\
\hline 1 & 1.355188 & $4.63 \mathrm{E}-5$ & 1.355852 & $4.97 \mathrm{E}-5$ & 1.35580 & $4.95 \mathrm{E}-5$ \\
2 & 1.355811 & $4.80 \mathrm{E}-5$ & 1.355780 & $5.33 \mathrm{E}-5$ & 1.35581 & $4.96 \mathrm{E}-5$ \\
3 & 1.239674 & $4.57 \mathrm{E}-4$ & 1.239467 & $4.29 \mathrm{E}-4$ & 1.23950 & $5.67 \mathrm{E}-4$ \\
4 & 1.216690 & $1.10 \mathrm{E}-3$ & 1.216898 & $1.09 \mathrm{E}-3$ & 1.21480 & $1.11 \mathrm{E}-3$ \\
5 & 1.209794 & $1.37 \mathrm{E}-3$ & 1.213086 & $1.12 \mathrm{E}-3$ & 1.21479 & $1.11 \mathrm{E}-3$ \\
6 & 1.215655 & $1.78 \mathrm{E}-3$ & 1.217210 & $1.74 \mathrm{E}-3$ & 1.21655 & $1.96 \mathrm{E}-3$ \\
\hline
\end{tabular}

\subsection{Adaptive node refining}

As previously discussed in the convergence and node mapping section, having appropriate node placement for a model can both increase the solution accuracy and decrease the total number of points necessary for the solution. If the model is not too complex, or the approximate solution is known a priori, designers can tailor the point distributions as needed. This, however, can be a time consuming task and would ideally be accomplished in an automated manner.

Adaptive node refining is a technique which allows an automated node placement in locations of high solution gradient. The method begins with a coarse node distribution and finds a solution to the given PDE. With an approximate solution the gradients can be trivially calculated using the derivative shape functions which have already been created during the FCM solution.

With the solution gradients known, one can add nodes in areas of high solution gradient. New shape functions must be created for the newly added nodes, along with any nodes in their domain of influence. Another benefit of the FCM with adaptive node placement is that any node outside of the new domains of influence does not need 
to be recalculated. Thus, when a new solution is computed only the new nodes and their close neighbours must be reformulated and a complete remeshing is completely unnecessary.

While a complete analysis of adaptive node placement could be the subject of a thesis in its own right, I present here several examples to illustrate the method, its power, and some of its complications.

\subsubsection{Examples and tests}

I return again to the step index fiber, for which we have known solutions and a simple geometry. This fiber will be used as an example for adaptive node placement. The first step is creating a very coarse node mapping, in this case of only 224 nodes, shown in Fig. 86(a). A solution is found using the standard g-FCM giving an effective index for the fundamental mode of $n_{\text {eff }}=3.41326035$, an error of $4.89 \times 10^{-3} \%$, and field magnitude in Fig. 86(b).

The magnitude of the solution to the fundamental mode (or in principle any desired mode) is determined for each node, $i$, as

$$
H_{m a g} \|_{i}=\sqrt{H_{x}^{2}\left\|_{i}+H_{y}^{2}\right\|_{i}}
$$

The magnitude squared of the rate of change of the magnitude of the field is calculated as

$$
\left\|\left(\nabla H_{m a g} \|_{i}\right)\right\|^{2}=\left(\frac{\partial}{\partial x} H_{m a g} \|_{i}\right)^{2}+\left(\frac{\partial}{\partial y} H_{m a g} \|_{i}\right)^{2},
$$

which makes use of the previously calculated shape functions $\mathbf{N}_{\mathbf{x}}$ and $\mathbf{N}_{\mathbf{y}}$. With the rate of change for each point calculated, the nodes with the highest values are 


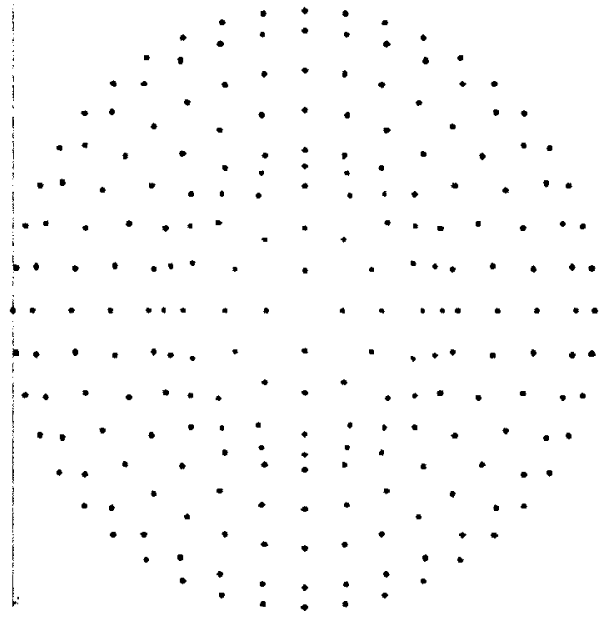

(a)

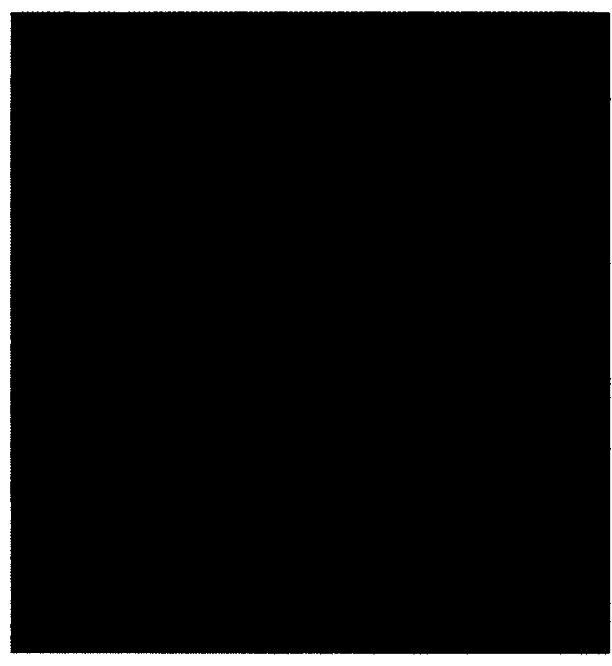

(b)

Figure 86: (a) A coarse node mapping of the step index fiber, with core nodes (yellow) and cladding nodes (blue). (b) field magnitude for the fundamental mode

selected. A histogram plot of the field rate of change for each point in the above coarse domain is shown in Fig. 87.

From this information, the points with the highest gradient are chosen and to these areas more points will be added. As an example, any point with a gradient greater than $40 \%$ of the maximum value will be used. This decision or cutoff point can vary quite a bit and would need to be optimized for a robust adaptive method. New points are now added in the surrounding region of the chosen high gradient points. This placement algorithm must also be studied and optimized.

The distribution of the new points must be close to the chosen points, however not too close as to create small groupings of points. The desire is to have a smoothly varying density of points. As such, the new points should extend a minimum of a third of a cloud size away from the chosen points to prevent grouping. Placing the new points even farther away may in fact be beneficial as this will extend the new nodes throughout the high gradient regions and slightly into the lower gradient 


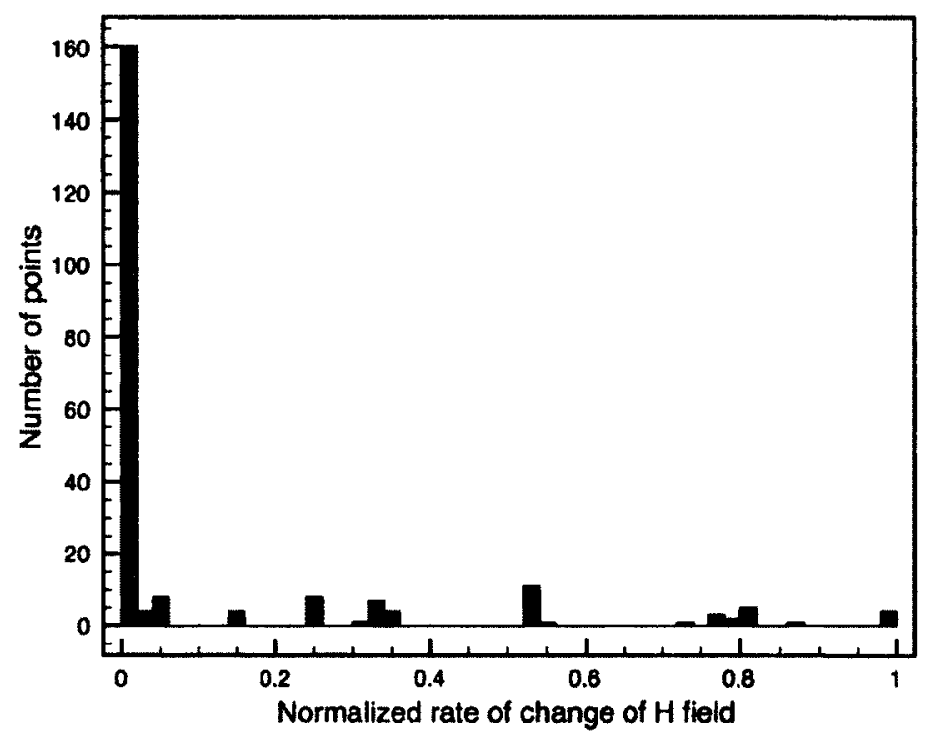

Figure 87: Histogram of the magnitude of the rate of change of the $H$ field for the points in the coarse step index fiber fundamental mode solution, values normalized to the maximum gradient.

regions creating a smooth transition.

The placement is also ideally done surrounding the node of interest. Initially, eight quadrants about the node, and off axis, are chosen in which to place the points. This ensures that each point gives optimal $d x$ and $d y$ information to the originating points, as described in [24]. The new points are at a radius of a fixed percent of cloud size and at angles $\pm 20^{\circ}, \pm 70^{\circ}, \pm 110^{\circ}$, and $\pm 160^{\circ}$. Choosing a radius of 0.7 times the cloud size we obtain the following point distribution after one iteration of adaptive node placement, Fig. 88.

These new nodes require both indices of refraction, along with the gradient of the index of refraction, if using the g-FCM. Adaptive placement has not been used in conjunction with the s-FCM as extra care must be used to place points on and near interface junctions, and the normals are required for the junctions. This creates an added layer of complexity and removes some of the elegance of the meshless methods 


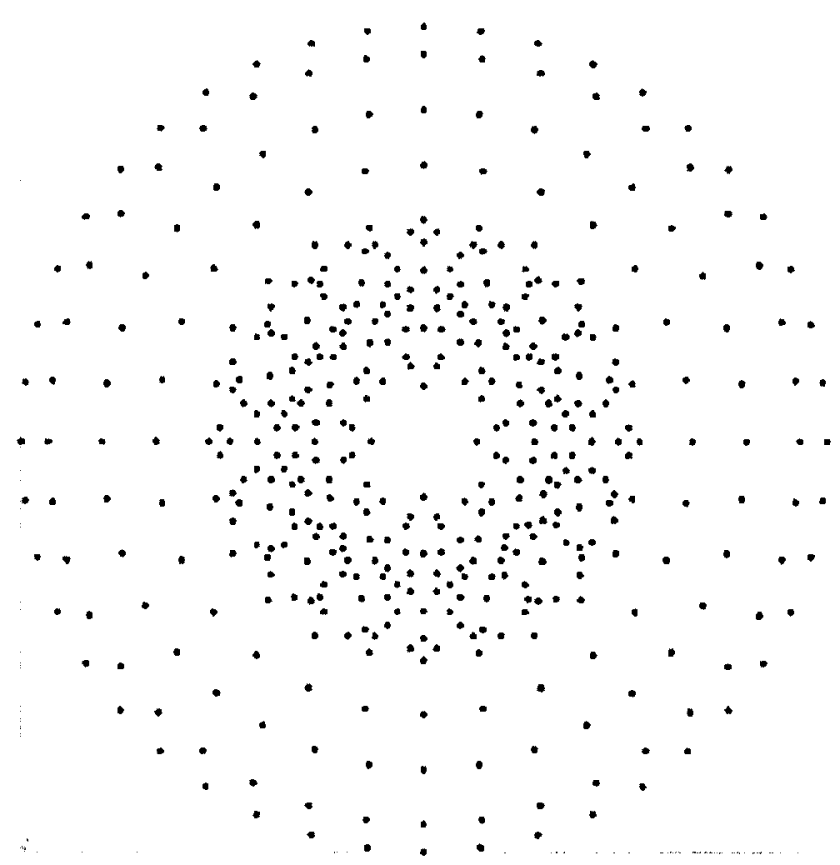

Figure 88: New node placement after one iteration of the adaptive refinement for the step-index fiber. New and cladding nodes (blue) original core nodes (yellow).

and adaptive refinement. Since the geometry of the materials is known, new points can be trivially assigned their index of refraction. The spatial derivatives will wait for the moment.

A list of new nodes along with all original points contained within their domain of influence is created, and new shape functions are created for only these points. These shape functions can then be multiplied with the known indices of refraction for the points to obtain spatial derivatives for the new nodes, giving all the required information for a new solution to the model.

The new model has 448 nodes, twice the original number of nodes, and gives an effective index for the fundamental mode of $n_{\text {eff }}=3.41311572$, an error of $6.54 \times$ $10^{-4} \%$. Thus with a doubling of points we have achieved an improvement in error of nearly a factor 10 . This has given an accuracy which for regular point spacing previously required roughly 3500 nodes. 


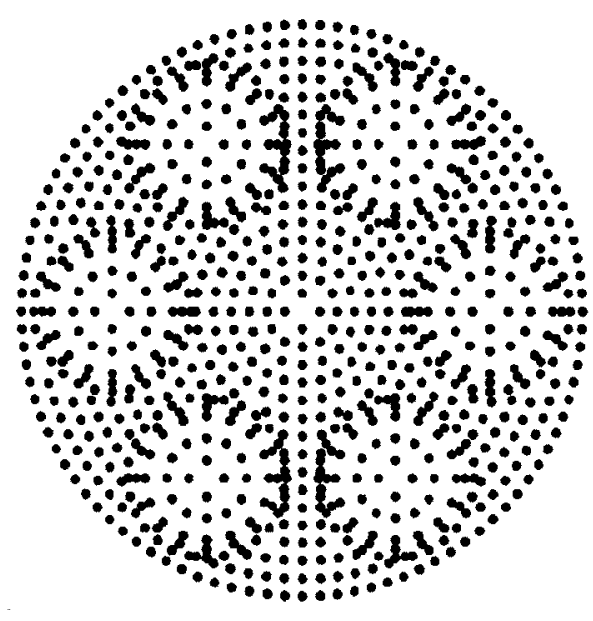

(a)

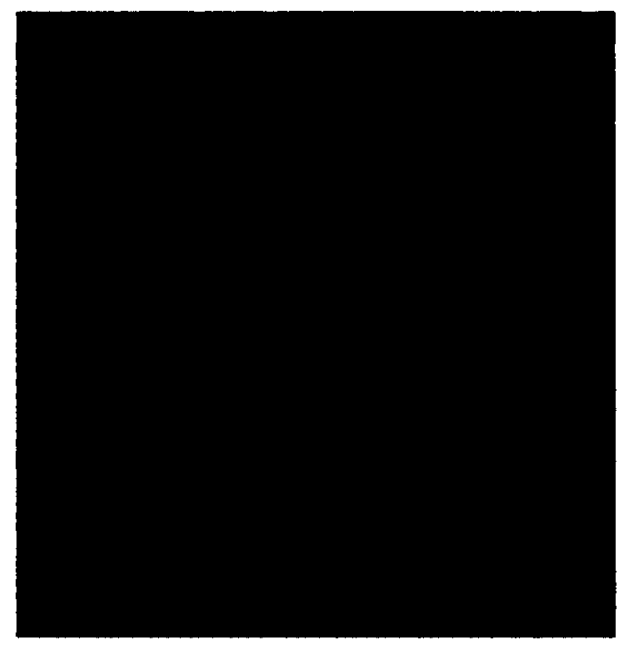

(b)

Figure 89: (a) A coarse node mapping of the air hole photonic crystal fiber, with photonic crystal nodes (red) and air holes nodes (blue). (b) field magnitude for the fundamental mode

This process has been applied to the holey fiber previously seen in Fig. 73(b). We begin with a coarse mesh having 874 points, Fig. 89 (a) and fundamental mode 89 (b). This gives an effective index of $n_{e f f}=1.4456180$ and an error off the previously published multipole method solution [65] of $1.54 \times 10^{-2} \%$. The following iteration increased the number of points to 1814 shown in Fig. 90. The new arrangement of nodes solves to a fundamental mode effective index of $n_{\text {eff }}=1.4456180$ and an error $1.07 \times 10^{-2} \%$, a somewhat improved result.

There are several complexities mentioned for this adaptive process. The first, which has yet to be mentioned, is that for each fiber there may be several modes of interest, each of which may have different regions of high field gradient. We have so far examined simply the first fundamental mode returned, which typically has the same regions of high gradient as the other corresponding fundamental mode. Higher order modes will have more regions which could just as easily been examined.

Identifying the regions of high gradient can also be examined. The points would 


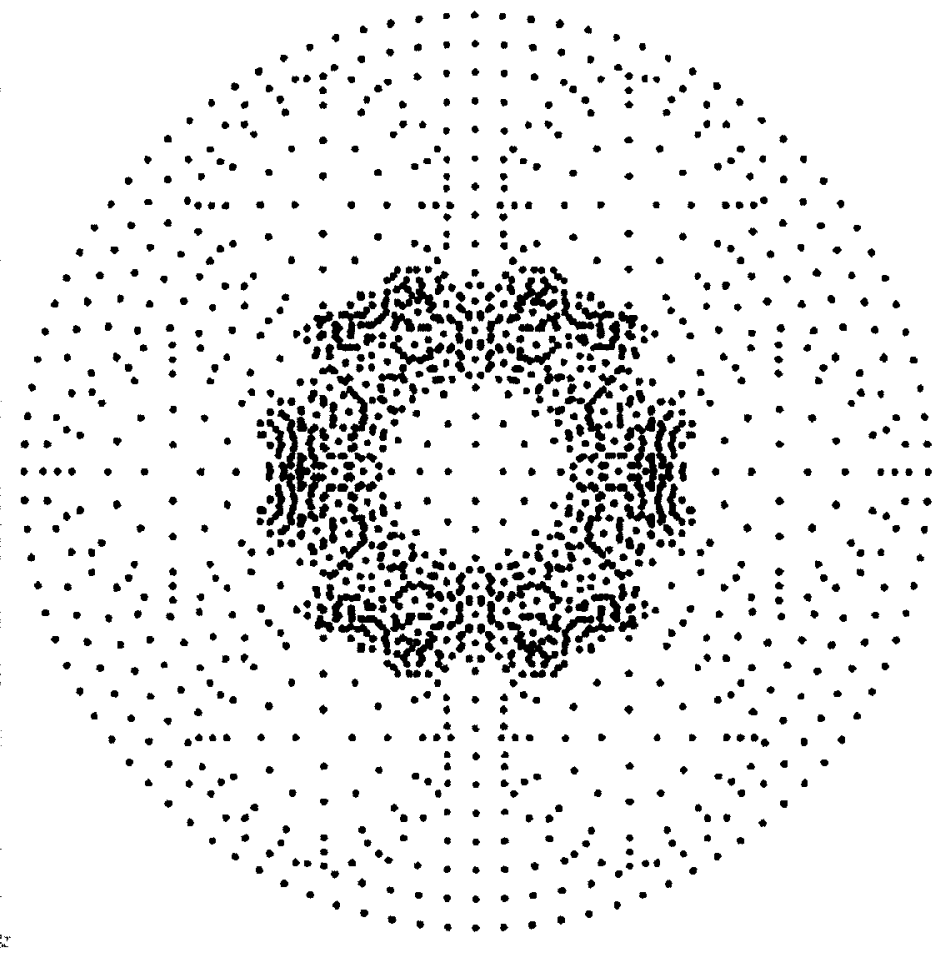

Figure 90: New node placement after one iteration of the adaptive refinement for the air hole photonic crystal fiber. New and photonic crystal nodes (red) air hole nodes (blue). 
ideally be weighted by their cloud size, as it is more important to add points to a region of high field gradient in a coarsely mapped area than a densely mapped area. A cutoff is also necessary to decide which nodal areas require more points. One could possibly weight these gradients adding more points to high gradients, fewer points to mid field gradients and none to low gradients. A balance must be used as one does not want to add too many new points for little added benefit, or too few points and require more iterations.

Placement of the new points is also a major consideration. If one knew the exact mode of interest and none of the others were desired, the nodes could be placed in the direction of the field gradient. This would add accuracy in precisely the direction

of field change. The spacing of the points is also a point of consideration. Placing a point too close to the original node creates disjointed groupings of high and low densities, too far, and it may begin to overlap with neighbouring nodes which may not be desired.

\subsection{Summary}

This chapter has introduced the problem of optical mode solving, a two dimensional vectorial eigenvalue problem. Optical mode solving is of interest as methods of fabricating microstructured waveguides are becoming more advanced and accurate modelling of their behaviour is needed.

Introduced, is a new method for treating inhomogeneous materials in PDEs, the graded index method or g-FCM. Both methods, the g-FCM and s-FCM, are implemented and used to solve for the optical modes of a variety of microstructured waveguides. 
Initial examples and tests use fully guided modes with Dirichlet boundary conditions. A step-index fiber example is first used and its fundamental eigenvalue modes are compared with known analytic solutions to confirm convergence FCM engine. A novel node placement technique is demonstrated, with a higher node density at the heterojunction interface, giving significant improvement to the solution accuracy.

Following the guided mode examples, a thorough study of two absorbing boundary conditions is conducted. Both transparent boundary conditions and a perfectly matched layer are used to solve leaky modes. Numerical implementation problems are discussed and significant improvement in the stability of the results is achieved. It was found that the transparent boundary conditions gave the most accurate answers without the need to fine tune any parameters.

Lastly, an attempt at adaptive node placement is presented which demonstrates the ease with which the technique can be performed using a meshless method. Improvements in accuracy are seen while using an intelligent node placement system based upon a previously solved system with a coarse node distribution. This technique could be further developed through improvements in the node distribution algorithms and by implementing it for the other equations studied in this thesis. Adaptive node placement represents a very interesting and potentially rewarding avenue for further research, its depth and scope being suitable for a research project in its own right. 


\section{Chapter 8}

\section{Conclusion}

A meshless solver for partial differential equations has been created based upon the finite cloud method. This method uses a collocation technique with a fixed reproducing kernel to approximate solutions to given PDE functions.

Several improvements have been made to the method to increase solution accuracy and stability, including nodal centering for consistency across the domains and cloud scaling which aids in the condition number of the moment matrix.

The solver has been expanded to solve materially inhomogeneous problems with increasing complication. Thermal analysis in steady state and transients has been thoroughly studied. Problems with mesa type structures have been identified and mechanisms to improve the solution have been found. An advanced conversion engine has been created and used to import advanced finite difference models from Atar and combine them with curvilinear models created specifically for the FCM. Model reduction techniques have been applied to large models including large opto-thermal models which have been spliced into several pieces and then stitched and solved at once. This has shown that the model reduction techniques are compatible with the FCM model representations and has been used to solve problems too large to solve on current hardware using standard techniques. 
We have adapted the FCM to solve hyperbolic wave equations, in particular steady state Schrodinger equations to solve for energy levels and wave functions, along with transients of a particle in a parabolic well. Initial tests of the method on fully vectorial 3D Maxwell equations have been performed although it is seen that the method does not necessarily lend itself to such analysis. That being said, this equation is quite complex and requires very large matrices and it is not a given that any analysis method is particularly adept at its solutions, although there are in fact many solvers designed to do so.

A thorough examination of optical mode solving has been performed using the FCM. The FCM has been used to accurately solve fully guided modes and provide an in depth analysis of absorbing boundary conditions in the solution of leaky modes. Several complex fibers have been solved and compared with both commercial solutions and analytical or previously published solutions. Node placement techniques and adaptive meshing have been implemented and tested for convergence showing good promise. Further work would place a greater emphasis on these methods in order to provide a robust solution. As well, model reduction technique should be studied more extensively and on a larger range of models and equation types. This powerful method has the ability to significantly aid in the solution of PDEs.

The FCM has proven quite adept at solving numerous problems of varying intricacies and complications. The ability to easily combine separate models without the need for remeshing or consistency checks have been shown to be quite advantageous. The ease with which adaptive meshing can be added to the models is also quite encouraging. Finally the ability to accurately model curvilinear models is a definite advantage over finite difference techniques which rely on rectilinear design layouts. 


\section{List of References}

[1] J. H. Davies. The Physics of Low-Dimensional Semiconductors: An Introduction. Cambridge University Press. ISBN 9780521484916 (1998).

[2] COMSOL Multiphysics. http://www. comsol.com. Version 4.1 Comsol Inc. (2011).

[3] A. Jain, I. Kostko, L. Chen, B. Xia, P. Dumais, and C. Callender. "Design and analysis of a 6-stage tunable mzi plc for bpsk generation." In "Circuits and Systems, 2007. MWSCAS 2007. 50th Midwest Symposium on," pages 1237 -1240. ISSN 1548-3746 (2007).

[4] P. Samadi, L. Chen, I. Kostko, P. Dumais, C. Callender, S. Jacob, and B. Shia. "Generating $4 \times 20 \mathrm{ghz}$ and $4 \times 40 \mathrm{ghz}$ pulse trains from a single 10-ghz modelocked laser using a tunable planar lightwave circuit." IEEE Photonics Technology Letters 22(5), 281 -282. ISSN 1041-1135 (2010).

[5] M. Renardy and R. Rogers. An introduction to partial differential equations. Texts in applied mathematics. Springer. ISBN 9780387004440 (2004).

[6] K. Singhal and J. Vlach. Computer Methods for Circuit Analysis and Design. Springer. ISBN 9781441947383 (2010).

[7] G. D. Smith. Numerical Solution of Partial Differential Equations. Oxford University Press (1974).

[8] S. D. T. Smy, D. Walkey. "A 3d thermal simulation tool for integrated devicesatar." Computer-Aided Design of Integrated Circuits and Systems 20, 105-115 (2001).

[9] T. R. Chandrupatla and A. D. Belegundu. Introduction To Finite Elements In Engineering. Prentice-Hall (1991). 
[10] D. H. Norrie and G. D. Vries. Introduction To Finite Element Analysis. Academic Press Inc. ISBN 0125216602 (1978).

[11] G. Liu. Meshfree Methods. CRC Press (2010).

[12] W. Hall. The Boundary Element Method. Kluwer Academic Publishers (1994).

[13] D. Poljak and C. A. Brebbia. Boundary Element methods for Electrical Engineers. WIT Press (2005).

[14] N. Aluru and G. Li. "Finite cloud method: a true meshless technique based on a fixed reproducing kernel approximation." Int. J. Numer. Meth. Engng 50, 2373-2410 (2001).

[15] S. Moslemi-Tabrizi. A Schrodinger solver for nano-scale one-particle QuantumWells, -Wires and -dots with infinite potential barriers. Dept. of Electrical Engineering, Concordia University, Montreal, Canada (2007).

[16] D. Burke, S. Moslemi-Tabrizi, and T. Smy. "Simulation of inhomogeneous models using the finite cloud method." Materialwissenschaft und Werkstofftechnik 41, 336-340 (2010).

[17] T. Smy and D. Burke. "Thermal models for optical circuit simulation using a finite cloud method and model reduction techniques." IEEE J. Technol. Computer Aided Des. (Accepted March 2013).

[18] D. R. Burke and T. J. Smy. "A meshless based solution to vectorial mode fields in optical microstructured waveguides." pages 82550L-82550L-10 (2012).

[19] D. R. Burke and T. J. Smy. "Optical mode solving for complex waveguides using a finite cloud method." Opt. Express 20(16), 17783-17796 (2012).

[20] D. R. Burke and T. Smy. "A meshless based solution to vectorial mode fields in optical micro-structured waveguides using leaky boundary conditions." $J$. Lightwave Technol. 31(8), 1191-1197 (2013).

[21] G. Liu and Y.T.Gu. An Introduction to Meshfree Methods and Their Programming. Springer (2005).

[22] R. Gingold and J. Monaghan. "Smoothed particle hydrodynamics - theory and application to non-spherical stars." Monthly Notices of the Royal Astronomical Society 181, 375-389 (1977). 
[23] R. Gingold and J. Monaghan. "Kernel estimates as a basis for general particle methods in hydrodynamics." Journal of Computational Physics 46(3), 429 453. ISSN 0021-9991 (1982).

[24] X. Jin, G. Li, and N. Aluru. "Positivity conditions in meshless collocation methods." Comput. Methods Appl. Mech. Engrg. 193, 1171-1202 (2004).

[25] G. Li, G. H. Paulino, and N. Aluru. "Coupling of the mesh-free finite cloud method with the boundary element method: a collocation approach." Computer Methods in Applied Mechanics and Engineering 192(2021), 2355 - 2375. ISSN 0045-7825 (2003).

[26] X. G. Wang. "A boundary value method for the singular behavior of bimaterial systems under inplane loading." International Journal of Solids and Structures 42(20), 5513 - 5535. ISSN 0020-7683 (2005).

[27] G. Li and N. Aluru. "Efficient mixed-domain analysis of electrostatic mems." Computer-Aided Design of Integrated Circuits and Systems, IEEE Transactions on 22(9), 1228 - 1242. ISSN 0278-0070 (2003).

[28] E. Cheney and D. Kincaid. Numerical Mathematics and Computing. Brooks/Cole. ISBN 9780495114758 (2007).

[29] G. Li and N. Aluru. "Efficient mixed-domain analysis of electrostatic mems." IEEE Transactions on Computer-Aided Design of Integrated Circuits and Systems 22(9), 1228-1242 (2003).

[30] J. Chan, G. Hendry, K. Bergman, and L. Carloni. "Physical-layer modeling and system-level design of chip-scale photonic interconnection networks." ComputerAided Design of Integrated Circuits and Systems, IEEE Transactions on 30(10), 1507 -1520. ISSN 0278-0070 (2011).

[31] T. Quarles, A. Newton, D. Pederson, and A. Sangiovanni-Vincentelli. SPICE 3 Version $3 F 5$ User's Manual. Dept. of EECE, Univ. of California, Berkeley.

[32] P. Gunupudi, T. Smy, J. Klein, and Z. J. Jakubczyk. "Self-consistent simulation of opto-electronic circuits using a modified nodal analysis formulation." IEEE Transactions on Advanced Packaging PP(99), 1 -15. ISSN 1521-3323 (2010).

[33] T. Smy, P. Gunupudi, S. McGarry, and W. N. Ye. "Circuit-level transient simulation of configurable ring resonators using physical models." J. Opt. Soc. Am. B 28(6), 1534-1543 (2011). 
[34] G. Li, X. Zheng, J. Yao, H. Thacker, I. Shubin, Y. Luo, K. Raj, J. Cunningham, and A. Krishnamoorthy. "High-efficiency $25 \mathrm{gb} / \mathrm{s}$ cmos ring modulator with integrated thermal tuning." In "Group IV Photonics (GFP), 2011 8th IEEE International Conference on," pages 8 -10. ISSN 1949-2081 (2011).

[35] J. E. Cunningham, I. Shubin, X. Zheng, T. Pinguet, A. Mekis, Y. Luo, H. Thacker, G. Li, J. Yao, K. Raj, and A. V. Krishnamoorthy. "Highly-efficient thermally-tuned resonant optical filters." Opt. Express 18(18), 19055-19063 (2010).

[36] J. Hofrichter, F. Horst, B. Offrein, O. Raz, T. de Vries, H. Dorren, P. Mechet, and G. Morthier. "Microdisc lasers coupled to silicon waveguides as versatile on-chip optical components for light generation, conversion and detection." In "Semiconductor Conference Dresden (SCD), 2011," pages 1 -4 (2011).

[37] S. J. Choi, K. Djordjev, S. J. Choi, and P. Dapkus. "Microdisk lasers vertically coupled to output waveguides." Photonics Technology Letters, IEEE 15(10), 1330 -1332. ISSN 1041-1135 (2003).

[38] R. Soref. "Silicon-based optoelectronics." Proceedings of the IEEE 81(12), 16871706. ISSN 0018-9219 (1993).

[39] R. Soref. "The past, present, and future of silicon photonics." IEEE Transactions on Selected Topics in Quantum Electronics 12(6), 1678-1687. ISSN 1077-260X (2006).

[40] Q. Xu, B. Schmidt, S. Pradhan, and M. Lipson. "Micrometre-scale silicon electrooptic modulator." Nature 435(7040), 325-327. ISSN 0028-0836 (2005).

[41] D. Celo, P. Gunupudi, R. Khazaka, D. Walkey, T. Smy, and M. Nakhla. "Fast simulation of steady-state temperature distributions in electronic components using multidimensional model reduction." IEEE Transactions on Components and Packaging Technologies 28(1), 70 - 79. ISSN 1521-3331 (2005).

[42] A. Odabasioglu, M. Celik, and L. Pileggi. "Prima: passive reduced-order interconnect macromodeling algorithm." Computer-Aided Design of Integrated Circuits and Systems, IEEE Transactions on 17(8), 645 -654. ISSN 0278-0070 (1998).

[43] J. W. Demmel and M. T. H. Y. "Applied numerical linear algebra." In "Society for Industrial and Applied Mathematics," SIAM (1997). 
[44] P. Gunupudi and M. Nakhla. "Multi-dimensional model reduction of vlsi interconnects." In "Custom Integrated Circuits Conference, 2000. CICC. Proceedings of the IEEE 2000," pages $499-502$ (2000).

[45] D. Celo, X. M. Guo, P. Gunupudi, R. Khazaka, D. Walkey, T. Smy, and M. Nakhla. "Hierarchical thermal analysis of large ic modules." IEEE Transactions on Components and Packaging Technologies 28(2), 207-217. ISSN 1521-3331 (2005).

[46] X. Guo, D. Celo, D. Walkey, and T. Smy. "A general method for the connection of a component thermal model to a board." Advanced Packaging, IEEE Transactions on 29(2), 250 - 263. ISSN 1521-3323 (2006).

[47] D. Celo, D. Walkey, and T. Smy. "Algorithmic approach for thermal port definition." Advanced Packaging, IEEE Transactions on 30(3), $491-498$. ISSN 1521-3323 (2007).

[48] MathWorks. http://www.mathworks.com/products/matlab/ (2011).

[49] R. Baets, P. Dumon, W. Bogaerts, A. Chelnokov, J.-M. Fedeli, L. Vanholme, and D. Steyaert. "Building technology platforms and foundries for photonic integrated circuits in europe." pages 69961D-69961D-6 (2008).

[50] D. Ding and D. Z. Pan. "Oil: a nano-photonics optical interconnect library for a new photonic networks-on-chip architecture." In "Proceedings of the 11th international workshop on System level interconnect prediction," SLIP '09, pages 11-18. ACM, New York, NY, USA. ISBN 978-1-60558-576-5 (2009).

[51] http://www.optiwave.com/products/spice_overview.html.

[52] D. M. Sullivan. Electromagnetic Simulation Using the FDTD Method. IEEE Press, Piscataway, NJ (2000).

[53] A. Taflove and S. C. Hagness. Computational Electrodynamics. Artech House, Inc., Norwood, MA (2005).

[54] A. Bondeson, T. Rylander, and P. Ingelström. Computational electromagnetics. Texts in applied mathematics. Springer. ISBN 9780387261584 (2005).

[55] Rsoft FemSim. http://www.rsoftdesign.com/products.php? sub= Component+Design\&itm=FemSIM. Version 3.3 Rsoft Inc. (2011). 
[56] P. Lusse, P. Stuwe, J. Schule, and H.-G. Unger. "Analysis of vectorial mode fields in optical waveguides by a new finite difference method." Joumal of lightwave technology 12, 487-494 (1994).

[57] J. D. Jackson. Classical Electrodynamics, volume 2009. Academic Press, New York. ISBN 0-471-30932-X (1998).

[58] R. B. Lehoucq, D. C. Sorensen, and C. Yang. "Arpack users guide: Solution of large scale eigenvalue problems by implicitly restarted arnoldi methods." (1997).

[59] GNU Octave. http://www.gnu.org/s/octave/ (2011).

[60] M. Panju. "Iterative methods for computing eigenvalues and eigenvectors." arXiv:1105.1185 (2011).

[61] K. Ramm, P. Lusse, and H.-G. Unger. "Multigrid eigenvalue solver for mode calculation of planar optical waveguides." IEEE Photonics Technology Letters 9(7), 967-969 (1997).

[62] P. McIsaac. "Symmetry-induced modal characteristics of uniform waveguides i: Summary of results." Microwave Theory and Techniques, IEEE Transactions on 23(5), 421 - 429. ISSN 0018-9480 (1975).

[63] J. M. Fini. "Improved symmetry analysis of many-moded microstructure optical fibers." J. Opt. Soc. Am. B 21(8), 1431-1436 (2004).

[64] G. Agrawal. Nonlinear Fiber Optics. Academic Press, 3 edition. ISBN 0120451433 (2001).

[65] H. Uranus and H. Hoekstra. "Modelling of microstructured waveguides using a finite-element-based vectorial mode solver with transparent boundary conditions." Optics Express 12(12), 2795-2809 (2004).

[66] Y.Y.Lu and J. Zhu. "Perfectly matched layer for acoustic waveguide modelling - benchmark calculations and perturbation analysis." Computer modelling in engineering and sciences 22(3), 235-247 (2007).

[67] C.-P. Yu and H.-C. Chang. "Yee-mesh-based finite difference eigenmode solver with pml absorbing boundary conditions for optical waveguides and photonic crystal fibers." Opt. Express 12(25), 6165-6177 (2004). 
[68] C.-H. Lai and H. chun Chang. "Effect of perfectly matched layer reflection coefficient on modal analysis of leaky waveguide modes." Opt. Express 19(2), 562-569 (2011). 